\title{
Ю.И. БЛОХ
}

\section{ТЕОРЕТИЧЕСКИЕ ОСНОВЫ КОМПЛЕКСНОЙ МАГНИТОРАЗВЕДКИ}

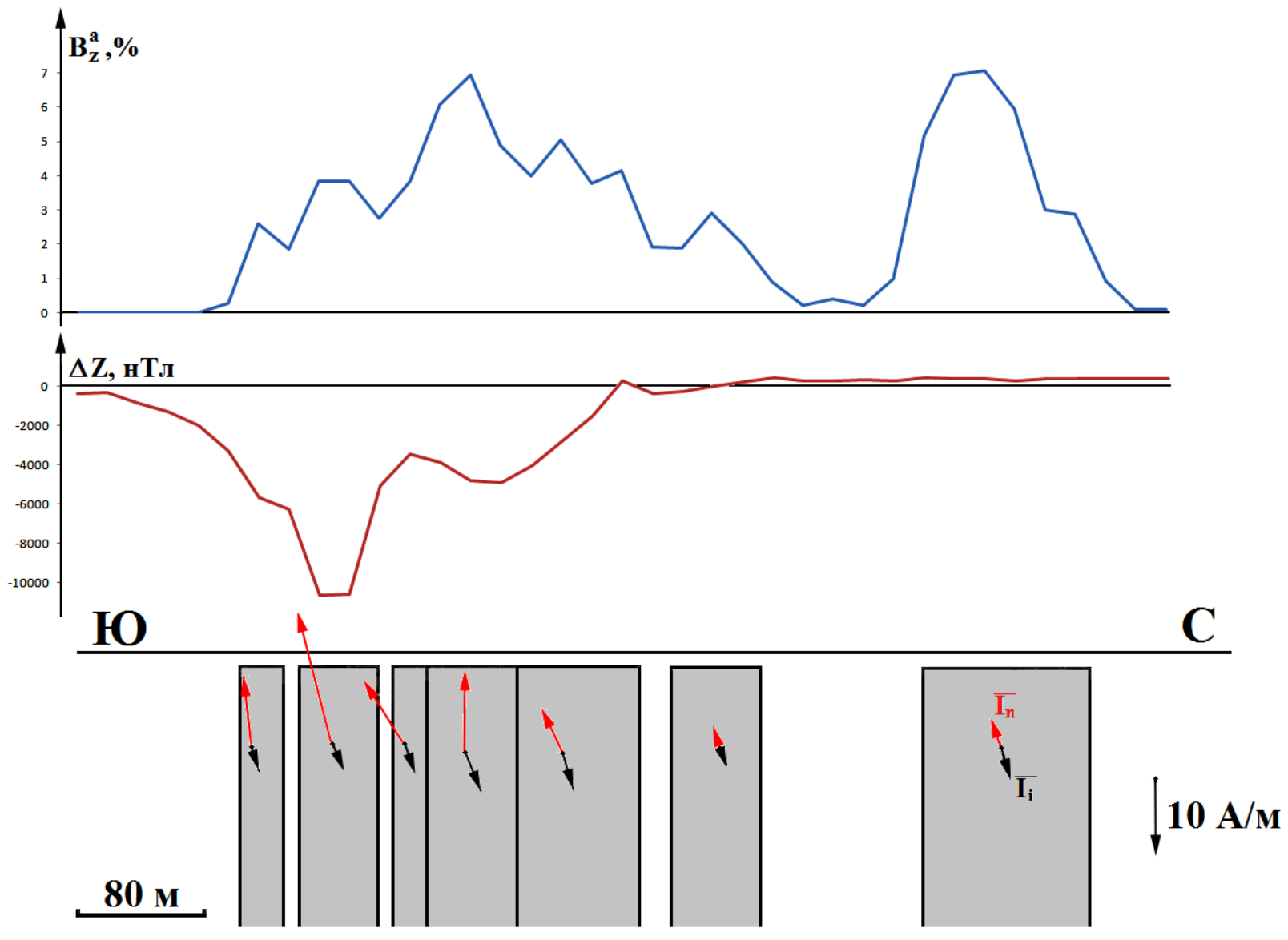

(С) Ю.И. Блох, 2012 


\section{ОГЛАВЛЕНИЕ}

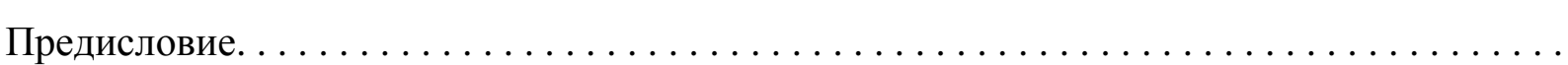

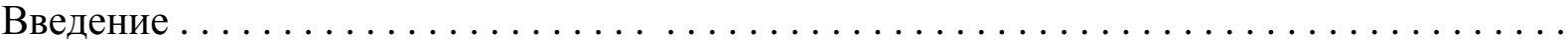

Глава 1. Физико-геологические основы комплексной магниторазведки. . . . . . . . . . . . .

$\S 1$. Магнитные свойства геологических объектов . . . . . . . . . . . . . . .

$\S 2$. Электромагнитные поля, изучаемые в комплексной магниторазведке. . . . . . . . .

2.1. Магнитное поле Земли. . . . . . . . . . . . . . . . . . . . . . . . . . . . . . . .

2.2. Магнитное поле постоянных токов. . . . . . . . . . . . . . .

2.3. Магнитное поле низкочастотных переменных токов . . . . . . . . . . . . . . . . . . . .

$\S 3$. Аномальные магнитные поля однородных шаров. . . . . . . . . . . . . . . . 22

Глава 2. Прямые задачи комплексной магниторазведки. . . . . . . . . . . . . 30

$\S 4$. Основные подходы к решению прямых задач. . . . . . . . . . . . . 30

$\S 5$. Объемное интегральное уравнение для намагниченности. . . . . . . . . . 34

$\S 6$. Численное решение интегральных уравнений для намагниченности. . . . . . . . 38

$\S$ 7. Процедура учета попарного взаимовлияния элементов. Экспресс-методика . . . 43

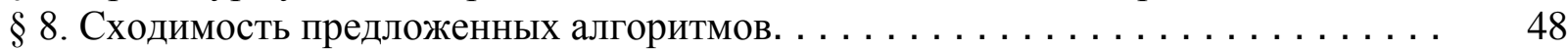

$\S$ 9. Оценка точности вычислений по предложенным алгоритмам. . . . . . . . . . . 50

Глава 3. Основные закономерности намагничения геологических объектов. . . . . . . . 56

$\S 10$. Намагничение многоугольных цилиндров и многогранников. . . . . . . . . 56

$\S 11$. Основные закономерности намагничения пластов в однородных полях . . . . . 62

$\S 12$. Особенности намагничения пластов в поле петли . . . . . . . . . . . . . 69

$\S 13$. Намагничение сближенных сильномагнитных объектов. . . . . . . . . . . 72

$\S 14$. Намагничение анизотропных складчато-разрывных структур . . . . . . . . 82

$\S 15$. Магнитные аномалии, возникающие в результате грозовых разрядов. . . . . . 85

$\S 16$. Влияние размагничивания на системы параметров источников, однозначно определяемые по аномальному полю. . . . . . . . . . . . . . .

Глава 4. Теоретические основы интерпретации данных комплексной магниторазведки . .

$\S$ 17. Возможности раздельного определения магнитной восприимчивости и остаточной намагниченности геологических объектов в рамках

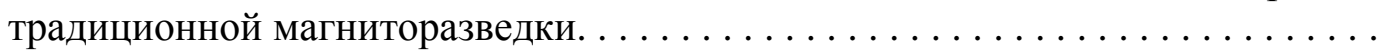

$\S 18$. Основы интерпретации данных магнитовариационного метода. . . . . . . . . 106

$\S 19$. Основы интерпретации данных многочастотных измерений в комплексной

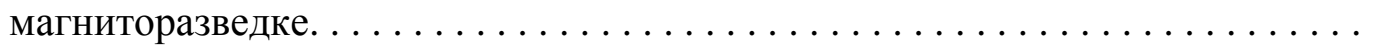

$\S 20$. Возможности многопетлевых измерений в комплексной магниторазведке . . .

$\S 21$. Модель эллиптического цилиндра в комплексной магниторазведке . . . . . . . .

$\S 22$. Модели крутопадающих пластов в комплексной магниторазведке. . . . . . . . .

$\S 23$. Основы комплексного моделирования магнитных геологических объектов. . .

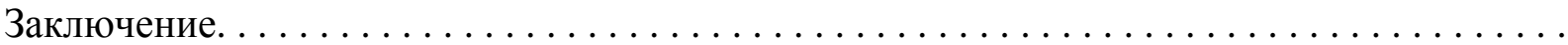

Приложение. Решение линейных обратных задач. $\ldots \ldots \ldots \ldots \ldots \ldots \ldots \ldots \ldots \ldots \ldots$

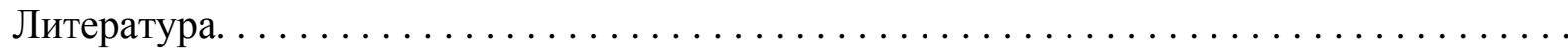




\section{ПРЕДИСЛОВИЕ}

«The time is out of joint: O cursed spite, That ever I was born to set it right!

Nay, come, let's go together».

W. Shakespeare. Hamlet

«Порвалась дней связующая нить.

Как мне обрывки их соединить!

Пойдемте вместе».

Перевод Б.Л. Пастернака

Приведенные в эпиграфе слова Гамлета как нельзя лучше формулируют замысел предлагаемой читателю работы. Комплексная магниторазведка, возникновение которой и практическое воплощение во многом были связаны с отечественной геофизической школой, в настоящее время находится почти на грани забвения. Многие ее создатели ушли из жизни, другие в трудных для геофизики условиях переключились на разработку иных ее направлений. В итоге сложилась ситуация, когда соединять обрывки связующей нити становится уже крайне сложно, тем не менее, автор попытался по мере сил осуществить это. Как у него получилось судить читателю.

Надо сказать, что, несмотря на свое название, предлагаемая работа ничуть не является плодом абстрактного теоретизирования. Авторское понимание теоретических возможностей комплексной магниторазведки сформировалось в значительной степени как раз в процессе решения практических задач. В течение 40 лет своей активной геофизической деятельности автор участвовал в полевых исследованиях с применением комплексной магниторазведки в таких регионах, как Урал, Кольский полуостров, КМА, Украина, Казахстан, Алтай и Горная Шория, Приангарье и т.д. Основное же время - более 15 лет - было уделено решению разнообразных геологических задач в Якутии: на многочисленных участках от Алданского до Анабарского щитов.

Автору посчастливилось быть лично знакомым со многими создателями комплексной магниторазведки, а с некоторыми из них встречаться не только на научных совещаниях, но и непосредственно в поле. Обо всех таких встречах в памяти остались самые теплые воспоминания, и всех хочется поблагодарить за удовольствие общения. Тем не менее, особое спасибо хочется сказать ближайшим коллегам: тем, с кем долгие годы совместно работали в Московском геологоразведочном институте (МГРИ) и который теперь именуется Российским государственным геологоразведочным университетом. С ними съеден не один пуд соли, и без них данная работа попросту не смогла бы появиться, так что, огромное спасибо Ирине Александровне Доброхотовой, Евгению Михайловичу Гаранскому, Игорю Владимировичу Ренарду, Сергею Зосимовичу Овешникову и многим сотрудничавшим с нами геофизикам. К сожалению, в 2011 году оборвалась жизнь многолетнего научного руководителя нашей группы Юрия Владимировича Якубовского, но благодарная память о нем хранится в наших сердцах.

Итак, попробуем собрать разрозненное воедино и создать стартовую модель для возрождения комплексной магниторазведки и ее дальнейшего развития.

Пойдемте вместе... 


\section{ВВЕДЕНИЕ}

Магнитные аномалии, изучаемые традиционным и одним из самых популярных методов разведочной геофизики - магниторазведкой - несут информацию о распределении индуктивной и естественной остаточной намагниченностей горных пород и руд. С ее помощью успешно решаются разнообразные геологические задачи, особенно, такие, которые не критичны к наличию точного знания природы намагниченности изучаемых объектов. Если же для правильного решения эти знания необходимы, традиционная магниторазведка обычно сталкивается с очень серьезными затруднениями. В качестве примера можно привести стандартную задачу классификации магнитных аномалий на «рудные» и «безрудные». Если не знать природу аномалии заранее, однозначно по данным традиционной магниторазведки решить вопрос, связана ли аномалия с индуктивно намагниченной рудой или же с безрудными породами с аномально высокой естественной остаточной намагниченностью, в общем случае невозможно. Преодолевать эти затруднения и предназначена комплексная магниторазведка.

Комплексная магниторазведка - это направление в геофизике, ориентированное на раздельное дистанционное изучение распределения магнитной восприимчивости и естественной остаточной намагниченности геологических объектов, другими словами, на раздельное изучение индуктивной и остаточной намагниченностей пород и руд в их естественном залегании (in situ).

В некоторых частных случаях раздельное изучение возможно и по данным традиционной магниторазведки путем анализа специфических проявлений эффекта размагничивания объектов в собственном аномальном поле. В общем же случае раздельного изучения можно добиться лишь путем комплексирования традиционной магниторазведки с другими геофизическими методами, данные которых определяются только индуктивной намагниченностью объектов и никак не связаны с их остаточной намагниченностью.

Вообще говоря, о том, что горные породы обладают индуктивной и остаточной намагниченностями, естествоиспытатели знают уже несколько веков. У.Паркинсон утверждает: «Гильберт заметил, что магнетит намагничивается в направлении магнитного поля Земли, а де Кастро в XVI веке обнаружил остаточную намагниченность горных пород» [142, c. 447]. Упомянутый первооткрыватель остаточной намагниченности горных пород Жуан де Каштру (João de Castro, 1500-1548), как правильнее именовать португальского полководца и исследователя, четвертого вице-короля португальской Ост-Индии, свою главную экспедицию осуществил в 1538-1541 гг. Тогда он, в частности, произвел на пути из Португалии в Индию несколько десятков наблюдений магнитного склонения. На основе данных, полученных им и рядом других исследователей, придворный врач английской королевы Елизаветы I - Уильям Гильберт (William Gilbert, 1544-1603) - подвел в 1600 г. первые итоги научного изучения геомагнитного поля в своем знаменитом трактате «О магните, магнитных телах и о большом магните - Земле», где, в частности, описал эксперименты с намагниченными шарами.

Систематическое изучение остаточной намагниченности горных пород началось в середине XIX века. Среди исследователей, внесших тогда наиболее весомый вклад в новое направление, следует отметить Жозефа Фурне (Joseph Jean Baptiste Xavier Fournet, 1801-1869), Ахилла Дилесса (Achille Joseph Delesse, 1817-1881), Македонио Меллони (Macedonio Melloni, 1798-1854) и Джузеппе Фольгерайтера (Giuseppe Folgheraiter, 1856-1913) [237]. После открытия закона Кюри (1895) и разработки теории парамагнетизма П. Ланжевеном (1910), а также теории ферромагнетизма П. Вейсом (1911) изучение магнитных свойств веществ становится одним из популярнейших научных направлений. К 30-м годам XX века магнетизм горных пород фактически выделился в самостоятельную отрасль науки [136], и с тех пор успешно развивается и совершенствуется. На его базе возникла палеомагнитология - учение о 
магнитном поле Земли прошлых геологических эпох [141].

К середине XIX века относится также возникновение магниторазведки. Один из ее основоположников - шведский физик Роберт Тален (Tobias Robert Thalén, 1827-1905) - пришел к мысли о том, что найти магнитную аномалию, связанную с рудами, недостаточно. Для поисков железных руд в то время достаточно успешно применялись различные варианты горных или рудничных компасов, в т. ч. так называемый «шведский горный компас». Это нехитрое приспособление было изобретено еще в XVIII веке горным советником Даниэлем Тиласом (1712-1772). Роберта Талена, в отличие от большинства современных ему исследователей магнитного поля, заинтересовал вопрос о том, нельзя ли помимо примерного определения местоположения руд, попытаться найти глубину их залегания и другие количественные характеристики. Такая постановка вопроса и привела к рождению магниторазведки, основной целью которой было объявлено определение параметров геологических объектов по создаваемым ими магнитным полям. Вот как Р. Тален описал суть предлагаемого метода в своей обобщающей работе 1877 г. «Исследование местностей на месторождения железных руд посредством магнитных измерений»: «...статья не имеет целью вытеснить рудничный компас из его употребления при отыскании железных руд, так как в этом отношении инструмент, без сомнения, весьма полезен. Напротив того, мы будем для сокращения этой статьи предполагать, что приблизительное положение рудных залежей уже определено, и что остается только узнать, в каком именно месте находится главное скопление рудной массы, на какой глубине лежит центр этой массы, какое притяжение имеет она и тому подобное...» [255].

С развитием магниторазведки ее неотъемлемой частью стало лабораторное изучение магнитных свойств образцов горных пород и руд, поскольку отсутствие информации о них зачастую выводит интерпретацию аномальных полей за пределы единственности искомого решения. Тем не менее, применительно к интерпретационным задачам, изучение магнитных свойств пород и, тем более, сильномагнитных руд на извлеченных из объектов образцах имеет ряд принципиальных недостатков. На это указывали многочисленные исследователи: М.А. Грабовский (1949, 1953 гг.), Д.Л. Фингер (1951 г.), А.Г. Калашников и С.П. Капица (1952, 1955 гг.), Ф. Стейси (1958, 1964 гг.), В.И. Андреев (1960 г.), Т. Нагата и Х. Киношита (1964 г.), 3.А. Крутиховская, В.Н. Завойский, С.М. Подолянко и Б.Я. Савенко (1964 г.), Н.А. Иванов и В.А. Шапиро (1965, 1967, 1969, 1970 гг.), Г.М. Авчян (1967 г.), В.М. Кириллов и В.П. Апарин (1972 г.), Н.Ф. Данукалов (1973 г.), С.С. Лапин (1976 г.) и другие.

Основные источники ошибок, по их мнению, сводятся к следующему:

1) магнитная восприимчивость извлеченного керна магнетитовых руд и других сильномагнитных образований отличается от истинной из-за изменения температуры, давления, a также в результате магнитного и механического воздействий бурового или другого породоразрушающего инструмента в процессе отбора образца;

2) естественная остаточная намагниченность сильномагнитных пород и руд содержит значительные вязкую и динамическую компоненты и сравнительно легко разрушается при механических нагрузках, применяемых для отделения образца от массива.

Добавим к этому, что при отборе образцов далеко не всегда имеются возможности соблюдения принципа рандомизации, из-за чего определяемые значения физических свойств могут оказаться непредставительными для изучаемых объектов. К тому же намагниченность образцов может значительно отличаться от истинной из-за существенных различий между размагничивающими полями в объекте и в извлеченном из него образце.

Все это побуждало к развитию комплексной магниторазведки, направленной на изучение магнитных свойств геологических объектов в естественном залегании и менее зависимой от их априорного знания, нежели традиционная магниторазведка.

Первым из дополняющих традиционную магниторазведку при дистанционном изучении магнитных свойств пород и руд стал магнитовариационный метод (МВМ). Его пытались применять для установления природы магнитных аномалий еще в XIX веке [143], а в начале XX века такие попытки стали повсеместными. Стоит отметить эксперименты одного из 
основоположников отечественной магниторазведки Э.Е. Лейста, который пытался оценивать изменения амплитуды магнитных вариаций на Курской магнитной аномалии (КМА) и на основании этого, в частности, предполагал ее связь с железными рудами [123]. Решающий же вклад в формирование данного метода в 1938 г. внес Б.М. Яновский [233].

Суть магнитовариационного метода состоит в следующем. При изменении магнитного поля Земли изменяется индуктивная намагниченность породы, а, следовательно, и величина магнитной аномалии. Таким образом, синхронно изучая вариации в базовой точке, находящейся в безаномальной зоне, и в перемещаемой по профилю текущей точке, можно выявить различия в протекании вариаций, а по ним получить информацию о магнитной восприимчивости породы.

Основным недостатком магнитовариационного метода, очевидно, являются помехи магнитотеллурического происхождения, также приводящие к различиям в протекании вариаций и усиливающиеся в зонах повышенной проводимости пород. Из-за них измерения приходится проводить длительное время, наблюдая солнечно-суточные и даже более медленные вариации, но и они оказываются не свободными от помех. Тем не менее, многие исследователи пытались совершенствовать магнитовариационный метод и применять его на практике. Среди них следует выделить Ю.В. Антонова, А.Г. Антонца, Я.Я. Биезайса, З.И. Борисову, В.А. Бугайло, С.К. Винокурова, М.В. Воробьева, Г.А. Гусманова, И.М. Евдокимова, Т.Я. Каменскую, В.М. Кириллова, В.В. Копаева, А.П. Корякина, М.И. Лапину, В.А. Ларионова, П.С. Мартышко, Ю.С. Набоко, В.А. Пьянкова, О.М. Распопова, С.В. Слюсарева, В.В. Стогния, Р.В. Терехову, В.Н. Туранова, Б.А. Ундзенкова, В.А. Филатова, В.Н. Филатову, М.И. Финкельштейна, С.Х. Хамидова, Н.Ф. Шевченко, Н. Голдштейна и С. Уорда.

Вторым методом, дополняющим традиционную магниторазведку при дистанционном изучении магнитных свойств пород и руд, стал метод искусственного подмагничивания (МИП), предложенный в 1957 г. двумя независимыми группами, возглавляемыми В.Д. Стадухиным и О.А. Соловьевым. В этом методе изучаемый объект индуктивно намагничивается искусственным источником, представляющим собой петлю достаточно больших размеров из изолированного провода, по которому пропускают сильный постоянный электрический ток. Индуцированное поле измеряется на поверхности Земли с помощью высокоточного магнитометра, и по его аномальной части делается вывод о величине магнитной восприимчивости.

Для учета первичного поля петли В.Д. Стадухин, приложивший наиболее заметные усилия к разработке МИП, предложил, в частности, применять следующий методический прием. Внутри основной квадратной двухвитковой петли вдоль профиля наблюдений и симметрично относительно него располагается прямоугольная компенсационная петля со специально рассчитанными размерами. Компенсационная петля подключается последовательно к основной петле так, чтобы создаваемые ими первичные поля в центре были противоположны по направлению и равны по модулю. Таким образом, в точке наблюдения суммарное поле источника равно нулю, а измеряемое магнитометром поле оказывается чисто аномальным. В такой модификации метод искусственного подмагничивания применяется при профилировании и зондировании, что дает возможность достаточно успешно решать упомянутые задачи классификации магнитных аномалий на «рудные» и «безрудные». Если необходимо проводить измерения в нескольких точках внутри петли на профиле, лежащем в плоскости ее симметрии и вытянутом вдоль компенсационного витка, то для учета первичного поля вводятся поправки. Существует также модификация с измерением аномального поля вне рамки.

В 1975 г. был проведен успешный эксперимент по применению для питания генераторной петли мощного МГД-генератора, который позволил создать с помощью квадратной рамки размерами $1 \times 1$ км подмагничивающее поле амплитудой около 50 нТл на расстоянии свыше 4 км от центра рамки [59]. К сожалению, развития этот эксперимент не получил.

Несмотря на трудоемкость и на отсутствие серийной аппаратуры для проведения измерений, метод искусственного подмагничивания получил распространение, преимущественно, в районах со спокойным рельефом. Методику МИП помимо В.Д. Стадухина 
и О.А. Соловьева совершенствовали Н.М. Бородаева, Р.Т. Васильев, В.В. Велинский, В.С. Вершинин, А.Я. Давыдов, А.Д. Дучков, Н.А. Ерошенко, Б.В. Жиромский, В.Ф. Захарченко, Т.А. Збыковская, В.М. Зыков, Н.А. Иванов, С.А. Казанцев, Л.И. Князев, А.Г. Кузьмин, А.Е. Медведев, А.Н. Молчанов, В.Н. Овчинников, И.Л. Ольшанецкая, Г.Г. Ремпель, В.В. Стогний, В.Н. Туранов, Г.Г. Улитина, Б.А. Ундзенков, Н.Н. Шабанова, Ю.В. Шумаков, А.А. Яковлев и другие исследователи. В теоретической проработке метода приняли активное участие Г.М. Воскобойников, Ю.М. Гуревич, М.М. Лаврентьев, А.И. Лучицкий, П.С. Мартышко, В.Н. Страхов, В.А. Филатов и А.В. Цирульский.

Наиболее широкое практическое распространение в комплексной магниторазведке среди методов, дополняющих традиционную магниторазведку при дистанционном изучении магнитных свойств пород и руд, получили низкочастотные индуктивные методы (НЧИМ). Первоначально они возникли как методы электроразведки хорошо проводящих руд, но затем были распространены и на магнитные объекты. В 1951 г. Джеймс Уэйт исследовал фундаментальную для НЧИМ задачу о шаре в однородном, гармонически меняющемся магнитном поле. Хотя основное внимание он уделил проводящим объектам, было упомянуто и о принципиальной возможности изучения магнитных тел. Исходя из данного решения, в 1959 г. Стэнли Уорд впервые проанализировал возможности применения низкочастотных индуктивных методов при изучении магнитных геологических объектов. Он показал, что глубину центра шара, его радиус, проводимость и магнитную восприимчивость можно однозначно определять на основании многочастотных измерений НЧИМ. В 1961 г. им была опубликована статья с результатами полевых экспериментов по применению НЧИМ в варианте дипольного индуктивного профилирования с треугольной вертикальной возбуждающей петлей на одном из магнетитовых месторождений Канады, но дальнейшего развития эти работы не получили. Сам же С. Уорд совместно с Н. Голдштейном на некоторое время заинтересовался магнитовариационным методом, но и эти работы вскоре прекратил. Судя по опубликованным данным, за границей соображения Уорда так и не были реализованы на производстве.

С 1965 г. в СССР были начаты систематические теоретические, опытно-методические и производственные работы по применению низкочастотных индуктивных методов для изучения магнитных свойств геологических объектов. Первые шаги в данном направлении были сделаны Ю.В. Якубовским и И.А. Доброхотовой в Московском геологоразведочном институте им. Серго Орджоникидзе (МГРИ). Они стали рассматривать НЧИМ не только как методы электроразведки, т.е. дистанционного изучения распределения электрических свойств горных пород и руд, но и как компонент комплексной магниторазведки, направленной на дистанционное изучение магнитных свойств. Полевые работы на месторождениях железистых кварцитов Кольского полуострова показали перспективность направления и обрисовали круг задач, которые необходимо было решить для успешного изучения магнитных объектов на разных стадиях геологоразведочных работ. Над решением этих задач помимо Ю.В. Якубовского и И.А. Доброхотовой стали работать Е.М. Гаранский, И.В. Ренард и автор. Результаты работ группы были подведены в 1986 г. выпуском монографии [45]. Разработки группы МГРИ были внедрены и успешно применялись в производственных масштабах в Южной Якутии, Казахстане, на Полярном Урале, в районе КМА, в Иркутской и Амурской областях при поисках и разведке месторождений магнетитовых руд. Опытные работы были проведены фактически во всех железорудных провинциях страны. Позже усилия группы сосредоточились на изучении среднемагнитных пород и руд, таких как гипербазиты, кимберлиты и породы трапповой формации, и продолжались вплоть до середины 90-х годов.

В НЧИМ при помощи стандартного и серийно выпускающегося оборудования производятся наблюдения индукции магнитной составляющей переменного гармонически меняющегося электромагнитного поля, создаваемого искусственными источниками. В качестве источников могут использоваться незаземленные петли различных конфигураций, питаемые низкочастотным переменным током от генератора, заземленные длинные кабели, магнитные диполи и их комбинации и т.п. Приемниками обычно служат магнитоиндукционные датчики или широкополосные индукционные преобразователи, э.д.с. которых измеряется с помощью 
микровольтметров. Высокая помехозащищенность аппаратуры достигается за счет фильтрации, чего нельзя добиться в методе искусственного подмагничивания на постоянном токе. Важнейшей особенностью НЧИМ является возможность проведения многочастотных измерений.

Аномалии, наблюдаемые с помощью низкочастотных индуктивных методов, по своей природе и по характеру частотной зависимости подразделяются, согласно Ю.В. Якубовскому, на четыре типа [231]. К первому относят вихревые аномалии, возникающие под действием вихревых токов, индуцированных в проводящих зонах разреза. Аномалии второго типа концентрационные - связаны с зарядами, индуцированными на поверхностях раздела сред с различной электропроводностью. Под влиянием поля этих зарядов происходит концентрация тока в областях с пониженным удельным электрическим сопротивлением. Третий тип поляризационные аномалии, аналогичные по своей природе аномалиям, изучаемым в методе вызванной поляризации. Наконец, четвертый тип аномалий, благодаря которому низкочастотные индуктивные методы применяются для изучения магнитных геологических объектов, - это магнитостатические аномалии, связанные с намагничиванием пород и руд в первичном поле источника. Эти аномалии несут информацию о магнитной восприимчивости разреза. В некоторой степени они близки к аномалиям, наблюдаемым при магниторазведочных исследованиях, но отличаются двумя существенными особенностями. Во-первых, они полностью свободны от влияния естественной остаточной намагниченности пород и руд. Вовторых, первичное поле, индуцирующее их, можно по желанию исследователя фокусировать в определенных частях разреза и выбирать его направление.

Аномалии всех типов взаимосвязаны, и соотношения между ними существенно зависят от характера первичного поля, а именно, от его ориентации, частоты, размеров источника и т.п. При изучении магнитных геологических объектов исследователь, проводя многочастотные измерения, имеет возможность убедиться, что наблюдаемые им аномалии принадлежат именно к магнитостатическому типу и что их можно интерпретировать по формулам магнитостатики.

Успехи группы МГРИ в изучении магнитных объектов стимулировали появление новых методик низкочастотных индуктивных исследований. Среди них следует в первую очередь отметить методику дипольного индуктивного профилирования. Г.С. Франтов, Г.Н. Новожилов и А.П. Савин применили ее при поисках железорудных месторождений [212], а В.Х. Захаров и А.В. Колесник - для опробования железистых кварцитов в карьерах в процессе эксплуатации [85].

В начале 70-х годов И.М. Евдокимов предложил применять для решения задач классификации магнитных аномалий т.н. метод индуктивности и взаимной индуктивности петель. В дальнейшем в его разработке и применении на магнетитовых и медноколчеданных месторождениях приняли участие Р.В. Терехова, Г.В. Селезнева и другие [81-82]. Суть метода состоит в изучении изменения индуктивности квадратной незаземленной петли, перемещаемой вдоль профиля, или взаимной индуктивности двух соосных петель с соотношением сторон 1:2. Поскольку индуктивность петли пропорциональна потоку магнитной индукции, проходящей через нее, отмеченные параметры несут информацию о магнитной восприимчивости среды. Питание петли переменным током низкой частоты и применение мостовой схемы измерения дают возможность изучать частотные характеристики и оценивать вклад проводимости среды. И.М. Евдокимову удалось отчасти справиться с проблемой помехозащищенности аппаратуры, но результаты ее применения для изучения магнитных объектов остались единичными.

Таким образом, геофизики предлагали достаточно разнообразные методы и методики комплексной магниторазведки, но, хотя их теоретические основания, вообще говоря, едины, ранее все они рассматривались порознь. В настоящей работе предпринята попытка единого и систематического описания их теоретических основ, что, по мнению автора, должно послужить расширению применения данных методов. 


\section{ГЛАВА 1. ФИЗИКО-ГЕОЛОГИЧЕСКИЕ ОСНОВЫ КОМПЛЕКСНОЙ МАГНИТОРАЗВЕДКИ}

\section{§ 1. Магнитные свойства геологических объектов}

Наиболее полными характеристиками магнитных свойств вещества являются кривая его намагничивания и петля гистерезиса, параметры которых, вообще говоря, могут существенно изменяться под влиянием множества причин [64]. Применительно к проблемам магниторазведки, т.е. для описания намагничивания горных пород и руд в относительно слабом земном магнитном поле, столь исчерпывающие характеристики обычно являются избыточными. В этих условиях, переходя к упрощенной, но достаточно адекватной с точки зрения практики модели, магниторазведчики обычно полагают породы и руды т.н. «идеальными ферромагнетиками». Это дает возможность обойтись при решении большинства решаемых задач всего двумя магнитными свойствами слагающих геологические объекты горных пород и руд: магнитной восприимчивостью и естественной остаточной намагниченностью.

Магнитная восприимчивость $\kappa$ характеризует реакцию вещества на приложенное магнитное поле. Для однородного и безграничного пространства данная реакция применительно к проблемам магниторазведки обычно полагается линейной и записывается следующим образом:

$$
\overline{\mathrm{I}}=\kappa \overline{\mathrm{H}},
$$

где $\overline{\mathrm{H}}$ - вектор напряженности приложенного (намагничивающего) магнитного поля, а $\overline{\mathrm{I}}-$ возникающий под его действием магнитный момент единицы объема вещества, называемый индуктивной (индуцированной) намагниченностью. В системе СИ магнитная восприимчивость безразмерна, а напряженность и намагниченность измеряются в A/м.

Естественная остаточная намагниченность горных пород и руд $\overline{\mathrm{I}}_{\mathrm{n}}$ является векторной характеристикой и в соответствии с определением Т. Нагаты представляет собой «всю измеряемую остаточную намагниченность, которой порода обладает in situ» [135, c. 158], то есть в естественном залегании. В соответствии с этим определением $\overline{\mathrm{I}}_{\mathrm{n}}$ является суммой намагниченностей разного происхождения. Петрофизики выделяют довольно много видов намагничивания, приводящих к появлению остаточной намагниченности, каждый из которых получил собственное наименование. По Б.М. Яновскому основными среди них являются следующие:

«кратковременное намагничивание при постоянной температуре называется мгновенным намагничиванием, при комнатной температуре - нормальным;

намагничивание в постоянном поле при действии переменного поля с амплитудным значением напряженности, убывающим от величины насыщения для данного ферромагнетика до нуля, носит название идеального намагничивания;

намагничивание в постоянном магнитном поле при уменьшении температуры от точки Кюри до некоторой температуры получило название термонамагничивания;

намагничивание в постоянном магнитном поле при уменьшении температуры в интервале между точкой Кюри и комнатной температурой называется парциальным термонамагничиванием;

намагничивание в постоянном магнитном поле при химических реакциях и перекристаллизации, которые сопровождаются изменением размера зерен ферромагнетика, называют химическим;

намагничивание в постоянном магнитном поле при наложении или изменении механических напряжений называют пьезонамагничиванием, при неоднократном изменении или переменных нагрузках в неизменном поле - динамическим;

осаждение в магнитном поле взвешенных в жидкости или газе частиц, имеющих собственный магнитный момент любого происхождения, сопровождается ориентационным 


\section{намагничиванием;}

возрастание намагниченности в постоянном поле с течением времени есть вязкое намагничивание, при одновременном увеличении температуры происходит термовязкое намагничивание;

наконец, изменение в постоянном магнитном поле температуры в окрестности изотропной точки, где константа кристаллографической магнитной анизотропии переходит через нуль, сопровождается переходным термонамагничиванием» [235].

Когда однородное безграничное пространство обладает естественной остаточной намагниченностью, формула (1.1) для модели «идеального ферромагнетика» приобретает следующий общий вид:

$$
\overline{\mathrm{I}}=\kappa \overline{\mathrm{H}}+\overline{\mathrm{I}}_{\mathrm{n}} \text {. }
$$

Магнитная восприимчивость пород и руд в системе СИ связана с относительной магнитной проницаемостью $\mu$ соотношением $\mu=1+\kappa$ (в системе СГС эта связь имеет вид $\mu=1+4 \pi \kappa$ ). Абсолютная магнитная проницаемость $\mu_{\mathrm{a}}$ (в системе СИ) характеризуется соотношением $\mu_{\mathrm{a}}=\mu_{0} \cdot \mu$, где $\mu_{0}=4 \pi \cdot 10^{-7}$ Гн/м - магнитная постоянная.

Рассмотрим основные характеристики магнитных свойств подробнее. Известно, что в ферромагнитных кристаллитах, входящих в состав горных пород и руд, имеются оси легкого намагничивания [229]. Таковыми могут служить кристаллографические оси или длинные оси вытянутых зерен. Всякое упорядочивание этих осей приводит к появлению магнитной текстуры породы и к зависимости намагниченности изометричных объектов от направления намагничивающего поля. Порода становится анизотропной, а возникающая таким образом анизотропия называется текстурной. Помимо нее горные породы могут обладать и слоистой анизотропией, возникающей при переслаивании магнитных и немагнитных прослоев. Чаще же эти два типа анизотропии сочетаются, например, в железистых кварцитах, при этом анизотропию, следуя Л.Е. Шолпо [229], называют текстурно-слоистой.

Отсюда следует, что в общем случае магнитная восприимчивость $\kappa$ в формулах (1.1) и (1.2) является тензором, и только в частности - для изотропных веществ - скаляром. Это относится и к магнитной проницаемости.

При рассмотрении вопросов интерпретации магнитных аномалий для анизотропных объектов наиболее удобно и естественно применение аппарата матричной алгебры. С этой точки зрения в правой прямоугольной декартовой системе координат $(\mathrm{x}, \mathrm{y}, \mathrm{z})$ с осью $\mathrm{z}$, направленной вниз, $\overline{\mathrm{I}}, \overline{\mathrm{H}}$ и $\overline{\mathrm{I}}_{\mathrm{n}}$ в формуле (1.2) надо рассматривать как векторы-столбцы с компонентами

$$
\overline{\mathrm{I}}=\left(\begin{array}{c}
\mathrm{I}_{\mathrm{x}} \\
\mathrm{I}_{\mathrm{y}} \\
\mathrm{I}_{\mathrm{z}}
\end{array}\right), \quad \overline{\mathrm{H}}=\left(\begin{array}{c}
\mathrm{H}_{\mathrm{x}} \\
\mathrm{H}_{\mathrm{y}} \\
\mathrm{H}_{\mathrm{z}}
\end{array}\right), \quad \overline{\mathrm{I}}_{\mathrm{n}}=\left(\begin{array}{c}
\mathrm{I}_{\mathrm{nx}} \\
\mathrm{I}_{\mathrm{ny}} \\
\mathrm{I}_{\mathrm{nz}}
\end{array}\right) .
$$

Тогда тензор магнитной восприимчивости к будет представлять собой матрицу следующего вида:

$$
\kappa=\left(\begin{array}{lll}
\kappa_{11} & \kappa_{12} & \kappa_{13} \\
\kappa_{21} & \kappa_{22} & \kappa_{23} \\
\kappa_{31} & \kappa_{32} & \kappa_{33}
\end{array}\right) .
$$

Многочисленные экспериментальные исследования, проведенные 3.А. Крутиховской, В.Н. Завойским и другими геофизиками $[117,118]$, показали, что тензор к пород и руд с полосчатой текстурой характеризуется обычно двумя главными компонентами: $\kappa_{t}$ по направлению полосчатости и $\kappa_{\mathrm{n}}$ - поперек полосчатости, причем $\kappa_{\mathrm{t}}>\kappa_{\mathrm{n}}$. Отношение

$$
\mathrm{A}_{\kappa}=\kappa_{\mathrm{t}} / \kappa_{\mathrm{n}}
$$

называется коэффициентом анизотропии магнитной восприимчивости. Некоторые исследователи, в т.ч. Л.Е. Шолпо, под этим термином понимают обратную величину [229], но мы в дальнейшем будем придерживаться именно этого обозначения. 
Ю.И. Блох

Если систему координат $\left(\mathrm{x}_{1}, \mathrm{x}_{2}, \mathrm{x}_{3}\right)$ подобрать так, чтобы ось $\mathrm{x}_{3}$ в данной точке была направлена по нормали к полосчатости (слоистости), то тензор к примет наиболее простой вид:

$$
\kappa=\left(\begin{array}{ccc}
\kappa_{\mathrm{t}} & 0 & 0 \\
0 & \kappa_{\mathrm{t}} & 0 \\
0 & 0 & \kappa_{\mathrm{n}}
\end{array}\right) .
$$

В произвольной системе координат компоненты зависят не только от $\kappa_{\mathrm{t}}$ и $\kappa_{\mathrm{n}}$, но и от направляющих косинусов нормали к полосчатости $\overline{\mathrm{n}}$. В матричной форме тензор магнитной восприимчивости можно представить следующим образом:

$$
\kappa=\kappa_{\mathrm{t}} \mathrm{E}-\left(\kappa_{\mathrm{t}}-\kappa_{\mathrm{n}}\right) \mathrm{K}_{\mathrm{n}},
$$

где Е - единичная матрица, то есть

$$
\mathrm{E}=\left(\begin{array}{lll}
1 & 0 & 0 \\
0 & 1 & 0 \\
0 & 0 & 1
\end{array}\right),
$$

a $\mathrm{K}_{\mathrm{n}}$ - симметричная матрица третьего порядка с элементами $\mathrm{k}_{\mathrm{ij}}$ :

$$
\mathrm{k}_{\mathrm{ij}}=\cos \left(\overline{\mathrm{n}}, \mathrm{x}_{\mathrm{i}}\right) \cos \left(\overline{\mathrm{n}}, \mathrm{x}_{\mathrm{j}}\right), \quad \mathrm{i}, \mathrm{j}=1,2,3,
$$

представляющими попарные произведения направляющих косинусов нормали к полосчатости. Для упрощения записи здесь принято: $\mathrm{x}_{1}=\mathrm{x}, \mathrm{x}_{2}=\mathrm{y}$ и $\mathrm{x}_{3}=\mathrm{z}$. В.Н. Завойский предложил запись аналогичной формулы через азимут падения D и угол падения J полосчатости (слоистости) в виде [84]:

$$
\begin{gathered}
\kappa_{\mathrm{ii}}=\kappa_{\mathrm{t}}-\Delta \kappa \alpha_{\mathrm{i}}^{2}, \\
\kappa_{\mathrm{ij}}=\kappa_{\mathrm{ji}}=-\Delta \kappa \alpha_{\mathrm{i}} \alpha_{\mathrm{j}}, \quad \mathrm{i} \neq \mathrm{j},
\end{gathered}
$$

где

$$
\begin{gathered}
\Delta \kappa=\kappa_{t}-\kappa_{n}, \\
\alpha_{1}=\cos D \sin J, \\
\alpha_{2}=-\sin D \sin J, \\
\alpha_{3}=\cos \mathrm{J} .
\end{gathered}
$$

В настоящей работе модель идеализированного анизотропного ферромагнетика является основной. Из нее как частный случай будут вытекать результаты для изотропных объектов. В наиболее общем случае тензор к пород и руд может являться более сложным и зависящим не от двух, а от трех главных компонент.

При рассмотрении двумерных задач в декартовой системе координат (x,z) с осью z, направленной вверх, структура соотношений (1.3)-(1.9) сохраняется. Векторы $\overline{\mathrm{I}}, \overline{\mathrm{H}}$ и $\overline{\mathrm{I}}_{n}$ принимают вид:

$$
\overline{\mathrm{I}}=\left(\begin{array}{c}
\mathrm{I}_{1} \\
\mathrm{I}_{2}
\end{array}\right), \quad \overline{\mathrm{H}}=\left(\begin{array}{c}
\mathrm{H}_{1} \\
\mathrm{H}_{2}
\end{array}\right), \quad \overline{\mathrm{I}}_{\mathrm{n}}=\left(\begin{array}{c}
\mathrm{I}_{\mathrm{n} 1} \\
\mathrm{I}_{\mathrm{n} 2}
\end{array}\right) .
$$

Тензор магнитной восприимчивости записывается следующим образом:

$$
\kappa=\left(\begin{array}{ll}
\kappa_{11} & \kappa_{12} \\
\kappa_{21} & \kappa_{22}
\end{array}\right),
$$

причем для него формула (1.7) остается справедливой, если принять для двумерного пространства

$$
\mathrm{E}=\left(\begin{array}{ll}
1 & 0 \\
0 & 1
\end{array}\right)
$$

а элементы матрицы $\mathrm{K}_{\mathrm{n}}$ :

$$
\mathrm{k}_{\mathrm{ij}}=\cos \left(\overline{\mathrm{n}}, \mathrm{x}_{\mathrm{i}}\right) \cos \left(\overline{\mathrm{n}}, \mathrm{x}_{\mathrm{j}}\right), \quad \mathrm{i}, \mathrm{j}=1,2 .
$$


Аналогия формул дает возможность упростить рассмотрение вопросов, связанных с двумерными моделями.

Информация о распределении магнитных свойств горных пород и руд, получаемая с помощью комплексной магниторазведки, принципиально важна при решении разнообразных геологических задач, причем магнитная восприимчивость несет важнейшую информацию о концентрации ферромагнитных минералов в породе или руде. Л.Е. Шолпо сформулировал это следующим образом: «...к является, по существу, лишь статистической мерой содержания в породах ферромагнитных минералов титаномагнетитового ряда, и ее изменения главным образом определяются вариациями этого содержания» [229, с. 46]. Если порода либо руда обладает анизотропией магнитной восприимчивости, это в ряде случаев дает возможность прослеживать условия образования и изменения горных пород, в частности анализировать испытанные геологическими объектами давления. Данные вопросы рассматривались многими геофизиками, и с достаточно подробным обзором полученных ими результатов можно познакомиться в монографии Л.Е. Шолпо [229].

Информацию о естественной остаточной намагниченности горных пород и руд, благодаря изложенному выше разнообразию причин, приводящих к ее появлению, гораздо сложнее применять для решения геологических задач. Изучая образцы, можно пытаться выявлять отдельные по происхождению составляющие вектора $\overline{\mathrm{I}}_{\mathrm{n}}$, но in situ подобное в общем случае пока невозможно. Тем не менее, кое-какая информация о происхождении остаточной намагниченности может быть получена непосредственно по данным комплексной магниторазведки.

Если удается установить, что вектор остаточной намагниченности является достаточно выдержанным по всему объему изучаемого объекта, это, скорее всего, свидетельствует о термоостаточной природе $\overline{\mathrm{I}}_{\mathrm{n}}$. B традиционной магниторазведке проявления подобного могут при коллинеарности векторов индуктивной и остаточной намагниченностей заключаться в изменении амплитуды наблюдаемой аномалии вплоть до появления над рудными телами интенсивных отрицательных магнитных аномалий. Соотношения между остаточной $\overline{\mathrm{I}}_{\mathrm{n}}$ и

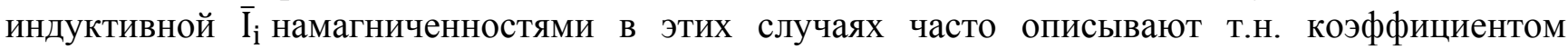
Кёнигсбергера Q, являющимся отношением их модулей:

$$
\mathrm{Q}=\frac{\left|\overline{\mathrm{I}}_{\mathrm{n}}\right|}{\left|\overline{\mathrm{I}}_{\mathrm{i}}\right|},
$$

хотя полной информации о направлении суммарного вектора намагниченности коэффициент Q, конечно не дает. Пусть, например, Q=1. Если направления векторов совпадают, это приводит к удвоению амплитуды магнитной аномалии, но, если направления, противоположны, магнитная аномалия над таким объектом практически пропадает. Последнее, кстати, встречалось на практике, в частности, над наиболее мощным магнетитовым рудным телом месторождения Кумдыколь в Казахстане [47] (см. рис. 69). Если векторы $\overline{\mathrm{I}}_{\mathrm{n}}$ и $\overline{\mathrm{I}}_{\mathrm{i}}$ не являются коллинеарными, коэффициент Кёнигсбергера становится еще менее удобным для анализа. При этом аномалии, наблюдаемые в традиционной магниторазведке, изменяют не только амплитуду, но и форму, затрудняя геологическое истолкование результатов. Комплексная магниторазведка выводит интерпретацию подобных аномалий на более высокий уровень.

Если же вектор остаточной намагниченности сильно варьирует по объему изучаемого объекта, это может быть связано с самыми разнообразными причинами. На неглубокозалегающих объектах источниками локальных изменений вектора $\overline{\mathrm{I}}_{\mathrm{n}}$, к сожалению, часто являются грозовые разряды (см. § 15). Продуцируемые ими магнитные аномалии никакого геологического значения, конечно, не имеют и являются с точки зрения геологии чистыми помехами. Помехи данного типа чрезвычайно затрудняют геологическое истолкование данных традиционной магниторазведки, и борьбу с ними, вообще говоря, можно эффективно вести лишь переходя к комплексной магниторазведке. 


\section{§ 2. Электромагнитные поля, изучаемые в комплексной магниторазведке}

\section{1. Магнитное поле Земли}

Комплексная магниторазведка представляет собой расширение традиционной магниторазведки, изучающей естественное магнитное поле Земли. Применительно к теории интерпретации, это поле зачастую безоговорочно рассматривается как статическое, хотя фактически это не вполне справедливо. На самом деле геомагнитное поле изменяется во времени, и эти изменения принято называть магнитными вариациями. Конечно, по сравнению с величиной постоянной части поля вариации сравнительно невелики, но для современной высокоточной аппаратуры достаточно заметны. В традиционной магниторазведке они рассматриваются, прежде всего, как помехи и устраняются в процессе съемки, в частности, с помощью вариационных станций. В комплексной же магниторазведке они по существу являются основой для применения магнитовариационного метода.

Основной характеристикой магнитного поля считается вектор его магнитной индукции $\overline{\mathrm{B}}$, который в системе СИ измеряется в Тесла. В долях этой единицы градуируются современные магнитометры. На поверхности Земли модуль индукции нормального геомагнитного поля изменяется примерно от 20 до 70 мкТл. Между индукцией $\overline{\mathrm{B}}$ и напряженностью $\overline{\mathrm{H}}$ магнитного поля существует следующее локальное соотношение: в произвольной точке пространства

где $\overline{\mathrm{I}}$ - намагниченность в рассматриваемой точке а.

$$
\overline{\mathrm{B}}(\mathrm{a})=\mu_{0}[\overline{\mathrm{H}}(\mathrm{a})+\overline{\mathrm{I}}(\mathrm{a})]
$$

Временные изменения геомагнитного поля весьма разнообразны по своему характеру и подразделяются на вековой ход, периодические вариации, короткопериодные колебания, бухтообразные возмущения и магнитные бури. Рассмотрим кратко их основные разновидности.

Вековым ходом (иначе вековыми вариациями) называют относительно медленные изменения величины и направления геомагнитного поля с периодами от десятков лет до нескольких тысячелетий. Среди наиболее изученных вариаций подобного типа выделяются 60летние с амплитудой до 500 нТл, которые прослеживаются на относительно локальных участках земной поверхности с площадями до нескольких тысяч км². Их природу связывают со строением нижней мантии. Внутри Земли находятся источники и других вековых вариаций.

Периодические вариации подразделяются по продолжительности своих характерных периодов на годовые, солнечно-суточные и лунно-суточные.

Годовые вариации имеют амплитуды от 10 до 25 нТл.

Солнечно-суточные вариации бывают двух видов: спокойные и возмущенные. Спокойные или невозмущенные солнечно-суточные вариации обозначают как $\mathrm{S}_{\mathrm{q}}$ от слов solar (солнечный) и quiet (спокойный). Они имеют амплитуду несколько десятков нТл и зависят преимущественно от широты пункта наблюдений и местного времени. Такая зависимость связана с тем, что их источниками являются электрические токи в ионосфере Земли на высоте $>100$ км. Изменяющиеся во времени магнитные поля этих токов индуцируют в Земле электрические токи. Возмущенные или иррегулярные солнечно-суточные вариации наблюдаются в дни магнитных бурь и обозначаются как $\mathrm{S}_{\mathrm{d}}$ от слова disturbed (возмущенный) их амплитуды могут на порядок превышать амплитуды $\mathrm{S}_{\mathrm{q}}$ вариаций.

Лунно-суточные вариации L имеют период 12,5 часов и амплитуду около 3 нТл.

Короткопериодные колебания (КПК) или геомагнитные пульсации представляют собой серии или цуги квазисинусоидальных колебаний. В зависимости от степени регулярности и периода КПК подразделяются на несколько типов. Те из них, у которых амплитуда постоянна или регулярно меняется, называются регулярными и обозначаются $\mathrm{P}_{\mathrm{c}}$ от слов pulsations continuous (регулярные пульсации) - их основные характеристики приведены в табл. 1. 
Таблица 1.

Параметры регулярных пульсаций.

\begin{tabular}{|c|c|c|}
\hline Обозначение & Период, с & Амплитуда \\
\hline $\mathrm{P}_{\mathrm{c} 1}$ & $0,2-5$ & $10-100$ пТл \\
\hline $\mathrm{P}_{\mathrm{c} 2}$ & $5-10$ & 100 пТл \\
\hline $\mathrm{P}_{\mathrm{c} 3}$ & $10-45$ & $1-2$ нТл \\
\hline $\mathrm{P}_{\mathrm{c} 4}$ & $45-150$ & 5 нТл \\
\hline $\mathrm{P}_{\mathrm{c} 5}$ & $150-600$ & $>10$ нТл \\
\hline $\mathrm{P}_{\mathrm{c} 6}$ & $>600$ & $10-100$ нТл \\
\hline \multicolumn{3}{|c}{} \\
\hline
\end{tabular}

Другие КПК представляют собой затухающие колебания, каждый цуг которых содержит от 5 до 20 пульсаций, а цуги следуют друг за другом через нерегулярные промежутки времени. Такие пульсации называют нерегулярными и обозначают как $\mathrm{P}_{\mathrm{i}}$ от слова irregular (нерегулярные) - их основные характеристики приведены в табл. 2.

Таблица 2.

Параметры нерегулярных пульсаций.

\begin{tabular}{|c|c|}
\hline Обозначение & Период, с \\
\hline $\mathrm{P}_{\mathrm{i} 1}$ & $1-40$ \\
\hline $\mathrm{P}_{\mathrm{i} 2}$ & $40-150$ \\
\hline $\mathrm{P}_{\mathrm{i} 3}$ & $>150$ \\
\hline
\end{tabular}

Бухтообразные возмущения выделяются на магнитограммах своеобразной формой графиков, напоминающих очертания морской бухты. Продолжительность подобного возмущения от 15-20 мин до 2-3 часов, амплитуда - от 30-40 до сотен нТл.

Наиболее интенсивные возмущения геомагнитного поля, охватывающие всю Землю и имеющие амплитуды до нескольких тысяч нТл, называются магнитными бурями. Они длятся до нескольких суток и происходят 1-2 раза в месяц. Гораздо чаще происходят магнитные суббури. Они, как правило, не имеют глобального характера и отмечаются наибольшими возмущениями в приполярных областях, точнее, в авроральных зонах.

Таким образом, применительно к решению геологических задач геомагнитное поле может рассматриваться как сумма постоянного и низкочастотного переменного полей, причем амплитуда переменной части поля намного меньше величины его постоянной части.

Теоретической базой анализа магнитного поля Земли, как и всех других электромагнитных полей, изучаемых в комплексной магниторазведке, является макроскопическая электродинамика, которая фактически состоит в решении уравнений Максвелла и в физической трактовке получаемых результатов [153].

Уравнения электродинамики устанавливают линейные дифференциальные связи между основными анализируемыми характеристиками, к которым помимо напряженности $\overline{\mathrm{H}}$ и индукции $\overline{\mathrm{B}}$ магнитного поля относятся также напряженность $\overline{\mathrm{E}}$ и индукция $\overline{\mathrm{D}}$ электрического поля, плотность тока проводимости $\overline{\mathrm{j}}$ и плотность свободных зарядов $\delta^{c в б}$. Эти уравнения обычно записывают следующим образом [5]:

$$
\begin{gathered}
\operatorname{rot} \overline{\mathrm{H}}=\overline{\mathrm{j}}+\frac{\partial \overline{\mathrm{D}}}{\partial \mathrm{t}}, \quad \operatorname{rot} \overline{\mathrm{E}}=-\frac{\partial \overline{\mathrm{B}}}{\partial \mathrm{t}}, \\
\operatorname{div} \overline{\mathrm{B}}=0, \quad \operatorname{div} \overline{\mathrm{D}}=\delta^{c \beta \sigma} .
\end{gathered}
$$

При учете магнитных вариаций приходится пользоваться полной системой уравнений, но для случая статического магнитного поля в отсутствие токов и свободных зарядов уравнения серьезно упрощаются и принимают следующий вид:

$$
\operatorname{rot} \overline{\mathrm{H}}=0, \operatorname{div} \overline{\mathrm{B}}=0 \text {. }
$$


На основании этих уравнений построена теория интерпретации данных традиционной магниторазведки.

\section{2. Магнитное поле постоянных токов}

В методе искусственного подмагничивания изучение распределения магнитной восприимчивости геологических объектов производится преимущественно в поле, создаваемом незаземленными петлями из изолированного провода, по которым пропускают постоянный электрический ток. В этом случае уравнения поля (2.2) принимают вид

$$
\operatorname{rot} \overline{\mathrm{H}}=\overline{\mathrm{j}}, \quad \operatorname{div} \overline{\mathrm{B}}=0 \text {. }
$$

Магнитные поля петель в непроводящей среде удобно и достаточно просто рассчитывать на основе известного закона Био-Савара-Лапласа [5], устанавливающего величину и направление вектора магнитной индукции $\mathrm{d} \overline{\mathrm{B}}$, которую создает элемент $\mathrm{d} \overline{\mathrm{l}}$ линейного тока $\mathrm{J}$ в среде с относительной магнитной проницаемостью $\mu=1+\kappa$ (см. рис. 1):

$$
\mathrm{d} \overline{\mathrm{B}}(\mathrm{a})=\frac{\mu_{0} \mu \mathrm{J}}{4 \pi} \frac{\left[\mathrm{d} \overline{\mathrm{l}} \times \overline{\mathrm{L}_{\mathrm{a}}}\right]}{\mathrm{L}_{\mathrm{qa}}^{3}} .
$$

Интегрируя (2.5) по контуру линейного тока l, получаем следующее выражение:

$$
\overline{\mathrm{B}}(\mathrm{a})=\frac{\mu_{0} \mu \mathrm{J}}{4 \pi} \oint_{1} \frac{\left[\mathrm{d} \overline{\mathrm{l}} \times \overline{\mathrm{L}_{\mathrm{qa}}}\right]}{\mathrm{L}_{\mathrm{qa}}^{3}} .
$$

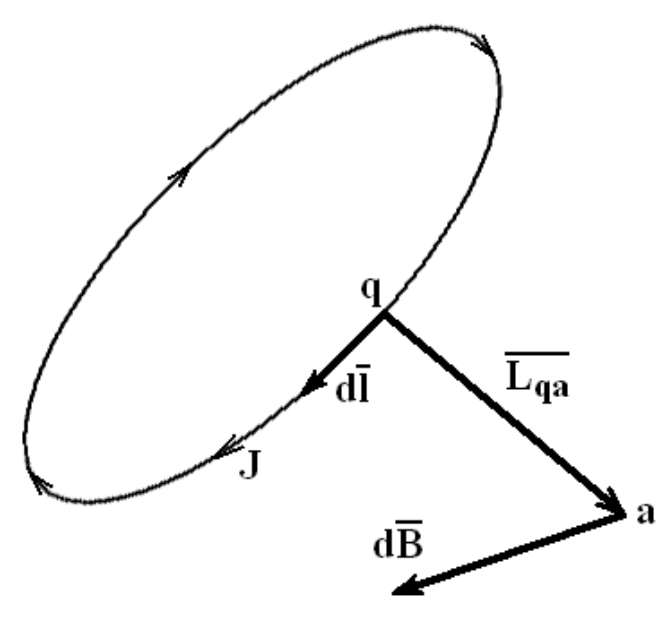

Рис. 1. Поле линейного тока

Для практических применений незаземленные петли обычно аппроксимируют набором прямоугольных отрезков с током. Тогда, интегрируя (2.6), легко получить формулы, пригодные для реализации на компьютерах. В немагнитной среде $\mu=1$, и формулы для компонент вектора магнитной индукции можно выразить в следующем - удобном для программирования - виде:

$$
\begin{aligned}
& B_{x}(x, y, z)=\frac{\mu_{0} J}{4 \pi} \sum_{n=1}^{N} B_{x n}\left(x, y, z, x_{n}, y_{n}, z_{n}, x_{n+1}, y_{n+1}, z_{n+1}\right), \\
& B_{y}(x, y, z)=\frac{\mu_{0} J}{4 \pi} \sum_{n=1}^{N} B_{y n}\left(x, y, z, x_{n}, y_{n}, z_{n}, x_{n+1}, y_{n+1}, z_{n+1}\right), \\
& B_{z}(x, y, z)=\frac{\mu_{0} J}{4 \pi} \sum_{n=1}^{N} B_{z n}\left(x, y, z, x_{n}, y_{n}, z_{n}, x_{n+1}, y_{n+1}, z_{n+1}\right),
\end{aligned}
$$

где для замкнутых петель ( $\mathrm{N}+1)$-ая точка совпадает с первой. Обозначим через $\mathrm{P}$ соотношение 
Ю.И. Блох

$$
\mathrm{P}=\frac{1}{|\overline{\mathrm{AB}}|^{2}|\overline{\mathrm{AC}}|^{2}-(\overline{\mathrm{AB}} \cdot \overline{\mathrm{AC}})^{2}}\left[\frac{(\overline{\mathrm{AB}} \cdot \overline{\mathrm{AC}})}{|\overline{\mathrm{AC}}|}-\frac{(\overline{\mathrm{AB}} \cdot \overline{\mathrm{BC}})}{|\overline{\mathrm{BC}}|}\right],
$$

где, в частности, $\overline{\mathrm{AB}}$ - вектор, направленный из точки $\mathrm{A}\left(\mathrm{x}_{\mathrm{n}}, \mathrm{y}_{\mathrm{n}}, \mathrm{z}_{\mathrm{n}}\right)$ в точку $\mathrm{B}\left(\mathrm{x}_{\mathrm{n}+1}, \mathrm{y}_{\mathrm{n}+1}, \mathrm{z}_{\mathrm{n}+1}\right)$; $\mathrm{C}(\mathrm{x}, \mathrm{y}, \mathrm{z})$ - точка, где вычисляется поле (рис. 2). Тогда

$$
\mathrm{B}_{\mathrm{xn}}=\mathrm{P}\left(\overline{1_{x}} \cdot[\overline{\mathrm{AB}} \times \overline{\mathrm{AC}}]\right), \quad \mathrm{B}_{\mathrm{yn}}=\mathrm{P}\left(\overline{1_{\mathrm{y}}} \cdot[\overline{\mathrm{AB}} \times \overline{\mathrm{AC}}]\right), \quad \mathrm{B}_{\mathrm{zn}}=\mathrm{P}\left(\overline{1_{z}} \cdot[\overline{\mathrm{AB}} \times \overline{\mathrm{AC}}]\right)
$$

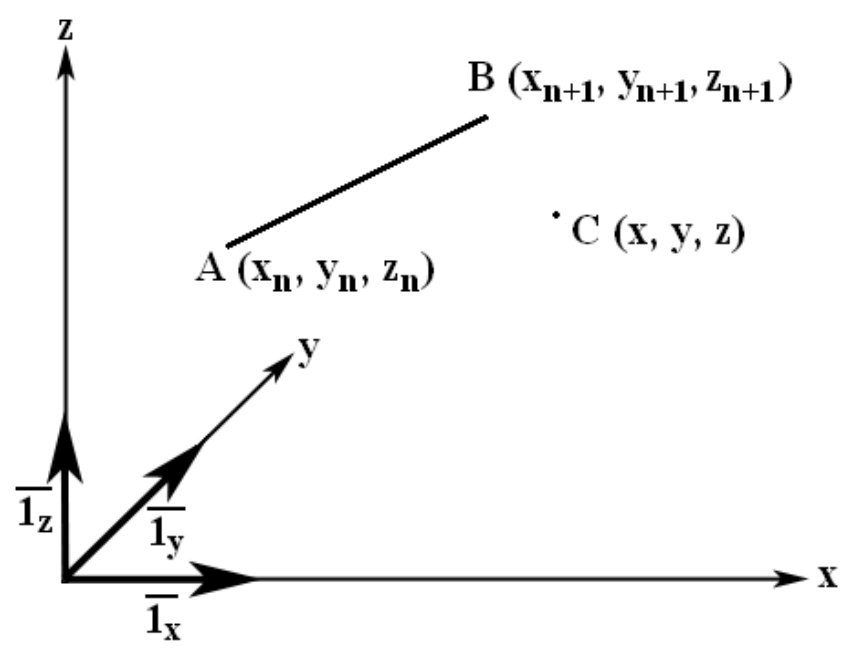

Рис. 2. К расчету поля прямолинейного отрезка проводника с током

Приведем подробную запись соотношений через координаты точек A, B и С:

$$
\begin{aligned}
& |\overline{\mathrm{AB}}|=\sqrt{\left(\mathrm{x}_{\mathrm{n}+1}-\mathrm{x}_{\mathrm{n}}\right)^{2}+\left(\mathrm{y}_{\mathrm{n}+1}-\mathrm{y}_{\mathrm{n}}\right)^{2}+\left(\mathrm{z}_{\mathrm{n}+1}-\mathrm{z}_{\mathrm{n}}\right)^{2}}, \\
& |\overline{\mathrm{AC}}|=\sqrt{\left(\mathrm{x}_{\mathrm{n}}-\mathrm{x}\right)^{2}+\left(\mathrm{y}_{\mathrm{n}}-\mathrm{y}\right)^{2}+\left(\mathrm{z}_{\mathrm{n}}-\mathrm{z}\right)^{2}}, \\
& |\overline{\mathrm{BC}}|=\sqrt{\left(\mathrm{x}_{\mathrm{n}+1}-\mathrm{x}\right)^{2}+\left(\mathrm{y}_{\mathrm{n}+1}-\mathrm{y}\right)^{2}+\left(\mathrm{z}_{\mathrm{n}+1}-\mathrm{z}\right)^{2}}, \\
& (\overline{A B} \cdot \overline{A C})=\left(x_{n+1}-x_{n}\right)\left(x-x_{n}\right)+\left(y_{n+1}-y_{n}\right)\left(y-y_{n}\right)+\left(z_{n+1}-z_{n}\right)\left(z-z_{n}\right), \\
& (\overline{\mathrm{AB}} \cdot \overline{\mathrm{BC}})=\left(\mathrm{x}_{\mathrm{n}+1}-\mathrm{x}_{\mathrm{n}}\right)\left(\mathrm{x}-\mathrm{x}_{\mathrm{n}+1}\right)+\left(\mathrm{y}_{\mathrm{n}+1}-\mathrm{y}_{\mathrm{n}}\right)\left(\mathrm{y}-\mathrm{y}_{\mathrm{n}+1}\right)+\left(\mathrm{z}_{\mathrm{n}+1}-\mathrm{z}_{\mathrm{n}}\right)\left(\mathrm{z}-\mathrm{z}_{\mathrm{n}+1}\right), \\
& \left(\overline{1_{x}} \cdot \overline{[A B} \times \overline{A C}\right]=\left(y_{n+1}-y_{n}\right)\left(z-z_{n}\right)-\left(z_{n+1}-z_{n}\right)\left(y-y_{n}\right), \\
& \left(\overline{1_{y}} \cdot \overline{[A B} \times \overline{A C}\right]=\left(z_{n+1}-z_{n}\right)\left(x-x_{n}\right)-\left(x_{n+1}-x_{n}\right)\left(z-z_{n}\right), \\
& \left(\overline{1_{z}} \cdot \overline{[\mathrm{AB}} \times \overline{\mathrm{AC}}\right]=\left(\mathrm{x}_{\mathrm{n}+1}-\mathrm{x}_{\mathrm{n}}\right)\left(\mathrm{y}-\mathrm{y}_{\mathrm{n}}\right)-\left(\mathrm{y}_{\mathrm{n}+1}-\mathrm{y}_{\mathrm{n}}\right)\left(\mathrm{x}-\mathrm{x}_{\mathrm{n}}\right) .
\end{aligned}
$$

Структура магнитного поля горизонтальной петли проиллюстрирована на рис. 3. Видно, что это поле, строго говоря, неоднородно, хотя вблизи центра петли на небольших глубинах изменяется не очень существенно.

Для получения более детальных представлений об этих изменениях обратимся к рис. 4, на котором поле петли изображено в виде изолиний его компонент. Рисунок свидетельствует, помимо прочего, о том, что для создания достаточного для прецизионных измерений подмагничивающего поля требуются весьма большие питающие токи. 
Ю.И. Блох

В методе искусственного подмагничивания, прежде всего в модификации магнитного зондирования, применяются также поля, создаваемые многопетлевыми источниками. Основой причиной их применения является стремление к такой структуре подмагничивающего поля, при которой в точке наблюдения на дневной поверхности это поле нулевое, а максимум его достигается на некоторой глубине. В.Д. Стадухин называл данный прием измерением «вертикальной составляющей вторичного магнитного поля в чистом виде» [170, с. 26].
Рис. 3. Векторы магнитного поля, создаваемого горизонтальной квадратной петлей, в вертикальной плоскости, проходящей через центр петли

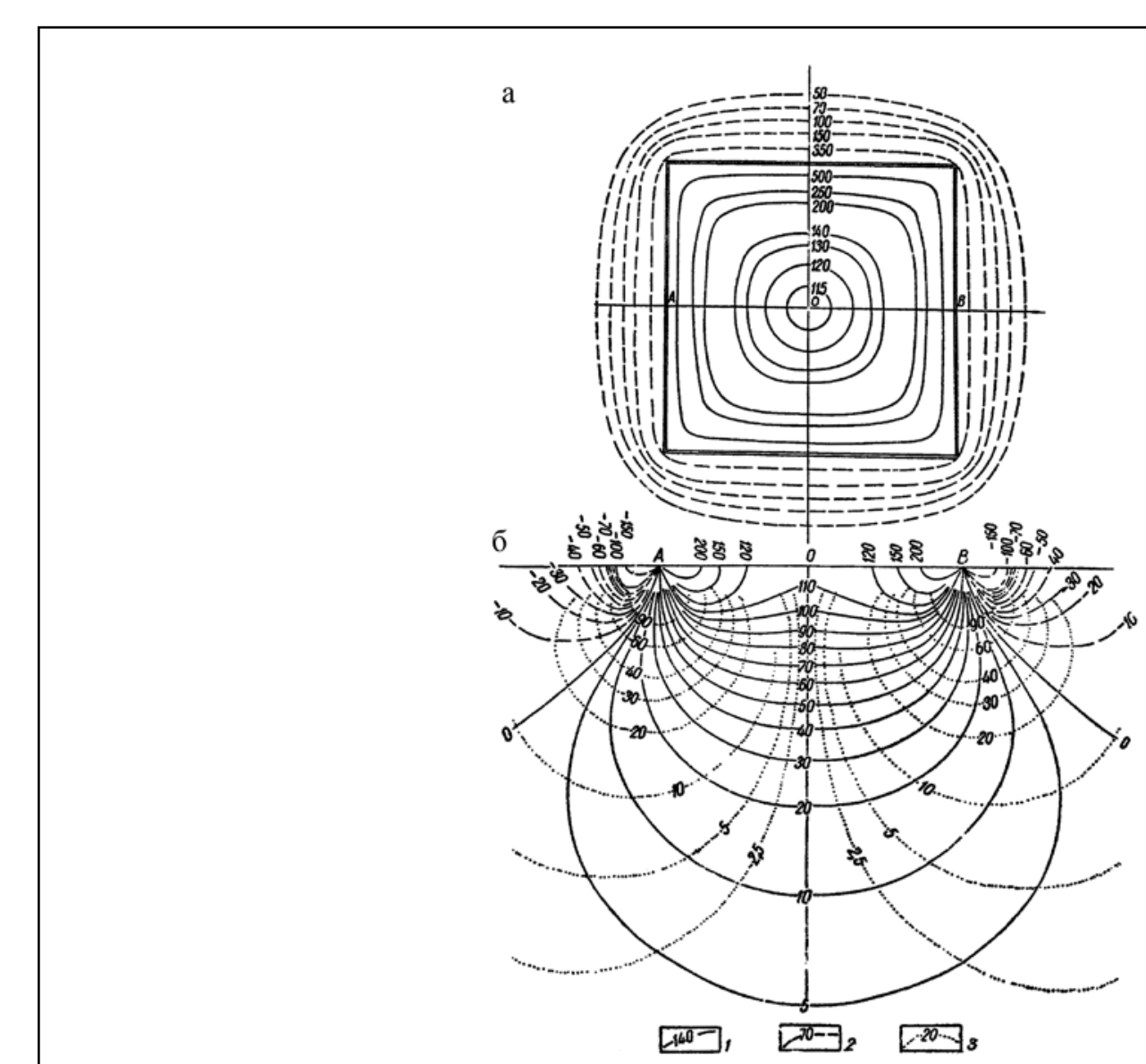

Рис. 4. Магнитное поле квадратной петли размерами $100 \times 100$ м, через которую пропущен постоянный ток 10 А: а) в плане, б) в разрезе через центральный профиль. Изодинамы в нТл:

1 - положительных значений вертикальной компоненты поля петли $\mathrm{B}_{z} ; 2$ - отрицательных значений $\mathrm{B}_{\mathrm{z}} ; 3$ - горизонтальной компоненты поля петли $\mathrm{B}_{\mathrm{x}}$ (по О.А. Соловьеву [156])

Чтобы достичь этого, используется дополнительная компенсационная петля. Суть компенсации состоит в том, что одновитковая прямоугольная петля размерами $2 \mathrm{R} \times 2 \mathrm{r}(\mathrm{R}>\mathrm{r})$ 
включается в цепь тока последовательно с двухвитковой квадратной намагничивающей петлей размерами $2 \mathrm{R} \times 2 \mathrm{R}$, но направления тока в них - противоположные (рис. 5a). Если длинная и короткая стороны компенсирующей прямоугольной петли связаны соотношением

$$
\mathrm{R}=\mathrm{r} \sqrt{7}
$$

то суммарное первичное поле постоянного тока в центре петель равно нулю. На рис. 5б показано, как изменяется первичное поле описанной установки под точкой наблюдения с изменением глубины. С удалением от дневной поверхности поле поначалу возрастает, достигает максимума на глубине $\mathrm{h}=0,45 \cdot \mathrm{R}$ и далее монотонно убывает. Помимо прочего, такая структура первичного поля помогает ослабить аномальный эффект от приповерхностных магнитных неоднородностей.

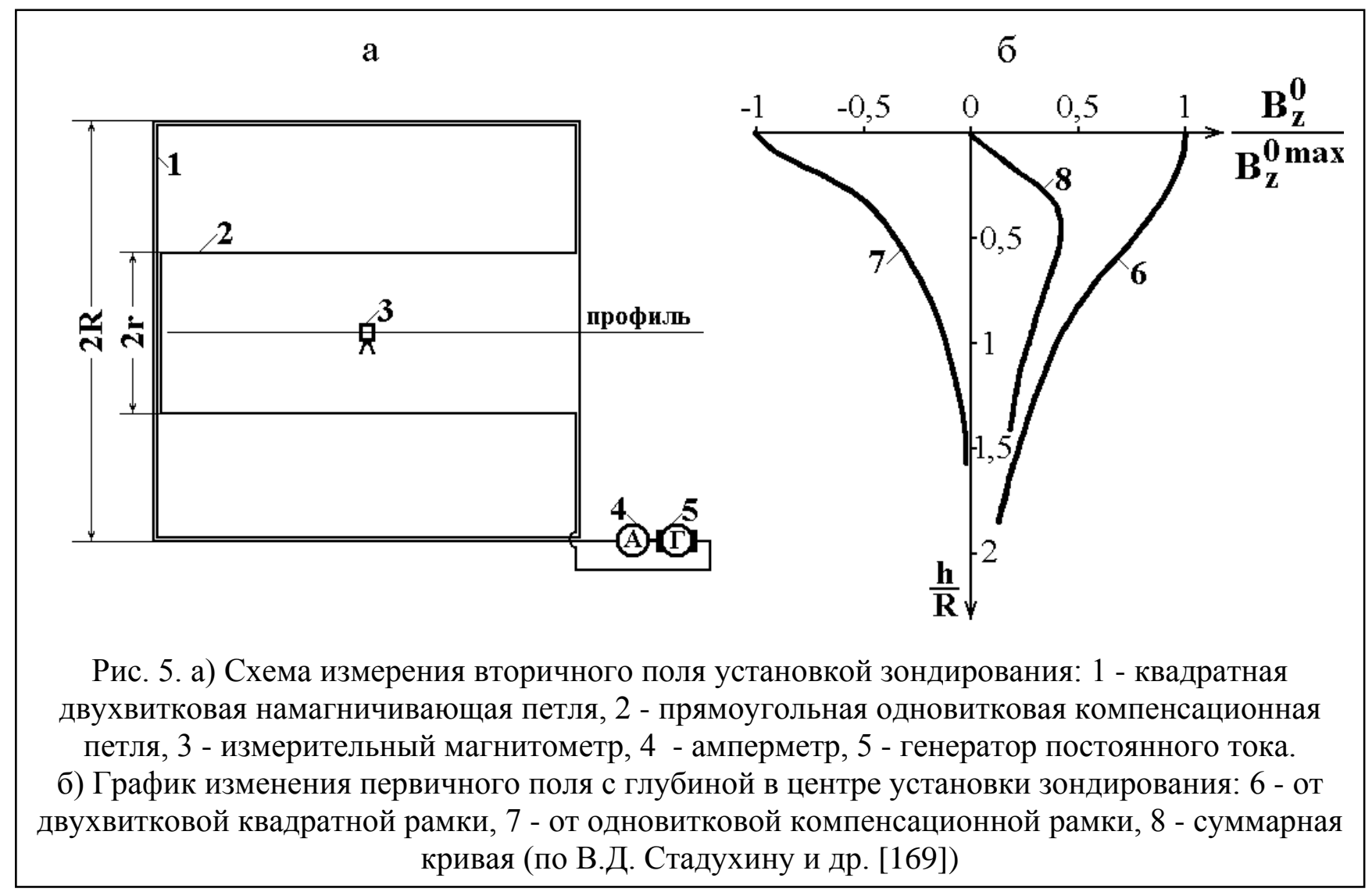

Очевидно, раскладка такого источника достаточно трудоемка и возможна с требуемым качеством лишь в районах, где нет дополнительных осложнений из-за рельефа местности. При современных возможностях топографического обеспечения геофизических работ реальную единовременную раскладку сложных петель на местности, вообще говоря, можно заменить последовательным выполнением съемок в разных прямоугольных петлях с последующим расчетом аномалий и вычислением линейных комбинаций этих аномалий. Тем самым можно получать фактические аномальные эффекты от сложных виртуальных источников с наперед заданными характеристиками распределения их первичного поля в пространстве, в частности, по глубине. Последнее означает принципиальную возможность послойного изучения геологической среды методами комплексной магниторазведки, что будет подробно рассмотрено в § 20. 


\section{3. Магнитное поле низкочастотных переменных токов}

Измерения в методе искусственного подмагничивания производятся не только на постоянном, но и на низкочастотном переменном токе. Рабочая частота некоторых реально применявшихся на практике индукционных преобразователей МИП составляла $0,7-1$ Гц [170, c. 16]. Низкочастотные индуктивные методы, как следует из самого их названия, вообще базируются на применении переменных токов, при этом рабочие частоты могут изменяться от первых герц до первых килогерц. Фактически наибольший объем проведенных ранее комплексных магниторазведочных исследований НЧИМ был выполнен с серийной электроразведочной аппаратурой типа ИКС и датчиками МИД-1 на рабочей частоте 22,5 Гц [45]. Все это значит, что в комплексной магниторазведке необходимо уделять пристальное внимание влиянию проводимости горных пород и руд на получаемые результаты.

Вообще говоря, учету проводимости нижнего полупространства при расчетах электромагнитных полей кабелей и петель посвящена обширная литература, с обзором которой можно познакомиться по книге А.Б. Великина и Г.С. Франтова [58]. С точки зрения теории наиболее простым представляется анализ поля круглой петли, через которую пропускается переменный ток. Рассмотрим вкратце решение этой задачи, полученное в 1965 г. Г.В. Присом [148].

Будем считать нижнее полупространство однородным и изотропным с удельной электропроводностью $\gamma$, относительной магнитной проницаемостью $\mu$ и относительной диэлектрической проницаемостью $\varepsilon$. На поверхности полупространства поместим круглую петлю радиуса а, обтекаемую переменным током $\mathrm{J}(\mathrm{t})=\mathrm{J} \cdot \mathrm{e}^{-\mathrm{i \omega t}}$. Здесь $\mathrm{J}$ - амплитуда силы тока, а $\omega$ - круговая частота, связанная с периодом $T$ соотношением $\omega=2 \pi / T$. Вообще говоря, распространение гармонического электромагнитного поля сопровождается изменением амплитуд колебаний и появлением фазовых сдвигов по отношению к фазе тока питания, принимаемой обычно за 0.

Исходными для решения задачи о поле петли над проводящим полупространством являются уравнения электродинамики (2.2), которые для гармонических полей можно записать в следующем виде [5]:

$$
\begin{gathered}
\operatorname{rot} \overline{\mathrm{H}}=\gamma \overline{\mathrm{E}}-\mathrm{i} \omega \varepsilon_{a} \overline{\mathrm{E}}+\overline{\mathrm{j}^{c m p}}, \quad \operatorname{rot} \overline{\mathrm{E}}=\mathrm{i} \omega \mu_{a} \overline{\mathrm{H}}, \\
\operatorname{div} \overline{\mathrm{B}}=0, \quad \operatorname{div} \overline{\mathrm{D}}=\delta^{c в \sigma},
\end{gathered}
$$

где $\overline{\mathrm{j}^{c m p}}$ - плотность стороннего тока, а $\mu_{a}$ и $\varepsilon_{a}$ - абсолютные проницаемости. Введем векторпотенциал $\overline{\mathrm{A}}$ соотношениями

где $\mathrm{k}$ - комплексное волновое число:

$$
\begin{gathered}
\bar{B}=\operatorname{rot} \bar{A}, \\
\bar{E}=i \omega\left(\bar{A}+\frac{1}{k^{2}} \operatorname{grad} \operatorname{div} \bar{A}\right),
\end{gathered}
$$

$$
\mathrm{k}^{2}=\omega^{2} \mu_{a} \varepsilon_{a}+\mathrm{i} \omega \mu_{a} \gamma
$$

Для низких частот, понятно, токами смещения можно пренебречь, и (2.13) для нижнего полупространства преобразуется к виду $\mathrm{k}^{2}=\mathrm{i} \omega \mu_{a} \gamma$. Для верхнего полупространства в воздухе $\gamma=0$, следовательно, там и $\mathrm{k}=0$.

Введем цилиндрическую систему координат $\mathrm{r}, \varphi, \mathrm{z}$ с осью $\mathrm{z}$, проходящей через центр петли. Вектор-потенциал первичного поля петли в воздухе имеет только одну компоненту $\mathrm{A}_{\varphi_{0}}$, равную

$$
A_{\varphi_{0}}=\frac{\mu_{0} J a}{2} \int_{0}^{\infty} J_{1}(\lambda a) J_{1}(\lambda r) e^{-\lambda z} d \lambda,
$$

где $\mathrm{J}_{1}$ - функция Бесселя. Применительно к рассматриваемой задаче уравнения (2.11) для вектор-потенциала (2.12) сводятся к волновому уравнению, которое достаточно просто решается методом разделения переменных с учетом соответствующих граничных условий [148]. Получаемый таким образом вектор-потенциал петли также обладает только одной 
компонентой $\mathrm{A}_{\varphi}$, которая для области низких частот при условиях $\left|\mathrm{k}^{2} \mathrm{a}^{2}\right| \ll 1$ и $\left|\mathrm{k}^{2} \mathrm{r}^{2}\right| \ll 1$ имеет следующий вид:

$$
A_{\varphi}=\frac{\mu_{0} J_{a}}{2} \int_{0}^{\infty} J_{1}(\lambda a) J_{1}(\lambda r) e^{-\lambda z}\left[\frac{\mu-1}{\mu+1}+\frac{\mu_{0} \mu}{\mu+1} \cdot \frac{k^{2}}{\lambda^{2}}\right] d \lambda .
$$

Напомним, что в данной формуле $\mu$ - относительная магнитная проницаемость нижнего полупространства, а $\mu_{0}=4 \pi \cdot 10^{-7} \Gamma \mathrm{H} / \mathrm{M}$.

Сводя интегральные выражения к эллиптическим интегралам I-го и II-го рода K(р) и Е(р), получаем для измеряемой обычно вертикальной составляющей поля петли $\mathrm{B}_{\mathrm{z}}$ [45]:

где

$$
\mathrm{B}_{\mathrm{z}}=\frac{1}{\mathrm{r}} \frac{\partial}{\partial \mathrm{r}} \mathrm{rA}_{\varphi}=\frac{\mu_{0} \mathrm{~J}}{2}\left[\mathrm{~B}^{*}(\mathrm{a}, \mathrm{r})+\frac{\mu-1}{\mu+1} \mathrm{~B}^{*}(\mathrm{a}, \mathrm{r})+\frac{\mu \mathrm{k}^{2} \mathrm{a}^{2}}{\mu_{0}(\mu+1)^{2}} \mathrm{~B}(\mathrm{a}, \mathrm{r})\right]
$$

$$
\begin{gathered}
\mathrm{B}^{*}(\mathrm{a}, \mathrm{r})=\frac{\mathrm{pa}}{2 \pi \sqrt{\mathrm{ar}}}\left[K(\mathrm{p})-\frac{1-(\mathrm{a}+\mathrm{r}) \mathrm{p}^{2} / 2 \mathrm{r}}{1-\mathrm{p}^{2}} \mathrm{E}(\mathrm{p})\right], \\
\mathrm{B}(\mathrm{a}, \mathrm{r})=-\frac{2}{\pi \mathrm{p}} \sqrt{\frac{\mathrm{r}}{\mathrm{a}}}[\mathrm{p} \sqrt{2} \mathrm{~K}(\mathrm{p})+\mathrm{E}(\mathrm{p})], \\
\mathrm{p}^{2}=\frac{4 \mathrm{ar}}{(\mathrm{a}+\mathrm{r})^{2}}, \\
\operatorname{Re} \mathrm{B}_{\mathrm{z}}=\frac{\mu_{0} J}{2 \mathrm{a}}\left[\mathrm{B}^{*}(\mathrm{a}, \mathrm{r})+\frac{\mu-1}{\mu+1} \mathrm{~B}^{*}(\mathrm{a}, \mathrm{r})\right], \\
\operatorname{Im} \mathrm{B}_{\mathrm{z}}=\frac{1}{\mathrm{r}} \frac{\partial}{\partial \mathrm{r}} \mathrm{rA}_{\varphi}=\frac{\mu \mathrm{k}^{2} \mathrm{a}^{2} \mathrm{~J}}{2 \mathrm{a}(\mu+1)^{2}} \mathrm{~B}(\mathrm{a}, \mathrm{r}) .
\end{gathered}
$$

Первое слагаемое в (2.20) представляет собой нормальное поле круглой петли в воздухе $\mathrm{B}_{\mathrm{z}}^{0}$, a второе - реакцию полупространства на возбуждение, другими словами, аномальное поле, которое обозначается как $\mathrm{B}_{\mathrm{z}}^{\mathrm{a}}$. $\mathrm{C}$ помощью полученных формул можно вычислять относительную магнитную проницаемость, либо магнитную восприимчивость нижнего полупространства.

Для практических применений необходимо иметь формулы, описывающие поле многоугольных петель. Наиболее детально проблема вычисления таких полей была исследована в работах А.В. Вешева и его сотрудников, которые опирались на полученное ранее В.А. Фоком и В.Р. Бурсианом решение задачи о поле прямолинейного и заземленного на концах кабеля [60-62]. Им удалось разработать весьма эффективный алгоритм расчета поля плоских многоугольных петель.

Очевидно, поле горизонтальной незаземленной петли, расположенной на проводящем полупространстве, представляет собой сумму полей составляющих ее прямоугольных кабелей, поэтому достаточно рассмотреть формулы для вычисления поля одного из таких кабелей. Для упрощения выражений расположим кабель длиной L вдоль оси Ox так, что его центр совпадает с началом координат. Вычисления удобно проводить в безразмерных прямоугольных координатах (x', y', z'), получаемых нормированием на половину длины кабеля:

$$
x^{\prime}=2 x / L, \quad y^{\prime}=2 y / L, \quad z^{\prime}=2 z / L .
$$

Введем также параметр $\delta$, связанный с волновым числом проводящего полупространства $\mathrm{k}$ и длиной кабеля следующим соотношением:

$$
\delta=\frac{|\mathrm{k}| \mathrm{L}}{2 \sqrt{2}}
$$

В системе СИ, выражая частоту питающего тока $\mathrm{f}$ в Гц и длину кабеля в м, параметр $\delta$ обычно вычисляют по формуле $\delta=0,99 \sqrt{\mathrm{f} / \rho}$. С точки зрения практики наиболее интересна область сравнительно небольших параметров $\delta \leq 4 \div 5$. В этом случае измеряемая чаще всего 
Ю.И. Блох

вертикальная компонента поля кабеля, как показано А.В. Вешевым и др. [60-62], на поверхности проводящего полупространства (при $\mathrm{z}=0$ ) может быть представлена в виде степенного ряда

$$
B_{z}\left(x^{\prime}, y^{\prime}, L\right)=\frac{\mu_{0} J}{\pi L} y^{\prime} \sum_{n=0}^{\infty}(-1)^{n+1} \frac{n^{2}-1}{(n+2) !}[\delta(1+i)]^{n} G_{n-3} .
$$

где

$$
\mathrm{G}_{\mathrm{n}-3}=\int_{-1}^{1}\left[\left(\xi^{\prime}-\mathrm{x}^{\prime}\right)^{2}+\mathrm{y}^{\prime 2}\right] \mathrm{d} \xi^{\prime} .
$$

Разделим (2.24) на действительную и мнимую части:

$$
\begin{gathered}
\operatorname{Re} B_{z}\left(x^{\prime}, y^{\prime}, L\right)=\frac{\mu_{0} J}{\pi L} y^{\prime}\left\{\frac{1}{2} G_{-3}-\frac{1}{7,5} \delta^{3} \cos \left(\frac{3 \pi}{4}\right)-\sum_{n=4,6,8, \ldots}^{\infty}\left[\delta^{n} \frac{n^{2}-1}{(n+2) !} G_{n-3} \cos \left(\frac{n \pi}{4}\right)-\right.\right. \\
\left.\left.-\delta^{n+1} \frac{(n+1)^{2}-1}{(n+3) !} G_{n-2} \cos \left(\frac{n+1}{4}\right)\right]\right\} ; \\
\operatorname{Im~} B_{z}\left(x^{\prime}, y^{\prime}, L\right)=\frac{\mu_{0} J}{\pi L} y^{\prime}\left\{-\frac{1}{8} \delta^{2} G_{-1}-\frac{1}{7,5} \delta^{3} \sin \left(\frac{3 \pi}{4}\right)-\sum_{n=4,6,8, \ldots .}^{\infty}\left[\delta^{n} \frac{n^{2}-1}{(n+2) !} G_{n-3} \sin \left(\frac{n \pi}{4}\right)-\right.\right. \\
\left.\left.-\delta^{n+1} \frac{(n+1)^{2}-1}{(n+3) !} G_{n-2} \sin \left(\frac{n+1}{4}\right)\right]\right\} .
\end{gathered}
$$

Амплитуда вертикальной компоненты поля петли $\mathrm{B}_{\mathrm{z}}$, естественно, вычисляется как

$$
\left|\mathrm{B}_{\mathrm{z}}\right|=\sqrt{\left.\left(\operatorname{Re} \mathrm{B}_{\mathrm{z}}\right)^{2}+(\operatorname{Im~B})_{\mathrm{z}}\right)^{2}}
$$

Вычисление функций $\mathrm{G}$ при этом производится по рекуррентным формулам:

$$
\begin{gathered}
G_{-3}=\frac{1}{y^{\prime}}\left(\operatorname{arctg} \frac{1-x^{\prime}}{\sqrt{\left(1-x^{\prime}\right)^{2}+y^{\prime 2}}}-\operatorname{arctg} \frac{1+x^{\prime}}{\sqrt{\left(1+x^{\prime}\right)^{2}+y^{\prime 2}}}\right) \\
G_{-1}=\ln \left(\frac{1-x^{\prime}+\sqrt{\left(1+x^{\prime}\right)^{2}+y^{\prime 2}}}{-1-x^{\prime}+\sqrt{\left(1-x^{\prime}\right)^{2}+y^{\prime 2}}}\right) \\
G_{n-3}=\frac{1}{n-2}\left\{(n-3) G_{n-5} y^{\prime 2}+\left(1-x^{\prime}\right)\left[\left(1-x^{\prime}\right)^{2}+y^{\prime 2}\right]^{\frac{n-3}{2}}+\left(1+x^{\prime}\right)\left[\left(1+x^{\prime}\right)^{2}+y^{\prime 2}\right]^{\frac{n-3}{2}}\right\} \\
G_{n-2}=\sum_{k=0}^{\frac{n}{2}-1}\left\{\frac{y^{\prime 2}\left[\left(1-x^{\prime}\right)^{n-2 k-1}-\left(-1-x^{\prime}\right)^{n-2 k-1}\right]}{n-2 k-1} \cdot \frac{\left(\frac{n}{2}-1\right) !}{k !\left(\frac{n}{2}-1-k\right) !}\right\} .
\end{gathered}
$$




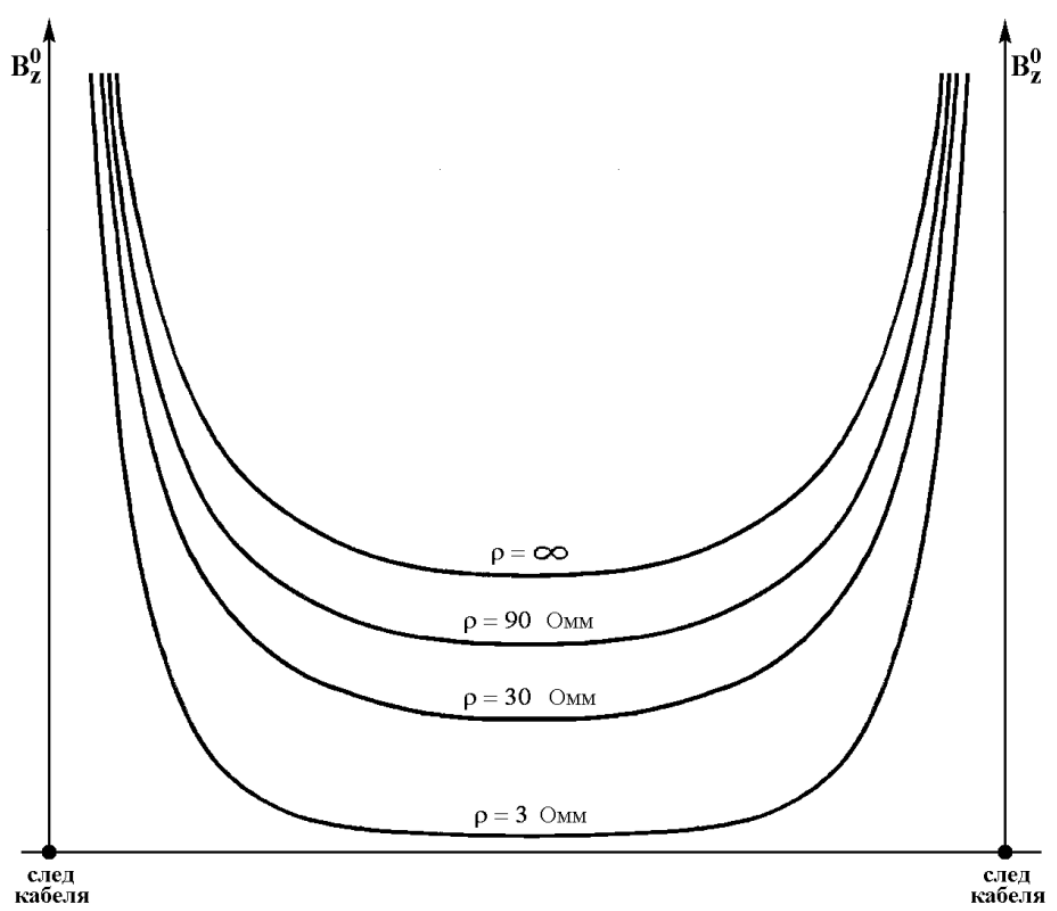

Рис. 6. Графики вертикальной компоненты нормального поля квадратной петли размерами $1 \times 1$ км на центральном профиле при разных значениях удельного сопротивления нижнего полупространства (по А.В. Вешеву и др. [62])

На рис. 6 показаны графики вертикальной компоненты нормального поля квадратной петли размерами $1 \times 1$ км на центральном профиле при разных значениях удельного сопротивления нижнего полупространства. Рисунок демонстрирует, что проводимость подстилающего полупространства приводит к частичному поглощению поля усиливающемуся с увеличением проводимости (или уменьшением сопротивления) и разному в разных частях профиля, что, естественно, требует учета при выделении аномальной составляющей [45].

В низкочастотных индуктивных методах аномалии $\mathrm{B}_{\mathrm{z}}^{\mathrm{a}}$ вычисляются по измеренным значениям $\mathrm{B}_{\mathrm{z}}$ в долях нормального поля в данной точке $\mathrm{B}_{\mathrm{z}}^{0}$ по формуле

и обычно выражаются в \%.

$$
\mathrm{B}_{\mathrm{z}}^{\mathrm{a}}=\frac{\mathrm{B}_{\mathrm{z}}-\mathrm{B}_{\mathrm{z}}^{0}}{\mathrm{~B}_{\mathrm{z}}^{0}}
$$

\section{§ 3. Аномальные магнитные поля однородных шаров}

Как следует из приведенного в предыдущих параграфах обзора, первичные поля, поляризующие природные объекты и изучающиеся в комплексной магниторазведке, обладают следующими общими характеристиками:

1. все рассматриваемые поля, даже главное магнитное поле Земли, являются сравнительно слабыми, и при их анализе практически пригодна модель «идеального ферромагнетика», не принимающая во внимание магнитное насыщение и магнитный гистерезис горных пород и руд;

2. все рассматриваемые поля, строго говоря, являются переменными, но относительно низкочастотными;

3. все рассматриваемые поля, строго говоря, неоднородны, но степень их неоднородности различна, и на практике предпочтение чаще оказывают источникам, создающим в объеме изучаемых объектов квазиоднородное поле.

Отмеченная общность и дает возможность создания общей теории интерпретации данных 
комплексной магниторазведки. В ее фундаменте лежит анализ аномального поля изотропного магнитного и проводящего шара, помещенного в однородное, гармонически меняющееся магнитное поле. Однако прежде чем приступить к решению данной задачи, рассмотрим более простую магнитостатическую задачу, другими словами, случай постоянного первичного магнитного поля. Данное вспомогательное решение поможет в дальнейшем анализировать основную задачу.

Как было отмечено, постоянное магнитное поле описывается двумя основными характеристиками: напряженностью $\overrightarrow{\mathrm{H}}$ и индукцией $\overrightarrow{\mathrm{B}}$, между которыми в системе СИ существует локальное соотношение (2.1). Подставив в (2.1) намагниченность, выраженную по формуле для «идеального ферромагнетика» (1.2), получим

$$
\overrightarrow{\mathrm{B}}=\mu_{0}\left[(1+\kappa) \overrightarrow{\mathrm{H}}+\overrightarrow{\mathrm{I}}_{\mathrm{n}}\right] \text {. }
$$

Величина в круглых скобках, очевидно, является относительной магнитной проницаемостью вещества $\mu$, и (3.1) можно переписать в следующем виде

$$
\overrightarrow{\mathrm{B}}=\mu_{0}\left(\mu \overrightarrow{\mathrm{H}}+\overrightarrow{\mathrm{I}}_{\mathrm{n}}\right) .
$$

Характеристики $\overrightarrow{\mathrm{H}}$ и $\overrightarrow{\mathrm{B}}$ удовлетворяют дифференциальным уравнениям (2.3). Как устанавливается в теории поля [5], из этих уравнений следует, что на границах тел непрерывны тангенциальная компонента напряженности и нормальная компонента индукции магнитного поля. Нормальная компонента напряженности на границе тела испытывает разрыв, равный нормальной компоненте намагниченности в данной точке. Эти соотношения являются исходными при решении прямых задач магниторазведки.

Для решения задачи о намагничении шара в однородном и постоянном магнитном поле

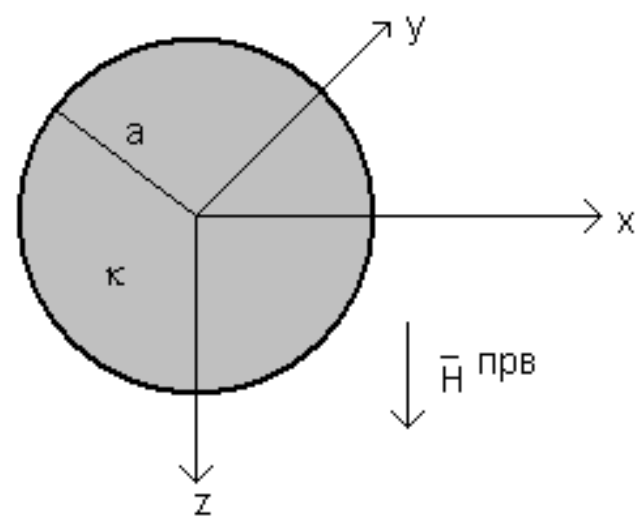

Рис. 7. К решению прямой задачи для однородного шара удобно ввести скалярный магнитный потенциал U. Возможность его введения вытекает из первого уравнения в (2.3). Определим его соотношением

$$
\overrightarrow{\mathrm{H}}(\mathrm{a})=-\operatorname{grad} \mathrm{U}(\mathrm{a}) \text {. }
$$

Знак минус перед градиентом связан с тем, что одноименные магнитные полюса (т.н. фиктивные магнитные массы) отталкиваются друг от друга. Подставим (3.3) во второе уравнение из (2.3) с учетом (3.2), в результате убедимся, что скалярный магнитный потенциал удовлетворяет следующему дифференциальному уравнению:

$$
\operatorname{div}(\mu \operatorname{grad} \mathrm{U})=\operatorname{div} \overrightarrow{\mathrm{I}}_{\mathrm{n}} .
$$

Для однородных объектов оно упрощается и переходит в уравнение Лапласа:

$$
\Delta \mathrm{U}=0 .
$$

На границах тел потенциал удовлетворяет двум граничным условиям. Из непрерывности тангенциальной компоненты напряженности следует, что потенциал на границе непрерывен. Если обозначить потенциал внутри тела как $U_{\mathrm{i}}$, а вне тела как $U_{\mathrm{e}}$, то на границе $U_{\mathrm{i}}=U_{\mathrm{e}}$. Данные потенциалы, очевидно, являются суммой потенциалов первичного и вторичного полей: $\mathrm{U}=\mathrm{U}^{\text {прв }}+\mathrm{U}^{\text {втр }}$, следовательно, можно считать, что на границе непрерывен вторичный потенциал, и первое граничное условие для рассматриваемой задачи принимает вид:

$$
\mathrm{U}_{\mathrm{i}}^{\text {прв }}=\mathrm{U}_{\mathrm{e}}^{\mathrm{BTp}} \text {. }
$$

Второе граничное условие вытекает из непрерывности нормальных компонент вектора индукции и, соответственно, имеет следующий вид:

$$
\frac{\partial \mathrm{U}^{\text {прв }}}{\partial \mathrm{n}}+\frac{\partial \mathrm{U}_{\mathrm{e}}^{\text {втр }}}{\partial \mathrm{n}}=\mu\left(\frac{\partial \mathrm{U}^{\text {прв }}}{\partial \mathrm{n}}+\frac{\partial \mathrm{U}_{\mathrm{i}}^{\mathrm{втр}}}{\partial \mathrm{n}}\right) .
$$


Ю.И. Блох

Решение поставленной задачи сводится к нахождению таких потенциалов внутри и вне шара, которые удовлетворяют дифференциальному уравнению и двум граничным условиям.

Пусть однородный и изотропный шар радиуса а с магнитной восприимчивостью $\kappa$ помещен в однородное первичное магнитное поле $\overrightarrow{\mathrm{H}}^{\text {прв}}$, направленное вдоль оси Oz. Расположим центр шара в начале декартовой системы координат (рис. 7) и определим потенциал первичного поля. С помощью (3.3) легко убедиться, что

$$
\mathrm{U}^{\text {Прв }}=-\mathrm{H}^{\text {Прв }} \mathrm{z} \text {. }
$$

Известно, что шар в однородном поле поляризуется однородно, а его внешнее поле эквивалентно полю диполя. Убедимся в этом, попытавшись удовлетворить условиям теоремы единственности теории поля. Для этого составим выражения для внутреннего и внешнего вторичных потенциалов с неопределенными коэффициентами и найдем их значения из граничных условий. Для удобства расчетов перейдем к сферическим координатам $\mathrm{R}, \theta, \varphi$, вводимым соотношениями:

$$
\mathrm{x}=\mathrm{R} \sin \theta \cos \varphi ; \mathrm{y}=\mathrm{R} \sin \theta \sin \varphi ; \mathrm{z}=\mathrm{R} \cos \theta .
$$

$\mathrm{B}$ сферической системе координат потенциал первичного поля принимает вид

$$
\mathrm{U}^{\text {прв }}=-\mathrm{H}^{\text {прв }} \mathrm{R} \cos \theta \text {. }
$$

Внутренний вторичный потенциал будем искать как потенциал однородного поля с неопределенным коэффициентом А:

$$
\mathrm{U}_{\mathrm{i}}^{\text {прв }}=\mathrm{A} \mathrm{H}^{\text {прв }} \mathrm{R} \cos \theta .
$$

Внешний вторичный потенциал будем искать в форме потенциала вертикального диполя, расположенного в центре шара, с неопределенным коэффициентом С:

$$
\mathrm{U}_{\mathrm{e}}^{\text {втр }}=\mathrm{C} \mathrm{H}^{\text {прв }} \frac{\cos \theta}{\mathrm{R}^{2}} \text {. }
$$

Удовлетворим сначала условие (3.6). При $\mathrm{R}=\mathrm{a}$ приравняем (3.11) и (3.12), в результате чего получим соотношение $\mathrm{C}=\mathrm{a}^{3} \mathrm{~A}$. В условии (3.7) вместо дифференцирования по нормали в сферической системе можно дифференцировать по координате $\mathrm{R}$, тогда получим следующее уравнение для определения A: $-1-2 A=\mu(-1+A)$ откуда $A=(\mu-1) /(\mu+2)$. Выражения для потенциалов, удовлетворяющие условиям теоремы единственности, примут вид:

$$
\begin{aligned}
& \mathrm{U}_{\mathrm{i}}^{\text {прв }}=\frac{\mu-1}{\mu+2} \mathrm{H}^{\text {прв }} \mathrm{R} \cos \theta, \\
& \mathrm{U}_{\mathrm{e}}^{\text {втр }}=\frac{\mu-1}{\mu+2} \mathrm{H}^{\text {прв }} \frac{\cos \theta}{\mathrm{R}^{2}} .
\end{aligned}
$$

Переходя обратно к декартовой системе координат, получим внутри шара

$$
\mathrm{U}_{\mathrm{i}}^{\text {втр }}=\frac{\mu-1}{\mu+2} \mathrm{H}^{\text {прв }} \mathrm{z} \text {, }
$$

а вне его

$$
\mathrm{U}_{\mathrm{e}}^{\text {втр }}=\frac{\mu-1}{\mu+2} \mathrm{a}^{3} \mathrm{H}^{\text {прв }} \frac{\mathrm{z}}{\left(\mathrm{x}^{2}+\mathrm{y}^{2}+\mathrm{z}^{2}\right)^{3 / 2}} .
$$

Проанализируем полученные выражения. Потенциал вне шара эквивалентен потенциалу диполя, помещенного в его центр, с дипольным моментом, ориентированным по направлению намагничивающего поля. Преобразуем (3.15), умножив и поделив на $4 \pi / 3$ и учитывая, что $\mu=1+\kappa$. В результате получим 
Ю.И. Блох

$$
\mathrm{U}_{\mathrm{e}}^{\text {втр }}=\frac{1}{4 \pi} \cdot \frac{\kappa \mathrm{H}^{\text {прв }}}{1+\frac{1}{3} \kappa} \cdot \frac{4 \pi \mathrm{a}^{3}}{3} \cdot \frac{\mathrm{z}}{\left(\mathrm{x}^{2}+\mathrm{y}^{2}+\mathrm{z}^{2}\right)^{3 / 2}} .
$$

Первый сомножитель в данной формуле возникает из-за применения системы СИ, второй сомножитель представляет собой намагниченность шара, а третий - его объем. Таким образом, потенциал внешнего аномального поля шара можно представить в виде

$$
\mathrm{U}_{\mathrm{e}}^{\text {втр }}=\frac{1}{4 \pi} \cdot \mathrm{IV} \cdot \frac{\mathrm{z}}{\left(\mathrm{x}^{2}+\mathrm{y}^{2}+\mathrm{z}^{2}\right)^{3 / 2}} .
$$

Дипольный момент шара, как видно, совпадает с его магнитным моментом M=IV, a намагниченность шара определяется формулой

$$
\mathrm{I}=\frac{\kappa \mathrm{H}^{\text {прв }}}{1+\frac{1}{3} \kappa} .
$$

Напомним, что задача решалась при $\mathrm{I}_{\mathrm{n}}=0$. Если учесть наличие остаточной намагниченности, направленной так же, как намагничивающее поле, то (3.18) преобразуется к виду

$$
\mathrm{I}=\frac{\kappa \mathrm{H}^{\text {прв }}+\mathrm{I}_{\mathrm{n}}}{1+\frac{1}{3} \kappa} \text {. }
$$

Вообще говоря, для любого трехосного эллипсоида, намагниченного вдоль одной из его осей, имеет место следующее соотношение:

$$
\mathrm{I}=\frac{\kappa \mathrm{H}^{\text {прв }}+\mathrm{I}_{\mathrm{n}}}{1+\kappa \mathrm{N}},
$$

где $\mathrm{N}$ - т.н. коэффициент размагничивания или размагничивающий фактор, следовательно, для шара $\mathrm{N}=1 / 3$.

Анализируя (3.14), легко установить, что потенциал внутреннего поля шара - это потенциал однородного поля с напряженностью

$$
\overrightarrow{\mathrm{H}}_{\mathrm{i}}^{\text {втр }}=-\mathrm{N} \overrightarrow{\mathrm{I}},
$$

направленного противоположно намагниченности, следовательно, в данном случае и первичному полю. Из вида полученной формулы становится ясно, почему внутреннее аномальное поле называют размагничивающим, а параметр $\mathrm{N}$ - коэффициентом размагничивания.

Для того чтобы получить выражения для компонент аномальной индукции шара, намагниченного в вертикальном первичном поле, применим к (3.15) соотношение (3.3) и умножим результат на $\mu_{0}$. В итоге получим:

$$
\begin{aligned}
& \mathrm{B}_{\mathrm{x}}=\mu_{0} \mathrm{a}^{3} \mathrm{H}^{\text {прв }} \frac{\mu-1}{\mu+2} \frac{3 \mathrm{xz}}{\left(\mathrm{x}^{2}+\mathrm{y}^{2}+\mathrm{z}^{2}\right)^{5 / 2}} \text {, } \\
& \mathrm{B}_{\mathrm{y}}=\mu_{0} \mathrm{a}^{3} \mathrm{H}^{\text {прв }} \frac{\mu-1}{\mu+2} \frac{3 y z}{\left(\mathrm{x}^{2}+\mathrm{y}^{2}+\mathrm{z}^{2}\right)^{5 / 2}} \text {, } \\
& \mathrm{B}_{\mathrm{z}}=\mu_{0} \mathrm{a}^{3} \mathrm{H}^{\text {прв }} \frac{\mu-1}{\mu+2} \frac{2 \mathrm{z}^{2}-\mathrm{x}^{2}-\mathrm{y}^{2}}{\left(\mathrm{x}^{2}+\mathrm{y}^{2}+\mathrm{z}^{2}\right)^{5 / 2}} .
\end{aligned}
$$

Теперь можно перейти к решению основной задачи о шаре, помещенном в однородное, гармонически меняющееся магнитное поле. Вообще говоря, задача о дифракции электромагнитной волны на шаре относится к фундаментальным в самых разнообразных 
областях, и ей, соответственно, посвящено огромное число работ. В достаточно общей постановке ее исследовали еще в начале XX века Густав Ми (Gustav Mie, 1869-1957) [249] и будущий нобелевский лауреат Петер Дебай (Peter Debye, 1884-1966), который, в 1908 г. защитил докторскую диссертацию по теме «Давление света на шары из произвольного материала» [238]. Применительно к геофизической проблематике, задача для шара в переменном магнитном поле впервые была рассмотрена в 1951 г. Джеймсом Уэйтом (J.R. Wait), который при этом отметил, что анализировал частный случай результатов П. Дебая для слабо проводящей вмещающей среды [259]. Именно эта модель и является базовой для комплексной магниторазведки - в дальнейшем она использовалась С. Уордом, Ю.В. Якубовским, А.Б. Великиным и Г.С. Франтовым, Б.С. Световым и др. При этом, как показала многолетняя практика, слабая проводимость и на самом деле оказывается присущей вмещающим средам в большинстве рудных районов.

Изложение решения данной задачи, тем не менее, мы будем вести не по статье Дж. Уэйта, а в соответствии с удобным и достаточно общим операторным подходом Б.С. Светова [153], восходящим к пионерской работе П. Дебая. Б.С. Светов справедливо отмечает, что применительно к рассматриваемой задаче решение уравнений Максвелла может быть сведено к решению двух отдельных скалярных задач для потенциалов Дебая электрического (u) и магнитного (v) типов [153, с. 409-410]. C помощью этих потенциалов суммарное поле разбивается на два отдельных поля. В поле магнитного типа отсутствует нормальная к поверхности раздела (здесь - радиальная) компонента электрического поля. В поле электрического типа, соответственно, отсутствует радиальная компонента магнитного поля.

Итак, пусть однородный и изотропный шар с магнитной проницаемостью $\mu$ (с магнитной восприимчивостью $\kappa)$, проводимостью $\gamma$ и диэлектрической постоянной $\varepsilon$ помещен в неограниченную с внешней стороны вмещающую среду с физическими свойствами $\mu_{\mathrm{e}}, \gamma_{\mathrm{e}}$ и $\varepsilon_{\mathrm{e}}$. Радиус шара, как и в магнитостатической задаче, обозначим через а.

В сферической системе координат потенциалы Дебая удовлетворяют уравнению Гельмгольца

$$
\frac{\partial^{2}(\mathrm{u}, \mathrm{v})}{\partial \mathrm{R}^{2}}+\frac{2}{\mathrm{R}} \frac{\partial(\mathrm{u}, \mathrm{v})}{\partial \mathrm{R}}+\frac{1}{\mathrm{R}^{2} \sin \theta} \frac{\partial}{\partial \theta}\left(\sin \theta \frac{\partial(\mathrm{u}, \mathrm{v})}{\partial \theta}\right)+\frac{1}{\mathrm{R}^{2} \sin ^{2} \theta} \frac{\partial^{2}(\mathrm{u}, \mathrm{v})}{\partial \varphi^{2}} \mathrm{k}^{2}(\mathrm{u}, \mathrm{v})=0,
$$

a на поверхности шара - граничным условиям вида:

$$
\begin{aligned}
\mu_{1} \mathrm{v}_{1} & =\mu_{2} \mathrm{v}_{2}, & \frac{\partial}{\partial \mathrm{R}}\left(\mathrm{Rv}_{1}\right) & =\frac{\partial}{\partial \mathrm{R}}\left(\mathrm{Rv}_{2}\right), \\
\gamma_{1} \mathrm{u}_{1} & =\gamma_{2} \mathrm{u}_{2}, & \frac{\partial}{\partial \mathrm{R}}\left(\mathrm{Ru}_{1}\right) & =\frac{\partial}{\partial \mathrm{R}}\left(\mathrm{Ru}_{2}\right) .
\end{aligned}
$$

Частными решениями уравнения (3.25), как показал П. Дебай, являются сферические волновые функции:

$$
\left.\begin{array}{l}
\cos m \varphi \\
\sin m \varphi
\end{array}\right\} P_{n}^{m}(\cos \theta)\left\{\begin{array}{l}
j_{n}(k R) \\
h_{n}(k R)
\end{array},\right.
$$

где $\mathrm{P}_{\mathrm{n}}^{\mathrm{m}}(\cos \theta)$ - присоединенные функции Лежандра, a

$$
\mathrm{j}_{\mathrm{n}}(\mathrm{kR})=\sqrt{\frac{\pi}{2 \mathrm{kR}}} \mathrm{J}_{\mathrm{n}+\frac{1}{2}}(\mathrm{kR}) \quad \text { и } \quad \mathrm{h}_{\mathrm{n}}(\mathrm{kR})=\sqrt{\frac{\pi}{2 \mathrm{kR}}} \mathrm{H}_{\mathrm{n}+\frac{1}{2}}^{(1)}(\mathrm{kR})
$$

- сферические функции Бесселя. Эти решения периодичны по $\varphi$ и конечны при $\theta=0$ и $\theta=\pi / 2$. Первое из них ограничено в начале координат, а второе удовлетворяет условию регулярности на бесконечности. Напряженности электрического и магнитного полей связаны с потенциалами Дебая следующими соотношениями: для потенциала u

$$
\mathrm{E}_{\mathrm{R}}=\frac{\partial^{2}(\mathrm{Ru})}{\partial \mathrm{R}^{2}}+\mathrm{k}^{2}(R \mathrm{u}), \quad \mathrm{E}_{\theta}=\frac{1}{\mathrm{R}} \frac{\partial^{2}(\mathrm{Ru})}{\partial \mathrm{R} \partial \theta}, \mathrm{E}_{\varphi}=\frac{1}{\mathrm{R} \sin \theta} \frac{\partial^{2}(\mathrm{Ru})}{\partial \mathrm{R} \partial \varphi}
$$




$$
\mathrm{H}_{\mathrm{R}}=0, \mathrm{H}_{\theta}=\gamma \frac{1}{\mathrm{R} \sin \theta} \frac{\partial(\mathrm{Ru})}{\partial \varphi}, \mathrm{H}_{\varphi}=-\gamma \frac{1}{\mathrm{R} \sin \theta} \frac{\partial(\mathrm{Ru})}{\partial \theta},
$$

а для потенциала $\mathrm{v}$

$$
\begin{gathered}
\mathrm{H}_{\mathrm{R}}=\frac{\partial^{2}(\mathrm{Rv})}{\partial \mathrm{R}^{2}}+\mathrm{k}^{2}(R \mathrm{v}), \quad \mathrm{H}_{\theta}=\frac{1}{\mathrm{R}} \frac{\partial^{2}(\mathrm{Rv})}{\partial \mathrm{R} \partial \theta}, \mathrm{H}_{\varphi}=\frac{1}{\mathrm{R} \sin \theta} \frac{\partial^{2}(\mathrm{Rv})}{\partial \mathrm{R} \partial \varphi}, \\
\mathrm{E}_{\mathrm{R}}=0, \quad \mathrm{E}_{\theta}=\mathrm{i} \omega \mu \frac{1}{\mathrm{R} \sin \theta} \frac{\partial(\mathrm{Rv})}{\partial \varphi}, \mathrm{E}_{\varphi}=-\mathrm{i} \omega \mu \frac{1}{\mathrm{R} \sin \theta} \frac{\partial(\mathrm{Rv})}{\partial \theta} .
\end{gathered}
$$

Как было отмечено, решение задачи будем вести в обобщенном операторном виде. Предположим, что первичное поле некоторого возбудителя представимо с помощью потенциалов Дебая в виде суммы сферических волновых функций:

$$
\begin{aligned}
& \mathrm{u}=\Gamma^{\jmath}\left\{\mathrm{j}_{\mathrm{n}}\left(\mathrm{k}_{\mathrm{e}} \mathrm{R}\right)\right\}=\sum_{\mathrm{n}=0}^{\infty} \sum_{\mathrm{m}=0}^{\infty} \mathrm{a}_{\mathrm{mn}}^{\ni}\left\{\begin{array}{l}
\cos \mathrm{m} \varphi \\
\sin \mathrm{m} \varphi
\end{array}\right\} \mathrm{P}_{\mathrm{n}}^{\mathrm{m}}(\cos \theta)\left\{\mathrm{j}_{\mathrm{n}}\left(\mathrm{k}_{\mathrm{e}} \mathrm{R}\right)\right\}, \\
& \mathrm{v}=\Gamma^{\mathrm{M}}\left\{\mathrm{j}_{\mathrm{n}}\left(\mathrm{k}_{\mathrm{e}} \mathrm{R}\right)\right\}=\sum_{\mathrm{n}=0}^{\infty} \sum_{\mathrm{m}=0}^{\infty} \mathrm{a}_{\mathrm{mn}}^{M}\left\{\begin{array}{c}
\cos \mathrm{m} \varphi \\
\sin \mathrm{m} \varphi
\end{array}\right\} \mathrm{P}_{\mathrm{n}}^{\mathrm{m}}(\cos \theta)\left\{\mathrm{j}_{\mathrm{n}}\left(\mathrm{k}_{\mathrm{e}} \mathrm{R}\right)\right\},
\end{aligned}
$$

где $\mathrm{a}^{3}$ и $\mathrm{a}^{\mathrm{M}}$ - постоянные, зависящие от вида возбудителя. Из (3.32) и (3.33) следует, что операторы $\Gamma^{3}$ и $\Gamma^{\mathrm{M}}$ не зависят от координаты $\mathrm{R}$ и не изменяются при переходе через границу раздела.

Будем искать потенциалы внутри шара в следующем виде:

$$
\mathrm{u}_{\mathrm{i}}=\Gamma^{\ni}\left\{\beta_{\mathrm{n}}(\gamma) \mathrm{j}_{\mathrm{n}}(\mathrm{kR})\right\}, \quad \mathrm{v}_{\mathrm{i}}=\Gamma^{\mu}\left\{\beta_{\mathrm{n}}(\mu) \mathrm{j}_{\mathrm{n}}(\mathrm{kR})\right\},
$$

a во внешней среде в виде:

$$
\mathrm{u}_{e}=\Gamma^{\ni}\left\{\mathrm{j}_{\mathrm{n}}\left(\mathrm{k}_{\mathrm{e}} \mathrm{R}\right)+\alpha_{\mathrm{n}}(\gamma) \mathrm{h}_{\mathrm{n}}\left(\mathrm{k}_{\mathrm{e}} \mathrm{R}\right)\right\}, \quad \mathrm{v}_{\mathrm{e}}=\Gamma^{M}\left\{\mathrm{j}_{\mathrm{n}}\left(\mathrm{k}_{\mathrm{e}} \mathrm{R}\right)+\alpha_{\mathrm{n}}(\mu) \mathrm{h}_{\mathrm{n}}\left(\mathrm{k}_{\mathrm{e}} \mathrm{R}\right)\right\} .
$$

Для определения функций отражения $\alpha_{n}(\gamma)$ и $\alpha_{n}(\mu)$ надо подставить (3.34) и (3.35) в граничные условия (3.26) и (3.27) и символически сократить операторы $\Gamma^{\ni}$ и $\Gamma^{\mathrm{M}}$. Решив уравнения, получим:

$$
\alpha_{n}(\gamma, \mu)=\frac{j_{n}\left(k_{e} a\right)}{h_{n}\left(k_{e} a\right)} D_{n}(\gamma, \mu),
$$

где

$$
\begin{aligned}
& \mathrm{D}_{\mathrm{n}}(\gamma)=\frac{1-\frac{\gamma_{e}}{\gamma}+\mathrm{k}_{\mathrm{e}} \mathrm{a} \bar{\jmath}_{\mathrm{n}}\left(\mathrm{k}_{\mathrm{e}} \mathrm{a}\right)-\frac{\gamma_{e}}{\gamma} \mathrm{ka} \bar{\jmath}_{\mathrm{n}}(\mathrm{ka})}{1-\frac{\gamma}{\gamma}+\mathrm{k}_{\mathrm{e}} \mathrm{a} \overline{\mathrm{h}}_{\mathrm{n}}\left(\mathrm{k}_{\mathrm{e}} \mathrm{a}\right)-\frac{\gamma_{e}}{\gamma} \mathrm{ka} \overline{\mathrm{\jmath}}_{\mathrm{n}}(\mathrm{ka})}, \\
& \mathrm{D}_{\mathrm{n}}(\mu)=\frac{1-\frac{\mu_{e}}{\mu}+\mathrm{k}_{\mathrm{e}} \mathrm{a} \bar{\jmath}_{\mathrm{n}}\left(\mathrm{k}_{\mathrm{e}} \mathrm{a}\right)-\frac{\mu_{e}}{\mu} \mathrm{ka} \bar{\jmath}_{\mathrm{n}}(\mathrm{ka})}{1-\frac{\mu_{e}}{\mu}+\mathrm{k}_{\mathrm{e}} \mathrm{a} \overline{\mathrm{h}}_{\mathrm{n}}\left(\mathrm{k}_{\mathrm{e}} \mathrm{a}\right)-\frac{\mu_{e}}{\mu} \mathrm{ka} \bar{\jmath}_{\mathrm{n}}(\mathrm{ka})} .
\end{aligned}
$$

Здесь $\overline{\mathrm{J}}_{\mathrm{n}}(\mathrm{z})=\mathrm{j}_{\mathrm{n}}^{\prime}(\mathrm{z}) / \mathrm{j}_{\mathrm{n}}(\mathrm{z})$ и $\overline{\mathrm{h}}_{\mathrm{n}}(\mathrm{z})=\mathrm{h}_{\mathrm{n}}^{\prime}(\mathrm{z}) / \mathrm{h}_{\mathrm{n}}(\mathrm{z})$.

Чтобы получить требуемые выражения для конкретных типов возбудителей, достаточно выразить их первичное поле с помощью потенциалов Дебая в форме (3.35) и определить тем самым вид операторов $\Gamma^{\ni}$ и $\Gamma^{\mathrm{M}}$. С полученными таким образом разнообразными выражениями можно познакомиться в монографии Б.С. Светова [153].

Нас же интересует простейший случай однородного поля. Будем считать внешнее первичное гармонически меняющееся магнитное поле направленным (как и в магнитостатической задаче) вдоль оси Oz, однородным и имеющим напряженность вида $\mathrm{H}^{\text {прв}} \cdot \mathrm{e}^{\mathrm{i} \omega \mathrm{t}}$. В данном случае допущение об однородности первичного поля фактически подразумевает, что длина волны этого поля во вмещающей среде значительно больше, нежели диаметр шара, другими словами, $\left|\mathrm{k}_{\mathrm{e}} \mathrm{a}\right| \rightarrow 0$. Для наиболее интересной в комплексной 
Ю.И. Блох

магниторазведке области малых параметров выражение (3.36) может быть, вообще говоря, записано в виде:

$$
\alpha_{n}(\gamma, \mu)=\frac{\left(k_{e} a\right)^{2 n+1}}{(2 n-1) ! !(2 n+1) ! !} D_{n}(\gamma, \mu)
$$

при этом функции $\mathrm{D}_{\mathrm{n}}(\gamma, \mu)$ остаются конечными. Из (3.39) следует быстрое убывание членов рядов, описывающих вторичные поля источников, с ростом их номера n, что, фактически, дает возможность ограничиваться только начальными членами рядов. Для однородного возбуждающего поля это в данном случае и вовсе дополнительно диктуется граничными условиями [58, 259].

\section{$\operatorname{Re} D(\mu), \operatorname{Im} D(\mu)$}

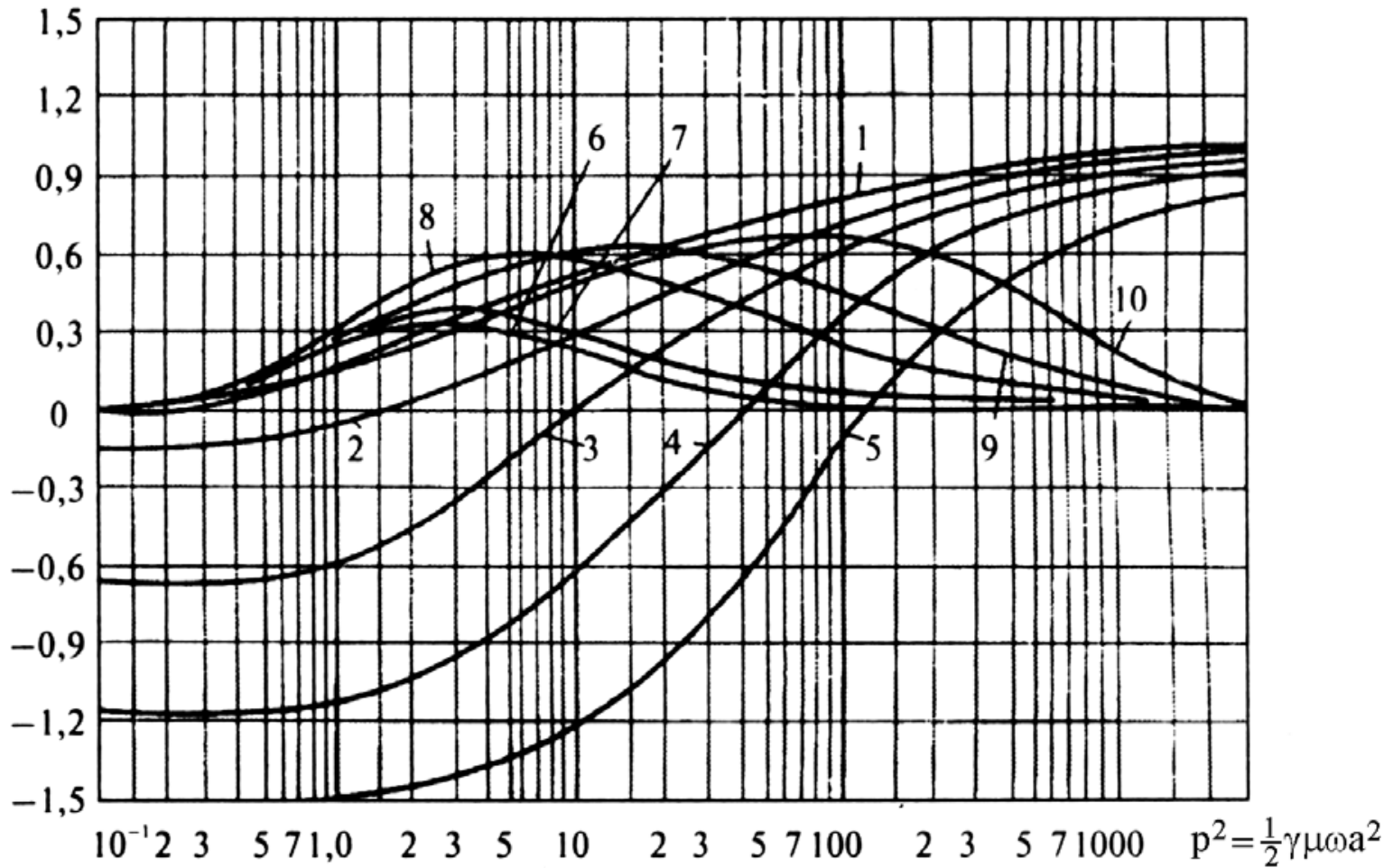

Рис. 8. Графики функции $\mathrm{D}(\mu)$ : на 1-5 - Re $\mathrm{D}(\mu)$, на 6-10 - $\operatorname{Im} \mathrm{D}(\mu)$. Кривым 1 и 6 соответствует $\mu / \mu_{\mathrm{e}}=1,0 ;$ кривым 2 и $7-\mu / \mu_{\mathrm{e}}=1,25 ;$ кривым 3 и $8-\mu / \mu_{\mathrm{e}}=2,5 ;$ кривым 4 и $9-\mu / \mu_{\mathrm{e}}=5,0 ;$ кривым 5 и $10-\mu / \mu_{\mathrm{e}}=10,0$ (по Б.С. Светову [152])

На рис. 8 показаны графики действительной и мнимой частей комплексной функции $\mathrm{D}(\mu)$, построенные в зависимости от параметра $\mathrm{p}^{2}=\gamma \mu \mathrm{aa}^{2} / 2$. Графики наглядно демонстрируют тот вытекающий из (3.38) факт, что в области низких частот (малых параметров $\mathrm{p}^{2}$ ) $\operatorname{Re} \mathrm{D}(\mu)$ выходит на отрицательную асимптоту [45]:

$$
\lim _{\mathrm{p} \rightarrow 0}[\operatorname{Re} \mathrm{D}(\mu)]=-2 \frac{\mu-\mu_{\mathrm{e}}}{\mu+2 \mu_{\mathrm{e}}},
$$

но при возрастании частоты Re $\mathrm{D}(\mu)$ переходит через нуль и на высоких частотах стремится к значению 1, никоим образом не зависящему от отношения магнитных проницаемостей и определяемому исключительно вихревыми токами, индуцированными в проводящем шаре. Мнимая часть - Im $\mathrm{D}(\mu)$ - слабо зависит от магнитной проницаемости шара, но ее экстремумы смещаются вдоль оси абсцисс пропорционально величине $\mu$, что, очевидно, является следствием того, что параметр $\mathrm{p}^{2}$ пропорционален $\mu$. 
При решении данной задачи не принималась во внимание граница раздела между Землей и воздухом, однако, надо учесть, что на практике во время съемки датчики полей практически всегда размещаются в воздухе, где $\mu_{\mathrm{e}}=1$. Практически наиболее важным для комплексной магниторазведки является именно тот случай, где вмещающая среда - немагнитна, и для него формула (3.40), описывающая низкочастотную асимптоту $\operatorname{Re} D(\mu)$, имеет очень простой вид:

$$
\lim _{\mathrm{p} \rightarrow 0}[\operatorname{Re} \mathrm{D}(\mu)]=-2 \frac{\mu-1}{\mu+2} .
$$

В результате оказывается, что компоненты амплитуды аномального поля шара, помещенного в однородное вертикальное магнитное поле, могут как для постоянного, так и для переменного, гармонически меняющегося первичного поля выражаться единообразно:

$$
\begin{aligned}
& B_{x}=-\mu_{0} \frac{a^{3} H^{\text {прв }}}{2} D(\mu) \frac{3 x z}{\left(x^{2}+y^{2}+z^{2}\right)^{5 / 2}}, \\
& B_{y}=-\mu_{0} \frac{a^{3} H^{\text {прв }}}{2} D(\mu) \frac{3 y z}{\left(x^{2}+y^{2}+z^{2}\right)^{5 / 2}}, \\
& B_{z}=-\mu_{0} \frac{a^{3} H^{\text {прв }}}{2} D(\mu) \frac{2 z^{2}-x^{2}-y^{2}}{\left(x^{2}+y^{2}+z^{2}\right)^{5 / 2}} .
\end{aligned}
$$

При $\omega \rightarrow 0$ эти выражения полностью совпадают с (3.22)-(3.24).

Таким образом, внешнее аномальное магнитное поле изотропного магнитного и проводящего шара, помещенного в однородное, гармонически меняющееся вертикальное магнитное поле, как и в магнитостатическом случае, эквивалентно полю магнитного диполя, помещенного в центр шара, момент которого коллинеарен приложенному полю. На низких частотах аномалия такого шара полностью совпадает с аномалией от шара, намагниченного в постоянном поле. При увеличении частоты возбуждающего поля аномалия над шаром сначала уменьшается по амплитуде, а затем меняет свой знак: на высоких частотах непосредственно над проводящим шаром наблюдается минимум аномального магнитного поля.

Выявленная закономерность и позволяет строить единую теорию интерпретации данных, получаемых в комплексной магниторазведке. При этом в качестве базового, как и в традиционной магниторазведке, оказывается необходимым рассматривать именно магнитостатическое приближение. Намагничивающие же поля, естественно, в общем случае следует рассматривать как неоднородные. 


\section{ГЛАВА 2. ПРЯМЫЕ ЗАДАЧИ КОМПЛЕКСНОЙ МАГНИТОРАЗВЕДКИ}

\section{§ 4. Основные подходы к решению прямых задач}

Решения прямых задач комплексной магниторазведки базируются на теории намагничения, истоки которой относятся к началу XIX в. и основоположниками классической версии которой стали Симеон Дени Пуассон (Siméon-Denis Poisson, 1781-1840) и Джордж Грин (George Green, 1793-1841). Пуассон обнародовал свой основной труд в этой области в 1824 г., а в 1828 г. была опубликована книга Грина. Ими независимо друг от друга было введено понятие идеального ферромагнетика, выведена формула, связывающая магнитный и гравитационный потенциалы однородно намагниченных тел, а также решены задачи о намагничивании шара и сферического слоя в однородном первичном поле. При этом Дж. Грин пошел еще дальше: ввел понятие «потенциальной функции», доказал знаменитые «формулы Грина» и на их основе пришел, говоря современным языком, к понятию потенциала простого слоя. В итоге ему удалось свести задачу о намагничении однородного тела фактически к решению интегрального уравнения для потенциала эквивалентного простого слоя, распределенного по его поверхности [242]. Этот метод до настоящего времени является одним из ведущих в изучении однородных тел, причем, за прошедшие без малого два века он многократно «переоткрывался» заново.

Исследования, последовавшие за пионерскими работами Пуассона и Грина, велись в двух направлениях: с одной стороны, решались частные задачи для простых тел, с другой стороны, велись поиски наиболее общих решений. Для первого направления наиболее значительны решения С. Пуассоном в 1837 г. и Ф. Нейманом в 1848 г. задачи о намагничении эллипсоида, а также решение, полученное Г. Кирхгофом в 1853 г., для бесконечного кругового цилиндра. В 1839 г. К. Гаусс предложил для построения эквивалентного простого слоя на поверхности тела использовать минимизацию функционала Дирихле, что по современным представлениям соответствует минимизации энергии магнитного поля.

Первой попыткой достаточно общего решения задачи, поставленной Пуассоном и Грином, была работа А. Бэра 1865 г., в которой для решения интегрального уравнения Пуассона-Грина предлагался метод последовательных приближений. В 1877 г. К. Нейман применил этот метод для решения задач о намагничении тел, причем, ему пришлось наложить ограничения на сходимость в зависимости от магнитной восприимчивости тела и его формы. Гораздо позже, в 1934 г. А.С. Случановский построил алгоритм последовательных приближений для произвольной поверхности Ляпунова и доказал его сходимость без столь сильных ограничений на восприимчивость [155]. Численная реализация предложенных методов была для тех времен крайне затруднительной, а строгие решения тогда удалось получить лишь для уединенных эллипсоидов, которые намагничиваются в однородном поле однородно.

Развитие магнитного метода изучения геологических объектов требовало решения прямой задачи, хотя бы приближенного, и для неэллипсоидальных тел. К началу XX в. интерпретация магнитных аномалий базировалась преимущественно на моделях идеальных стержнеобразных магнитов, как это представляли шведы Р. Тален и Т. Дальблом, а также на линейных однополюсных аномалиях, связанных с полубесконечными пластами, что предложил применять американец Г.Л. Смит [6]. Решающие шаги в создании традиционной концепции намагничения геологических объектов были сделаны венгром Лораном Этвёшем (Loránd Eötvös, 1848-1919) и шведом Вильгельмом Карльгейм-Гилленшельдом (Vilhelm CarlheimGyllensköld, 1859-1934). В 1906 г. Л. Этвёш предложил при вычислении магнитных аномалий геологических объектов пренебрегать зависимостью намагниченности от формы тел и считать тела любых форм однородно намагниченными. Это упрощение дало возможность Л. Этвёшу применять формулу Пуассона о связи гравитационного и магнитного потенциалов для объектов сложных форм, хотя строго справедливой она является лишь для уединенных эллипсоидов. С той поры и вплоть до настоящего времени упрощенная концепция намагничения является базой, на которой строится теория интерпретации. В связи с тем, что среди изучаемых тогда 
магнитных объектов преобладали магнетитовые руды, для которых полное пренебрежение зависимостью намагниченности от формы тел уже в начале прошлого века не представлялось возможным, В. Карльгейм-Гилленшельд дополнил концепцию Л. Этвёша. В 1910 г. он предложил для сильномагнитных объектов считать допустимой модель однородного намагничения, но учитывать размагничивание под действием внутреннего аномального поля, хотя бы приближенно. Для этого он аппроксимировал изучаемый объект эллипсоидом и вычислял его среднюю намагниченность, применяя коэффициенты размагничивания эквивалентного эллипсоида.

Таким образом, к 1910 году оформилась традиционная для магниторазведки упрощенная до крайности концепция намагничивания геологических объектов, основные черты которой сводятся к следующему.

1. Все геологические объекты независимо от их формы и величины магнитной восприимчивости считаются намагничивающимися в однородном поле однородно, вследствие чего для вычисления аномальных полей формально привлекается соотношение Пуассона о связи гравитационного и магнитного потенциалов, справедливое, строго говоря, лишь для уединенных эллипсоидов.

2. Если магнитная восприимчивость геологических объектов не превышает 0,125 ед. СИ (0,01 СГС), то при вычислении намагниченности эффект размагничивания под действием внутреннего аномального поля не учитывается вообще, поскольку его влияние, не превышающее в указанном диапазоне 12,5 \%, считается пренебрежимо малым.

3. Если магнитная восприимчивость геологических объектов больше 0,125 ед. СИ, то при вычислении их эффективной однородной намагниченности размагничивание учитывается приближенно на эквивалентном эллипсоиде.

Традиционная концепция, несмотря на ее сверхупрощенность и противоречия с теоретическими и экспериментальными результатами, и в настоящее время безоговорочно принимается многими исследователями, а на ее основе до сих пор решается большинство задач магниторазведки. Тем не менее, для комплексной магниторазведки, ориентированной на изучение среднемагнитных и сильномагнитных геологических объектов, подобное упрощение необоснованно. Что касается интерпретации результатов современных прецизионных измерений, концепцию вообще нельзя считать адекватной.

В 50-х годах прошлого века А.Г. Калашниковым были проведены тщательные экспериментальные исследования намагничивания призматических моделей [99-101]. Ему удалось показать, что призмы в однородном поле намагничиваются существенно неоднородно, и это приводит к качественному изменению форм графиков элементов аномального магнитного поля над ними. Оценив влияние такого изменения на результаты применения метода касательных, он пришел к выводу, что «приемы расчета глубины залегания и формы возмущающих тел на основе магниторазведочных наблюдений требуют дальнейших уточнений и разработки в отношении неоднородно намагниченных тел, имеющих как остаточное, так и индуктивное намагничение» [101, с. 1341].

Используя данные этих экспериментов, Е.Н. Мохова предприняла попытку теоретически проанализировать намагничение призмы с постоянной магнитной восприимчивостью [133, 134]. Несмотря на предельную упрощенность анализа, ей удалось получить определенные результаты, которыми воспользовались В.М. Девицын, М.И. Лапина и Б.Л. Шнеерсон [76]. Они оценили влияние неоднородности намагничения пласта железистых кварцитов на результаты методов характерных точек и касательных и пришли к выводу, что ее недоучет может приводить к погрешностям в определении глубин источников в 10-20\%. При этом было отмечено, что даже с учетом неоднородности намагничения объяснить наблюденную аномалию на месторождении Щигры в районе КМА не удается, и значительный вклад в нее наверняка вносит остаточная намагниченность.

Современный этап развития теории намагничения наступил с появлением электронновычислительных машин. Компьютеры дали возможность быстрой и эффективной реализации алгоритмов, что привело к значительному прогрессу в разработке методов вычисления 
магнитных полей. Основные успехи при этом были достигнуты с помощью методов интегральных уравнений, иначе говоря, на базе физической концепции, названной О.В. Тозони «концепцией вторичных источников» [190]. Согласно ей, расчет поля в пространстве, заполненном неоднородным, анизотропным и даже нелинейным магнетиком, может быть сведен к расчету поля в однородном изотропном пространстве от заданных первичных, а также и связанных вторичных - объемных и поверхностных источников. В зависимости от типов определяемых вторичных источников задача сводится к решению различных интегральных и интегро-дифференциальных уравнений. В рамки данной концепции органично вписывается и восходящий к Дж. Грину классический подход, сводящийся к построению на границах раздела сред эквивалентных простых слоев, который затем был весьма глубоко проработан Г.А. Гринбергом [73]. Однако, как оказалось, можно успешно применять также построение эквивалентных двойных слоев на границах и, главное, строить объемные распределения намагниченности.

Первые успехи в этом направлении связаны с работами, опубликованными в 1962-1963 гг. шведским геофизиком Андреашем Фогелем [256-258]. Для решения прямой задачи он использовал интегро-дифференциальное уравнение для намагниченности, распределенной по объему изучаемого тела, которое предлагал решать методом последовательных приближений. Метод Фогеля, по его собственным оценкам, обладал медленной сходимостью, поэтому не был широко внедрен в практику расчетов. В 1971 г. болгарские геофизики Д.П. Зидаров и П.К. Иванова модифицировали метод А. Фогеля на случай нелинейного магнетика, обладающего гистерезисом, но эта модификация лишь ухудшила сходимость.

Следующий шаг в развитии методов расчетов магнитных полей на компьютерах был сделан независимо несколькими исследователями, работающими как в геофизике, так и в электротехнике: в 1964 г. И.И. Пеккером [145], в 1966 г. П. Шармой [251] и в 1968 г. В.В. Соболевым и В.Т. Белоголовым [156]. В отличие от А. Фогеля, они предложили решать объемное интегро-дифференциальное уравнение для намагниченности путем сведения его к системе линейных алгебраических уравнений. Таким образом, записав в явном виде систему $3 n$ линейных уравнений, где $\mathrm{n}$ - число элементарных тел, аппроксимирующих изучаемый объект, указанные авторы решали ее методами Гаусса либо окаймления. Работавший в области электротехники, И.И. Пеккер дополнительно вводил нелинейную характеристику намагничения материала магнитопроводов, что привело его к необходимости организации внешнего итерационного цикла учета нелинейности. Данные алгоритмы получили широкое распространение.

В 1969 г. В.А. Филатов предложил алгоритм решения двумерной прямой задачи магниторазведки и метода искусственного подмагничивания, базирующийся на решении интегрального уравнения для плотности эквивалентного простого слоя на поверхности однородного тела [200]. Решение осуществлялось р-шаговым методом скорейшего спуска. Поскольку рассматривалась двумерная задача, в качестве источника первичного поля в методе искусственного подмагничивания анализировался бесконечно длинный кабель. Результаты, полученные В.А. Филатовым, сыграли важную рель в становлении метода искусственного подмагничивания.

Классическое направление теории намагничения получило новый импульс к развитию в начале 70-х годов. В 1971 г. киевские электротехники И.Д. Маергойз и О.В. Тозони предложили использовать для расчета магнитных полей эквивалентные двойные слои на поверхности однородных тел и вывели интегральное уравнение, обладавшее по сравнению с уравнением Пуассона-Грина рядом преимуществ, в том числе более высокой скоростью сходимости итерационных методов его решения [126]. В 1973 г. аналогичную идею применительно к задачам геофизики независимо предложил Г.М. Воскобойников [66]. В развитии подхода Г.М. Воскобойникова в дальнейшем участвовали Ю.М. Гуревич, А.В. Цирульский, П.С. Мартышко и ряд других исследователей [67]. За рубежом к исследованию возможностей применения двойных слоев при расчете магнитных аномалий обращался, в частности, М. Гвождара [244]. И.Д. Маергойз, стремясь к универсализации алгоритмов, рассчитанных на 
изучение неоднородных, анизотропных и нелинейных магнетиков, существенно продвинулся в их анализе путем одновременного построения вторичных источников и на границе объектов, и в их объеме. Результаты этих исследований были подытожены в его монографии 1979 г. [125].

Важные теоретические результаты, касающиеся намагничения геологических объектов, были получены путем сведения магнитостатической задачи к математической задаче линейного сопряжения аналитических функций. Впервые эквивалентность этих задач в 1974 г. показал А.В. Цирульский [215], получив интересные результаты по вопросам теории метода искусственного подмагничивания. В дальнейшем аналогичным путем шел в своих исследованиях П.С. Мартышко [129]. Необходимо отметить, что аналитические решения этим способом, к сожалению, можно получить лишь в ряде простых случаев, общих же алгоритмов решения прямой задачи на базе методов линейного сопряжения построено не было.

В 1974 г. автор предложил новый подход к численному решению задач магнитостатики, преобразовав интегро-дифференциальное уравнение для намагниченности, распределенной по объему объекта, в интегральное путем стирания слабой особенности и совместив решение проблемы аппроксимации объекта с вычислением намагниченности. Разработанный итерационный подход дал возможность более чем на порядок сократить время вычислений по сравнению с подходами Пеккера, Шармы, Соболева и Белоголова, причем, для его реализации, не требовался большой объем оперативной памяти компьютера.

К середине 70-х годов выяснилась необходимость разработки специальной методики решения прямой задачи магниторазведки для анизотропных геологических объектов. Массовые определения магнитной восприимчивости пород и руд показали ее существенно анизотропный характер. Особенно важной эта проблема является для месторождений метаморфогенной железорудной формации, представленной обычно магнетитовыми и гематито-магнетитовыми кварцитами - сырья, с которым связана большая часть мировых запасов железа. Огромную работу по изучению магнитных свойств железистых кварцитов кривого Рога и КМА провели 3.А. Крутиховская, В.Н. Завойский и другие [117-118]. В результате В.Н. Завойский создал методику вычисления магнитных аномалий от анизотропных объектов без учета размагничивания [84]. Показанное им, существенное влияние анизотропии на магнитные поля побудило автора заняться разработкой методики учета размагничивания при решении подобных задач. Для двумерных объектов алгоритм был создан в 1978 г., для трехмерных - в 1984 г.

Практически одновременно с публикацией автора, посвященной алгоритму для двумерной задачи [24], в 1980 г. появилась статья финских геофизиков Л. Эскола и Т. Терво [240] по модификации уравнения Пуассона-Грина для анизотропных тел. В дальнейшем это направление было продвинуто ими дальше в совместных работах с петербургскими геофизиками. Однако поскольку в этом подходе применяется эквивалентный простой слой на поверхности тела, алгоритм Эскола и Терво может иметь весьма ограниченное применение. Его можно использовать лишь тогда, когда магнитная восприимчивость однородна. Применительно к анизотропным телам это означает, что величина главных компонент тензора магнитной восприимчивости и ориентировка эллипсоида анизотропии в пространстве должны быть неизменными при решении задачи с помощью эквивалентного простого слоя. Фактически это соблюдается лишь для совокупности прямых пластов. Даже для уединенного пласта, образующего складку, такой подход не применим.

В 1998-99 гг. Н.Н. Винничук, Н.П. Костров и А.Н. Ратушняк вновь обратились к идее решения объемного интегро-дифференциального уравнения, которое ранее использовали Пеккер, Шарма, Соболев и Белоголов. Основными отличиями в их подходе стали использование при аппроксимации автоматизированной триангуляции и оптимизация вычисления тензора Грина для элементарных объемов различающихся форм [63]. С помощью своих программ указанные авторы изучили несколько любопытных теоретических моделей, в частности, проанализировали влияние эффекта размагничивания на поле сильномагнитного рельефа. Потом, однако, исследования фактически были свернуты, хотя отдельные результаты, 
в том числе, по испытанию сингулярного разложения при численном решении интегродифференциального уравнения продолжают публиковаться Н.П. Костровым [246].

В последние годы начал возрастать интерес зарубежных геофизиков к изучению сильномагнитных объектов, что проявилось в публикации ряда статей, но принципиально новых подходов к решению прямых задач в них фактически нет.

\section{§ 5. Объемное интегральное уравнение для намагниченности}

Вначале рассмотрим объемное интегро-дифференциальное уравнение для намагниченности, которое, как отмечалось в предыдущем параграфе, широко применяется для решения прямых задач, в том числе, в магнитостатике. При этом будем полагать, что изучаемый объект является неоднородным и анизотропным.

Пусть в неоднородное первичное поле с напряженностью $\overline{\mathrm{H}}^{\text {прв }}(\mathrm{a})$, заданное в немагнитной однородной среде, внесено неоднородное тело $\mathrm{V}$ с магнитной восприимчивостью $\kappa(\mathrm{a})$ и естественной остаточной намагниченностью $\overline{\mathrm{I}}_{\mathrm{n}}(\mathrm{a})$, ограниченное поверхностью S. Тогда в объеме тела к полю $\overline{\mathrm{H}}^{\text {прв }}\left(\right.$ а)прибавится поле $\overline{\mathrm{H}}^{\text {втр }}(\mathrm{a})$, возникающее под действием поляризации, и намагничивание тела будет происходить под действием суммарного поля

$$
\overline{\mathrm{H}}(\mathrm{a})=\overline{\mathrm{H}}^{\text {прв }}(\mathrm{a})+\overline{\mathrm{H}}^{\text {втр }}(\mathrm{a}) \text {. }
$$

Согласно принятой модели намагничения (1.2), для анизотропного идеализированного ферромагнетика в некоторой точке внутри тела намагниченность $\overline{\mathrm{I}}(\mathrm{a})$ будет описываться следующим образом:

$$
\overline{\mathrm{I}}(\mathrm{a})=\kappa(\mathrm{a})\left[\overline{\mathrm{H}}^{\mathrm{пp}}(\mathrm{a})+\overline{\mathrm{H}}^{\mathrm{BTp}}(\mathrm{a})\right]+\overline{\mathrm{I}}_{\mathrm{n}}(\mathrm{a}) .
$$

Поле $\overline{\mathrm{H}}^{\text {втр }}$ является функцией намагниченности, следовательно, выражая его через намагниченность в виде интеграла по объему тела [39], то есть

$$
\overline{\mathrm{H}}^{\text {втр }}(\mathrm{a})=\frac{1}{4 \pi} \operatorname{grad}_{\mathrm{a}} \int_{\mathrm{V}}\left(\overline{\mathrm{I}}(\mathrm{q}) \operatorname{grad}_{\mathrm{a}} \frac{1}{\mathrm{~L}_{\mathrm{qa}}}\right) \mathrm{dV},
$$

приходим к следующему интегро-дифференциальному уравнению:

$$
\overline{\mathrm{I}}(\mathrm{a})=\kappa(\mathrm{a})\left[\overline{\mathrm{H}}^{\text {прв }}(\mathrm{a})+\frac{1}{4 \pi} \operatorname{grad}_{\mathrm{a}} \int_{\mathrm{V}}\left(\overline{\mathrm{I}}(\mathrm{q}) \operatorname{grad}_{\mathrm{a}} \frac{1}{\mathrm{~L}_{\mathrm{qa}}}\right) \mathrm{dV}\right]+\overline{\mathrm{I}}_{\mathrm{n}}(\mathrm{a}),
$$

где $\mathrm{L}_{\mathrm{qa}}$ - расстояние от текущей точки q тела $\mathrm{V}$ до точки $\mathrm{a}$; оператор $\operatorname{grad}_{\mathrm{a}}$ означает, что дифференцирование проводится по координатам точки а. При $\mathrm{q} \rightarrow \mathrm{a}$, очевидно, $\mathrm{L}_{\text {qа }} \rightarrow 0$ и это означает, что уравнение (5.4) имеет под интегралом особенность (сингулярность), соответственно, является сингулярным интегральным уравнением.

Введем обозначение первичной намагниченности тела

$$
\overline{\mathrm{I}}_{0}(\mathrm{a})=\kappa(\mathrm{a}) \overline{\mathrm{H}}^{\text {прв }}(\mathrm{a})+\overline{\mathrm{I}}_{\mathrm{n}}(\mathrm{a}),
$$

пригодное и для изотропных, и для анизотропных объектов, тогда (5.4) можно переписать в виде:

$$
\overline{\mathrm{I}}(\mathrm{a})=\overline{\mathrm{I}}_{0}(\mathrm{a})+\frac{1}{4 \pi} \kappa(\mathrm{a}) \operatorname{grad}_{\mathrm{a}} \int_{\mathrm{V}}\left(\overline{\mathrm{I}}(\mathrm{q}) \operatorname{grad}_{\mathrm{a}} \frac{1}{\mathrm{~L}_{\mathrm{qa}}}\right) \mathrm{dV} .
$$

В традиционной концепции намагничения при вычислении намагниченности пренебрегают вторым слагаемым в данном уравнении и считают $\overline{\mathrm{I}}(\mathrm{a})=\overline{\mathrm{I}}_{0}(\mathrm{a})$, что для сильномагнитных объектов может приводить к ошибкам при интерпретации. Именно в полном виде уравнения (5.4) и (5.6) удовлетворяют как системе исходных дифференциальных уравнений поля, так и граничным условиям, что показано Д.П. Зидаровым [264] и И.И. Пеккером [145]. 
Ю.И. Блох

Рассмотрим в качестве примера решение уравнения (5.6) для однородного анизотропного шара в однородном поле. Полагая $\mathrm{I}=\mathrm{CI}_{0}$, где $\mathrm{C}$ - неопределенная постоянная, получим из (5.6):

$$
\mathrm{C} \overline{\mathrm{I}}_{0}=\overline{\mathrm{I}}_{0}+\frac{\mathrm{C}}{4 \pi} \kappa \operatorname{grad}_{\mathrm{a}} \int_{\mathrm{V}}\left(\overline{\mathrm{I}}_{0} \operatorname{grad}_{\mathrm{a}} \frac{1}{\mathrm{~L}_{\text {qa }}}\right) \mathrm{dV} .
$$

Как известно, для потенциала внутри однородного шара [39]:

$$
\int_{\mathrm{V}} \frac{\mathrm{dV}}{\mathrm{L}_{\mathrm{qa}}}=\frac{3 \mathrm{~V}}{2 \mathrm{R}_{\mathrm{m}}}\left(1-\frac{\mathrm{R}^{2}}{3 \mathrm{R}_{\mathrm{II}}^{2}}\right),
$$

где $\mathrm{V}=\frac{4}{3} \pi \mathrm{R}_{\text {ш }}^{3}$ - объем шара, $\mathrm{R}_{\text {ш }}$ - его радиус, a $\mathrm{R}$ - расстояние от центра шара до точки, в которой вычисляется потенциал. С учетом данной формулы (5.6) можно переписать в виде

$$
\mathrm{C} \overline{\mathrm{I}}_{0}=\overline{\mathrm{I}}_{0}-\frac{\mathrm{C}}{3} \kappa \overline{\mathrm{I}}_{0}
$$

или

$$
\mathrm{C}\left(3 \overline{\mathrm{I}}_{0}+\kappa \overline{\mathrm{I}}_{0}\right)=3 \overline{\mathrm{I}}_{0}
$$

Отсюда следует

$$
\mathrm{C}[3 \mathrm{E}+\kappa] \overline{\mathrm{I}}_{0}=3 \overline{\mathrm{I}}_{0}
$$

И

$$
\overline{\mathrm{I}}=\mathrm{C} \overline{\mathrm{I}}_{0}=3[3 \mathrm{E}+\kappa]^{-1} \overline{\mathrm{I}}_{0},
$$

где $[3 \mathrm{E}+\kappa]^{-1}-$ матрица, обратная к $[3 \mathrm{E}+\kappa]$. Таким образом, намагниченность однородного анизотропного шара можно найти по формуле

$$
\overline{\mathrm{I}}=3[3 \mathrm{E}+\kappa]^{-1}\left(\kappa \overline{\mathrm{H}}^{\text {прв }}+\overline{\mathrm{I}}_{\mathrm{n}}\right) .
$$

Для изотропного шара, когда $\kappa$ - скаляр, формула упрощается и принимает вид, соответствующий (3.20):

$$
\overline{\mathrm{I}}=\frac{\kappa \overline{\mathrm{H}}^{\text {прв }}+\overline{\mathrm{I}}_{\mathrm{n}}}{1+\mathrm{N} \kappa},
$$

где $\mathrm{N}=1 / 3$ - коэффициент размагничивания шара в системе СИ. Две последние формулы понадобятся нам в дальнейшем.

Как уже отмечалось, при численном решении интегро-дифференциального уравнения для намагниченности применялись два подхода. И.И. Пеккер, П. Шарма, В.В. Соболев и В.Т. Белоголов разбивали изучаемый объем на $\mathrm{n}$ элементарных тел (параллелепипедов или многогранников) и, полагая намагниченность каждого из элементов однородной, приходили к системе 3n линейных алгебраических уравнений для компонент эффективной намагниченности элементов. Эту систему решали на компьютерах прямыми способами, такими как метод Гаусса или метод окаймления. Основной недостаток данного подхода состоит в том, что для его реализации требуются огромные объемы оперативной памяти компьютера.

Другой подход к решению интегро-дифференциального уравнения для намагниченности состоит в применении метода последовательных приближений. При этом матрица системы линейных алгебраических уравнений в памяти компьютера не хранится, но требуется перевычисление входящих в нее коэффициентов на каждой из итераций. Такой подход в магниторазведке применяли А. Фогель, Д.П. Зидаров и П.К. Иванова. Преимущества итерационного подхода, тем не менее, могут быть сведены на нет в силу ряда причин, главная из которых состоит в интегро-дифференциальном характере уравнения (5.6) с сингулярностью при q $\rightarrow$ a. Из-за этого сходимость итерационного процесса не высока, что требует большого числа итераций и не всегда приводит, как показал А. Фогель [258], к нужному результату. Большое число итераций означает большое машинное время, затрачиваемое на вычисления, ведь при аппроксимации объекта параллелепипедами для определения каждого из коэффициентов надо 8 раз рассчитать арктангенсы или логарифмы рациональных дробей. В 
Ю.И. Блох

связи с этим итерационные методы решения интегро-дифференциального уравнения не нашли широкого применения.

Таким образом, на пути создания эффективного алгоритма решения прямой задачи необходимо преодолеть затруднения, связанные с сингулярностью в уравнении (5.6) и с трудоемкостью вычисления коэффициентов, характеризующих поля параллелепипедов или многогранников. Эти затруднения можно преодолеть путем перехода от интегродифференциального к интегральному уравнению для намагниченности, вычисления полей изометричных элементов по формулам для эквивалентных шаров или цилиндров, а также за счет разработки процедуры учета попарного взаимовлияния элементов.

Чтобы перейти к интегральному уравнению, в исходном интегро-дифференциальном уравнении надо стереть слабую особенность, для чего можно выделить малый шар $\mathrm{V}_{\mathrm{a}} \mathrm{c}$ центром в точке а, тогда уравнение (5.6) примет следующий вид:

$$
\begin{aligned}
\overline{\mathrm{I}}(\mathrm{a})= & \overline{\mathrm{I}}_{0}(\mathrm{a})+\frac{1}{4 \pi} \kappa(\mathrm{a}) \operatorname{grad}_{\mathrm{a}} \int_{\mathrm{V}_{\mathrm{a}}}\left(\overline{\mathrm{I}}(\mathrm{q}) \operatorname{grad}_{\mathrm{a}} \frac{1}{\mathrm{~L}_{\mathrm{qa}}}\right) \mathrm{dV}+ \\
& +\frac{1}{4 \pi} \kappa(\mathrm{a}) \operatorname{grad}_{\mathrm{a}} \int_{\mathrm{V}-\mathrm{V}_{\mathrm{a}}}\left(\overline{\mathrm{I}}(\mathrm{q}) \operatorname{grad}_{\mathrm{a}} \frac{1}{\mathrm{~L}_{\mathrm{qa}}}\right) \mathrm{dV} .
\end{aligned}
$$

Так как шар $\mathrm{V}_{\text {a }}$ мал, его можно считать намагниченным однородно, при этом, учтя, что в системе СИ коэффициент размагничивания шара $\mathrm{N}=1 / 3$, получим

$$
\frac{1}{4 \pi} \operatorname{grad}_{\mathrm{a}} \int_{\mathrm{V}_{\mathrm{a}}}\left(\overline{\mathrm{I}}(\mathrm{q}) \operatorname{grad}_{\mathrm{a}} \frac{1}{\mathrm{~L}_{\mathrm{qa}}}\right) \mathrm{dV}=-\frac{1}{3} \overline{\mathrm{I}}(\mathrm{a}),
$$

тогда (5.15) преобразуется следующим образом:

$$
\overline{\mathrm{I}}(\mathrm{a})=\overline{\mathrm{I}}_{0}(\mathrm{a})-\frac{1}{3} \overline{\mathrm{I}}(\mathrm{a})+\frac{1}{4 \pi} \kappa(\mathrm{a}) \operatorname{grad}_{\mathrm{a}} \int_{\mathrm{V}-\mathrm{V}_{\mathrm{a}}}\left(\overline{\mathrm{I}}(\mathrm{q}) \operatorname{grad}_{\mathrm{a}} \frac{1}{\mathrm{~L}_{\mathrm{qa}}}\right) \mathrm{dV} .
$$

Собирая члены, содержащие İ(a) в левую часть, придем к следующему уравнению:

$$
\left[\mathrm{E}+\frac{1}{3} \kappa(\mathrm{a})\right] \overline{\mathrm{I}}(\mathrm{a})=\overline{\mathrm{I}}_{0}(\mathrm{a})+\frac{1}{4 \pi} \kappa(\mathrm{a}) \operatorname{grad}_{\mathrm{a}} \int_{\mathrm{V}-\mathrm{V}_{\mathrm{a}}}\left(\overline{\mathrm{I}}(\mathrm{q}) \operatorname{grad}_{\mathrm{a}} \frac{1}{\mathrm{~L}_{\mathrm{qa}}}\right) \mathrm{dV} .
$$

Поскольку сингулярность под интегралом ликвидирована, операции дифференцирования и интегрирования можно поменять местами:

$$
\left[\mathrm{E}+\frac{1}{3} \kappa(\mathrm{a})\right] \overline{\mathrm{I}}(\mathrm{a})=\overline{\mathrm{I}}_{0}(\mathrm{a})+\frac{1}{4 \pi} \kappa(\mathrm{a}) \int_{\mathrm{V}-\mathrm{V}_{\mathrm{a}}} \operatorname{grad}_{\mathrm{a}}\left(\overline{\mathrm{I}}(\mathrm{q}) \operatorname{grad}_{\mathrm{a}} \frac{1}{\mathrm{~L}_{\mathrm{qa}}}\right) \mathrm{dV} .
$$

Преобразуем подынтегральное выражение, для чего воспользуемся известной формулой векторного анализа, характеризующей градиент скалярного произведения двух векторов:

$$
\operatorname{grad}(\overline{\mathrm{P}} \cdot \overline{\mathrm{Q}})=\frac{\mathrm{d} \overline{\mathrm{P}}}{\mathrm{d} \overline{\mathrm{r}}} \overline{\mathrm{Q}}+\frac{\mathrm{d} \overline{\mathrm{Q}}}{\mathrm{d} \overline{\mathrm{r}}} \overline{\mathrm{P}}+[\overline{\mathrm{P}} \times \operatorname{rot} \overline{\mathrm{Q}}]+[\overline{\mathrm{Q}} \times \operatorname{rot} \overline{\mathrm{P}}],
$$

где производные $\mathrm{d} \overline{\mathrm{P}} / \mathrm{d} \bar{r}$ и $\mathrm{d} \overline{\mathrm{Q}} / \mathrm{d} \bar{r}$ представляют собой линейные операторы (аффиноры), которые можно выразить в матричной форме. Если, например, $\overline{\mathrm{P}}=(\mathrm{X}, \mathrm{Y}, \mathrm{Z})$ и $\overline{\mathrm{r}}=(\mathrm{x}, \mathrm{y}, \mathrm{z})$, то

$$
\frac{\mathrm{d} \overline{\mathrm{P}}}{\mathrm{d} \overline{\mathrm{r}}}=\left(\begin{array}{lll}
\frac{\partial \mathrm{X}}{\partial \mathrm{x}} & \frac{\partial \mathrm{Y}}{\partial \mathrm{x}} & \frac{\partial \mathrm{Z}}{\partial \mathrm{x}} \\
\frac{\partial \mathrm{X}}{\partial \mathrm{y}} & \frac{\partial \mathrm{Y}}{\partial \mathrm{y}} & \frac{\partial \mathrm{Z}}{\partial \mathrm{y}} \\
\frac{\partial \mathrm{X}}{\partial \mathrm{z}} & \frac{\partial \mathrm{Y}}{\partial \mathrm{z}} & \frac{\partial \mathrm{Z}}{\partial \mathrm{z}}
\end{array}\right) .
$$

Применяя (5.20) и (5.21), получим 
Ю.И. Блох

$$
\begin{gathered}
\operatorname{grad}_{\mathrm{a}}\left(\overline{\mathrm{I}}(\mathrm{q}) \operatorname{grad}_{\mathrm{a}} \frac{1}{\mathrm{~L}_{\mathrm{qa}}}\right)=\frac{{\mathrm{d} \operatorname{grad}_{\mathrm{a}} \frac{1}{\mathrm{~L}_{\mathrm{qa}}}}_{\mathrm{d} \overline{\mathrm{L}}_{\mathrm{qa}}} \overline{\mathrm{I}}(\mathrm{q})+\frac{\mathrm{d} \overline{\mathrm{I}}(\mathrm{q})}{\mathrm{d} \overline{\mathrm{L}}_{\mathrm{qa}}} \operatorname{grad}_{\mathrm{a}} \frac{1}{\mathrm{~L}_{\mathrm{qa}}}+}{+\left[\overline{\mathrm{I}}(\mathrm{q}) \times \operatorname{rot}_{\operatorname{grad}} \frac{1}{\mathrm{~L}_{\mathrm{qa}}}\right]+\left[\operatorname{grad}_{\mathrm{a}} \frac{1}{\mathrm{~L}_{\mathrm{qa}}} \times \operatorname{rot}_{\mathrm{a}} \overline{\mathrm{I}}(\mathrm{q})\right]} .
\end{gathered}
$$

Поскольку $\operatorname{rot} \operatorname{grad}_{\mathrm{a}} \frac{1}{\mathrm{~L}_{\text {qa }}} \equiv 0$, a $\overline{\mathrm{I}}$ не зависит от координат точки а, (5.22) можно преобразовать:

$$
\operatorname{grad}_{\mathrm{a}}\left(\overline{\mathrm{I}}(\mathrm{q}) \operatorname{grad}_{\mathrm{a}} \frac{1}{\mathrm{~L}_{\mathrm{qa}}}\right)=\mathrm{D}_{3}(\mathrm{q}, \mathrm{a}) \overline{\mathrm{I}}(\mathrm{q}),
$$

где $\mathrm{D}_{3}(\mathrm{q}, \mathrm{a})$ - симметричная матрица третьего порядка, характеризующая в точке а поле, создаваемое шаром с центром в точке q. Ее удобно представить в форме

$$
\mathrm{D}_{3}(\mathrm{q}, \mathrm{a})=\frac{1}{\mathrm{~L}_{\mathrm{qa}}^{3}}\left[\frac{3}{\mathrm{~L}_{\mathrm{qa}}^{2}} \mathrm{~T}_{3}(\mathrm{q}, \mathrm{a})-\mathrm{E}\right] \text {. }
$$

Элементы $\mathrm{t}_{\mathrm{ij}}^{(3)}$ матрицы $\mathrm{T}_{3}(\mathrm{q}, \mathrm{a})$ вычисляются по формуле

$$
\mathrm{t}_{\mathrm{ij}}^{(3)}=\left[\mathrm{x}_{\mathrm{i}}(\mathrm{q})-\mathrm{x}_{\mathrm{i}}(\mathrm{a})\right]\left[\mathrm{x}_{\mathrm{j}}(\mathrm{q})-\mathrm{x}_{\mathrm{j}}(\mathrm{a})\right], \quad \mathrm{i}, \mathrm{j}=1,2,3 .
$$

Учитывая соотношение (5.23), преобразуем уравнение (5.19):

$$
\left[\mathrm{E}+\frac{1}{3} \kappa(\mathrm{a})\right] \overline{\mathrm{I}}(\mathrm{a})=\overline{\mathrm{I}}_{0}(\mathrm{a})+\frac{1}{4 \pi} \kappa(\mathrm{a}) \int_{\mathrm{V}-\mathrm{V}_{\mathrm{a}}} \mathrm{D}_{3}(\mathrm{q}, \mathrm{a}) \overline{\mathrm{I}}(\mathrm{q}) \mathrm{dV} .
$$

Наконец, умножая обе части уравнения (5.26) слева на обратную матрицу $[\mathrm{E}+\kappa(\mathrm{a}) / 3]^{-1}$, получим окончательно

$$
\overline{\mathrm{I}}(\mathrm{a})=\left[\mathrm{E}+\frac{1}{3} \kappa(\mathrm{a})\right]^{-1}\left[\overline{\mathrm{I}}_{0}(\mathrm{a})+\frac{1}{4 \pi} \kappa(\mathrm{a}) \int_{\mathrm{V}-\mathrm{V}_{\mathrm{a}}} \mathrm{D}_{3}(\mathrm{q}, \mathrm{a}) \overline{\mathrm{I}}(\mathrm{q}) \mathrm{dV}\right] .
$$

Таким образом, вместо интегро-дифференциального уравнения (5.6) мы получили интегральное уравнение типа Фредгольма второго рода (5.27), не содержащее сингулярности под интегралом. Для изотропных тел, когда к(а) - скаляр, это уравнение, принимает следующий вид:

$$
\overline{\mathrm{I}}(\mathrm{a})=\frac{3}{3+\kappa(\mathrm{a})}\left[\overline{\mathrm{I}}_{0}(\mathrm{a})+\frac{1}{4 \pi} \kappa(\mathrm{a}) \int_{V-\mathrm{V}_{\mathrm{a}}} \mathrm{D}_{3}(\mathrm{q}, \mathrm{a}) \overline{\mathrm{I}}(\mathrm{q}) \mathrm{dV}\right] .
$$

Для двумерных анизотропных тел аналогичные рассуждения заставляют выделить малый круговой цилиндр с осью, проходящей через точку а, после чего, учитывая, что в системе СИ коэффициент размагничивания кругового цилиндра $\mathrm{N}=0,5$, можно прийти к следующему интегральному уравнению:

$$
\overline{\mathrm{I}}(\mathrm{a})=\left[\mathrm{E}+\frac{1}{2} \kappa(\mathrm{a})\right]^{-1}\left[\overline{\mathrm{I}}_{0}(\mathrm{a})+\frac{1}{4 \pi} \kappa(\mathrm{a}) \int_{\mathrm{S}-\mathrm{S}_{\mathrm{a}}} \mathrm{D}_{2}(\mathrm{q}, \mathrm{a}) \overline{\mathrm{I}}(\mathrm{q}) \mathrm{dS}\right] .
$$

Симметричная матрица второго порядка $\mathrm{D}_{2}(\mathrm{q}, \mathrm{a})$ характеризует в точке а поле, создаваемое круговым цилиндром с осью, проходящей через точку q и выражается в виде:

$$
\mathrm{D}_{2}(\mathrm{q}, \mathrm{a})=\frac{2}{\mathrm{~L}_{\mathrm{qa}}^{2}}\left[\frac{2}{\mathrm{~L}_{\mathrm{qa}}^{2}} \mathrm{~T}_{2}(\mathrm{q}, \mathrm{a})-\mathrm{E}\right]
$$

Соответственно элементы $\mathrm{t}_{\mathrm{ij}}^{(2)}$ матрицы $\mathrm{T}_{2}(\mathrm{q}, \mathrm{a})$ вычисляются по формуле, аналогичной (5.25)

$$
t_{i j}^{(2)}=\left[x_{i}(q)-x_{i}(a)\right]\left[x_{j}(q)-x_{j}(a)\right], \quad i, j=1,2 .
$$


Для изотропных двумерных объектов уравнение (5.28) принимает вид:

$$
\overline{\mathrm{I}}(\mathrm{a})=\frac{2}{2+\kappa(\mathrm{a})}\left[\overline{\mathrm{I}}_{0}(\mathrm{a})+\frac{1}{4 \pi} \kappa(\mathrm{a}) \int_{\mathrm{S}-\mathrm{S}_{\mathrm{a}}} \mathrm{D}_{2}(\mathrm{q}, \mathrm{a}) \overline{\mathrm{I}}(\mathrm{q}) \mathrm{dS}\right] .
$$

Полученные интегральные уравнения лежат в основе алгоритмов решения прямых задач комплексной магниторазведки с учетом размагничивания.

\section{§ 6. Численное решение интегральных уравнений для намагниченности}

Для решения практических задач необходимо иметь эффективный численный метод решения интегральных уравнений. С этой целью изучаемый объект целесообразно аппроксимировать совокупностью одинаковых непересекающихся изометричных элементов достаточно малого размера, чтобы считать каждый из них намагничивающимся однородно как в первичном поле, так и в аномальных полях других элементов. Вообще говоря, изометричность аппроксимирующих элементов не является необходимым условием эффективности алгоритмов решения прямой задачи. В принципе переход от интегродифференциального уравнения к интегральному может осуществляться путем выделения не шара $\mathrm{V}_{\mathrm{a}}$, a, например, эллипсоида. Вводя тензор размагничивания эллипсоида, легко получить интегральное уравнение, аналогичное (5.27), и решать его, аппроксимируя объект многогранниками, описанными вокруг эллипсоида. Этот путь, хотя и может приводить для некоторых частных задач к экономичным алгоритмам, не является технологичным, поскольку аппроксимация произвольного объекта такими многогранниками - не проста. Достаточно сложны и формулы, описывающие аномальное поле произвольного эллипсоида, что и побуждает использовать при аппроксимации изометричные элементы, а их аномальные поля вычислять в трехмерном случае по формулам для вписанного шара, а в двумерном - по формулам для вписанного кругового цилиндра. Именно изометричность элементов позволяет с высокой точностью вычислять поля по простейшим формулам для шаров и цилиндров, существенно экономя машинное время. Одинаковый же их размер дает возможность с наибольшей точностью считать каждый из элементов однородно намагничивающимся в поле других элементов.

При аппроксимации объекта изометричными элементами целесообразно применение правильных многоугольников и многогранников, заполняющих пространство без промежутков. В двумерном случае таковыми являются квадраты и шестиугольники, а в трехмерном - кубы и додекаэдры. Учитывая, что вычисление полей проводится по формулам для вписанных шаров и цилиндров, оптимальными элементами следует признать шестиугольники и додекаэдры, поскольку именно для них вписанные круги и шары создают плотнейшую упаковку [191]. Для них отношение объема элемента к объему вписанного шара или, соответственно, в двумерном случае отношение площадей, меньше, чем для кубов и квадратов. Вместе с тем, аппроксимация кубами или квадратами более технологична, поэтому, несмотря на то, что алгоритмы были реализованы автором в обоих вариантах, при решении практических задач чаще используются именно кубические или квадратные элементы.

Рассмотрим возможность вычисления полей кубических элементов по формулам для вписанного шара, для чего проанализируем поле однородно намагниченного куба, ребро которого равно 2b. Намагниченность куба будем считать вертикальной, что ни в коей мере не ограничивает общности рассмотрения в силу суперпозиции полей, а начало координат поместим в его центр, ориентировав ось Ох параллельно одной из сторон. При $\mathrm{y}=0$ вертикальная компонента Z поля однородно намагниченного куба вычисляется по следующей формуле [109]:

$$
\mathrm{Z}=-2 \mathrm{I}\left\{\operatorname{arctg}\left[\frac{\mathrm{z}+\mathrm{b}}{\mathrm{b}(\mathrm{x}+\mathrm{b})} \sqrt{(\mathrm{x}+\mathrm{b})^{2}+\mathrm{b}^{2}+(\mathrm{z}+\mathrm{b})^{2}}\right]-\operatorname{arctg}\left[\frac{\mathrm{z}-\mathrm{b}}{\mathrm{b}(\mathrm{x}+\mathrm{b})} \sqrt{(\mathrm{x}+\mathrm{b})^{2}+\mathrm{b}^{2}+(\mathrm{z}-\mathrm{b})^{2}}\right]-\right.
$$




$$
\left.-\operatorname{arctg}\left[\frac{\mathrm{z}+\mathrm{b}}{\mathrm{b}(\mathrm{x}-\mathrm{b})} \sqrt{(\mathrm{x}-\mathrm{b})^{2}+\mathrm{b}^{2}+(\mathrm{z}+\mathrm{b})^{2}}\right]+\operatorname{arctg}\left[\frac{\mathrm{z}-\mathrm{b}}{\mathrm{b}(\mathrm{x}-\mathrm{b})} \sqrt{(\mathrm{x}-\mathrm{b})^{2}+\mathrm{b}^{2}+(\mathrm{z}-\mathrm{b})^{2}}\right]\right\} \text {. }
$$

В частности при $\mathrm{x}=0$ :

$$
Z=-4 I\left\{\operatorname{arctg}\left[\frac{z-b}{b^{2}} \sqrt{2 b^{2}+(z-b)^{2}}\right]-\operatorname{arctg}\left[\frac{z+b}{b^{2}} \sqrt{2 b^{2}+(z+b)^{2}}\right]\right\} .
$$

Если кубические элементы, аппроксимирующие объекты, расположены по равномерной сетке, то на наиболее близком расстоянии друг к другу находятся центры соседних элементов, соприкасающихся сторонами, то есть z=2b. При этом

$$
\mathrm{Z}=-4 \mathrm{I}(\operatorname{arctg} \sqrt{3}-\operatorname{arctg} 3 \sqrt{11})=1,693728 \mathrm{I} .
$$

Для шара с учетом поправочного коэффициента, равного отношению объемов куба и шара формула для вычисления компоненты Z при $\mathrm{x}=\mathrm{y}=0$ :

$$
\mathrm{Z}=\frac{16 \mathrm{I} \mathrm{b}^{3}}{\mathrm{Z}^{3}}
$$

При z=2b - Z=2I. Сравнивая это значение с вычисленным по формуле для куба (6.3), видим, что различия составляют 18\%, чем, разумеется, нельзя пренебрегать при вычислениях. Для следующего элемента $\mathrm{z}=4 \mathrm{~b}$, и его поле, как следует из (6.2), равно

$$
\mathrm{Z}=-4 \mathrm{I}(\operatorname{arctg} 3 \sqrt{11}-\operatorname{arctg} 5 \sqrt{27})=0,246784 \mathrm{I} .
$$

Для шара из (6.4) следует Z=0,25 I, и различия в аномалиях не превышают 1,3\%.

На горизонтальной плоскости, проходящей через центр куба, $\mathrm{z}=0$, и формула (6.1) принимает вид:

$$
\mathrm{Z}=-4 \mathrm{I}\left\{\operatorname{arctg}\left[\frac{1}{\mathrm{x}+\mathrm{b}} \sqrt{(\mathrm{x}+\mathrm{b})^{2}+2 \mathrm{~b}^{2}}\right]-\operatorname{arctg}\left[\frac{1}{\mathrm{x}-\mathrm{b}} \sqrt{(\mathrm{x}-\mathrm{b})^{2}+2 \mathrm{~b}^{2}}\right]\right\} .
$$

Для шара с учетом поправки за объем аналогичная формула выглядит так:

$$
|\mathrm{Z}|=\frac{8 \mathrm{I} \mathrm{b}^{3}}{|\mathrm{x}|^{3}} \text {. }
$$

При x=2b для куба

$$
|Z|=-4 I\left(\operatorname{arctg} \frac{\sqrt{11}}{3}-\operatorname{arctg} \sqrt{3}\right)=0,84686 I,
$$

a для эквивалентного шара $|Z|=I$, то есть различия также составляют $18 \%$. На расстоянии $\mathrm{x}=4 \mathrm{~b}$ для куба

$$
|Z|=-4 \mathrm{I}\left(\operatorname{arctg} \frac{\sqrt{27}}{5}-\operatorname{arctg} \frac{\sqrt{11}}{3}\right)=0,123396 \mathrm{I},
$$

а для эквивалентного шара $|\mathrm{Z}|=0,125 \mathrm{I}$, и различие составляет $1,3 \%$.

Для кубов, соприкасающихся ребрами, $\mathrm{x}=\mathrm{z}=2 \mathrm{~b}$. Из (6.1) в этом случае следует

$$
\mathrm{Z}=-2 \mathrm{I}\left(\operatorname{arctg} \sqrt{19}-\operatorname{arctg} \frac{\sqrt{11}}{3}-\operatorname{arctg} 3 \sqrt{11}+\operatorname{arctg} \sqrt{3}\right)=0,172738 \mathrm{I} .
$$

Для эквивалентных шаров

$$
\mathrm{Z}=8 \mathrm{I} \mathrm{b}^{3} \frac{2 \mathrm{z}^{2}-\mathrm{x}^{2}-\mathrm{y}^{2}}{\left(\mathrm{x}^{2}+\mathrm{y}^{2}+\mathrm{z}^{2}\right)^{5 / 2}}
$$

При $\mathrm{y}=0 \quad$ и $\quad \mathrm{x}=\mathrm{z}=2 \mathrm{~b}: \quad \mathrm{Z}=\mathrm{I} /(4 \sqrt{2})=0,176777 \mathrm{I}, \quad$ то есть различия составляют $2,3 \%$. В рассмотренных выше случаях при $\mathrm{x}=0$ или при $\mathrm{z}=0$ горизонтальная компонента аномального поля элемента $\mathrm{H}$, вычисленная и по строгой формуле для куба и по формуле для эквивалентного шара, равна нулю, так что точность ее вычисления оценивать не приходилось. Для кубов, соприкасающихся ребрами, такая оценка необходима. Общая формула для горизонтальной компоненты поля куба при у=0 выглядит следующим образом [109]: 


$$
\begin{gathered}
H=I\left\{\ln \frac{\sqrt{(x+b)^{2}+b^{2}+(z-b)^{2}}-b}{\sqrt{(x+b)^{2}+b^{2}+(z-b)^{2}}+b}-\ln \frac{\sqrt{(x-b)^{2}+b^{2}+(z-b)^{2}}-b}{\sqrt{(x-b)^{2}+b^{2}+(z-b)^{2}}+b}-\right. \\
\left.-\ln \frac{\sqrt{(x+b)^{2}+b^{2}+(z+b)^{2}}-b}{\sqrt{(x+b)^{2}+b^{2}+(z+b)^{2}}+b}+\ln \frac{\sqrt{(x-b)^{2}+b^{2}+(z+b)^{2}}-b}{\sqrt{(x-b)^{2}+b^{2}+(z+b)^{2}}+b}\right\} .
\end{gathered}
$$

При х=z=2b:

$$
\mathrm{H}=\mathrm{I}\left(2 \ln \frac{\sqrt{11}-1}{\sqrt{11}+1}-\ln \frac{\sqrt{3}-1}{\sqrt{3}+1}-\ln \frac{\sqrt{19}-1}{\sqrt{19}+1}\right)=0,53938 \mathrm{I} .
$$

Для эквивалентного шара

$$
H=\frac{24 x z b^{3}}{\left(x^{2}+z^{2}\right)^{5 / 2}}
$$

и в рассматриваемом случае $\mathrm{H}=3 \mathrm{I} /(4 \sqrt{2})=0,53033 \mathrm{I}$, то есть различие составляет $1,7 \%$. Для кубов, соприкасающихся вершинами, различия не превышают 1\%. Таким образом, оценки показывают, что вычисление полей кубических элементов по формулам для вписанного шара с поправкой за объем возможно практически во всех случаях за исключением соседних элементов, соприкасающихся сторонами, когда надо учитывать их фактическую форму и применять формулы (6.5) и (6.9). Для додекаэдров даже в случае соприкасающихся элементов поправки вводить не надо. Как показывает простое сопоставление формул (6.1) с (6.11), а также (6.12) с (6.14), применение этого приема позволяет резко сократить машинное время, затрачиваемое на вычисления. Действительно, в результате его применения время вычислений сокращается практически на порядок.

Перейдем теперь к оценке аналогичной возможности в двумерном случае, когда поля квадратных цилиндров вычисляются по формулам для вписанных круговых цилиндров с поправкой за соотношение их площадей. Соответствующие формулы имеют для квадратного цилиндра следующий вид:

$$
\begin{gathered}
\mathrm{Z}=2 \mathrm{I}\left[\operatorname{arctg} \frac{\mathrm{x}+\mathrm{b}}{\mathrm{z}-\mathrm{b}}-\operatorname{arctg} \frac{\mathrm{x}-\mathrm{b}}{\mathrm{z}-\mathrm{b}}-\operatorname{arctg} \frac{\mathrm{x}+\mathrm{b}}{\mathrm{z}+\mathrm{b}}+\operatorname{arctg} \frac{\mathrm{x}-\mathrm{b}}{\mathrm{z}+\mathrm{b}}\right], \\
\mathrm{H}=\mathrm{I}\left[\ln \frac{(\mathrm{x}+\mathrm{b})^{2}+(\mathrm{z}-\mathrm{b})^{2}}{(\mathrm{x}-\mathrm{b})^{2}+(\mathrm{z}-\mathrm{b})^{2}}-\ln \frac{(\mathrm{x}+\mathrm{b})^{2}+(\mathrm{z}+\mathrm{b})^{2}}{(\mathrm{x}-\mathrm{b})^{2}+(\mathrm{z}+\mathrm{b})^{2}}\right],
\end{gathered}
$$

а для эквивалентного кругового цилиндра

$$
\begin{aligned}
& Z=8 I b^{2} \frac{z^{2}-x^{2}}{\left(x^{2}+z^{2}\right)^{2}}, \\
& H=16 I b^{2} \frac{x z}{\left(x^{2}+z^{2}\right)^{2}} .
\end{aligned}
$$

Для смежных элементов при $\mathrm{x}=0, \mathrm{z}=2 \mathrm{~b}$ - горизонтальная компонента аномального поля $\mathrm{H}=0$, a вертикальная, как следует из (6.15),

$$
\mathrm{Z}=4 \mathrm{I}(\operatorname{arctg} 1-\operatorname{arctg} 1 / 3)=1,854592 \mathrm{I} .
$$

По формуле (6.17) для эквивалентного кругового цилиндра Z=2 I, то есть различие достигает $7,8 \%$. Для следующего из элементов при $\mathrm{x}=0, \mathrm{z}=4 \mathrm{~b}$ соответствующие значения равны

$$
\mathrm{Z}=4 \mathrm{I}(\operatorname{arctg} 1 / 3-\operatorname{arctg} 1 / 5)=0,49742 \mathrm{I} \text {. }
$$

и $Z=0,5 \mathrm{I}$, то есть совпадают с точностью $0,5 \%$. Горизонтальные компоненты в обоих случаях равны нулю. При $\mathrm{z}=0$ и $\mathrm{x}=2 \mathrm{~b}$ или $\mathrm{x}=4 \mathrm{~b}$ аномалии $\mathrm{H}$ также равны нулю, a аномалии $\mathrm{Z}$ отличаются от рассмотренных случаев (6.19) и (6.20) только знаком, так что выводы относительно применимости замены для них аналогичны. Для элементов, соприкасающихся вершинами, при $\mathrm{x}=\mathrm{z}=2 \mathrm{~b}(6.15)$ и (6.17) показывают, что $\mathrm{Z}=0$, а горизонтальная компонента для 
квадратного цилиндра $\mathrm{H}=\mathrm{I} \ln (25 / 9)=1,021651$ I и соответственно для кругового цилиндра $\mathrm{H}=\mathrm{I}$. Различия не превышают 2,2\%.

Таким образом, в двумерном случае выводы, сделанные для трехмерных объектов, остаются в силе: лишь для элементов, соприкасающихся сторонами, расчеты следует вести по формулам (6.19), в остальных случаях точность приближенных вычислений остается практически удовлетворительной, то есть не хуже 2\%. При необходимости особо точных вычислений расчеты по строгим формулам можно вести для несколько более удаленных элементов. Программа, реализующая данный алгоритм, может создать таблицу точных значений компонент аномального поля элементов вплоть до некоторого расстояния, за пределами которого расчет будет проводиться по формулам для эквивалентных шаров или круговых цилиндров. При меньших же расстояниях между элементами в процессе численного решения интегральных уравнений значения полей элементов могут по мере надобности выбираться из этой единожды рассчитанной таблицы, не занимающей в оперативной памяти компьютера большого объема.

При аппроксимации изучаемого объекта совокупностью изометричных элементов обычно приходится решать вопрос о выборе их размеров. Очевидно, чем меньше размеры элементов, тем выше точность расчетов, но больше и затраты машинного времени на их проведение. Приведенные выше оценки полей элементов показывают, что практически целесообразно выбирать их размеры равными примерно одной трети минимального расстояния от объекта до точек, где вычисляется аномальное поле. При больших размерах элементов в аномальных полях может резко проявляться помеха, вызванная тем, что намагниченность каждого из них считается однородной.

При аппроксимации следует соблюдать следующие основные принципы:

1) объемы объекта и аппроксимирующей его конструкции должны быть равными;

2) центры масс объекта и аппроксимирующей его конструкции должны совпадать;

3) аппроксимирующая конструкция должна сохранять угол падения, относительную вытянутость и элементы симметрии объекта.

Соблюдение данных принципов позволяет при сравнительно небольших затратах машинного времени добиться высокой относительной точности вычислений.

Итак, в результате аппроксимации объект заменяется совокупностью М одинаковых изометричных элементов, имеющих объем V. Тогда решение интегрального уравнения (5.27) сводится к решению системы $3 \mathrm{M}$ линейных алгебраических уравнений или $\mathrm{M}$ линейных векторных уравнений. Для k-го элемента соответствующее векторное уравнение запишется в виде

$$
\overline{\mathrm{I}}\left(\mathrm{a}_{\mathrm{k}}\right)=\left[\mathrm{E}+\frac{1}{3} \kappa\left(\mathrm{a}_{\mathrm{k}}\right)\right]^{-1}\left[\overline{\mathrm{I}}_{0}\left(\mathrm{a}_{\mathrm{k}}\right)+\frac{\mathrm{V}}{4 \pi} \kappa\left(\mathrm{a}_{\mathrm{k}}\right) \sum_{\substack{\mathrm{n}=1 \\ \mathrm{n} \neq \mathrm{k}}}^{\mathrm{M}} \mathrm{D}_{3}\left(\mathrm{a}_{\mathrm{n}}, \mathrm{a}_{\mathrm{k}}\right) \overline{\mathrm{I}}\left(\mathrm{a}_{\mathrm{n}}\right)\right] .
$$

Для трехмерных изотропных объектов

$$
\overline{\mathrm{I}}\left(\mathrm{a}_{\mathrm{k}}\right)=\frac{3}{3+\kappa\left(\mathrm{a}_{\mathrm{k}}\right)}\left[\overline{\mathrm{I}}_{0}\left(\mathrm{a}_{\mathrm{k}}\right)+\frac{\mathrm{V}}{4 \pi} \kappa\left(\mathrm{a}_{\mathrm{k}}\right) \sum_{\substack{\mathrm{n}=1 \\ \mathrm{n} \neq \mathrm{k}}}^{\mathrm{M}} \mathrm{D}_{3}\left(\mathrm{a}_{\mathrm{n}}, \mathrm{a}_{\mathrm{k}}\right) \overline{\mathrm{I}}\left(\mathrm{a}_{\mathrm{n}}\right)\right] .
$$

Для двумерных анизотропных объектов при площади поперечного сечения каждого из элементов, равной $\mathrm{S}$, уравнение (5.27) сведется к системе $2 \mathrm{M}$ линейных алгебраических уравнений или М линейных векторных уравнений. Для k-го элемента векторное уравнение имеет следующий вид:

$$
\overline{\mathrm{I}}\left(\mathrm{a}_{\mathrm{k}}\right)=\left[\mathrm{E}+\frac{1}{2} \kappa\left(\mathrm{a}_{\mathrm{k}}\right)\right]^{-1}\left[\overline{\mathrm{I}}_{0}\left(\mathrm{a}_{\mathrm{k}}\right)+\frac{\mathrm{S}}{4 \pi} \kappa\left(\mathrm{a}_{\mathrm{k}}\right) \sum_{\substack{\mathrm{n}=1 \\ \mathrm{n} \neq \mathrm{k}}}^{\mathrm{M}} \mathrm{D}_{2}\left(\mathrm{a}_{\mathrm{n}}, \mathrm{a}_{\mathrm{k}}\right) \overline{\mathrm{I}}\left(\mathrm{a}_{\mathrm{n}}\right)\right] .
$$

Соответственно для двумерных изотропных объектов 


$$
\overline{\mathrm{I}}\left(\mathrm{a}_{\mathrm{k}}\right)=\frac{2}{2+\kappa\left(\mathrm{a}_{\mathrm{k}}\right)}\left[\overline{\mathrm{I}}_{0}\left(\mathrm{a}_{\mathrm{k}}\right)+\frac{\mathrm{S}}{4 \pi} \kappa\left(\mathrm{a}_{\mathrm{k}}\right) \sum_{\substack{\mathrm{n}=1 \\ \mathrm{n} \neq \mathrm{k}}}^{\mathrm{M}} \mathrm{D}_{2}\left(\mathrm{a}_{\mathrm{n}}, \mathrm{a}_{\mathrm{k}}\right) \overline{\mathrm{I}}\left(\mathrm{a}_{\mathrm{n}}\right)\right] \text {. }
$$

Как было отмечено выше, решение систем прямыми методами не экономично, поскольку требует большого объема оперативной памяти применяемого компьютера. В связи с этим более экономично применение метода последовательных приближений. При использовании итерационного подхода необходимо многократно перевычислять матрицы $\mathrm{D}_{3}$ или $\mathrm{D}_{2}$, так что при создании рабочих программ приходится уделять особое внимание проведению этих вычислений предельно экономно. Здесь следует отметить, что в матрице $\mathrm{D}_{3}$ фактически рассчитывается не 9, а только 5 элементов, а в матрице $\mathrm{D}_{2}-$ не 4, а 2. Это связано, во-первых, с тем, что матрицы симметричны, а, во-вторых, с тем, что сумма элементов матриц на главной диагонали равна нулю [39]. К тому же знаменатели в дробях, которыми представляются элементы матриц, все одинаковы. Учет приведенных соображений помогает повышению эффективности алгоритмов.

Решение системы уравнений типа (6.21)-(6.24) ищется в виде следующего ряда:

$$
\overline{\mathrm{I}}\left(\mathrm{a}_{\mathrm{k}}\right)=\overline{\mathrm{I}}_{1}\left(\mathrm{a}_{\mathrm{k}}\right)+\overline{\mathrm{I}}_{2}\left(\mathrm{a}_{\mathrm{k}}\right)+\ldots+\overline{\mathrm{I}}_{\mathrm{m}}\left(\mathrm{a}_{\mathrm{k}}\right)+\ldots,
$$

причем в качестве начального приближения принимается соответственно для уравнения (6.21)

$$
\overline{\mathrm{I}}_{1}\left(\mathrm{a}_{\mathrm{k}}\right)=\left[\mathrm{E}+\frac{1}{3} \kappa\left(\mathrm{a}_{\mathrm{k}}\right)\right]^{-1} \overline{\mathrm{I}}_{0}\left(\mathrm{a}_{\mathrm{k}}\right)
$$

для (6.22)

$$
\overline{\mathrm{I}}_{1}\left(\mathrm{a}_{\mathrm{k}}\right)=\frac{3}{3+\kappa\left(\mathrm{a}_{\mathrm{k}}\right)} \overline{\mathrm{I}}_{0}\left(\mathrm{a}_{\mathrm{k}}\right)
$$

для (6.23)

$$
\overline{\mathrm{I}}_{1}\left(\mathrm{a}_{\mathrm{k}}\right)=\left[\mathrm{E}+\frac{1}{2} \kappa\left(\mathrm{a}_{\mathrm{k}}\right)\right]^{-1} \overline{\mathrm{I}}_{0}\left(\mathrm{a}_{\mathrm{k}}\right),
$$

а для (6.24)

$$
\overline{\mathrm{I}}_{1}\left(\mathrm{a}_{\mathrm{k}}\right)=\frac{2}{2+\kappa\left(\mathrm{a}_{\mathrm{k}}\right)} \overline{\mathrm{I}}_{0}\left(\mathrm{a}_{\mathrm{k}}\right)
$$

При вычислении $\overline{\mathrm{I}}_{0}\left(\mathrm{a}_{\mathrm{k}}\right)$ учитывается неоднородность поля источников в методе искусственного подмагничивания и в низкочастотных индуктивных методах. Последующие члены ряда вычисляются аналогично для всех геофизических методов, указанных выше, по рекуррентным формулам, вытекающим из рассматриваемых уравнений. Для (6.21) формула имеет вид:

$$
\overline{\mathrm{I}}_{\mathrm{m}}\left(\mathrm{a}_{\mathrm{k}}\right)=\mathrm{K}_{3}\left(\mathrm{a}_{\mathrm{k}}\right) \sum_{\substack{\mathrm{n}=1 \\ \mathrm{n} \neq \mathrm{k}}}^{\mathrm{M}} \mathrm{D}_{3}\left(\mathrm{a}_{\mathrm{n}}, \mathrm{a}_{\mathrm{k}}\right) \overline{\mathrm{I}}_{\mathrm{m}-1}\left(\mathrm{a}_{\mathrm{n}}\right)
$$

где $\mathrm{K}_{3}\left(\mathrm{a}_{\mathrm{k}}\right)$ - матрица третьего порядка

$$
\mathrm{K}_{3}\left(\mathrm{a}_{\mathrm{k}}\right)=\frac{\mathrm{V}}{4 \pi}\left[\mathrm{E}+\frac{1}{3} \kappa\left(\mathrm{a}_{\mathrm{k}}\right)\right]^{-1} \kappa\left(\mathrm{a}_{\mathrm{k}}\right) .
$$

Эта матрица для каждого из элементов может быть вычислена однократно. Для изотропных объектов рекуррентная формула имеет такой же вид, как и (6.30), только $\mathrm{K}_{3}\left(\mathrm{a}_{\mathrm{k}}\right)$ в ней рассматривается как число:

$$
\mathrm{K}_{3}\left(\mathrm{a}_{\mathrm{k}}\right)=\frac{3 \kappa\left(\mathrm{a}_{\mathrm{k}}\right) \mathrm{V}}{4 \pi\left[3+\kappa\left(\mathrm{a}_{\mathrm{k}}\right)\right]} .
$$

Для двумерных задач рекуррентная формула имеет вид 
Ю.И. Блох

$$
\overline{\mathrm{I}}_{\mathrm{m}}\left(\mathrm{a}_{\mathrm{k}}\right)=\mathrm{K}_{2}\left(\mathrm{a}_{\mathrm{k}}\right) \sum_{\substack{\mathrm{n}=1 \\ \mathrm{n} \neq \mathrm{k}}}^{\mathrm{M}} \mathrm{D}_{2}\left(\mathrm{a}_{\mathrm{n}}, \mathrm{a}_{\mathrm{k}}\right) \overline{\mathrm{I}}_{\mathrm{m}-1}\left(\mathrm{a}_{\mathrm{n}}\right) .
$$

Для анизотропных объектов $\mathrm{K}_{2}\left(\mathrm{a}_{\mathrm{k}}\right)$ - это матрица второго порядка

$$
\mathrm{K}_{2}\left(\mathrm{a}_{\mathrm{k}}\right)=\frac{\mathrm{S}}{4 \pi}\left[\mathrm{E}+\frac{1}{2} \kappa\left(\mathrm{a}_{\mathrm{k}}\right)\right]^{-1} \kappa\left(\mathrm{a}_{\mathrm{k}}\right),
$$

а для изотропных объектов - число:

$$
\mathrm{K}_{2}\left(\mathrm{a}_{\mathrm{k}}\right)=\frac{\kappa\left(\mathrm{a}_{\mathrm{k}}\right) \mathrm{S}}{2 \pi\left[2+\kappa\left(\mathrm{a}_{\mathrm{k}}\right)\right]} .
$$

Вычисления по рекуррентным формулам прерываются на m-ом приближении при достижении наперед заданной точности:

$$
\max _{\mathrm{k}}\left|\overline{\mathrm{I}}_{\mathrm{m}}\left(\mathrm{a}_{\mathrm{k}}\right)\right|<\varepsilon
$$

\section{§ 7. Процедура учета попарного взаимовлияния элементов. Экспресс-методика}

При проведении расчетов на ЭВМ приходится обрывать итерационный процесс на m-ом приближении, но для учета $(\mathrm{m}+1)$-го и последующих приближений можно применить весьма эффективную процедуру, основанную на физической трактовке описанного алгоритма. Дело в том, что итерационный процесс в данном случае имеет весьма простое физическое истолкование. Если представить каждый объект состоящим из совокупности элементарных частей, то предложенный алгоритм окажется эквивалентным учету взаимовлияния этих частей. Действительно, начальное приближение можно трактовать как вычисление намагниченности каждого из элементов с учетом его собственного размагничивания, но без учета существования других элементов. Рекуррентные формулы, соответственно, могут рассматриваться как вычисление на m-ом этапе намагниченности, индуцированной окружающими элементами, имеющими намагниченности (m-1)-го этапа взаимовлияния. В свете этой трактовки учет $(\mathrm{m}+1)$ го и последующих приближений состоит в учете попарного взаимовлияния элементов c намагниченностями, полученными на m-ом этапе взаимовлияния.

Проиллюстрируем суть процедуры учета попарного взаимовлияния на примере трех элементов. Итерационный алгоритм можно представить в виде графа взаимовлияния (рис. 9). Исходные узлы графа изображают начальные намагниченности. Из узлов исходят линии, характеризующие намагничение других элементов в поле, создаваемом начальной намагниченностью данного элемента. Стандартный граф, иллюстрирующий применение рекуррентных формул (6.30) или (6.33), показан на рис. 9а. Его отличает наличие суммирующих узлов, благодаря которым на любом этапе взаимовлияния вид графа идентичен. Эффективная намагниченность каждого из элементов в соответствии с формулой (6.25) представляет собой сумму намагниченностей во всех относящихся к нему узлах. Если при изображении процесса учета взаимовлияния не пользоваться суммирующими узлами, а рассматривать лишь транзитные узлы, в которые не могут сходиться две и более линий намагничения, но из которых исходят (M-1) линий, где М - число элементов, то число таких узлов должно возрастать от этапа к этапу. Количество исходных узлов равно М, в результате первого этапа взаимовлияния их число становится равным $\mathrm{M}(\mathrm{M}-1)$, после второго $\mathrm{M}(\mathrm{M}-1)^{2}$ и так далее. Эффективная намагниченность элемента и в этом случае определяется как сумма намагниченностей во всех его узлах. Эквивалентный граф с транзитными узлами изображен на рис. 9б. На этом рисунке черными линиями показано формирование той части намагниченности, которая учитывается процедурой попарного взаимовлияния; красные же линии не учитываются ею. Если их вообще не показывать на графе, то он примет вид как на рис. 9в. Очевидно, на всех этапах данной процедуры количество транзитных элементов одинаково и равно М(M-1), а линии, характеризующие намагничение, не разветвляются, что и дает возможность просто описать процедуру. Анализ рис. $9 б$ показывает, что рассматриваемая процедура приближенна, тем не 
Ю.И. Блох

менее, она точно учитывает первый этап взаимовлияния и значительную часть последующих этапов. Именно это определяет ее эффективность при учете $(\mathrm{m}+1)$-го и последующих приближений.

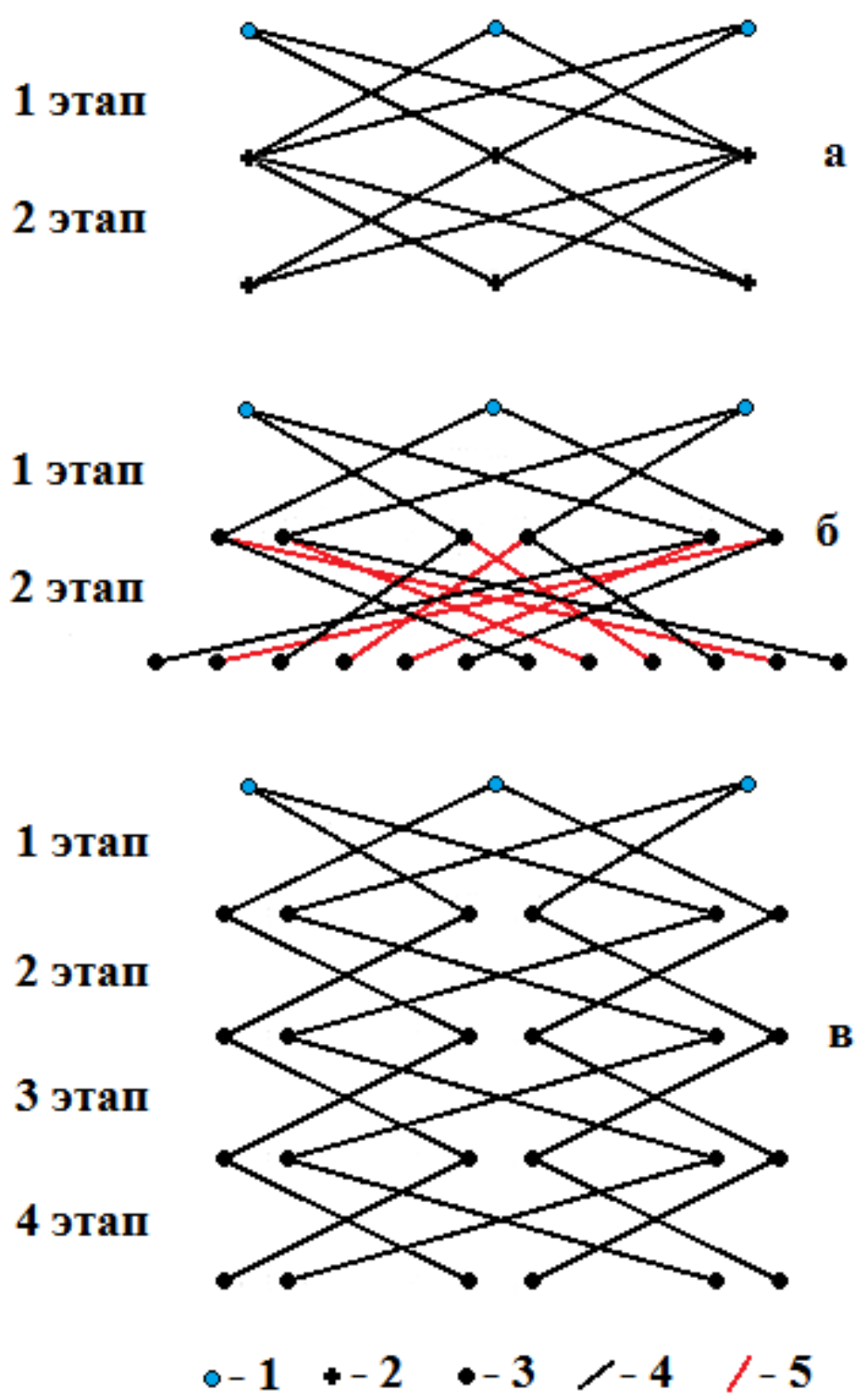

Рис. 9. Графы взаимовлияния трех элементов: а) стандартный с суммирующими узлами,

б) эквивалентный с транзитными узлами, в) попарное взаимовлияние элементов;

1 - исходный узел, 2 - суммирующий узел, 3 - транзитный узел, 4 - намагничение одного из

элементов в поле другого элемента, 5 - намагничение, не учитываемое процедурой попарного взаимовлияния

Перейдем к аналитическому описанию процедуры. Пусть в результате m-ой итерации поправки к намагниченностям k-го и n-го элементов оказались равными $\overline{\mathrm{I}}_{\mathrm{m}}\left(\mathrm{a}_{\mathrm{k}}\right)$ и $\overline{\mathrm{I}}_{\mathrm{m}}\left(\mathrm{a}_{\mathrm{n}}\right)$. Принимая их за начальные намагниченности для двух данных элементов и пренебрегая наличием других элементов, можно записать следующую систему двух векторных уравнений типа (6.21), описывающую взаимовлияние:

$$
\begin{gathered}
\overline{\mathrm{I}}\left(\mathrm{a}_{\mathrm{k}}\right)=\overline{\mathrm{I}}_{\mathrm{m}}\left(\mathrm{a}_{\mathrm{k}}\right)+\mathrm{K}\left(\mathrm{a}_{\mathrm{k}}\right) \mathrm{D}\left(\mathrm{a}_{\mathrm{n}}, \mathrm{a}_{\mathrm{k}}\right) \overline{\mathrm{I}}\left(\mathrm{a}_{\mathrm{n}}\right), \\
\overline{\mathrm{I}}\left(\mathrm{a}_{\mathrm{n}}\right)=\overline{\mathrm{I}}_{\mathrm{m}}\left(\mathrm{a}_{\mathrm{n}}\right)+\mathrm{K}\left(\mathrm{a}_{\mathrm{n}}\right) \mathrm{D}\left(\mathrm{a}_{\mathrm{n}}, \mathrm{a}_{\mathrm{k}}\right) \overline{\mathrm{I}}\left(\mathrm{a}_{\mathrm{k}}\right) .
\end{gathered}
$$


Ю.И. Блох

Теоретические основы комплексной магниторазведки

Отсутствие индексов у матриц K и D вызвано тем, что система аналогична для двумерных и трехмерных моделей. Решение ее получить не сложно. Подставим $\overline{\mathrm{I}}\left(\mathrm{a}_{\mathrm{n}}\right)$ из второго уравнения в первое и перенесем в левую часть члены, содержащие $\overline{\mathrm{I}}\left(\mathrm{a}_{\mathrm{k}}\right)$ :

$$
\left[E-K\left(a_{k}\right) D\left(a_{n}, a_{k}\right) K\left(a_{n}\right) D\left(a_{n}, a_{k}\right)\right] \bar{I}\left(a_{k}\right)=\bar{I}_{m}\left(a_{k}\right)+K\left(a_{k}\right) D\left(a_{n}, a_{k}\right) \bar{I}_{m}\left(a_{n}\right) .
$$

Домножая обе части уравнения (7.2) слева на матрицу, обратную к стоящей в квадратных скобках, получим решение в следующем виде

$$
\overline{\mathrm{I}}\left(\mathrm{a}_{\mathrm{k}}\right)=\left[\mathrm{E}-\mathrm{K}\left(\mathrm{a}_{\mathrm{k}}\right) \mathrm{D}\left(\mathrm{a}_{\mathrm{n}}, \mathrm{a}_{\mathrm{k}}\right) \mathrm{K}\left(\mathrm{a}_{\mathrm{n}}\right) \mathrm{D}\left(\mathrm{a}_{\mathrm{n}}, \mathrm{a}_{\mathrm{k}}\right)\right]^{-1}\left[\overline{\mathrm{I}}_{\mathrm{m}}\left(\mathrm{a}_{\mathrm{k}}\right)+\mathrm{K}\left(\mathrm{a}_{\mathrm{k}}\right) \mathrm{D}\left(\mathrm{a}_{\mathrm{n}}, \mathrm{a}_{\mathrm{k}}\right) \overline{\mathrm{I}}_{\mathrm{m}}\left(\mathrm{a}_{\mathrm{n}}\right)\right] .
$$

Для n-го элемента аналогично

$$
\overline{\mathrm{I}}\left(\mathrm{a}_{\mathrm{n}}\right)=\left[\mathrm{E}-\mathrm{K}\left(\mathrm{a}_{\mathrm{n}}\right) \mathrm{D}\left(\mathrm{a}_{\mathrm{n}}, \mathrm{a}_{\mathrm{k}}\right) \mathrm{K}\left(\mathrm{a}_{\mathrm{k}}\right) \mathrm{D}\left(\mathrm{a}_{\mathrm{n}}, \mathrm{a}_{\mathrm{k}}\right)\right]^{-1}\left[\overline{\mathrm{I}}_{\mathrm{m}}\left(\mathrm{a}_{\mathrm{n}}\right)+\mathrm{K}\left(\mathrm{a}_{\mathrm{n}}\right) \mathrm{D}\left(\mathrm{a}_{\mathrm{n}}, \mathrm{a}_{\mathrm{k}}\right) \overline{\mathrm{I}}_{\mathrm{m}}\left(\mathrm{a}_{\mathrm{k}}\right)\right] .
$$

Несмотря на громоздкость, эти формулы легко реализуются при расчетах на ЭВМ с применением стандартных процедур умножения и обращения матриц.

При использовании выведенных формул в процедуре учета $(\mathrm{m}+1)$-го и последующих приближений требуется вычисление не намагниченностей, а поправок к ним $\Delta \overline{\mathrm{I}}\left(\mathrm{a}_{\mathrm{k}}\right)$, в связи с чем вычислительную формулу для расчета вектора-столбца поправок удобно представить в виде:

$$
\Delta \overline{\mathrm{I}}\left(\mathrm{a}_{\mathrm{k}}\right)=\sum_{\substack{\mathrm{n}=1 \\ \mathrm{n} \neq \mathrm{k}}}^{\mathrm{M}}\left\{\left[\mathrm{A}\left(\mathrm{a}_{\mathrm{n}}, \mathrm{a}_{\mathrm{k}}\right)-\mathrm{E}\right] \overline{\mathrm{I}}_{\mathrm{m}}\left(\mathrm{a}_{\mathrm{k}}\right)+\mathrm{B}\left(\mathrm{a}_{\mathrm{n}}, \mathrm{a}_{\mathrm{k}}\right) \overline{\mathrm{I}}_{\mathrm{m}}\left(\mathrm{a}_{\mathrm{n}}\right)\right\},
$$

где $\mathrm{A}\left(\mathrm{a}_{\mathrm{n}}, \mathrm{a}_{\mathrm{k}}\right)$ и $\mathrm{B}\left(\mathrm{a}_{\mathrm{n}}, \mathrm{a}_{\mathrm{k}}\right)$ - матрицы, вычисляемые в соответствии с (7.3) следующим образом:

$$
\begin{gathered}
A\left(a_{n}, a_{k}\right)=\left[E-K\left(a_{k}\right) D\left(a_{n}, a_{k}\right) K\left(a_{n}\right) D\left(a_{n}, a_{k}\right)\right]^{-1}, \\
B\left(a_{n}, a_{k}\right)=A\left(a_{n}, a_{k}\right) K\left(a_{k}\right) D\left(a_{n}, a_{k}\right) .
\end{gathered}
$$

Отметим, что, вообще говоря, $\mathrm{A}\left(\mathrm{a}_{\mathrm{n}}, \mathrm{a}_{\mathrm{k}}\right) \neq \mathrm{A}\left(\mathrm{a}_{\mathrm{k}}, \mathrm{a}_{\mathrm{n}}\right)$ и $\mathrm{B}\left(\mathrm{a}_{\mathrm{n}}, \mathrm{a}_{\mathrm{k}}\right) \neq \mathrm{B}\left(\mathrm{a}_{\mathrm{k}}, \mathrm{a}_{\mathrm{n}}\right)$, но в случае однородных анизотропных тел, когда тензор магнитной восприимчивости неизменен внутри объекта, эти матрицы оказываются равными.

Для трехмерных изотропных тел могут быть получены явные выражения матриц $\mathrm{A}\left(\mathrm{a}_{\mathrm{n}}, \mathrm{a}_{\mathrm{k}}\right)$ и $\mathrm{B}\left(\mathrm{a}_{\mathrm{n}}, \mathrm{a}_{\mathrm{k}}\right)$, причем для изотропных тел всегда $\mathrm{A}\left(\mathrm{a}_{\mathrm{n}}, \mathrm{a}_{\mathrm{k}}\right)=\mathrm{A}\left(\mathrm{a}_{\mathrm{k}}, \mathrm{a}_{\mathrm{n}}\right)$. Положим

$$
\begin{gathered}
\mathrm{R}^{2}=\sum_{\mathrm{i}=1}^{3}\left[\mathrm{x}_{\mathrm{i}}\left(\mathrm{a}_{\mathrm{n}}\right)-\mathrm{x}_{\mathrm{i}}\left(\mathrm{a}_{\mathrm{k}}\right)\right]^{2}, \\
\mathrm{Q}\left(\mathrm{a}_{\mathrm{k}}\right)=\frac{3 \kappa\left(\mathrm{a}_{\mathrm{k}}\right) \mathrm{V}}{4 \pi \mathrm{R}^{3}\left[3+\kappa\left(\mathrm{a}_{\mathrm{k}}\right)\right]}, \\
\mathrm{Q}\left(\mathrm{a}_{\mathrm{n}}\right)=\frac{3 \kappa\left(\mathrm{a}_{\mathrm{n}}\right) \mathrm{V}}{4 \pi \mathrm{R}^{3}\left[3+\kappa\left(\mathrm{a}_{\mathrm{n}}\right)\right]}, \\
\mathrm{F}_{1}=\frac{1}{1-\mathrm{Q}\left(\mathrm{a}_{\mathrm{k}}\right) \mathrm{Q}\left(\mathrm{a}_{\mathrm{n}}\right)}, \\
\mathrm{F}_{1}=\frac{1}{1-4 \mathrm{Q}\left(\mathrm{a}_{\mathrm{k}}\right) \mathrm{Q}\left(\mathrm{a}_{\mathrm{n}}\right)},
\end{gathered}
$$

тогда

$$
\begin{gathered}
A\left(a_{n}, a_{k}\right)=A\left(a_{k}, a_{n}\right)=\left(F_{2}-F_{1}\right) C_{3}\left(a_{n}, a_{k}\right)+F_{1} E, \\
B\left(a_{n}, a_{k}\right)=Q\left(a_{k}\right)\left[\left(2 F_{2}+F_{1}\right) C_{3}\left(a_{n}, a_{k}\right)-F_{1} E,\right.
\end{gathered}
$$

где элементы $\mathbf{c}_{\mathrm{ij}}^{(3)}$ матрицы $\mathrm{C}_{3}\left(\mathrm{a}_{\mathrm{n}}, \mathrm{a}_{\mathrm{k}}\right)$ определяются по формуле

$$
c_{i j}^{(3)}=\frac{\left[x_{i}\left(a_{n}\right)-x_{i}\left(a_{k}\right)\right]\left[x_{j}\left(a_{n}\right)-x_{j}\left(a_{k}\right)\right]}{\sum_{q=1}^{3}\left[x_{q}\left(a_{n}\right)-x_{q}\left(a_{k}\right)\right]^{2}}, \quad i, j=1,2,3 .
$$


Ю.И. Блох

Теоретические основы комплексной магниторазведки

Если объект однороден, то $\mathrm{B}\left(\mathrm{a}_{\mathrm{n}}, \mathrm{a}_{\mathrm{k}}\right)=\mathrm{B}\left(\mathrm{a}_{\mathrm{k}}, \mathrm{a}_{\mathrm{n}}\right)$, что упрощает вычисления. Отметим также, что для элементов, соприкасающихся гранями, $\mathrm{Q}\left(\mathrm{a}_{\mathrm{k}}\right)$ должны вычисляться по формулам для куба, а не для эквивалентного шара, то есть в соответствии с (6.8)

$$
\mathrm{Q}\left(\mathrm{a}_{\mathrm{k}}\right)=\frac{3 \kappa\left(\mathrm{a}_{\mathrm{k}}\right)}{\pi\left[3+\kappa\left(\mathrm{a}_{\mathrm{k}}\right)\right]}\left(\operatorname{arctg} \sqrt{3}-\operatorname{arctg} \frac{\sqrt{11}}{3}\right)=\frac{0,2021729 \kappa\left(\mathrm{a}_{\mathrm{k}}\right)}{3+\kappa\left(\mathrm{a}_{\mathrm{k}}\right)} .
$$

Для двумерных изотропных объектов также несложно получить явные выражения для элементов матриц $\mathrm{A}\left(\mathrm{a}_{\mathrm{n}}, \mathrm{a}_{\mathrm{k}}\right)$ и $\mathrm{B}\left(\mathrm{a}_{\mathrm{n}}, \mathrm{a}_{\mathrm{k}}\right)$. Если обозначить

$$
\begin{gathered}
\mathrm{R}^{2}=\sum_{\mathrm{i}=1}^{2}\left[\mathrm{x}_{\mathrm{i}}\left(\mathrm{a}_{\mathrm{n}}\right)-\mathrm{x}_{\mathrm{i}}\left(\mathrm{a}_{\mathrm{k}}\right)\right]^{2}, \\
\mathrm{Q}\left(\mathrm{a}_{\mathrm{k}}\right)=\frac{\kappa\left(\mathrm{a}_{\mathrm{k}}\right) \mathrm{S}}{\pi \mathrm{R}^{2}\left[2+\kappa\left(\mathrm{a}_{\mathrm{k}}\right)\right]}, \\
\mathrm{Q}\left(\mathrm{a}_{\mathrm{n}}\right)=\frac{\kappa\left(\mathrm{a}_{\mathrm{n}}\right) \mathrm{S}}{\pi \mathrm{R}^{2}\left[2+\kappa\left(\mathrm{a}_{\mathrm{n}}\right)\right]}, \\
\mathrm{F}=\frac{1}{1-\mathrm{Q}\left(\mathrm{a}_{\mathrm{k}}\right) \mathrm{Q}\left(\mathrm{a}_{\mathrm{n}}\right)},
\end{gathered}
$$

To

$$
\begin{gathered}
A\left(a_{n}, a_{k}\right)=A\left(a_{k}, a_{n}\right)=F E, \\
B\left(a_{n}, a_{k}\right)=F K\left(a_{k}\right) D\left(a_{n}, a_{k}\right)=F Q\left(a_{k}\right)\left[2 C_{2}\left(a_{n}, a_{k}\right)-E\right],
\end{gathered}
$$

где элементы $\mathrm{c}_{\mathrm{ij}}^{(2)}$ матрицы $\mathrm{C}_{2}\left(\mathrm{a}_{\mathrm{n}}, \mathrm{a}_{\mathrm{k}}\right)$ определяются по формуле

$$
\mathrm{c}_{\mathrm{ij}}^{(2)}=\frac{\left[\mathrm{x}_{\mathrm{i}}\left(\mathrm{a}_{\mathrm{n}}\right)-\mathrm{x}_{\mathrm{i}}\left(\mathrm{a}_{\mathrm{k}}\right)\right]\left[\mathrm{x}_{\mathrm{j}}\left(\mathrm{a}_{\mathrm{n}}\right)-\mathrm{x}_{\mathrm{j}}\left(\mathrm{a}_{\mathrm{k}}\right)\right]}{\sum_{\mathrm{q}=1}^{2}\left[\mathrm{x}_{\mathrm{q}}\left(\mathrm{a}_{\mathrm{n}}\right)-\mathrm{x}_{\mathrm{q}}\left(\mathrm{a}_{\mathrm{k}}\right)\right]^{2}}, \quad \mathrm{i}, \mathrm{j}=1,2 .
$$

Для соприкасающихся сторонами элементов в соответствии с (6.19) $\mathrm{Q}\left(\mathrm{a}_{\mathrm{k}}\right)$ должны вычисляться следующим образом:

$$
\mathrm{Q}\left(\mathrm{a}_{\mathrm{k}}\right)=\frac{2 \kappa\left(\mathrm{a}_{\mathrm{k}}\right)}{\pi\left[2+\kappa\left(\mathrm{a}_{\mathrm{k}}\right)\right]}\left(\operatorname{arctg} 1-\operatorname{arctg} \frac{1}{3}\right)=\frac{0,2951674 \kappa\left(\mathrm{a}_{\mathrm{k}}\right)}{2+\kappa\left(\mathrm{a}_{\mathrm{k}}\right)} .
$$

Таким образом, процедура учета $(\mathrm{m}+1)$-го и последующих приближений путем оценки попарного взаимовлияния элементов с намагниченностями, полученными на m-ом этапе, дает возможность вычисления эффективной намагниченности не по формуле (6.25), а по следующей:

$$
\overline{\mathrm{I}}\left(\mathrm{a}_{\mathrm{k}}\right)=\overline{\mathrm{I}}_{1}\left(\mathrm{a}_{\mathrm{k}}\right)+\overline{\mathrm{I}}_{2}\left(\mathrm{a}_{\mathrm{k}}\right)+\ldots+\overline{\mathrm{I}}_{\mathrm{m}}\left(\mathrm{a}_{\mathrm{k}}\right)+\Delta \overline{\mathrm{I}}\left(\mathrm{a}_{\mathrm{k}}\right),
$$

где $\Delta \overline{\mathrm{I}}\left(\mathrm{a}_{\mathrm{k}}\right)$ - поправка, определяемая соотношением (7.5). Применение процедуры учета попарного взаимовлияния значительно повышает экономичность алгоритма решения прямой задачи с учетом размагничивания, поскольку позволяет сократить число итераций, требуемых для достижения заданной точности. Более того, если магнитная восприимчивость изучаемого объекта не очень велика, то данная процедура может быть применена непосредственно после вычисления начального приближения. Это означает, что эффективная намагниченность каждого из элементов в данном случае определяется не по формуле (7.15), а по следующей:

$$
\overline{\mathrm{I}}\left(\mathrm{a}_{\mathrm{k}}\right)=\overline{\mathrm{I}}_{1}\left(\mathrm{a}_{\mathrm{k}}\right)+\Delta \overline{\mathrm{I}}\left(\mathrm{a}_{\mathrm{k}}\right) \text {, }
$$

то есть при полном отсутствии итерационных вычислений по рекуррентным соотношениям. Этот прием является сутью экспресс-метода учета размагничивания, показавшего высокую эффективность. Если магнитная восприимчивость объекта не превышает 1-1,5 ед. СИ, то экспресс-метод позволяет определить его намагниченность с относительной погрешностью, не превышающей нескольких процентов от ее среднего для объекта значения. 
Экспресс-метод будет более подробно оценен в следующих параграфах - здесь же следует отметить, что наиболее эффективно его применение в алгоритмах подбора. Дело в том, что для сложных сильномагнитных объектов невозможно проводить подбор по частям с применением геологического редуцирования в силу взаимовлияния частей [39]. В таких условиях корректировка подбираемой модели, состоящая, например, в добавлении или ликвидации одного элемента, требует пересчета эффективных намагниченностей всех элементов, что поглощает большое количество машинного времени. При использовании экспресс-метода затраты машинного времени значительно сокращаются, поскольку при этом требуется лишь вычисление дополнительных намагниченностей, возникающих в результате попарного взаимовлияния добавленного или ликвидируемого элемента со всеми остальными. Относительное уменьшение машинного времени, необходимого на указанную корректировку модели из М элементов, составляет примерно 0,5М. К примеру, при добавлении к модели, состоящей из 1000 элементов, еще одного элемента, экспресс-метод позволяет добиться сокращения машинного времени, требуемого на пересчет эффективных намагниченностей, более чем в 500 раз. Если исходная модель сложнее, экономия машинного времени, очевидно, еще значительней.

Таким образом, в результате применения предложенных алгоритмов изучаемый объект аппроксимируется совокупностью элементов, каждый из которых имеет свою эффективную намагниченность. Суммируя поля, создаваемые элементами, можно получить аномальное поле всего объекта. Обозначим компоненты вектора индукции аномального магнитного поля объекта $\overline{\mathrm{B}}(\mathrm{a})$ во внешней точке а через X, Y, Z. Тогда в трехмерном случае, вычисляя поля элементов по формулам для эквивалентных шаров, получим

$$
\overline{\mathrm{B}}(\mathrm{a})=\frac{\mu_{0} \mathrm{~V}}{4 \pi} \sum_{\mathrm{k}=1}^{\mathrm{M}} \mathrm{D}_{3}\left(\mathrm{a}, \mathrm{a}_{\mathrm{k}}\right) \overline{\mathrm{I}}\left(\mathrm{a}_{\mathrm{k}}\right) \text {. }
$$

Для двумерных тел

$$
\overline{\mathrm{B}}(\mathrm{a})=\frac{\mu_{0} \mathrm{~S}}{4 \pi} \sum_{\mathrm{k}=1}^{\mathrm{M}} \mathrm{D}_{2}\left(\mathrm{a}, \mathrm{a}_{\mathrm{k}}\right) \overline{\mathrm{I}}\left(\mathrm{a}_{\mathrm{k}}\right),
$$

Аналогично можно вычислять любые функционалы от полученного распределения намагниченности, например, гармонические моменты, интегральные характеристики, градиенты поля, его спектр, автокорреляционную функцию и т.п. Примеры подобных расчетов будут приведены в дальнейшем.

Эффективные намагниченности элементов характеризуют, помимо прочего, аномальные поля внутри изучаемого объекта. Действительно, в центре k-го элемента в соответствии с (5.2):

$$
\overline{\mathrm{I}}\left(\mathrm{a}_{\mathrm{k}}\right)=\kappa\left(\mathrm{a}_{\mathrm{k}}\right)\left[\overline{\mathrm{H}}^{\text {прв }}\left(\mathrm{a}_{\mathrm{k}}\right)+\overline{\mathrm{H}}^{\text {втр }}\left(\mathrm{a}_{\mathrm{k}}\right)\right]+\overline{\mathrm{I}}_{\mathrm{n}}\left(\mathrm{a}_{\mathrm{k}}\right),
$$

тогда для анизотропных объектов

а для изотропных объектов

$$
\overline{\mathrm{H}}^{\text {втр }}\left(\mathrm{a}_{\mathrm{k}}\right)=\kappa^{-1}\left(\mathrm{a}_{\mathrm{k}}\right)\left[\overline{\mathrm{I}}\left(\mathrm{a}_{\mathrm{k}}\right)-\overline{\mathrm{I}}_{\mathrm{n}}\left(\mathrm{a}_{\mathrm{k}}\right)\right]-\overline{\mathrm{H}}^{\text {прв }}\left(\mathrm{a}_{\mathrm{k}}\right),
$$

$$
\overline{\mathrm{H}}^{\text {втр }}\left(\mathrm{a}_{\mathrm{k}}\right)=\frac{1}{\kappa\left(\mathrm{a}_{\mathrm{k}}\right)}\left[\overline{\mathrm{I}}\left(\mathrm{a}_{\mathrm{k}}\right)-\overline{\mathrm{I}}_{\mathrm{n}}\left(\mathrm{a}_{\mathrm{k}}\right)\right]-\overline{\mathrm{H}}^{\text {прв }}\left(\mathrm{a}_{\mathrm{k}}\right) .
$$

При необходимости вычисления внутреннего поля по более густой сети точек, можно прибегнуть к хорошо развитым методам интерполяции, применяя их отдельно к каждой компоненте аномального поля. 


\section{§ 8. Сходимость предложенных алгоритмов}

Как известно, для сходимости любого стационарного итерационного метода, к каковым относятся и предложенные алгоритмы, необходимым и достаточным является условие, накладываемое на величину спектрального радиуса соответствующего оператора шага итерации. Рассмотрим сходимость алгоритма для однородных трехмерных объектов. Так как с ростом магнитной восприимчивости сходимость, очевидно, ухудшается, то из сходимости итерационного процесса для однородной и достаточно большой величины к непосредственно следует сходимость и при меньших ее значениях, в том числе для анизотропных объектов. Для однородных изотропных тел в трехмерном случае оператор шага $\mathrm{W}$ записывается, исходя из (5.28) следующим образом:

$$
W \bar{I}(a)=\frac{3 \kappa}{4 \pi(3+\kappa)} \int_{V-V_{a}} D_{3}(q, a) \bar{I}(q) d V
$$

или в дискретном варианте, исходя из (6.30) и (6.32)

$$
\mathrm{W} \overline{\mathrm{I}}_{\mathrm{m}}\left(\mathrm{a}_{\mathrm{k}}\right)=\frac{3 \kappa \mathrm{V}}{4 \pi(3+\kappa)} \sum_{\substack{\mathrm{n}=1 \\ \mathrm{n} \neq \mathrm{k}}}^{\mathrm{M}} \mathrm{D}_{3}\left(\mathrm{a}_{\mathrm{n}}, \mathrm{a}_{\mathrm{k}}\right) \overline{\mathrm{I}}_{\mathrm{m}}\left(\mathrm{a}_{\mathrm{n}}\right),
$$

Спектральный радиус оператора $\rho(\mathrm{W})$, то есть наибольшее по модулю из его собственных чисел, непосредственно определить сложно, из-за чего обычно пользуются тем фактом, что наибольшее из его собственных чисел не превышает любую из возможных норм оператора. Другое затруднение в данном случае связано с тем, что форма изучаемого объекта может быть различной, и это может приводить к различным способам оценки. Так, исследуя сходимость в аналогичной задаче расчета полей постоянного тока, К.М. Ермохин дополнил изучаемый объект до полного пространства и определил норму оператора, аналогичного (8.1), в гильбертовом пространстве, что привело его к оценке спектрального радиуса [83]. В магнитостатической задаче аналогичная оценка имеет вид

$$
\rho(W) \leq \frac{2 \kappa}{3+\kappa} .
$$

Итерационный процесс сходится тогда, когда $\rho(W)<1$, откуда следует, что сходимость имеет место при к<3 ед. СИ. Вообще говоря, для большинства практических задач такой оценки вполне достаточно, поскольку более высокие средние значения магнитной восприимчивости встречаются у геологических объектов крайне редко. Вместе с тем, существование таких объектов установлено, что побуждает к более детальному анализу данного вопроса.

Возможность усиления оценки (8.3) кроется в наличии при решении рассматриваемой задачи мощнейших компенсационных эффектов. Рассмотрим пространство, заполненное одинаковыми кубами, и вычислим создаваемое им в центре одного из кубов, совпадающих с началом координат, поле. Не уменьшая общности, примем, что исходная намагниченность ориентирована вдоль оси $\mathrm{x}_{3}$. Симметрия задачи подразумевает, что для всякого куба с координатами $\left(\mathrm{x}_{1}, \mathrm{x}_{2}, \mathrm{x}_{3}\right)$ найдутся кубы с центрами в точках $\left(-\mathrm{x}_{1}, \mathrm{x}_{2}, \mathrm{x}_{3}\right)$ и $\left(\mathrm{x}_{1},-\mathrm{x}_{2}, \mathrm{x}_{3}\right)$. Эти кубы создают равные по модулю и противоположно направленные компоненты поля в начале координат, следовательно, и общие горизонтальные компоненты индуцирующего поля всего пространства в начале координат отсутствуют. Покажем, что это справедливо и для вертикальной компоненты. Для нее в частном случае, когда $\mathrm{I}_{1}=\mathrm{I}_{2}=0$, из (5.24) и (5.25) следует

$$
\mathrm{H}_{3}=\mathrm{I}_{3} \mathrm{~V} \frac{2 \mathrm{x}_{3}^{2}-\mathrm{x}_{1}^{2}-\mathrm{x}_{2}^{2}}{4 \pi \mathrm{R}^{5}} .
$$

При $\mathrm{x}_{1}=\mathrm{x}_{2}=\mathrm{x}_{3} \mathrm{H}_{3}=0$, а для любого куба с неравными координатами $\left(\mathrm{x}_{1}, \mathrm{x}_{2}, \mathrm{x}_{3}\right)$ найдутся еще два куба, координаты которых образованы циклической перестановкой, то есть ( $\left.\mathrm{x}_{2}, \mathrm{x}_{3}, \mathrm{x}_{1}\right),\left(\mathrm{x}_{3}, \mathrm{x}_{1}\right.$, $\mathrm{x}_{2}$ ). Для этих трех кубов сумма полей $\mathrm{H}_{3}$ в начале координат, как следует из (8.4), также равна нулю. Рассмотренный компенсационный механизм и лежит в основе того факта, что в полном пространстве размагничивающее поле отсутствует при любых значениях магнитной 
восприимчивости. В свете наличия компенсационного механизма ясно, что оценка (8.3) получилась загрубленной именно в силу того, что норма гильбертова пространства, вычисляемая через скалярное произведение, суммирует все индуцирующие поля с одним знаком. На самом деле для полного пространства алгоритмы сходятся, как видно, и при к>3 ед. СИ.

Рассмотрим такие бесконечные объекты, в которых компенсационных эффектов нет, то есть все индуцирующие поля имеют в начале координат один знак. Именно для таких объектов, как следует из вышеизложенного, сходимость - наихудшая. К этому классу относятся, в частности, в трехмерном случае конусы, а в двумерном - треугольные призмы, расширяющиеся на бесконечность и вытянутые вдоль поля. Элементарные расчеты показывают, что в вершинах аномальное поле таких объектов бесконечно. Это означает, что итерационные алгоритмы для данных объектов будут также приводить к бесконечным компонентам намагниченности и при весьма малых величинах магнитной восприимчивости. Вместе с тем, это не означает расходимости алгоритмов, а отражает лишь физическую реальность, проистекающую из принятой модели намагничения. В природе такие объекты имели бы, естественно, не бесконечную намагниченность, а намагниченность насыщения, но в модели идеализированного ферромагнетика насыщение не принимается во внимание. Из отмеченного факта следует, что для более адекватной оценки спектрального радиуса оператора шага итерации необходимо выбрать такие бесконечные объекты, в которых еще нет бесконечных намагниченностей, но которые близки по форме к конусам и треугольным призмам. В трехмерном случае - это бесконечный квадратный цилиндр, вытянутый вдоль первичного однородного поля, а в двумерном - бесконечный слой, параллельный первичному полю. Для данных объектов аппроксимирующая конструкция представляет собой бесконечную цепочку одинаковых элементов, вытянутую вдоль намагничивающего поля. Очевидно, что намагниченность этих объектов, являющихся частными формами эллипсоидов, I=кH, то есть такая же, как для полного пространства. Это позволяет оценить не только сходимость, но и точность предложенных алгоритмов, что и будет сделано в следующем параграфе.

Выделим в квадратном цилиндре один из элементарных кубов с ребром $2 \mathrm{~b}$ и поместим в его центр начало координат. Поле, создаваемое при равной намагниченности I всеми другими кубами в начале координат, можно представить в виде ряда. Из (8.4) следует, что это поле

$$
\mathrm{H}_{3}=2 \mathrm{IV} \sum_{\mathrm{n}=1}^{\infty} \frac{2}{\mathrm{R}_{\mathrm{n}}^{3}}+\mathrm{H}_{3 \delta},
$$

где $\mathrm{R}_{\mathrm{n}}=2 \mathrm{bn}, \mathrm{a} \mathrm{H}_{3 \delta}$ - поправка, связанная с необходимостью учета поля смежных элементов по формулам для куба, а не для эквивалентного шара. Тогда, учитывая (6.3), получим

$$
\mathrm{H}_{3}=4 \mathrm{I}\left[2(\operatorname{arctg} \sqrt{3}-\operatorname{arctg} 3 \sqrt{11})-1+\sum_{\mathrm{n}=1}^{\infty} \frac{1}{\mathrm{n}^{3}}\right] .
$$

Сумма ряда, вычисленная на компьютере, оказалась равной 1,202044709, откуда $\mathrm{H}_{3}=4,1956348 \cdot \mathrm{I}$, что лишь на $0,16 \%$ отличается от точного значения $4 \pi \mathrm{I} / 3$. Отсюда следует, что в этом крайне неблагоприятном для сходимости случае оператор шага итерации

$$
\mathrm{WI}=\frac{3 \kappa 4,1956348}{4 \pi(3+\kappa)} \mathrm{I}=\frac{1,001634}{3+\kappa} \mathrm{I},
$$

то есть сходимость имеет место, если к $<1836$ ед. СИ.

В двумерном случае с учетом (6.19) аналогично

$$
\mathrm{H}_{3}=4 \mathrm{I}\left[2(\operatorname{arctg} 1-\operatorname{arctg} 1 / 3)-1+\sum_{\mathrm{n}=1}^{\infty} \frac{1}{\mathrm{n}^{2}}\right] .
$$

Сумма ряда в последней формуле равна $\pi^{2} / 6$ [61], откуда $\mathrm{H}_{3}=6,288920 \cdot \mathrm{I}$, что на $0,09 \%$ отличается от точного значения $2 \pi \mathrm{I}$. Отсюда следует, что оператор шага итерации с учетом (6.33) и (6.35) равен 


$$
\mathrm{WI}=\frac{6,288920 \kappa}{2 \pi(2+\kappa)} \mathrm{I}=\frac{1,0009127}{2+\kappa} \mathrm{I},
$$

то есть процесс сходится, если к<2191 ед. СИ.

Таким образом, даже в рассмотренных предельно неблагоприятных для сходимости случаях, итерационный процесс, лежащий в основе предложенных алгоритмов, сходится как геометрическая прогрессия со знаменателем $\rho(\mathrm{W})$, равным в трехмерном случае

$$
\rho(W) \leq \frac{(1+\delta) \kappa_{\max }}{3+\kappa_{\max }},
$$

где $\kappa_{\max }$ - максимальное из значений магнитной восприимчивости модели, а в двумерном случае ( $\delta$ - малая величина):

$$
\rho(\mathrm{W}) \leq \frac{(1+\delta) \kappa_{\max }}{2+\kappa_{\max }} .
$$

Сопоставление последних формул показывает к тому же, что в трехмерном случае скорость сходимости выше, нежели в двумерном.

\section{§ 9. Оценка точности вычислений по предложенным алгоритмам}

Предложенные алгоритмы были реализованы автором в различных модификациях для разных компьютеров, при этом точность вычислений оценивалась несколькими способами. Вопервых, для некоторых однородно намагничивающихся объектов алгоритмы оценивались аналитически. Во-вторых, проводилось сопоставление результатов расчетов по предложенным алгоритмам с аналогичными результатами, полученными другими исследователями по иным алгоритмам. Наконец, в-третьих, для некоторых объектов проводилось сравнение результатов расчетов с экспериментальными данными, полученными физическим моделированием. Рассмотрим некоторые полученные оценки.

Как известно, однородные и изотропные эллипсоиды намагничиваются в однородном поле однородно [135], что относится и к их частным формам, таким как бесконечная пластина или бесконечные цилиндры, намагниченные вдоль оси. В этих случаях размагничивание объектов собственным аномальным полем отсутствует, а их намагниченности определяются по формуле $\mathrm{I}=\kappa \mathrm{H}^{\text {прв }}$. Данные модели дают уникальную возможность оценки точности вычислений по предложенным алгоритмам при бесконечном количестве аппроксимирующих элементов. В предыдущем параграфе были рассчитаны поля, создаваемые элементами, аппроксимирующими пластину и квадратный цилиндр при продольном намагничении. Подставляя (8.8) и (8.6) соответственно в уравнения (6.24) и (6.22), считая намагниченность I постоянной и направленной, как и $\mathrm{H}^{\text {прв }}$, вдоль модели, получим для пластины:

$$
\mathrm{I}=\frac{2}{2+\kappa}\left(\kappa \mathrm{H}^{\text {прв }}+\frac{6,288920 \kappa \mathrm{I}}{4 \pi}\right),
$$

а для квадратного цилиндра

$$
\mathrm{I}=\frac{3}{3+\kappa}\left(\kappa \mathrm{H}^{\text {прв }}+\frac{4,1956348 \kappa \mathrm{I}}{4 \pi}\right)
$$

Решение уравнения (9.1) таково:

$$
\mathrm{I}=\kappa \mathrm{H}^{\text {прв }}+\frac{4 \pi}{4 \pi-0,0057348 \kappa},
$$

а для уравнения (9.2):

$$
\mathrm{I}=\kappa \mathrm{H}^{\text {прв }}+\frac{12 \pi}{12 \pi-0,020534 \kappa} .
$$

Определим значения магнитной восприимчивости, при которых решения отличаются от точных менее чем на 1\%. Оказывается, что для пластины это выполняется при $\kappa<21,7$ ед. СИ, а для 
квадратного цилиндра при к $<18,2$ ед. СИ. Таким образом, во всем диапазоне встречающихся в природе магнитных восприимчивостей горных пород и руд предложенные алгоритмы дают для пластин и цилиндров результаты с относительной погрешностью, не превышающей $1 \%$.

Расчеты по программе, реализующей разработанные алгоритмы для изотропных трехмерных объектов, сопоставлялись с вычислениями, проведенными и опубликованными П. Шармой [251] на основе численного решения интегро-дифференциального уравнения для намагниченности. В качестве модели использовался куб, аппроксимированный 64-мя кубическими элементами и имеющий магнитную восприимчивость 1,26 ед. СИ (0,1 ед. СГС). Остаточная намагниченность считалась отсутствующей, а намагничивающее поле с индукцией 20900 нТл направлялось горизонтально вдоль оси х 1 . Координаты 16-ти верхних элементов в долях половины ребра куба $\mathrm{b}$ и компоненты их эффективной намагниченности, опубликованные П. Шармой [251], сведены в табл. 3. Сопоставление с данными результатами проводилось в различных режимах. В табл. 4 данные П. Шармы приведены совместно с данными, полученными путем итерационных вычислений по рекуррентной формуле (6.30) вплоть до того, когда максимальная из поправок к намагниченностям становилась менее $1 \%$ от первоначальной намагниченности. На это понадобилось 4 итерации. В табл. 4 помещены компоненты эффективной намагниченности без учета и с учетом $(\mathrm{m}+1)$-го и последующих приближений. Сопоставление показывает хорошее совпадение данных, полученных разными способами, и демонстрирует эффективность процедуры учета попарного взаимовлияния элементов. Табл. 5 содержит данные, характеризующие эту процедуру как экспресс-метод учета размагничивания. Сравнение с данными П. Шармы показывает, что для довольно большой магнитной восприимчивости, характеризующей тестовую модель, экспресс-метод дает, тем не менее, среднеквадратическую погрешность определения компонент намагниченности 0,3089 A/м, то есть 2,017\% от средней намагниченности куба, причем время расчетов относительно времени, затраченного итерационным алгоритмом, сократилось примерно в 5 раз.

Таблица 3.

Компоненты эффективной намагниченности куба с к=1,26 ед. СИ (в $\left.1 \cdot 10^{2} \mathrm{~A} / \mathrm{M}\right)$ по данным П. Шармы [251].

\begin{tabular}{|c|c|c|c|c|c|}
\hline \multirow{2}{*}{ №o } & \multicolumn{2}{|c|}{ Координаты центров } & $\mathrm{I}_{1}$ & \multirow{2}{*}{$\mathrm{I}_{2}$} & \multirow{2}{*}{$\mathrm{I}_{3}$} \\
\cline { 2 - 3 } & $\mathrm{X}_{1} / \mathrm{b}$ & $\mathrm{X}_{2} / \mathrm{b}$ & & & 170,6 \\
\hline 1 & $-0,75$ & $-0,75$ & 1509 & 170,6 & 42,4 \\
\hline 2 & $-0,25$ & $-0,75$ & 1626 & 42,4 & $-42,4$ \\
\hline 3 & 0,25 & $-0,75$ & 1626 & $-42,4$ & $-170,6$ \\
\hline 4 & 0,75 & $-0,75$ & 1509 & $-170,6$ & 187,3 \\
\hline 5 & $-0,75$ & $-0,25$ & 1431 & 49,3 & 44,3 \\
\hline 6 & $-0,25$ & $-0,25$ & 1559 & 15,9 & $-44,3$ \\
\hline 7 & 0,25 & $-0,25$ & 1559 & $-15,9$ & $-187,3$ \\
\hline 8 & 0,75 & $-0,25$ & 1431 & $-49,3$ & 187,3 \\
\hline 9 & $-0,75$ & 0,25 & 1431 & $-49,3$ & 44,3 \\
\hline 10 & $-0,25$ & 0,25 & 1559 & $-15,9$ & $-44,3$ \\
\hline 11 & 0,25 & 0,25 & 1559 & 15,9 & $-187,3$ \\
\hline 12 & 0,75 & 0,25 & 1431 & 49,3 & 170,6 \\
\hline 13 & $-0,75$ & 0,75 & 1509 & $-170,6$ & 42,4 \\
\hline 14 & $-0,25$ & 0,75 & 1626 & $-42,4$ & $-42,4$ \\
\hline 15 & 0,25 & 0,75 & 1626 & 42,4 & $-170,6$ \\
\hline 16 & 0,75 & 0,75 & 1509 & 170,6 & \\
\hline
\end{tabular}


Таблица 4.

Компоненты эффективной намагниченности куба с к=1,26 ед. СИ по данным П. Шармы и по результатам вычислений с относительной погрешностью $1 \%$ без учета и с учетом $(\mathrm{m}+1)$-го и последующих приближений.

\begin{tabular}{|c|c|c|c|c|c|c|c|c|c|}
\hline \multirow[t]{2}{*}{ №№ } & \multicolumn{3}{|c|}{ По данным П. Шармы } & \multicolumn{3}{|c|}{$\begin{array}{c}\text { Без учета }(\mathrm{m}+1) \text {-го и } \\
\text { последующих } \\
\text { приближений }\end{array}$} & \multicolumn{3}{|c|}{$\begin{array}{c}\text { С учетом }(\mathrm{m}+1) \text {-го и } \\
\text { последующих } \\
\text { приближений }\end{array}$} \\
\hline & $\mathrm{I}_{1}$ & $\mathrm{I}_{2}$ & $\mathrm{I}_{3}$ & $\mathrm{I}_{1}$ & $\mathrm{I}_{2}$ & $\mathrm{I}_{3}$ & $\mathrm{I}_{1}$ & $\mathrm{I}_{2}$ & $\mathrm{I}_{3}$ \\
\hline 1 & 1509 & 170,6 & 170,6 & 1507,5 & 170,0 & 170,0 & 1509,8 & 169,4 & 169,4 \\
\hline 2 & 1626 & 42,4 & 42,4 & 1627,6 & 42,5 & 42,5 & 1627,2 & 42,4 & 42,4 \\
\hline 3 & 1626 & $-42,4$ & $-42,4$ & 1627,6 & $-42,5$ & $-42,5$ & 1627,2 & $-42,4$ & $-42,4$ \\
\hline 4 & 1509 & $-170,6$ & $-170,6$ & 1507,5 & $-170,0$ & $-170,0$ & 1509,8 & $-169,4$ & $-169,4$ \\
\hline 5 & 1431 & 49,3 & 187,3 & 1428,4 & 48,8 & 187,5 & 1431,0 & 49,4 & 186,0 \\
\hline 6 & 1559 & 15,9 & 44,3 & 1560,4 & 15,6 & 44,7 & 1559,5 & 15,9 & 44,3 \\
\hline 7 & 1559 & $-15,9$ & $-44,3$ & 1560,4 & $-15,6$ & $-44,7$ & 1559,5 & $-15,9$ & $-44,3$ \\
\hline 8 & 1431 & $-49,3$ & $-187,3$ & 1428,4 & $-48,8$ & $-187,5$ & 1431,0 & $-49,4$ & $-186,0$ \\
\hline 9 & 1431 & $-49,3$ & 187,3 & 1428,4 & $-48,8$ & 187,5 & 1431,0 & $-49,4$ & 186,0 \\
\hline 10 & 1559 & $-15,9$ & 44,3 & 1560,4 & $-15,6$ & 44,7 & 1559,5 & $-15,9$ & 44,3 \\
\hline 11 & 1559 & 15,9 & $-44,3$ & 1560,4 & 15,6 & $-44,7$ & 1559,5 & 15,9 & $-44,3$ \\
\hline 12 & 1431 & 49,3 & $-187,3$ & 1428,4 & 48,8 & $-187,5$ & 1431,0 & 49,4 & $-186,0$ \\
\hline 13 & 1509 & $-170,6$ & 170,6 & 1507,5 & $-170,0$ & 170,0 & 1509,8 & $-169,4$ & 169,4 \\
\hline 14 & 1626 & $-42,4$ & 42,4 & 1627,6 & $-42,5$ & 42,5 & 1627,2 & $-42,4$ & 42,4 \\
\hline 15 & 1626 & 42,4 & $-42,4$ & 1627,6 & 42,5 & $-42,5$ & 1627,2 & 42,4 & $-42,4$ \\
\hline 16 & 1509 & 170,6 & $-170,6$ & 1507,5 & 170,0 & $-170,0$ & 1509,8 & 169,4 & $-169,4$ \\
\hline
\end{tabular}

Таблица 5.

Компоненты эффективной намагниченности куба с к=1,26 ед. СИ по данным П. Шармы и по результатам вычислений экспресс-методом.

\begin{tabular}{|c|c|c|c|c|c|c|}
\hline \multirow{2}{*}{ №o } & \multicolumn{3}{|c|}{ По данным П. Шармы } & \multicolumn{2}{c|}{ По расчетам экспресс-методом } \\
\cline { 2 - 7 } & $\mathrm{I}_{1}$ & $\mathrm{I}_{2}$ & $\mathrm{I}_{3}$ & $\mathrm{I}_{1}$ & $\mathrm{I}_{2}$ & $\mathrm{I}_{3}$ \\
\hline 1 & 1509 & 170,6 & 170,6 & 1515 & 176,3 & 176,3 \\
\hline 2 & 1626 & 42,4 & 42,4 & 1692 & 45,3 & 45,3 \\
\hline 3 & 1626 & $-42,4$ & $-42,4$ & 1692 & $-45,3$ & $-45,3$ \\
\hline 4 & 1509 & $-170,6$ & $-170,6$ & 1515 & $-176,3$ & $-176,3$ \\
\hline 5 & 1431 & 49,3 & 187,3 & 1450 & 45,3 & 220,4 \\
\hline 6 & 1559 & 15,9 & 44,3 & 1639 & 15,6 & 45,3 \\
\hline 7 & 1559 & $-15,9$ & $-44,3$ & 1639 & $-15,6$ & $-45,3$ \\
\hline 8 & 1431 & $-49,3$ & $-187,3$ & 1450 & $-45,3$ & $-220,4$ \\
\hline 9 & 1431 & $-49,3$ & 187,3 & 1450 & $-45,3$ & 220,4 \\
\hline 10 & 1559 & $-15,9$ & 44,3 & 1639 & $-15,6$ & 45,3 \\
\hline 11 & 1559 & 15,9 & $-44,3$ & 1639 & 15,6 & $-45,3$ \\
\hline 12 & 1431 & 49,3 & $-187,3$ & 1450 & 45,3 & $-220,4$ \\
\hline 13 & 1509 & $-170,6$ & 170,6 & 1515 & $-176,3$ & 176,3 \\
\hline 14 & 1626 & $-42,4$ & 42,4 & 1692 & $-45,3$ & 45,3 \\
\hline 15 & 1626 & 42,4 & $-42,4$ & 1692 & 45,3 & $-45,3$ \\
\hline 16 & 1509 & 170,6 & $-170,6$ & 1515 & 176,3 & $-176,3$ \\
\hline
\end{tabular}

Необходимо отметить, что влияние размагничивания для рассматриваемого примера весьма ощутимо. Если бы компоненты намагниченности вычислялись без учета размагничивания, они оказались бы следующими: $\mathrm{I}_{1}=2090 \cdot 10^{-2} \mathrm{~A} / \mathrm{M}, \mathrm{I}_{2}=\mathrm{I}_{3}=0$, то есть под 
действием размагничивания намагниченность куба уменьшилась в среднем на $26,7 \%$ и стала заметно неоднородной. Таким образом, оценка показала хорошую сопоставимость результатов с данными, полученными независимым способом.

Для оценки применимости экспресс-метода для анизотропных объектов рассмотрим модель прямоугольного параллелепипеда, аппроксимированного 30 кубическими элементами, как показано на рис. 10. Полосчатость в модели принята горизонтальной, главные компоненты тензора магнитной восприимчивости модели $\kappa_{\mathrm{t}}=1$ ед. СИ, $\kappa_{\mathrm{n}}=0,5$ ед. СИ, остаточная намагниченность считалась отсутствующей. Компоненты вектора индукции однородного намагничивающего поля составляли $\mathrm{B}_{1}=0, \mathrm{~B}_{2}=25000$ нТл, $\mathrm{B}_{3}=43301$ нТл. Составляющие эффективной намагниченности для верхних 15 элементов, полученные разными способами, сведены в табл. 6. Строгий учет размагничивания состоял в расчетах по рекуррентной формуле вплоть до достижения относительной погрешности вычисления составляющих намагниченности, равной $1 \%$ от

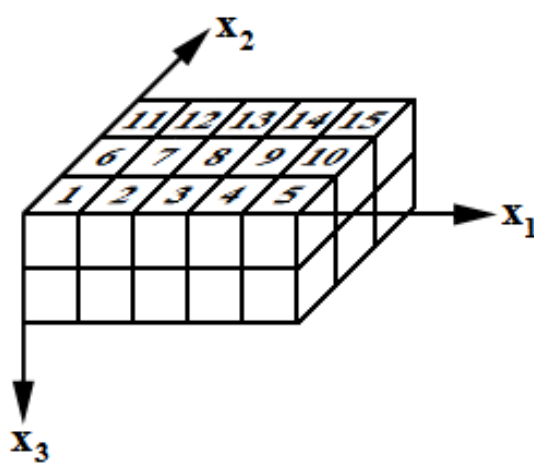

Рис. 10. Аппроксимация прямоугольного параллелепипеда первоначальной, после чего вводилась поправка за $(\mathrm{m}+1)$-е и последующие приближения. Отметим, что без учета размагничивания компоненты эффективной намагниченности были бы одинаковы и составляли: $\mathrm{I}_{1}=0, \mathrm{I}_{2}=19,89 \mathrm{~A} / \mathrm{M}, \mathrm{I}_{3}=17,23 \mathrm{~A} / \mathrm{M}$. Номера элементов в таблице соответствуют рис. 10.

Таблица 6.

Компоненты эффективной намагниченности анизотропного прямоугольного параллелепипеда с

\begin{tabular}{|c|c|c|c|c|c|c|}
\hline \multicolumn{7}{|c|}{$\kappa_{\mathrm{t}}=1$ ед. СИ и $\kappa_{\mathrm{n}}=0,5$ ед. СИ (А/м). } \\
\cline { 2 - 7 } & \multicolumn{7}{|c}{ Эфорективная намагниченность элементов } \\
\cline { 2 - 7 } & Строгий учет размагничивания & \multicolumn{3}{c|}{ Экспресс-метод } \\
\cline { 2 - 7 } & $\mathrm{I}_{1}$ & $\mathrm{I}_{2}$ & $\mathrm{I}_{3}$ & $\mathrm{I}_{1}$ & $\mathrm{I}_{2}$ & $\mathrm{I}_{3}$ \\
\hline 1 & 2,09 & 16,04 & 14,88 & 2,14 & 16,21 & 14,95 \\
\hline 2 & 0,69 & 15,45 & 14,45 & 0,60 & 15,82 & 14,56 \\
\hline 3 & 0 & 15,37 & 14,37 & 0 & 15,74 & 14,48 \\
\hline 4 & $-0,69$ & 15,45 & 14,45 & $-0,60$ & 15,82 & 14,56 \\
\hline 5 & $-2,09$ & 16,04 & 14,88 & $-2,14$ & 16,21 & 14,95 \\
\hline 6 & 0,30 & 14,40 & 13,91 & 0,27 & 14,46 & 13,93 \\
\hline 7 & 0,13 & 13,54 & 13,34 & 0,12 & 13,62 & 13,28 \\
\hline 8 & 0 & 13,40 & 13,23 & 0 & 13,46 & 13,16 \\
\hline 9 & $-0,13$ & 13,54 & 13,34 & $-0,12$ & 13,62 & 13,28 \\
\hline 10 & $-0,30$ & 14,40 & 13,91 & $-0,27$ & 14,46 & 13,93 \\
\hline 11 & 1,01 & 16,19 & 14,01 & 1,12 & 16,71 & 14,05 \\
\hline 12 & 0,32 & 15,46 & 13,43 & 0,28 & 16,10 & 13,43 \\
\hline 13 & 0 & 15,27 & 13,33 & 0 & 15,93 & 13,31 \\
\hline 14 & $-0,32$ & 15,46 & 13,43 & $-0,28$ & 16,10 & 13,43 \\
\hline 15 & $-1,01$ & 16,19 & 14,01 & $-1,12$ & 16,71 & 14,05 \\
\hline
\end{tabular}

Анализ табл. 6 показывает, что при изучении анизотропных объектов экспресс-метод действует также достаточно эффективно. Различия в компонентах намагниченности отдельных элементов при расчетах строгим и экспресс-методом варьируют от 0 до $0,66 \mathrm{~A} / \mathrm{M}$, среднеквадратическое отклонение составляет $0,23 \mathrm{~A} / \mathrm{M}$. Если эти различия оценить относительно модуля средней намагниченности параллелепипеда, составляющей $20,5 \mathrm{~A} / \mathrm{M}$, то можно сделать вывод о том, что погрешность определения отдельных компонент 
намагниченности составляет $1,13 \%$ от модуля средней намагниченности, меняясь для различных элементов от 0 до 3,21\%. Если вычислить аномальное поле непосредственно на поверхности параллелепипеда, то относительная погрешность аномальных значений может достигать при использовании экспресс-метода 3\%, но если в соответствии с основными принципами аппроксимации объектов, изложенных в 3.4, считать, что размеры элемента не превышают $1 / 3$ глубины залегания верхней кромки объекта, то относительная погрешность вычисления аномалий не буде превышать $1,13 \%$.

С целью оценки эффективности предложенных алгоритмов при изучении двумерных моделей, для которых скорость сходимости итерационного процесса ниже, чем для трехмерных, рассматривалась намагниченность однородного изотропного квадратного цилиндра с к=1,26 ед. СИ. Квадратный цилиндр был аппроксимирован 25 элементами, намагничивающее поле с индукцией 50 мкТл считалось вертикальным, а остаточная намагниченность полагалась отсутствующей. Вычисления компонент эффективной намагниченности элементов проводилось в трех режимах, когда процедура учета попарного взаимовлияния применялась после достижения относительной погрешности $0,01 \%, 1 \%$ и сразу после вычисления начального приближения, то есть экспресс-методом. Для достижения точности 0,01\% понадобилось 6 итераций, для 1\% - 3 итерации. Соотношение времени вычислений таким образом, в трех режимах 7:4:1. Результаты вычислений для 9 элементов сведены в табл. 7, для остальных элементов с учетом симметрии задачи они были аналогичны и различались для симметричных элементов лишь знаками горизонтальной компоненты. Координаты центров элементов, нормированные на половину стороны квадрата b и отсчитываемые от его центра, также приведены в табл. 7.

Таблица 7.

Компоненты эффективной намагниченности изотропного квадратного цилиндра с к=1,26 ед.

СИ, рассчитанные в трех режимах.

\begin{tabular}{|c|c|c|c|c|c|c|c|}
\hline \multicolumn{2}{|c|}{ Координаты центров } & \multicolumn{2}{|c|}{$\begin{array}{c}\text { Расчеты с относи- } \\
\text { тельной погреш- } \\
\text { ностью }\end{array}$} & \multicolumn{2}{|c|}{$\begin{array}{c}\text { Расчеты с относи- } \\
\text { тельной погреш- } \\
\text { ностью } 1 \%\end{array}$} & \multicolumn{2}{c|}{$\begin{array}{c}\text { Расчеты экспресс- } \\
\text { методом }\end{array}$} \\
\hline $\mathrm{x}_{1} / \mathrm{b}$ & $\mathrm{x}_{2} / \mathrm{b}$ & $\mathrm{I}_{1}$ & $\mathrm{I}_{2}$ & $\mathrm{I}_{1}$ & $\mathrm{I}_{2}$ & $\mathrm{I}_{1}$ & $\mathrm{I}_{2}$ \\
\hline 0 & 0 & 0 & 30,931 & 0 & 30,932 & 0 & 33,347 \\
\hline 0,4 & 0 & 0 & 31,533 & 0 & 31,534 & 0 & 33,891 \\
\hline 0,8 & 0 & 0 & 33,292 & 0 & 33,289 & 0 & 34,950 \\
\hline 0 & 0,4 & 0 & 30,328 & 0 & 30,327 & 0 & 32,681 \\
\hline 0,4 & 0,4 & 1,204 & 30,952 & 1,206 & 30,952 & 1,217 & 33,231 \\
\hline 0,8 & 0,4 & 2,137 & 32,973 & 2,139 & 32,971 & 2,343 & 34,523 \\
\hline 0 & 0,8 & 0 & 28,522 & 0 & 28,522 & 0 & 30,158 \\
\hline 0,4 & 0,8 & 2,570 & 28,938 & 2,571 & 28,938 & 2,343 & 30,496 \\
\hline 0,8 & 0,8 & 5,812 & 31,425 & 5,814 & 31,426 & 5,729 & 31,904 \\
\hline
\end{tabular}

Анализ таблицы приводит к выводу, что и для двумерных моделей процедура учета попарного взаимовлияния элементов остается весьма эффективной. Ее применение привело к тому, что расчеты с относительной погрешностью $1 \%$ отличаются от более точных расчетов менее чем на $0,1 \%$. Использование ее в качестве экспресс-метода учета размагничивания несмотря на несколько меньшую, нежели для трехмерных моделей, точность, также вполне приемлемо. Среднеквадратическая погрешность определения компонент намагниченности экспресс-методом составила 1,03 А/м, то есть 3,32\% от модуля средней намагниченности. Для отдельных компонент погрешность не превышает 9,5\%. Напомним, что магнитная восприимчивость модели 1,26 ед. СИ была выбрана почти на границе области применимости экспресс-метода, так что полученные погрешности надо рассматривать как предельные. При 
меньших величинах магнитной восприимчивости относительная точность вычислений экспресс-методом возрастает.

Алгоритмы и программы, направленные на изучение изотропных объектов, были разработаны многими исследователями, что позволяет сопоставлять полученные результаты. Для анизотропных объектов такой возможности практически нет, поскольку единственный существующий и имеющий весьма ограниченную применимость алгоритм Л. Эсколы и Т. Терво не содержит в публикации [240] необходимых для этого сведений, поэтому для оценки работоспособности разработанного алгоритма было проведено физическое моделирование [231]. Такую оценку естественно провести для модели, отличающейся значительно большей, нежели у реальных геологических объектов, магнитной восприимчивостью, что связано с ухудшением сходимости алгоритмов при ее росте. Поэтому, если при больших значениях к результаты вычислений достаточно хорошо совпадают с экспериментальными данными, то это гарантирует эффективность алгоритма при меньших величинах $\kappa$.

Физическое моделирование проводилось в переменном, гармонически меняющемся магнитном поле, которое возбуждалось горизонтальной квадратной многовитковой рамкой размерами $1 \times 1 \mathrm{M}$, через которую пропускался ток частотой 80 Гц от генератора звуковых частот. В качестве приемного датчика использовалась катушка длиной 8 мм и такого же диаметра. ЭДС, наведенная в катушке и пропорциональная измеряемому полю, определялась с помощью цифрового вольтметра. Среднеквадратическая погрешность измерений составила $1,5 \%$ от первичного поля рамки.

В качестве модели был использован анизотропный пласт, изготовленный из пластинок трансформаторного железа толщиной 0,35 мм, проложенных листками бумаги толщиной 0,075 мм. Поперечное

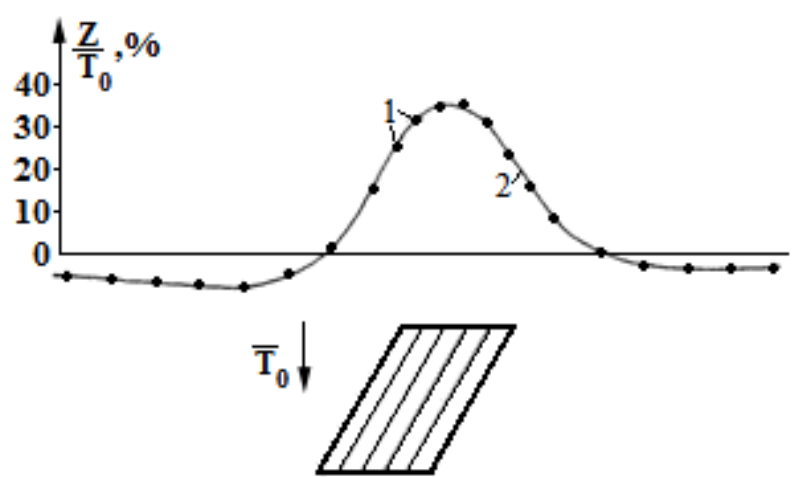

Рис. 11. Сопоставление результатов расчетов с экспериментальными данными для модели пласта с $\kappa_{\mathrm{t}}=641 \mathrm{CИ,} \kappa_{\mathrm{n}}=337$ СИ: 1) график, полученный в результате физического моделирования; 2) график, рассчитанный с помощью предложенного алгоритма сечение пласта показано на рис. 11, длина модели составляла 200 мм. Экспериментально определенные значения магнитной восприимчивости оказались равными $\kappa_{\mathrm{t}}=641$ ед. СИ и $\kappa_{\mathrm{n}}=337$ ед. СИ. Поскольку размеры модели невелики по сравнению с намагничивающей рамкой, а модель располагалась в ее средней трети, намагничивающее поле рамки в объеме модели можно считать практически однородным.

Результаты измерения аномалии вертикальной составляющей в долях первичного поля петли показаны точками на рис. 11. Там же сплошной линией изображен график, рассчитанный с помощью предложенного алгоритма для двумерных тел. Как видно, расхождение не превышает среднеквадратической погрешности физического моделирования. Если проводить расчеты без учета размагничивания, то при данных значениях магнитной восприимчивости результаты будут отличаться от истинных на два порядка.

Таким образом, проведенные различными способами оценки показали достаточно высокую точность вычислений с помощью предложенных алгоритмов. 


\section{ГЛАВА З. ОСНОВНЫЕ ЗАКОНОМЕРНОСТИ НАМАГНИЧЕНИЯ ГЕОЛОГИЧЕСКИХ ОБЪЕКТОВ}

Раздельное дистанционное изучение распределения магнитной восприимчивости и естественной остаточной намагниченности геологических объектов производится в комплексной магниторазведке на основе знаний о том, как намагничиваются эти объекты и как характер их намагничения проявляется в изучаемых полях. Некоторые из необходимых закономерностей могут быть получены аналитически, другие выявляются путем численного моделирования, и в настоящей главе рассматриваются те из них, без которых практически невозможно интерпретировать получаемые в процессе съемок результаты.

\section{§ 10. Намагничение многоугольных цилиндров и многогранников}

Среди интерпретационных моделей, применяемых для аппроксимации геологических объектов, в том числе в комплексной магниторазведке, ведущее место занимают многогранники. Изучению магнитных полей, создаваемых ими, посвящено огромное количество работ. Тем не менее, вплоть до настоящего времени большинство исследователей, придерживающихся традиционной концепции намагничения, пренебрегающей размагничиванием тел в собственном аномальном поле, полагают намагниченность многогранников однородной либо изменяющейся по какому-либо формальному, чаще полиномиальному закону.

Легко показать, что, вообще говоря, неадекватная гипотеза однородного намагничения многогранников ведет к нарушению непрерывности магнитного поля у ребер и вершин. У. Паркинсон в книге «Введение в геомагнетизм» демонстрирует это на простом примере возникновения разрыва поля на ребре пространственного квадранта [142, с. 244-245]. Если в задачах дифракции электромагнитных волн подобное нарушение в принципе допустимо, то в магнитостатике оно противоречит действительности. При этом У. Паркинсон высказал предположение, что непрерывность поля обеспечивается благодаря размагничиванию, в результате которого намагниченность, по его мнению, перераспределяется таким образом, чтобы у ребер она стремилась к нулю [142]. Принципиально правильно поняв роль размагничивания, он, тем не менее, ошибся в конкретных его проявлениях, и это следует исправить.

При исследовании намагничения многогранников важнейшее значение имеют условия сопряжения на границах раздела сред с различными магнитными свойствами, вытекающие из уравнений поля (2.3). В курсах теории поля доказывается, что из уравнения $\operatorname{rot} \overline{\mathrm{H}}=0$ следует непрерывность на гладкой границе тангенциальной компоненты напряженности $\mathrm{H}_{t}$, a из уравнения $\operatorname{div} \overline{\mathrm{B}}=0$ - непрерывность нормальной компоненты индукции $\mathrm{B}_{\mathrm{n}}$ [5]. В областях, границы которых имеют ребра, кромки или вершины, единственности решения прямой задачи можно добиться лишь, сформулировав условия, характеризующие поведение поля у этих особенностей. Обычно таким условием считают требование ограниченности решения в окрестностях особой точки границы.

Рассмотрим вначале двумерную задачу для бесконечного горизонтального многоугольного цилиндра и определим характер магнитного поля у его ребер. Для этого воспользуемся интегральным аналогом первого из дифференциальных уравнений (2.3), то есть $\operatorname{rot} \overline{\mathrm{H}}=0$. Как известно [5], это уравнение выполняется тогда и только тогда, когда циркуляция вектора $\overline{\mathrm{H}}$ по любому плоскому контуру L - нулевая:

$$
\oint_{\mathrm{L}}(\overrightarrow{\mathrm{H}} \mathrm{dl})=0 .
$$


Естественно потребовать, чтобы циркуляция $\overline{\mathrm{H}}$ по любому плоскому контуру, охватывающему ребро и перпендикулярному к нему, была бы нулевой, другими словами, чтобы уравнение $\operatorname{rot} \overline{\mathrm{H}}=0$ выполнялось и у ребра.

Окружим ребро поверхностью кругового цилиндра малого радиуса l (рис. 12a) и вычислим циркуляцию по окружности в плоскости, перпендикулярной ребру. При этом площадку, ограниченную исходным контуром, можно дробить на более мелкие. В соответствии с условием (10.1), по контурам всех площадок циркуляция $\overline{\mathrm{H}}$ также должна быть нулевой. Убирая из исходного круга те его части, которые целиком расположены внутри и вне многоугольного цилиндра и по которым циркуляция, очевидно, нулевая, мы приходим к необходимости анализа циркуляции по многоугольному контуру ABCDEFA (рис. 12б).

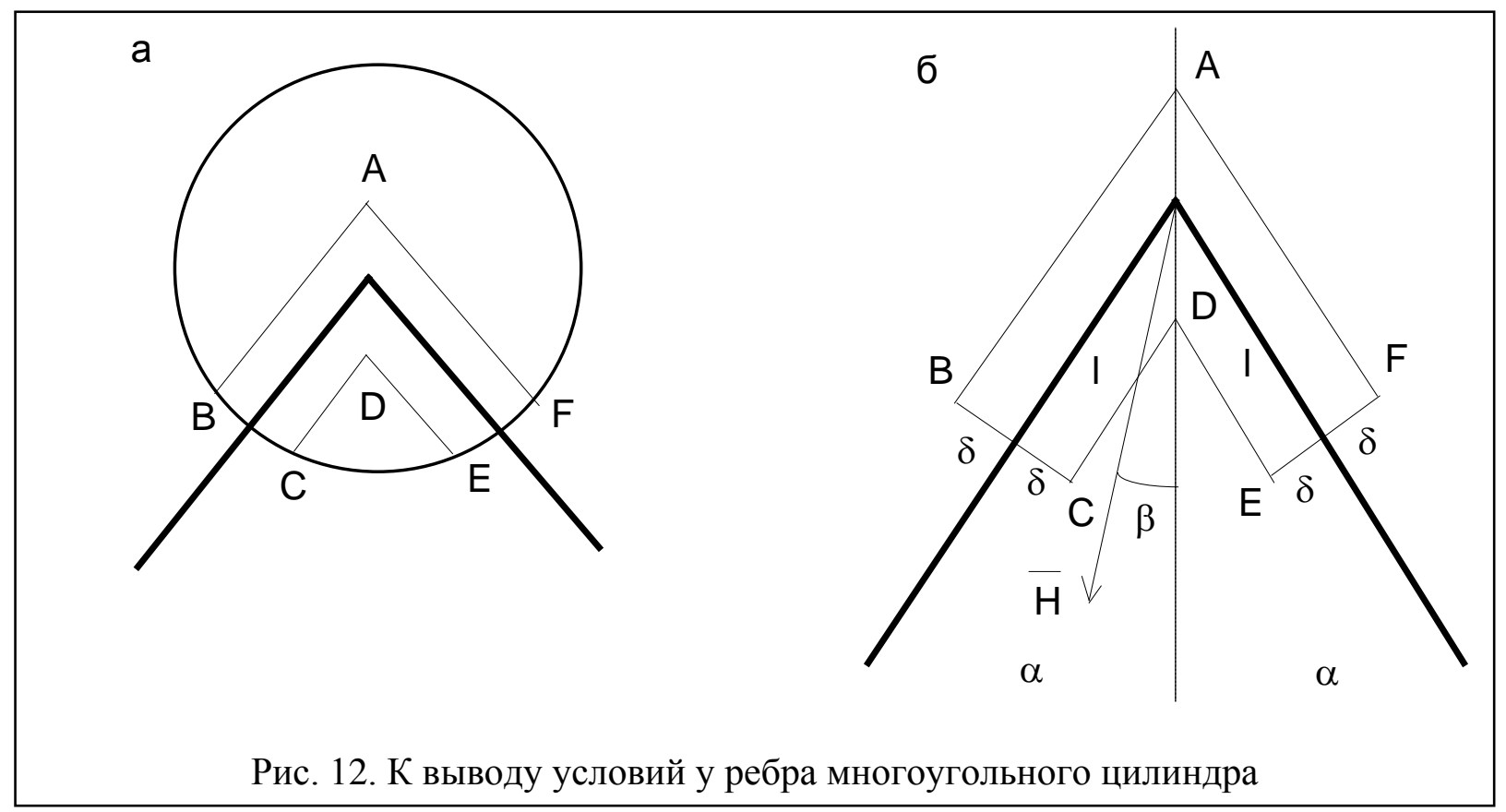

Отрезки $\mathrm{AB}$ и $\mathrm{CD}$ параллельны одной из граней цилиндра и отстоят от нее на малое расстояние $\delta$. Отрезки $\mathrm{AF}$ и $\mathrm{DE}$ расположены на таком же расстоянии от другой грани, примыкающей к данному ребру. Если отрезки ВC и EF - перпендикулярны к соответствующим граням, их вкладом в общую циркуляцию можно пренебречь как малой более высокой степени. Угол при рассматриваемом ребре обозначим $2 \alpha$, а напряженность $\overline{\mathrm{H}}$ магнитного поля в вершине внутри цилиндра будем считать отклоняющейся от биссектральной плоскости на угол $\beta$. Направление обхода примем против часовой стрелки.

Циркуляция по многоугольному контуру ABCDEFA (рис. 12) может быть вычислена как сумма четырех интегралов по отрезкам $\mathrm{AB}, \mathrm{CD}, \mathrm{DE}$ и $\mathrm{FA}$. Поскольку рассматривается малая окрестность ребра, напряженность в этом контуре можно считать постоянной и равной ее значению в вершине. При этом в силу непрерывности $\mathrm{H}_{\mathrm{t}}$ на гранях, интегралы по внешним отрезкам также могут быть выражены через значение Н в вершине. В принятых обозначениях

$$
\begin{gathered}
\int_{\mathrm{AB}}(\mathrm{Hdl})=\mathrm{H} \cos (\alpha-\beta)(\mathrm{l}+\delta \operatorname{ctg} \alpha), \\
\int_{\mathrm{CD}}(\mathrm{Hdl})=-\mathrm{H} \cos (\alpha-\beta)(\mathrm{l}-\delta \operatorname{ctg} \alpha), \\
\int_{\mathrm{DE}}(\mathrm{Hdl})=\mathrm{H} \cos (\alpha+\beta)(\mathrm{l}-\delta \operatorname{ctg} \alpha), \\
\int_{\mathrm{FA}}(\mathrm{Hdl})=-\mathrm{H} \cos (\alpha+\beta)(l+\delta \operatorname{ctg} \alpha)
\end{gathered}
$$


и общая циркуляция окажется равной

$$
\oint(\mathrm{Hdl})=4 \mathrm{H} \delta \cos (\alpha) \sin (\beta) .
$$

Полученное выражение дает возможность анализа характера поля у ребра, для чего надо рассмотреть условия, при которых циркуляция по контуру может быть равна нулю. Очевидно, таких условий - три: либо $\mathrm{H}=0$, либо $\cos \alpha=0$, либо $\sin \beta=0$ [34]. Условие $\cos \alpha=0$ не представляет интереса, поскольку выполняется при развернутом угле $(2 \alpha=\pi)$, когда ребро фактически отсутствует. Условие $\mathrm{H}=0$ соответствует гипотезе У. Паркинсона. Наибольшее же значение имеет условие $\sin \beta=0$. Физически оно означает, что для выполнения уравнения $\operatorname{rot} \overline{\mathrm{H}}=0$ на ребре цилиндра достаточно, чтобы вектор суммарной напряженности был расположен в биссектральной плоскости при этом ребре. Другими словами, для выполнения данного уравнения не надо требовать уменьшения вектора $\mathrm{H}$ на ребре до нуля, как это предлагает У. Паркинсон. Достаточно потребовать уменьшения до нуля под действием размагничивания лишь той компоненты этого вектора, которая перпендикулярна биссектральной плоскости. Именно этот, энергетически более выгодный вариант чаще реализуется в природе, что подтверждают как экспериментальные данные [99-101], так и результаты численного решения интегрального уравнения, в том числе приведенные в предыдушей главе. Уменьшение напряженности в вершине до нуля происходит лишь тогда, когда из-за формы цилиндра и его расположения по отношению к намагничивающему полю не может осуществиться энергетически более выгодный вариант. Примером может служить случай, когда биссектральная плоскость симметричного многоугольного цилиндра перпендикулярна первичному полю. Если у изотропного многоугольного цилиндра отсутствует естественная остаточная намагниченность, то, в соответствии с принятой моделью «идеального ферромагнетика» (1.2), общая намагниченность у ребра также должна быть расположена в биссектральной плоскости.

Перейдем теперь к рассмотрению намагничения произвольных многогранников. Оно определяется характером поведения напряженности магнитного поля у ребер и вершин. Поле у ребер многогранника подчиняется той же закономерности, что и для многоугольных цилиндров. Вблизи вершин могут наблюдаться различные ситуации в зависимости от особенностей данного многогранника. Если существует прямая, проходящая через вершину многогранника и образующая с примыкающими гранями одинаковые углы, то вектор напряженности магнитного поля, как следует из рассмотренного условия у ребер, окажется направленным вдоль нее. Такая прямая, выполняющая роль нормали в вершине, непременно существует, если в данной вершине сходятся три или четыре грани. При большем числе примыкающих граней такой прямой может и не быть. Только в этом случае единственным путем выполнения сформулированного условия оказывается равенство напряженности в данной вершине нулю. В изотропных телах при отсутствии естественной остаточной намагниченности аналогичным закономерностям подчиняется и суммарная намагниченность. В иных случаях направление намагниченности может быть найдено из соотношения (1.2).

Намагниченность симметричных многогранников подчиняется закономерностям, вытекающим из характера поля диполя. Их можно свести к трем основным, указываемым для случая намагничения однородных изотропных тел в однородном магнитном поле при отсутствии естественной остаточной намагниченности.

1. Если многогранник имеет центральную симметрию, то при намагничении в произвольно направленном однородном поле намагниченность в его симметричных точках одинакова как по величине, так и по направлению.

2. Если многогранник имеет плоскость симметрии, параллельную однородному намагничивающему полю, то в его симметричных точках намагниченности окажутся зеркальным отображением друг друга в данной плоскости. Это значит, что компоненты намагниченности, параллельные плоскости симметрии, в этих точках будут одинаковыми, а компоненты, перпендикулярные плоскости симметрии - равными по величине и противоположными по направлению. 
Ю.И. Блох

Теоретические основы комплексной магниторазведки

3. Если многогранник имеет плоскость симметрии, перпендикулярную однородному намагничивающему полю, то в его симметричных точках одинаковыми окажутся компоненты намагниченности, перпендикулярные к плоскости симметрии, а те компоненты, которые параллельны ей, станут равными по величине и противоположными по направлению.

Зная эти закономерности, легко представить качественный характер распределения намагниченности в многоугольных цилиндрах и многогранниках. На рис. 13 показаны распределения намагниченности в некоторых многоугольных цилиндрах с магнитной восприимчивостью 2 СИ, приобретенной в однородном вертикальном поле. Эти примеры получены на основе численного решения интегрального уравнения, рассмотренного в предыдущей главе.
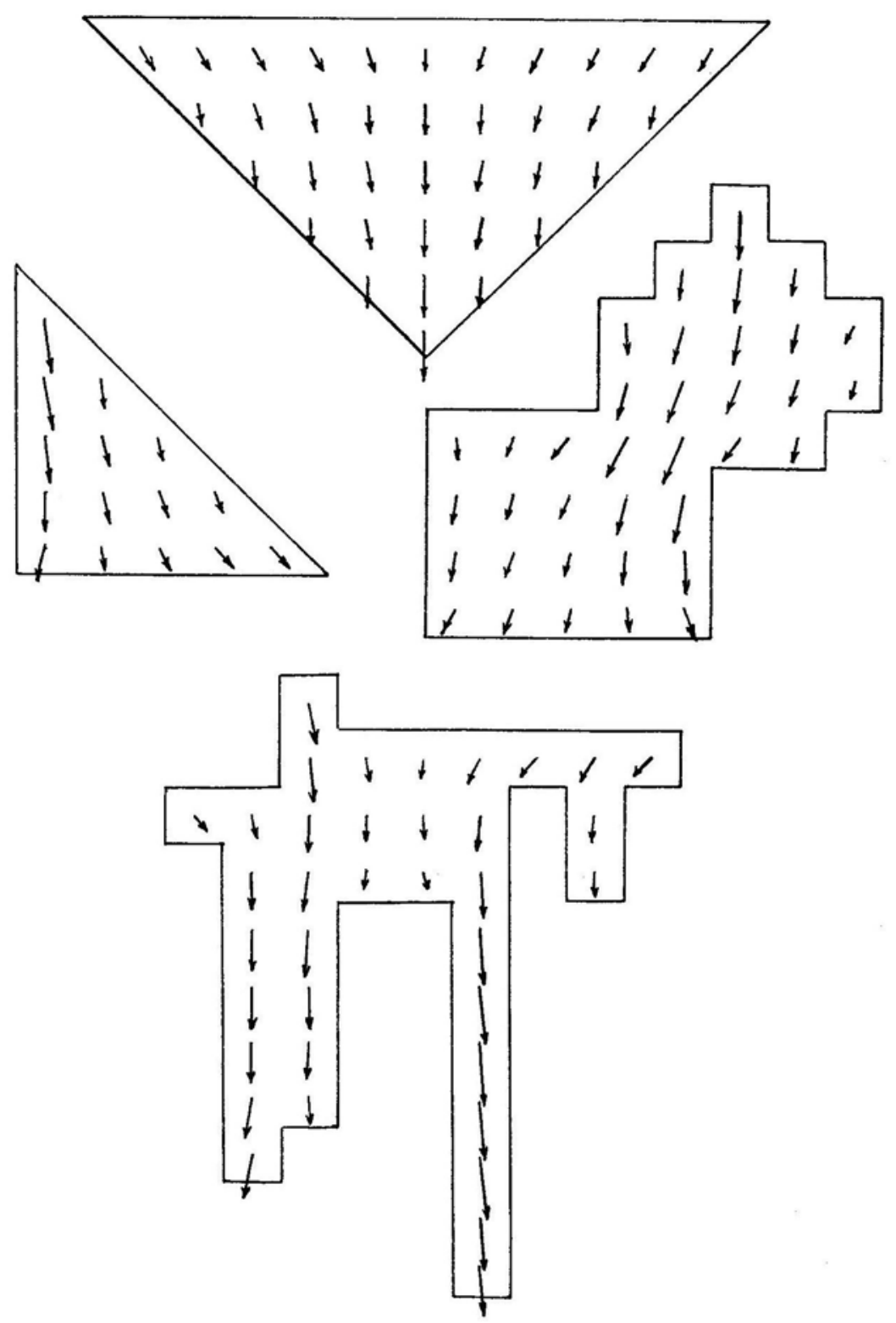

Рис. 13. Распределение намагниченности однородных изотропных многоугольных цилиндров с к=2 СИ, при их намагничении в однородном вертикальном поле

Неоднородность намагничения многогранников под влиянием эффекта размагничивания может даже качественно изменять их аномальное поле. На рис. 14 показаны аномалии вертикальной составляющей индукции магнитного поля $\Delta \mathrm{Z}$ квадратного в сечении цилиндра с 
магнитной восприимчивостью 3 СИ. Намагничивающее поле здесь направлено вертикально вниз и равно 50 мкТл. На рисунке видно, что неоднородность намагничения приводит к тому, что непосредственно над центром куба с высокой магнитной восприимчивостью аномалия вертикальной составляющей имеет не единый максимум, как при однородной намагниченности, а осложняется локальным минимумом и приобретает двугорбую форму. Факты такого рода, очевидно, должны приниматься в расчет при геологическом истолковании.

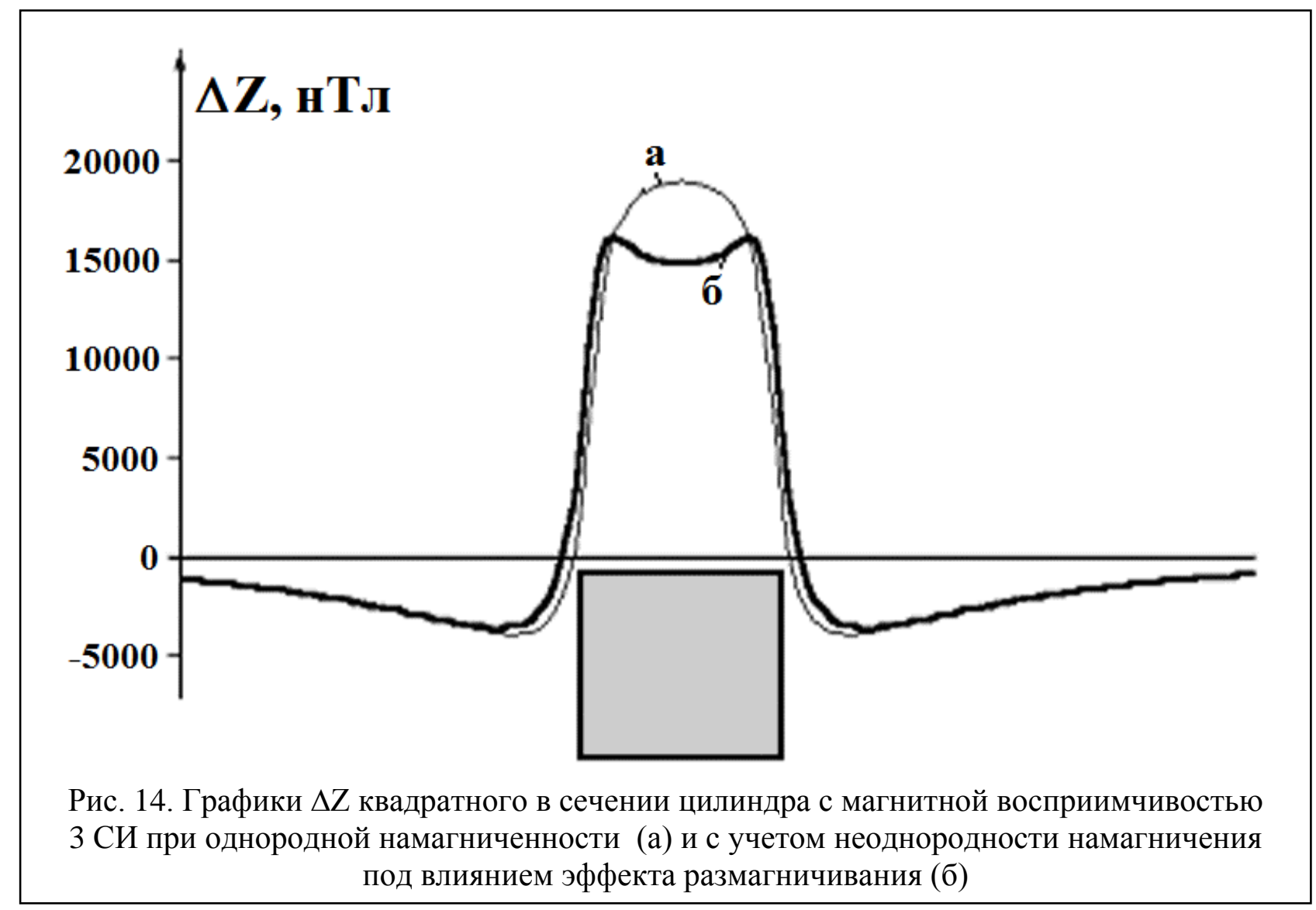

В связи с неоднородностью намагничения многогранников возникает вопрос о том, на каких расстояниях от объекта можно вычислять аномальные поля, пренебрегая ею. Дело в том, что в соответствии с традиционным подходом, восходящим к работам В.КарльгеймГилленшельда начала прошлого века, при вычислении аномалий вдали от сильномагнитного объекта сложной формы его зачастую считают имеющим однородную намагниченность, оцениваемую на эквивалентном эллипсоиде. Магнитные аномалии, связанные с неоднородностью намагничения рассмотренного типа, действительно имеют квадрупольный характер и довольно быстро затухают с удалением от тела. Вместе с тем, пренебрегать влиянием неоднородности намагничения, строго говоря, можно лишь тогда, когда его проявления в аномальном поле не превышают среднеквадратической погрешности проводимой съемки, составляющей в настоящее время не более нескольких нТл.

Как известно, преобразование подобия в магнитных задачах при сохранении магнитных свойств объектов не меняет магнитных аномалий в подобных точках [39]. В силу этого так называемого критерия подобия магнитных полей при оценке проявлений неоднородности намагничения имеют значение не абсолютные удаления от тела, а относительные. Численные эксперименты, проведенные на моделях двумерных и трехмерных тел, показали, что пренебрегать влиянием неоднородности намагничения рассматриваемого типа и использовать традиционные приемы можно лишь тогда, когда расстояние от точки расчета поля до объекта в несколько раз превышает его максимальные линейные размеры. 


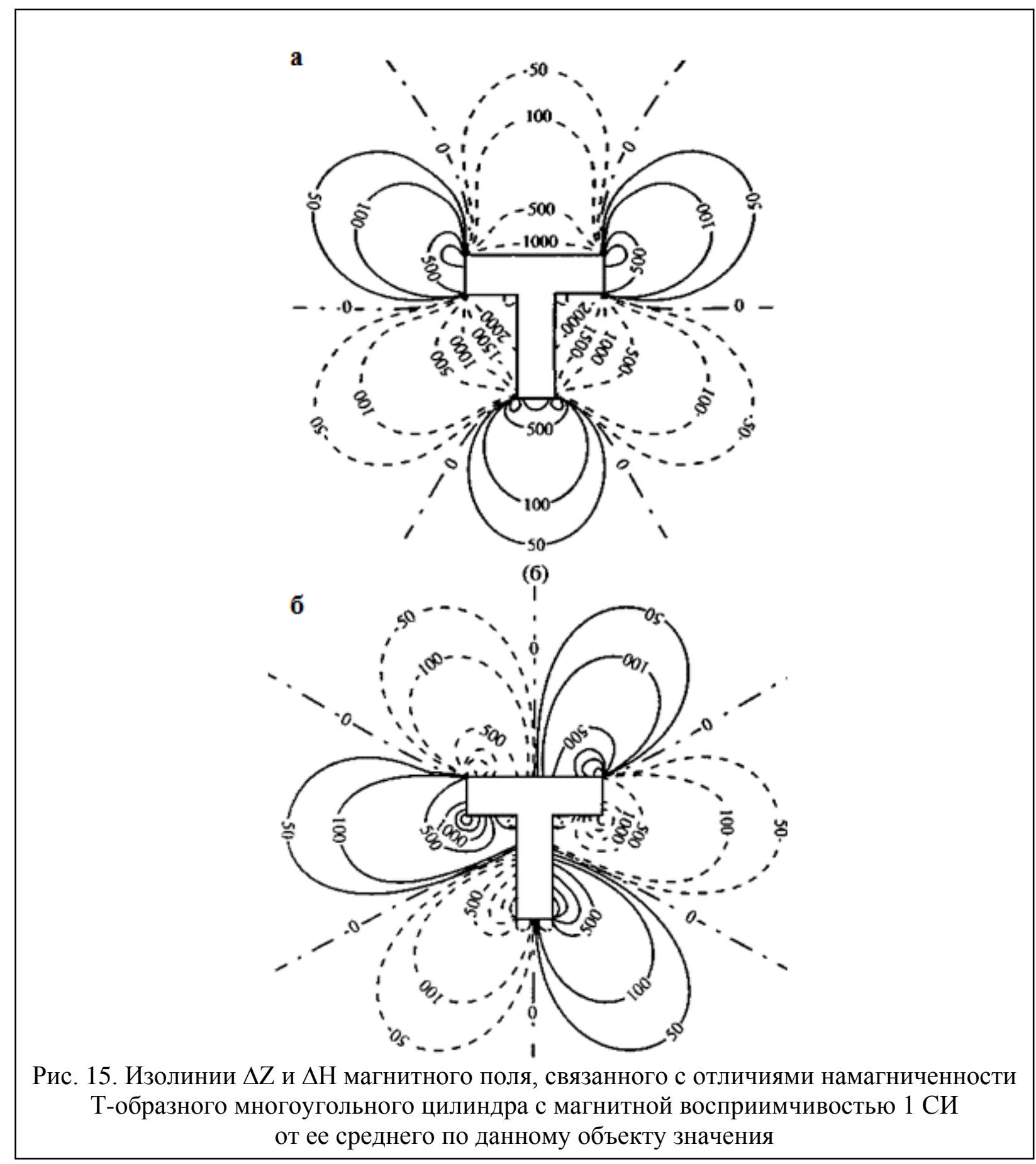

Приведем в качестве иллюстрации один из типичных примеров. На рис. 15 показаны изолинии $\Delta \mathrm{Z}$ и $\Delta \mathrm{H}$ той части магнитного поля, которая связана с отличиями намагниченности Т-образного многоугольного цилиндра от ее среднего по данному объекту значения. При проведении численного эксперимента многоугольник с магнитной восприимчивостью 1 СИ аппроксимировался 57 квадратными элементами; намагничивающее поле считалось вертикальным и равным 50 мкТл. Эффективная намагниченность элементов определялась путем численного решения интегрального уравнения. Средняя намагниченность многоугольника из-за его симметрии оказалась вертикальной и равной $28,782 \mathrm{~A} / \mathrm{M}$, причем вертикальные компоненты намагниченности элементов изменяются от 21,612 до 34,554 A/м, горизонтальные компоненты достигают 4,958 А/м. Для сравнения укажем, что без учета размагничивания намагниченность этого многоугольника была бы однородной, вертикальной и равной 39,789 A/м. Из намагниченности каждого из элементов затем была вычтена средняя намагниченность объекта, поэтому магнитный момент полученного таким образом 
распределения намагниченности оказался равным нулю. Его поле характеризует ту магнитную аномалию, которая связана исключительно с неоднородной частью намагниченности, возникшей в результате размагничивания. Изодинамы вертикальной и горизонтальной составляющих индукции этой части магнитного поля, и представлены на рис. 15. На нем видно, что при удалении от объекта даже на расстояние, равное его максимальному линейному размеру, аномалии от неоднородной части намагниченности превышают 50 нТл. Очевидно, при увеличении магнитной восприимчивости объекта относительные расстояния, на которых необходимо учитывать неоднородность намагничения, связанную с размагничиванием, еще более возрастают.

\section{§ 11. Основные закономерности намагничения пластов в однородных полях}

Наиболее распространенной формой залегания геологических объектов являются пласты, которые характерны и для осадочных, и для интрузивных и для эффузивных пород. Многие виды полезных ископаемых образуют залежи пластовой формы. В связи с этим теория интерпретации магнитных аномалий постоянно обращала пристальное внимание на пласты, но большинство посвященных им работ базируется на традиционной упрощенной концепции намагничения, сложившейся в начале нашего века.

Характерные черты этой устаревшей концепции изложены выше; применительно же к пластам основные еe положения выглядят следующим образом. Если магнитная восприимчивость пласта меньше 0,126 СИ, то его размагничивание не учитывается вообще. При к>0,126 СИ размагничивание учитывается приближенно на вписанном в пласт эллипсоиде, так что даже при больших значениях магнитной восприимчивости он считается однородно намагничивающимся. Среди пластовых моделей при интерпретации наиболее часто применяют модель полубесконечного пласта - такого, у которого нижняя кромка удалена на бесконечно большое расстояние. Выбором такой модели обычно хотят подчеркнуть, что нижняя кромка удалена настолько глубоко, что в пределах площади съемки не оказывает заметного влияния на аномалии. Для полубесконечных пластов намагниченность оценивают по формулам для бесконечного плоскопараллельного слоя - вырожденного эллиптического цилиндра. У слоя при намагничении в продольном однородном поле с напряженностью $\mathrm{H}_{\mathrm{tan}}^{\text {прв }}$ размагничивание отсутствует: $\mathrm{N}=0$. Если слой имеет продольную естественную остаточную намагниченность $\mathrm{I}_{\text {ntan, }}$ то его общая намагниченность

$$
\mathrm{I}_{\mathrm{tan}}=\kappa \mathrm{H}_{\tan }^{\mathrm{np \beta}}+\mathrm{I}_{\mathrm{ntan}} \cdot
$$

При намагничении слоя в поперечном поле $\mathrm{H}_{\text {nог }}^{\text {прв }}$ размагничивание проявляется максимально: $\mathrm{N}=1$, тогда при наличии естественной остаточной намагниченности $\mathrm{I}_{\text {nnor }}$ его общая намагниченность вычисляется по формуле:

$$
I_{\text {nor }}=\frac{\kappa H_{\text {nor }}^{\text {пp }}+I_{\text {nnor }}}{1+\kappa} \text {. }
$$

Обратим внимание на то, что размагничиванию подвергается и остаточная намагниченность.

При намагничении бесконечного слоя в поле, направленном под острым углом $\varphi$ к нему, применяя принцип суперпозиции полей, можно установить, комбинируя (11.1) и (11.2), что вектор намагниченности отклоняется от направления намагничивающего поля в сторону слоя. Если считать $\mathrm{I}_{\mathrm{ntan}}=\mathrm{I}_{\text {nnor }}=0$, а угол между вектором намагниченности и слоем обозначить $\psi$, то

$$
\operatorname{tg} \psi=\frac{1}{1+\kappa} \operatorname{tg} \varphi
$$

Изложенное и составляет традиционную концепцию намагничения пластов, которая лежит в основе существующих методов интерпретации магнитных аномалий.

Даже при поверхностном анализе нетрудно заметить неправомерность замены полубесконечных объектов бесконечными. Не удивительно, что традиционная концепция вступает в противоречие с экспериментальными результатами. Отдельные частные случаи 
подвергали анализу Е.П. Мохова [134], А.А. Попов [146], В.В. Филатов [200], Ю.В. Антонов и С.В. Слюсарев [11], В.М. Девицын, М.И. Лапина и Б.Л. Шнеерсон [76], П. Шарма [251], и все исследователи убеждались, что пласты в однородном поле намагничиваются неоднородно.

Важнейшее значение имеет давно известный и противоречащий традиционной концепции намагничения факт, свидетельствующий о том, что полупространство в нормальном к нему поле размагничивается как круговой цилиндр, то есть имеет коэффициент размагничивания $\mathrm{N}=0,5$ [39]. Его важность для рассматриваемого вопроса состоит в том, что полупространство это предельно мощный пласт. В связи с этим закономерности, справедливые для полупространства, должны соблюдаться и в середине верхней кромки вертикального пласта конечной мощности. Рассмотрим кратко доказательство этого принципиально важного факта для случая, когда полупространство имеет не только магнитную восприимчивость к, но и нормальную к его поверхности естественную остаточную намагниченность $\mathrm{I}_{\mathrm{n}}$. Если напряженность нормального намагничивающего поля $\mathrm{H}^{\text {прв }}$, то, учитывая, что внешнее аномальное поле однородно намагниченного полупространства вычисляется в системе СИ по формуле [39]

$$
\mathrm{Z}=\frac{\mu_{0} \mathrm{I}}{2}
$$

получим для нормальной компоненты напряженности внешнего поля

$$
\mathrm{H}_{\text {nor }}^{\mathrm{e}}=\mathrm{H}^{\text {прв }}+0,5 \mathrm{I} \text {. }
$$

Для внутреннего поля

$$
\mathrm{H}_{\text {nor }}^{\mathrm{i}}=\mathrm{H}^{\text {прв }}-0,5 \mathrm{I} \text {. }
$$

С учетом остаточной намагниченности индукцию вне полупространства и внутри него можно записать как

$$
\begin{gathered}
\mathrm{B}_{\text {nor }}^{\mathrm{e}}=\mu_{0}\left(\mathrm{H}^{\text {прв }}+0,5 \mathrm{I}\right), \\
\mathrm{B}_{\text {nor }}^{\mathrm{i}}=\mu_{0} \mu\left(\mathrm{H}^{\text {прв }}-0,5 \mathrm{I}\right)+\mu_{0} \mathrm{I}_{\mathrm{n}} .
\end{gathered}
$$

Принимая во внимание, что в системе СИ относительная магнитная проницаемость $\mu=1+\kappa$, получим из условия непрерывности $\mathrm{B}_{\text {nоr }}$ на границе

$$
\mathrm{I}=\frac{\kappa \mathrm{H}^{\text {прв }}+\mathrm{I}_{\mathrm{n}}}{1+0,5 \kappa},
$$

то есть $\mathrm{N}=0,5$, что и требовалось доказать.

Таким образом, традиционная концепция намагничения пластов нуждается в серьезной коррекции. При этом, конечно, следует учитывать, что пласты - это частные случаи многоугольных цилиндров и на них распространяются закономерности, установленные в предыдущем параграфе. Здесь же мы, понимая, каков характер намагничения пластов у вершин их кромки, сосредоточимся на анализе распределения их намагниченности в плоскости их симметрии, другими словами, вдоль пластов. Все возможные случаи намагничения данной модели с помощью принципа суперпозиции полей могут быть сведены к двум основным ситуациям, когда пласт намагничен вдоль и поперек, причем достаточно при этом пласт считать изотропным. Действительно, при намагничении анизотропного пласта в продольном однородном поле распределение его намагниченности в плоскости симметрии характеризуется в основном величиной $\kappa_{\mathrm{t}}$, а при намагничении в поперечном поле $-\kappa_{\mathrm{n}}$. Отсюда следует, что результаты изучения намагниченности тонкого изотропного пласта в двух основных ситуациях могут быть с успехом распространены и на анизотропные пласты.

Итак, пусть однородный пласт расположен вертикально и намагничивается в продольном ему однородном вертикальном поле. Величины намагниченности в центре верхней кромки и на асимптоте при удалении на глубину могут быть элементарно вычислены, исходя из принципа подобия магнитных полей. Как отмечалось выше, в середине верхней кромки вертикального пласта конечной мощности его намагниченность должна быть равна намагниченности 
полупространства с такими же магнитными свойствами. Если в соответствии с (3.20) формула для расчета намагниченности однородно намагничивающихся тел имеет вид

$$
\mathrm{I}=\frac{\kappa \mathrm{H}^{\mathrm{np \beta}}+\mathrm{I}_{\mathrm{n}}}{1+\kappa \mathrm{N}},
$$

то из (11.8) следует, что у верхней кромки намагниченность вычисляется по этой формуле при $\mathrm{N}=0$,5. По мере удаления от верхней кромки намагниченность монотонно возрастает и на бесконечности асимптотически стремится к той, какая была бы у бесконечного, а не полубесконечного объекта, то есть соответствующей $\mathrm{N}=0$ в формуле (11.9).

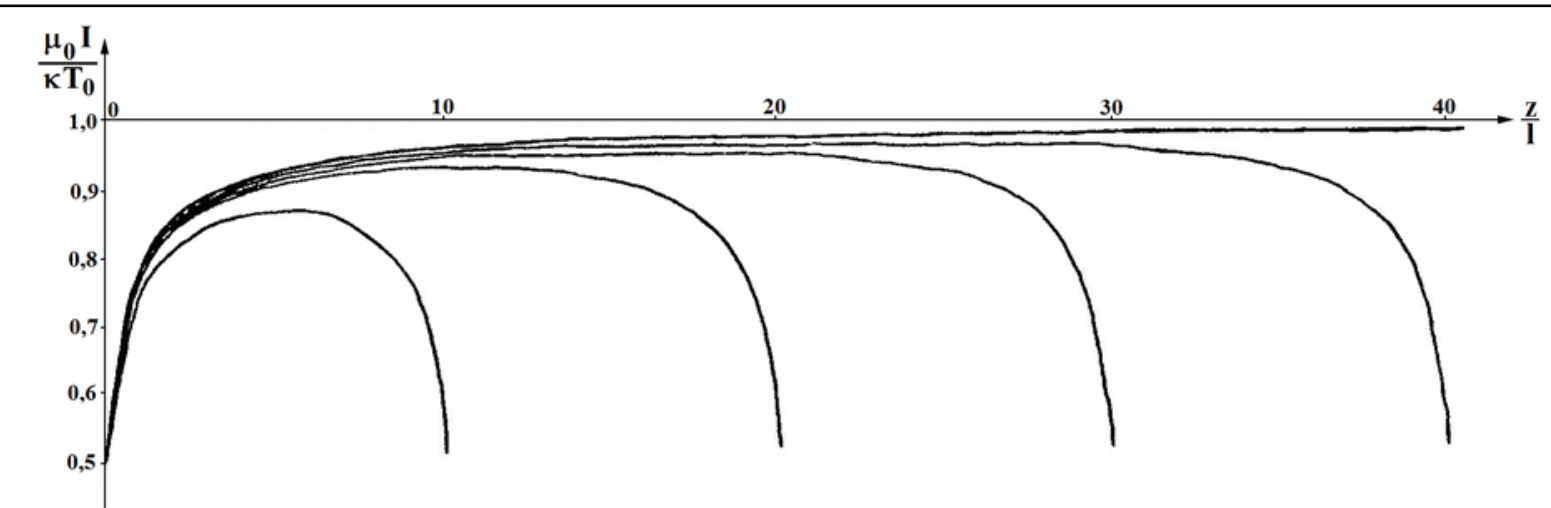

Рис. 16. Распределение намагниченности в тонких пластах с к=2 СИ при различных вытянутостях их поперечного сечения

Чтобы понять характер изменения намагниченности вдоль продольно намагничиваемого пласта, можно прибегнуть к численному моделированию с помощью описанного в предыдущей главе алгоритма, но на компьютере можно рассчитывать намагниченность лишь конечных элементов, поэтому моделирование здесь несколько сложнее, нежели для простых моделей. На рис. 16 показаны распределения намагниченности для конечных тонких пластов с магнитной восприимчивостью 2 СИ при разных вытянутостях их поперечного сечения. Рассчитанная намагниченность каждого из элементов модели I нормирована на намагниченность, полученную в рамках традиционной концепции, $\kappa \mathrm{T}_{0} / \mu_{0}$, то есть без учета размагничивания при $\mathrm{N}=0$. Анализ графиков показывает, что при больших значениях к намагниченность пласта неоднородна. Размагничивание в рассматриваемой ситуации приводит к тому, что верхняя и нижняя кромки пласта намагничиваются значительно слабее, нежели его центр. Намагниченность в центре пласта зависит от его относительной вытянутости, причем лишь когда длина пласта в 100 и более раз превышает его мощность, можно считать, что размагничивающее поле в центре практически отсутствует. Дальнейшее увеличение вытянутости пласта практически не оказывает влияния на характер его намагниченности у верхней и нижней кромок, что и дает возможность распространить полученные результаты на полубесконечные пласты.

На рис. 17а показаны соответствующие графики для полубесконечных пластов с различными магнитными восприимчивостями. При получении данных графиков они были достроены до верхней кромки на основании формулы (11.8) для полупространства. С помощью полученной палетки $\mathrm{K}_{\mathrm{t}}$ для любого пласта, намагниченного в продольном поле, можно найти его намагниченность в центральной плоскости по формуле:

$$
\mathrm{I}=\mathrm{K}_{\mathrm{t}} \frac{\kappa \mathrm{T}_{0}}{\mu_{0}} .
$$

При намагничении пластов в однородном поперечном поле характер влияния размагничивания иной. Максимально оно в центре пласта, так что для полубесконечных пластов вдали от их верхней кромки намагниченность стремится к величине, определяемой традиционной концепцией по формуле (11.9) при $\mathrm{N}=1$. Вблизи же верхней кромки 
размагничивание практически отсутствует. Графики величины $\mathrm{K}_{\mathrm{n}}=\mu_{0}(1+\kappa) \mathrm{I} / \kappa \mathrm{T}_{0} \quad$ для полубесконечных пластов при различных магнитных восприимчивостях показаны на рис. $17 б$. С их помощью для любого пласта, намагниченного в поперечном поле, намагниченность в центральной плоскости определяется по формуле:

$$
\mathrm{I}=\mathrm{K}_{\mathrm{n}} \frac{\kappa \mathrm{T}_{0}}{\mu_{0}(1+\kappa)} .
$$

При z $/ \mathrm{l} \rightarrow 0$ намагниченность таких пластов стремится к $\kappa \mathrm{H}^{\text {прв }}=\kappa \mathrm{T}_{0} / \mu_{0}$.

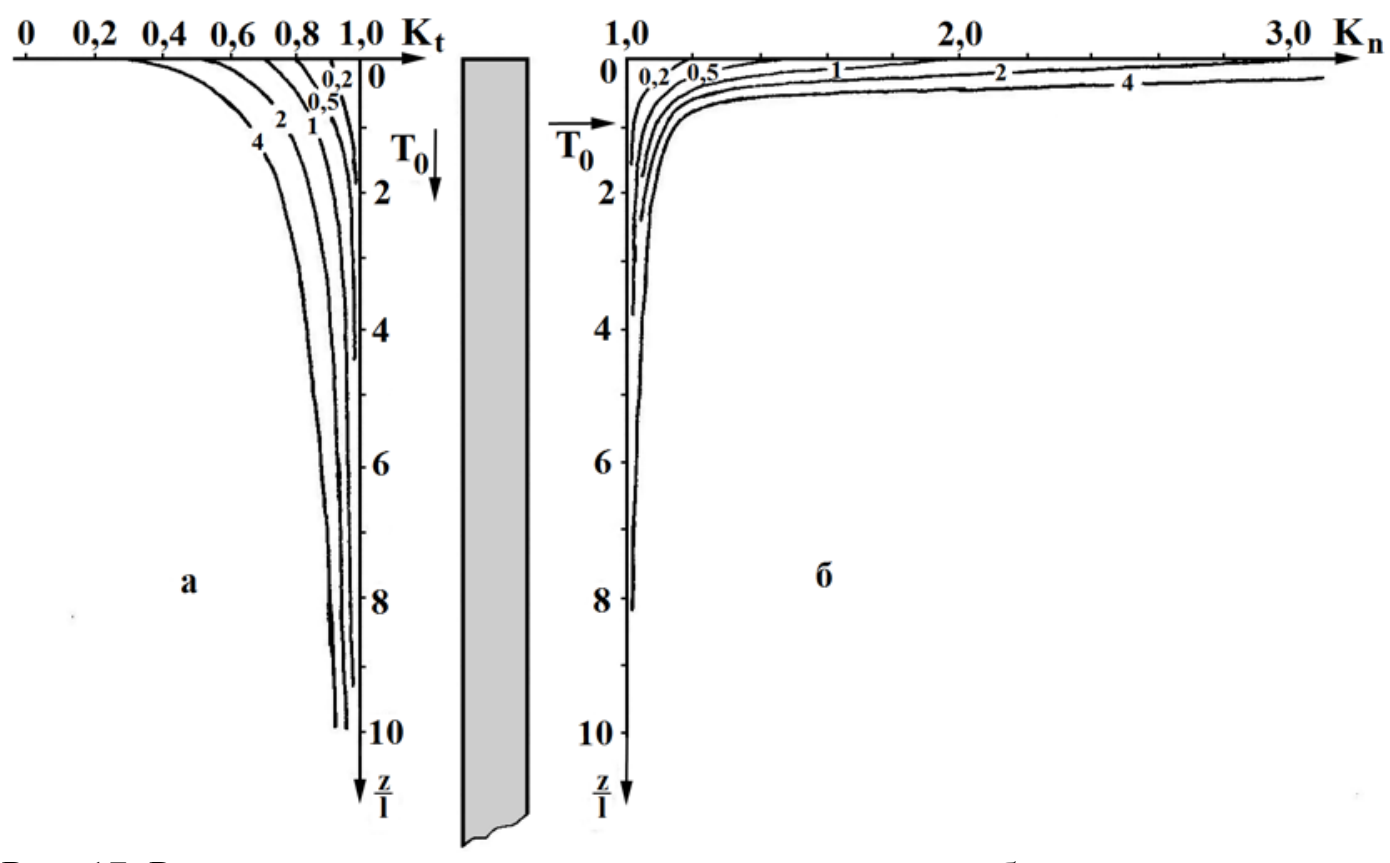

Рис. 17. Распределение намагниченности в тонких полубесконечных пластах, намагниченных в: а) продольном однородном поле; б) поперечном однородном поле

Результаты расчетов показывают, что традиционные способы вычисления намагниченности полубесконечных пластов по формулам для бесконечных слоев применимы лишь для глубокозалегающих частей пластов вдали от их верхней кромки. Степень неоднородности намагниченности пластов возрастает с ростом их магнитной восприимчивости во всем диапазоне встречающихся среди геологических объектов значений. Необходимо отметить, что при дальнейшем росте магнитной восприимчивости, превышающей величину, характерную для магнетита, неоднородность намагничения снова уменьшается, так что при $\kappa \rightarrow \infty$ размагничивающее (вторичное) поле внутри пласта становится однородным и равным первичному, а суммарное поле стремится к нулю. Если пласт ограничен по глубине, то распределение намагниченности у его нижней кромки симметрично. Экстремальное значение в центре продольно намагниченного пласта можно оценить с помощью рис. 16. Для поперечно намагниченного пласта, как следует из рис. 17 , графики $\mathrm{K}_{\mathrm{n}}$ стремятся к асимптотическому значению гораздо быстрее, нежели $\mathrm{K}_{\mathrm{t}}$ для продольно намагниченных пластов, так что уже при $\mathrm{z} / \mathrm{l}=4$ экстремальное значение можно вычислять по формуле (11.9) при $\mathrm{N}=1$.

Перейдем теперь к косонамагниченным пластам. Распределение их намагниченности может быть получено с помощью палеток, изображенных на рис. 17. Для этого первичное поле и остаточную намагниченность надо разложить на продольную и поперечную к пласту компоненты и вычислить составляющие намагниченности по соответствующим формулам. В случае анизотропных пластов для продольной намагниченности надо использовать значение продольной магнитной восприимчивости $\kappa_{\mathrm{t}}$, а для поперечной намагниченности $-\kappa_{n}$. 
Результаты подобных вычислений для изотропного и анизотропного пластов, намагниченных в поле, направленном под углом $45^{\circ}$ к пласту, показаны на рис. 18. На нем видно, что

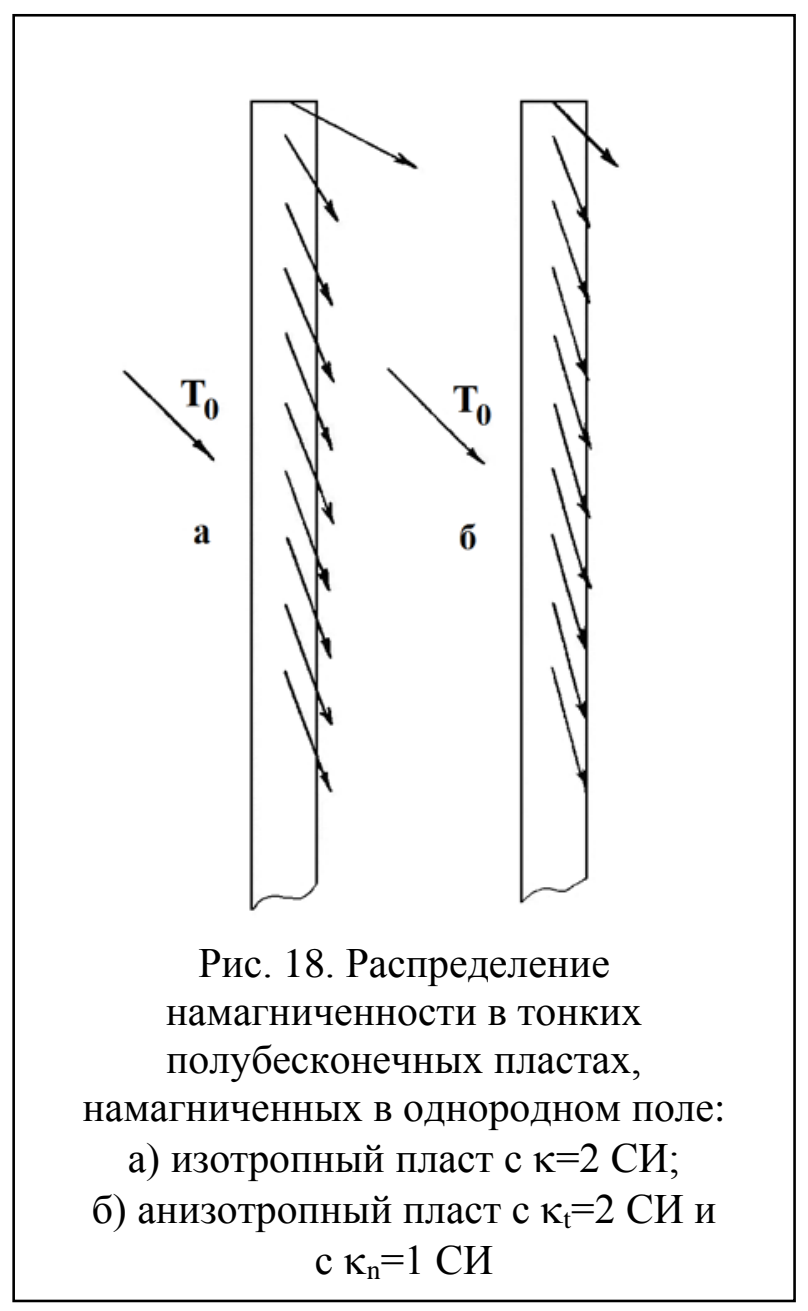
размагничивание приводит к изменению вдоль пласта не только модуля вектора намагниченности, но и его направления, причем лишь на значительном удалении от верхней кромки соблюдаются соотношения, характерные для бесконечного слоя.

Рассмотрим поведение угла $\psi$ между вектором намагниченности и пластом более детально. Обозначим его асимптотическое значение при удалении на большое расстояние от верхней кромки через $\psi_{\infty}$. В общем случае для анизотропного пласта можно получить формулу, связывающую $\psi_{\infty}$ с $\varphi$ и обобщающую (11.3). Для этого разложим напряженность первичного поля $\overline{\mathrm{H}}^{\text {прв}}$, направленного под углом $\varphi$ к пласту, на продольную $\mathrm{H}_{\mathrm{tan}}^{\text {прв }}$ и поперечную $\mathrm{H}_{\text {nor }}^{\text {прв }}$ составляющие:

$$
\begin{aligned}
& \mathrm{H}_{\text {tan }}^{\text {прв }}=\mathrm{H}^{\text {прв }} \cos \varphi, \\
& \mathrm{H}_{\text {nor }}^{\text {прв }}=\mathrm{H}^{\text {прв }} \sin \varphi .
\end{aligned}
$$

Тогда для анизотропного пласта из (11.1) и (11.2) вытекает:

$$
\begin{aligned}
I_{\text {tan }} & =\kappa_{\mathrm{t}} \mathrm{H}^{\text {прв }} \cos \varphi, \\
I_{\text {nor }} & =\frac{\kappa_{\mathrm{n}} H^{\text {прв }} \sin \varphi}{1+\kappa_{\mathrm{n}}} .
\end{aligned}
$$

Тангенс угла $\psi_{\infty}$ выражается через $\mathrm{I}_{\tan }$ и $\mathrm{I}_{\text {nоr }}$ следующим образом:

$$
\operatorname{tg} \psi_{\infty}=\frac{I_{\text {nor }}}{I_{\text {tan }}},
$$

тогда из (11.13) следует

$$
\operatorname{tg} \psi_{\infty}=\frac{\kappa_{\mathrm{n}}}{\kappa_{\mathrm{t}}\left(1+\kappa_{\mathrm{n}}\right)} \operatorname{tg} \varphi .
$$

Для изотропного пласта, когда $\kappa_{\mathrm{t}}=\kappa_{\mathrm{n}}=\kappa$, последняя формула принимает вид (11.3).

Как было отмечено выше, в середине верхней кромки вертикального полубесконечного пласта намагниченность подчиняется закономерностям, справедливым для полупространства. Обозначим угол $\psi$ на верхней кромке как $\psi_{0}$. Для него можно также получить простое соотношение, связывающее $\psi_{0}$ с $\varphi$. Разложим, как и ранее, первичное поле на продольную и поперечную компоненты. Тогда для $\mathrm{I}_{\tan }$ из (11.8) следует

$$
I_{\text {tan }}=\frac{\kappa_{\mathrm{t}} \mathrm{H}^{\mathrm{npB}} \cos \varphi}{1+0,5 \kappa_{\mathrm{t}}},
$$

Для поперечной компоненты намагниченности в центре верхней кромки размагничивание отсутствует и

тогда в соответствии с (11.14)

$$
\mathrm{I}_{\mathrm{nor}}=\kappa_{\mathrm{n}} \mathrm{H}^{\mathrm{np}} \sin \varphi,
$$




$$
\operatorname{tg} \psi_{\infty}=\frac{\kappa_{\mathrm{n}}\left(2+\kappa_{\mathrm{t}}\right)}{2 \kappa_{\mathrm{t}}} \operatorname{tg} \varphi .
$$

Для изотропных пластов при $\kappa_{\mathrm{t}}=\kappa_{\mathrm{n}}=\kappa$ связь упрощается:

$$
\operatorname{tg} \psi_{\infty}=\frac{2+\kappa}{2} \operatorname{tg} \varphi .
$$

Из этой формулы следует, что $\psi_{0}>\varphi$, то есть для изотропных пластов в центре верхней кромки отклонение вектора намагниченности пластов происходит в сторону от пласта, что полностью противоположно выводам традиционной концепции намагничения. Данный факт иллюстрируется на рис. 18а на примере изотропного пласта с магнитной восприимчивостью 2 СИ.

Отклонение вектора намагниченности анизотропных пластов от намагничивающего поля вблизи верхней кромки может в зависимости от коэффициента анизотропии происходить как в ту, так и в другую сторону. Если коэффициент анизотропии мал, то есть пласт близок к изотропному, для него $\psi_{0}>\varphi$; если коэффициент анизотропии велик, то $\psi_{0}>\varphi$. При

$$
\kappa_{\mathrm{n}}=\frac{2 \kappa_{\mathrm{t}}}{2+\kappa_{\mathrm{t}}},
$$

как следует из (11.18), $\psi_{0}=\varphi$, то есть направление вектора намагниченности у верхней кромки совпадает с направлением намагничивающего поля. Данный случай при $\kappa_{\mathrm{t}}=2$ СИ и $\kappa_{\mathrm{n}}=1$ СИ иллюстрируется на рис. 186.

Зная $\psi_{0}$ и $\psi_{\infty}$, можно вычислить и их разность, показывающую, насколько максимально меняется направление вектора намагниченности внутри пласта в его центральной плоскости. Для анизотропных пластов из (11.15) и (11.18) получим

$$
\operatorname{tg}\left(\psi_{0}-\psi_{\infty}\right)=\frac{\kappa_{\mathrm{t}} \kappa_{\mathrm{n}} \operatorname{tg} \varphi\left[\left(2+\kappa_{\mathrm{t}}\right)\left(1+\kappa_{\mathrm{n}}\right)-2\right]}{2 \kappa_{\mathrm{t}}^{2}\left(1+\kappa_{\mathrm{n}}\right)+\kappa_{\mathrm{n}}^{2}\left(2+\kappa_{\mathrm{t}}\right) \operatorname{tg}^{2} \varphi},
$$

для изотропных пластов

$$
\operatorname{tg}\left(\psi_{0}-\psi_{\infty}\right)=\frac{\kappa(3+\kappa) \operatorname{tg} \varphi}{2(1+\kappa)+(2+\kappa) \operatorname{tg}^{2} \varphi} .
$$

В примерах, приведенных на рис. 18, максимальные углы между векторами намагниченности в пластах составляют для изотропного случая $45^{\circ}$, для анизотропного - $31^{\circ}$.

При интерпретации магнитных аномалий для пластов, верхняя кромка которых расположена на глубине, превышающей их мощность настолько, что пласты можно считать тонкими, достаточно учитывать приведенные выше закономерности их намагничения. Если же точки наблюдения располагаются вблизи верхней кромки пласта, то дополнительно надо принимать во внимание неоднородность намагничения, характерную для многоугольных цилиндров. $\mathrm{Ee}$ проявления проиллюстрированы на рис. 19.

Неоднородность намагничения тонких пластов

Рис. 19. Распределение намагниченности изотропного полубесконечного пласта с $\kappa=2$ СИ при намагничении в продольном однородном поле заметно ощущается в создаваемых ими магнитных аномалиях. Рассмотрим для иллюстрации вертикальный пласт с к=2 СИ, намагниченый в однородном вертикальном поле с индукцией 50 мкТл. На рис. 20 показаны графики аномалий $\Delta \mathrm{Z}$ над ним, вычисленные с учетом и без учета размагничивания. Вдали от пласта 
Ю.И. Блох

графики практически совпадают, но в эпицентре аномалии заметно различаются. Поскольку в данном случае части пласта, примыкающие к верхней кромке, намагничены слабее, нежели более глубокие его части, аномалия, вычисленная с учетом размагничивания, имеет меньшую амплитуду. Это изменение формы графиков аномалии оказывает существенное влияние на определение параметров пласта при интерпретации стандартными методами, что будет подробно рассмотрено ниже.

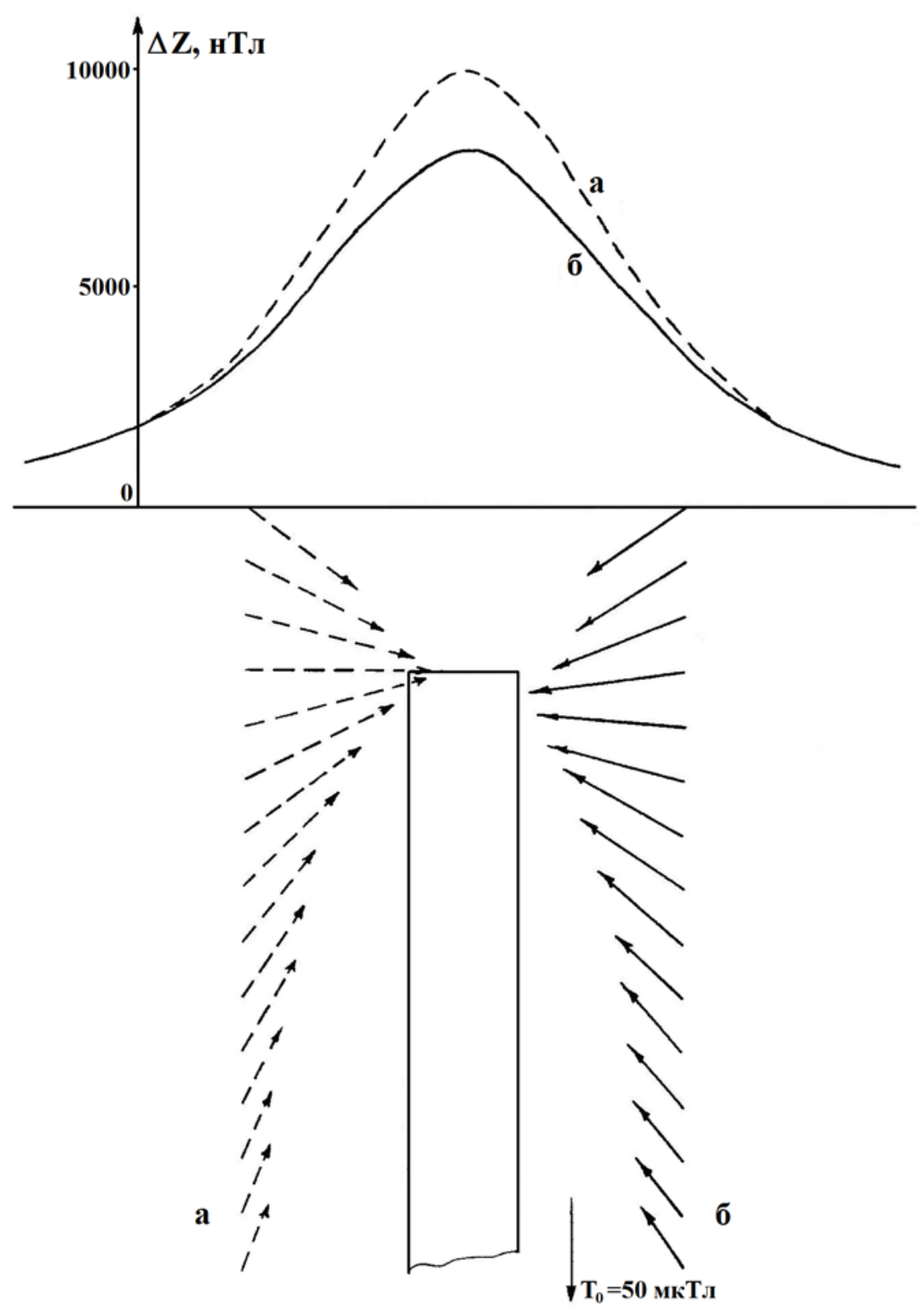

Рис. 20. Влияние размагничивания на магнитную аномалию изотропного полубесконечного пласта с магнитной восприимчивостью 2 СИ: а) аномалия $\Delta \mathrm{Z}$ на дневной поверхности и векторы аномального поля в скважине без учета размагничивания; б) то же с учетом размагничивания

Помимо графиков на рис. 20 изображены векторы аномального магнитного поля в точках по вертикальной скважине, причем для наглядности векторы, полученные с учетом и без учета размагничивания, разнесены слева и справа от пласта. Отметим, что обычные приемы качественной интерпретации аномалий, измеренных в скважинах, для пластов построены на 
обнаружении полюсов, характеризующих верхнюю кромку, по пересечению продолжения векторов аномального поля. Неоднородность намагничения под действием размагничивания приводит к отклонению векторов аномального поля в сторону более намагниченных глубоких частей пласта, в результате чего продолжения векторов аномального поля, измеренных вблизи, но на некотором удалении от пласта, не пересекаются в одной точке. Пренебрежение эффектом размагничивания при этом может привести к значительным погрешностям в определении местоположения пласта. Если же положение пласта известно на основании априорной информации, то подобное поведение векторов аномального поля зачастую интерпретируют как признак наличия в его верхней части мощной зоны окисления, уменьшающей намагниченность. Так как область уменьшения намагниченности под влиянием размагничивания в этом случае в несколько раз превышает мощность пласта, то ошибочное истолкование ее как зоны окисления может в ряде случаев приводить к неверным геологическим выводам.

Подчеркнем, что усложнение поведения векторов поля вблизи верхней кромки пласта вовсе не связано с пропажей особой точки на его верхней кромке. Как будет показано далее, особенность там остается, и методы особых точек для данной задачи остаются весьма эффективными. Нельзя лишь, строго говоря, пользоваться столь грубыми подходами к локализации особенностей на кромках пластов, как примитивный поиск точки пересечения измеренных векторов.

\section{§ 12. Особенности намагничения пластов в поле петли}

Незаземленные петли, являющиеся основными источниками в МИП и в НЧИМ, создают неоднородные первичные поля. В зависимости от расположения точек наблюдения по отношению к петле, первичное поле в них резко меняется как по величине, так и по направлению, поэтому даже для таких объектов, как сильно вытянутые в горизонтальном направлении пласты, прямая задача является трехмерной. Аномальные поля пластов зависят, разумеется, от форм намагничивающих петель и от их расположения по отношению к пластам. Для интерпретации результатов наблюдений принципиальное значение имеют две основные ситуации, когда пласт является крутопадающим или же субгоризонтальным. Рассмотрим вначале случай крутопадающего пласта.

В процессе численного моделирования модель пласта аппроксимировалась 250-ю кубическими элементами, расположенными в вертикальной плоскости в один слой так, что по горизонтали размещалось 25 элементов с длиной ребра $\mathrm{l}=2 \mathrm{~b}$. Намагничивающая квадратная незаземленная петля с длиной стороны $16 \mathrm{~b}$ была расположена симметрично над пластом так, что ее центр находился над центром модели, а две стороны оказывались параллельными пласту. Верхняя кромка пласта находилась на глубине $b$ под плоскостью петли, магнитная восприимчивость была принята равной 1 СИ. Такое расположение модели относительно петли определяет отсутствие компонент намагниченности элементов, направленных перпендикулярно к пласту.

Результаты вычислений представлены на рис. 21a и 21б, где помещены продольные вертикальные разрезы в плоскости пласта. Эффективные намагниченности элементов показаны векторами, модули которых пропорциональны намагниченности. На рис. 21а намагниченность элементов показана без учета размагничивания. Данный рисунок фактически иллюстрирует распределение первичного поля петли, а рис. 216 характеризует влияние размагничивания на распределение намагниченности в пласте. Как видно, наиболее существенно размагничивание влияет на намагниченность верхней части пласта внутри петли, уменьшая ее и тем самым делая распределение намагниченности вблизи верхней кромки более однородным. Этот эффект, естественно, проявляется и в аномалиях.

В методе незаземленной петли результаты полевых наблюдений вертикальной составляющей вектора индукции магнитного поля $\mathrm{B}_{\mathrm{z}}$ обычно представляют в долях нормального поля петли в данной точке $\mathrm{B}_{\mathrm{z}}^{0}$, то есть вычисляют [45]: 
Ю.И. Блох

$$
\mathrm{B}_{\mathrm{z}}^{\mathrm{a}}=\frac{\mathrm{B}_{\mathrm{z}}-\mathrm{B}_{\mathrm{z}}^{0}}{\mathrm{~B}_{\mathrm{z}}^{0}}
$$

Чаще всего аномалию $\mathrm{B}_{\mathrm{z}}^{\mathrm{a}}$ выражают в процентах. Вычисленные аналогично аномалии от модели пласта оказываются практически тождественными по форме аномалиям $\Delta \mathrm{Z}$ от однородно намагниченного пласта, местоположение которого совпадает с истинным положением пласта, но намагниченность которого меньше той, которая была бы у пласта без учета размагничивания. Это приводит к уменьшению амплитуды аномалии $\mathrm{B}_{\mathrm{z}}^{\mathrm{a}}$, в частности, для рассмотренного примера оно составило 19\%.

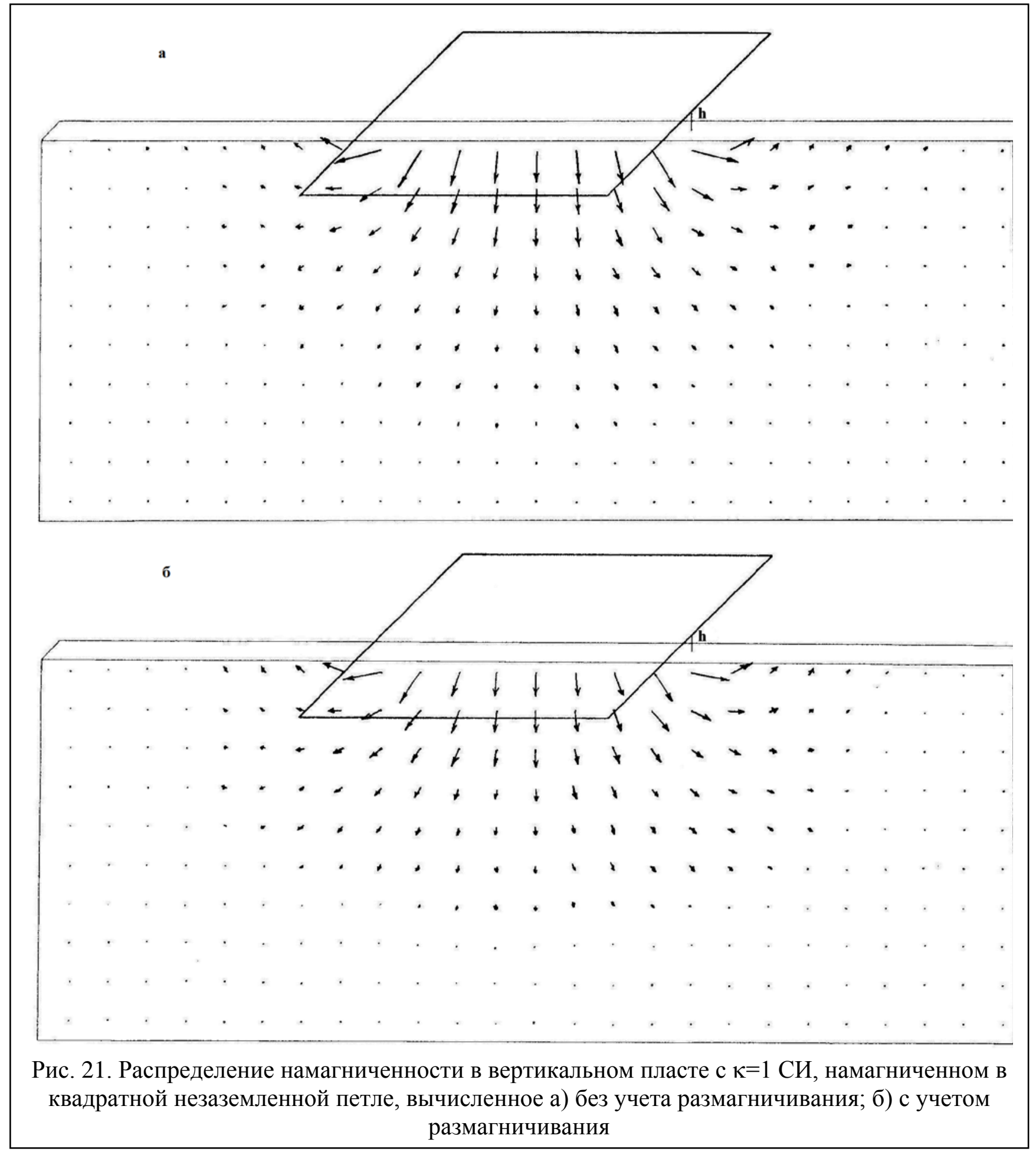


Ю.И. Блох

Теоретические основы комплексной магниторазведки

Таким образом, математическое моделирование демонстрирует некоторую парадоксальность ситуации: оказывается, вертикальный пласт в неоднородном поле незаземленной петли намагничивается у верхней кромки более однородно, нежели в однородном вертикальном поле. Это является результатом наложения двух эффектов: уменьшения первичного поля петли с глубиной и уменьшения намагниченности у верхней кромки под влиянием размагничивания. В итоге оказывается, что мы можем проводить интерпретацию аномалий, получаемых в методе незаземленной петли над крутопадающими пластами по формулам, выведенным в магнитостатике на базе традиционной концепции намагничения, но при этом должны вводить поправки за размагничивание и неоднородность первичного поля.

Перейдем теперь к рассмотрению особенностей намагничения субгоризонтальных пластов в поле петли. Для этого воспользуемся моделью реального геологического объекта в одном из районов КМА. Здесь на глубине 240 м находится субгоризонтальный пласт мощностью около 200 м, форма которого в плане близка к треугольнику со сторонами $6,8,7,2$, и 3,2 км. При его изучении методом незаземленной петли в качестве источника первичного поля применялась прямоугольная петля $3 \times 4$ км, расположенная над пластом как показано на рис. 22 . При проведении математического моделирования объект был аппроксимирован 234-мя кубическими элементами с длиной ребра 200 м. На рис. 23 и 24 представлены вертикальные разрезы модели по линиям АБ и ВГ, на которых векторами изображены проекции намагниченности элементов на плоскости разрезов, вычисленные без учета у с учетом размагничивания при магнитной восприимчивости модели 1,26 СИ.
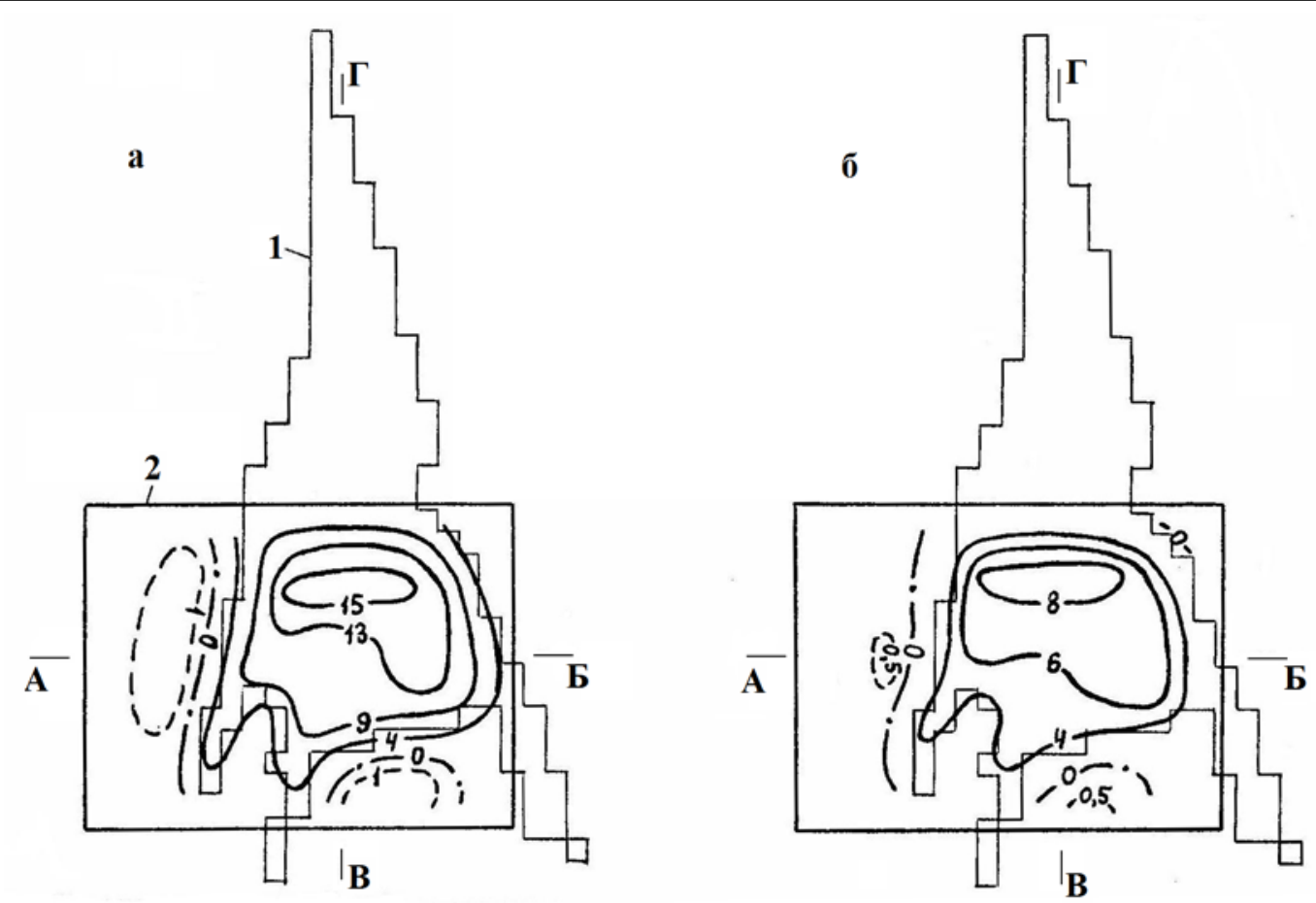

Рис. 22. Карты изолиний $\mathrm{B}_{z}^{\mathrm{a}}(\%)$ горизонтального пласта сложной формы с к=1,26 СИ в поле петли: а) без учета размагничивания; б) с учетом размагничивания;

1 - контур пласта, 2 - контур петли

Анализ рисунков показывает, что для субгоризонтальных пластов влияние размагничивания гораздо более существенно, чем для субвертикальных, причем в разных частях пласта оно проявляется по-разному. В средней трети петли, где намагничивающее поле на каждой горизонтальной плоскости почти однородно, намагниченность подчиняется тем же закономерностям, которые справедливы для пластов, намагниченных в однородном поперечном поле. Их эффективная намагниченность под действием размагничивания 
значительно уменьшается, причем на удалении от боковой кромки уменьшение более значительно. Иная картина наблюдается вблизи сторон петли, где первичное поле неоднородно и резко меняет свое направление. В этой части пласта размагничивание отклоняет вектор намагниченности в сторону пласта, незначительно его уменьшая. Наконец, вдали от петли, где поле снова становится близким к вертикальному, размагничивание так же, как в центре петли, уменьшает намагниченность. Таким образом, для субгоризонтальных пластов в поле петли размагничивание усиливает неоднородность намагничения, если пласт выходит за пределы средней трети петли.

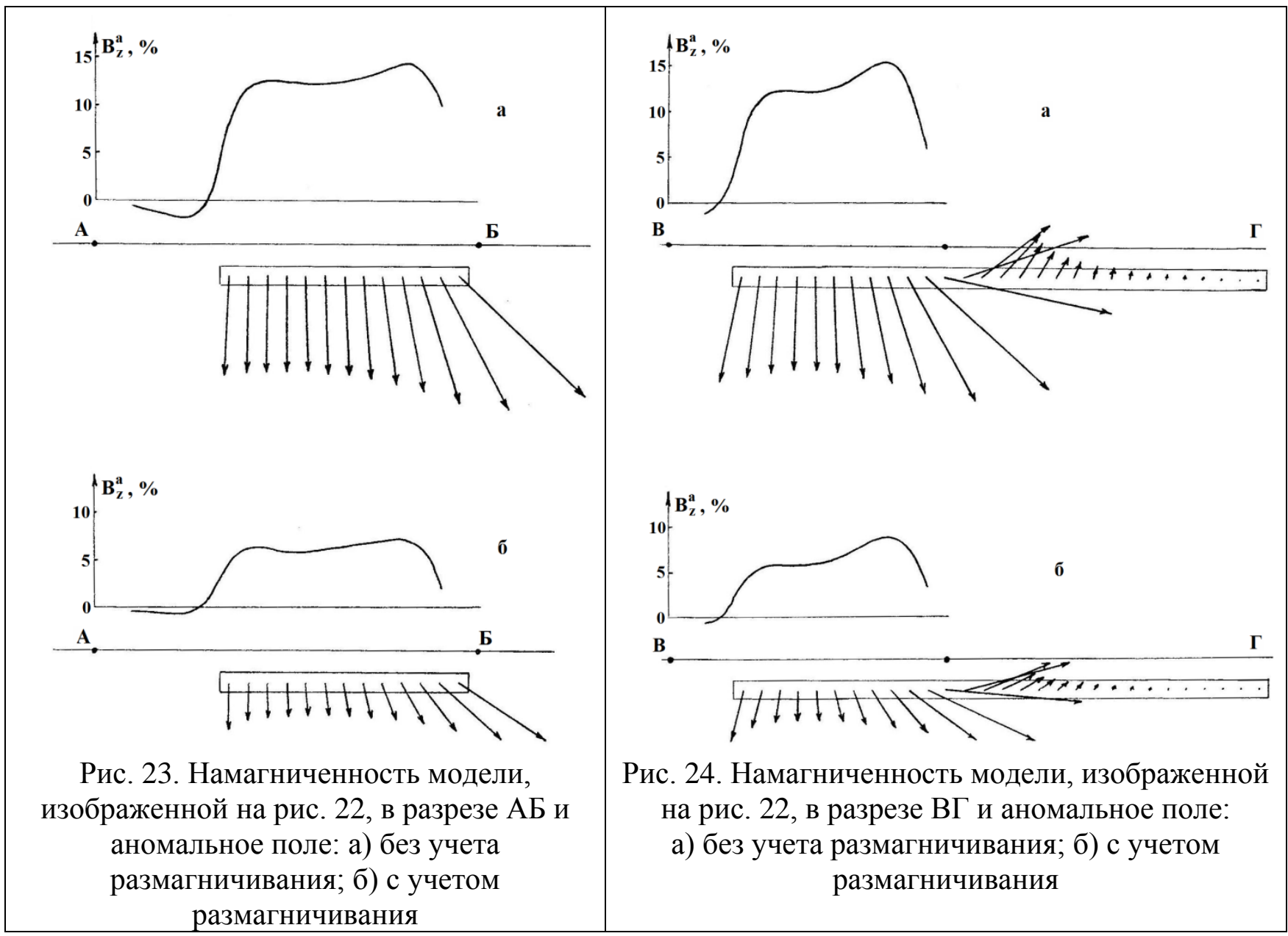

Наиболее характерно для аномалий резкое уменьшение к сторонам петли, вызванное тем, что под проводами намагничение пластов субгоризонтально. Этой же причиной вызвано изменение амплитуды различных экстремумов вблизи сторон петли, так как в этих частях под влиянием размагничивания вектор намагниченности отклоняется ближе к горизонтали. В целом же амплитуда аномалии $\mathrm{B}_{z}^{\mathrm{a}}$ под действием размагничивания в данном случае уменьшается с $15,5 \%$ до $8,84 \%$, то есть в 1,75 раза, что, несомненно, следует учитывать при интерпретации.

\section{§ 13. Намагничение сближенных сильномагнитных объектов}

Рассмотренные модели представляли собой уединенные объекты, что в природе встречается редко, поэтому понимание характера намагничения геологических образований невозможно без понимания взаимовлияния сближенных тел.

Очевидно, что тела намагничиваются не только в земном первичном поле и в собственном аномальном поле, но также и в аномальном поле соседних тел, а это приводит к эффекту 
взаимовлияния, в частности, к тому, что аномалия над объектом, состоящим из двух тел, не равна сумме их аномалий [39]. При этом надо иметь в виду, что данный эффект проявляется двояко: с одной стороны, приводя к изменению магнитного момента составного объекта и, с другой стороны, изменяя морфологию аномалии. Попробуем разобраться в этих аспектах.

Простой, но достаточно точный способ оценки изменения магнитного момента составного объекта в результате взаимного влияния тел был предложен автором в начале $70-\mathrm{x}$ годов прошлого века [20-22]. В его основе лежит упрощение, заключающееся в том, что изучаемые тела считаются однородно намагничивающими друг друга, причем напряженность аномального поля во всем объеме соседнего тела полагается равной величине в его центре. Это упрощение дает возможность оценить взаимовлияние путем решения системы линейных алгебраических уравнений, к которой сводится интегральное уравнение для намагниченности, распределенной по объему изучаемого тела. Применение данного подхода наиболее эффективно для оценки взаимовлияния эллипсоидов и их частных форм, поскольку они, будучи уединенными, в однородных полях намагничиваются однородно. Рассмотрим в связи с этим сущность методики применительно к двум взаимодействующим анизотропным эллипсоидам.

Пусть два однородных анизотропных эллипсоида с тензорами магнитной восприимчивости $\kappa_{1}$ и к $\kappa_{2}$, центры которых расположены в точках $a_{1}$ и $a_{2}$, имеют начальные однородные намагниченности без учета их взаимовлияния $\overline{\mathrm{I}}_{01}$ и $\overline{\mathrm{I}}_{02}$. Тензоры размагничивания эллипсоидов, учитывающие их ориентировку в пространстве, $\mathrm{N}_{1}$ и $\mathrm{N}_{2}$. Обозначим матрицу, которая описывает аномальное поле, создаваемое первым эллипсоидом в центре второго $\mathrm{Q}\left(\mathrm{a}_{1}, \mathrm{a}_{2}\right)$, а матрицу, которая описывает аномальное поле, создаваемое вторым эллипсоидом в центре первого $\mathrm{Q}\left(\mathrm{a}_{2}, \mathrm{a}_{1}\right)$. В этих обозначениях система уравнений, описывающих взаимовлияние эллипсоидов, запишется в следующем виде, обобщающим систему (7.1) для учета попарного взаимовлияния шаров и цилиндров:

$$
\begin{aligned}
& \overline{\mathrm{I}}_{1}=\left[\mathrm{E}+\kappa_{1} \mathrm{~N}_{1} \mathrm{Q}\left(\mathrm{a}_{2}, \mathrm{a}_{1}\right)\right]^{-1}\left[\overline{\mathrm{I}}_{01}+\kappa_{1} \mathrm{Q}\left(\mathrm{a}_{2}, \mathrm{a}_{1}\right) \overline{\mathrm{I}}_{2}\right], \\
& \overline{\mathrm{I}}_{2}=\left[\mathrm{E}+\kappa_{2} \mathrm{~N}_{2} \mathrm{Q}\left(\mathrm{a}_{1}, \mathrm{a}_{2}\right)\right]^{-1}\left[\overline{\mathrm{I}}_{02}+\kappa_{2} \mathrm{Q}\left(\mathrm{a}_{1}, \mathrm{a}_{2}\right) \overline{\mathrm{I}}_{1}\right] .
\end{aligned}
$$

Решая ее, аналогично (7.1), получим

$$
\begin{aligned}
& \overline{\mathrm{I}}_{1}=\mathrm{A}\left(\mathrm{a}_{2}, \mathrm{a}_{1}\right) \overline{\mathrm{I}}_{01}+\mathrm{B}\left(\mathrm{a}_{2}, \mathrm{a}_{1}\right) \overline{\mathrm{I}}_{02}, \\
& \overline{\mathrm{I}}_{2}=\mathrm{A}\left(\mathrm{a}_{1}, \mathrm{a}_{2}\right) \overline{\mathrm{I}}_{02}+\mathrm{B}\left(\mathrm{a}_{1}, \mathrm{a}_{2}\right) \overline{\mathrm{I}}_{01},
\end{aligned}
$$

где

$$
\begin{aligned}
A\left(a_{2}, a_{1}\right)= & \left\{E+\kappa_{1} N_{1} Q\left(a_{2}, a_{1}\right)+\kappa_{1} Q\left(a_{2}, a_{1}\right)\left[E+\kappa_{2} N_{2} Q\left(a_{1}, a_{2}\right)\right]^{-1} \kappa_{2} Q\left(a_{1}, a_{2}\right)\right\}^{-1}, \\
& B\left(a_{2}, a_{1}\right)=A\left(a_{2}, a_{1}\right) \kappa_{1} Q\left(a_{2}, a_{1}\right)\left[E+\kappa_{2} N_{2} Q\left(a_{1}, a_{2}\right)\right]^{-1}, \\
A\left(a_{1}, a_{2}\right)= & \left\{E+\kappa_{2} N_{2} Q\left(a_{1}, a_{2}\right)+\kappa_{2} Q\left(a_{1}, a_{2}\right)\left[E+\kappa_{1} N_{1} Q\left(a_{2}, a_{1}\right)\right]^{-1} \kappa_{1} Q\left(a_{2}, a_{1}\right)\right\}^{-1}, \\
& B\left(a_{1}, a_{2}\right)=A\left(a_{1}, a_{2}\right) \kappa_{2} Q\left(a_{1}, a_{2}\right)\left[E+\kappa_{1} N_{1} Q\left(a_{2}, a_{1}\right)\right]^{-1} .
\end{aligned}
$$

В случае одинаковых однородных изотропных эллипсоидов с равными первоначальными намагниченностями матрицы $\mathrm{Q}$ равны, а именно $\mathrm{Q}\left(\mathrm{a}_{1}, \mathrm{a}_{2}\right)=\mathrm{Q}\left(\mathrm{a}_{2}, \mathrm{a}_{1}\right)$, если оси эллипсоидов параллельны друг другу. В наиболее простых ситуациях, когда первоначальная намагниченность ориентирована по осям эллипсоидов, а сами они расположены симметрично, взаимовлияние может быть описано с помощью коэффициента взаимовлияния $\mathrm{F}$ следующим образом:

$$
\overline{\mathrm{I}}_{1}=\overline{\mathrm{I}}_{2}=\mathrm{F} \overline{\mathrm{I}}_{01}=\mathrm{F} \overline{\mathrm{I}}_{02},
$$

где

$$
\mathrm{F}=\frac{1}{1-\mathrm{Q}}
$$

Пусть $\overline{\mathrm{I}}_{01}=\overline{\mathrm{I}}_{02}$ перпендикулярны плоскости симметрии двух одинаковых эллипсоидов, проходящей через их центры. Тогда, обозначив через $\mathrm{R}$ расстояние между центрами эллипсоидов, можно записать следующие выражения, характеризующие их функции Q. Для шара радиуса $\mathrm{R}_{ш}$ : 


$$
\mathrm{Q}=-\frac{\mu-1}{\mu+2} \cdot \frac{\mathrm{R}_{\mathrm{m}}^{3}}{\mathrm{R}^{3}} .
$$

Для горизонтального кругового цилиндра радиуса $\mathrm{R}_{\mathrm{L}}$ :

$$
\mathrm{Q}=-\frac{\mu-1}{\mu+1} \cdot \frac{\mathrm{R}_{\mathrm{u}}^{2}}{\mathrm{R}^{2}}
$$

Для эллиптического цилиндра с полуосями $\mathrm{R}_{\mathrm{a}}$ и $\mathrm{R}_{\mathrm{b}}$, $\mathrm{y}$ которого $\mathrm{R}_{\mathrm{a}}$ параллельна $\overline{\mathrm{I}}_{0}$ :

$$
\mathrm{Q}=-\frac{\mathrm{R}_{\mathrm{a}} \mathrm{R}_{\mathrm{b}}\left(\mathrm{R}_{\mathrm{a}}+\mathrm{R}_{\mathrm{b}}\right)(\mu-1)}{\left(\mathrm{R}_{\mathrm{a}}+\mu \mathrm{R}_{\mathrm{b}}\right)\left(\mathrm{R}^{2}+\mathrm{R}_{\mathrm{a}}^{2}-\mathrm{R}_{\mathrm{b}}^{2}+\mathrm{R} \sqrt{\mathrm{R}^{2}+\mathrm{R}_{\mathrm{a}}^{2}-\mathrm{R}_{\mathrm{b}}^{2}}\right)} .
$$

Эта формула справедлива как для вытянутого вдоль поля эллиптического цилиндра $\left(\mathrm{R}_{\mathrm{a}}>\mathrm{R}_{\mathrm{b}}\right)$, так и для сжатого $\left(\mathrm{R}_{\mathrm{a}}<\mathrm{R}_{\mathrm{b}}\right)$. При $\mathrm{R}_{\mathrm{a}}=\mathrm{R}_{\mathrm{b}}=\mathrm{R}_{\mathrm{L}}$ она обращается в (13.7). Для эллипсоидов вращения вокруг оси $\mathrm{R}_{\mathrm{a}}$ различаются два случая. Для вытянутых вдоль поля эллипсоидов $\left(\mathrm{R}_{\mathrm{a}}>\mathrm{R}_{\mathrm{b}}\right)$ :

$$
\mathrm{Q}=-\frac{\mathrm{R}_{\mathrm{a}} \mathrm{R}_{\mathrm{b}}^{2}(\mu-1)}{\mathrm{f}^{3}+(\mu-1) \mathrm{R}_{\mathrm{b}}^{2}\left(\mathrm{R}_{\mathrm{a}} \ln \frac{\mathrm{R}_{\mathrm{a}}+\mathrm{f}}{\mathrm{R}_{\mathrm{b}}}-\mathrm{f}\right)}\left(\ln \frac{\sqrt{\mathrm{R}^{2}+\mathrm{f}^{2}}+\mathrm{f}}{\mathrm{R}}-\frac{\mathrm{f}}{\sqrt{\mathrm{R}^{2}+\mathrm{f}^{2}}}\right),
$$

где

$$
f=\sqrt{R_{a}^{2}-R_{b}^{2}} .
$$

Для сжатых вдоль поля эллипсоидов вращения $\mathrm{R}_{\mathrm{b}}>\mathrm{R}_{\mathrm{a}}$ и

$$
\left.\mathrm{Q}=-\frac{\mathrm{R}_{\mathrm{a}} \mathrm{R}_{\mathrm{b}}^{2}(\mu-1)}{\mathrm{f}^{2}+(\mu-1) \mathrm{R}_{\mathrm{b}}^{2}\left(1-\frac{\mathrm{R}_{\mathrm{a}}}{\mathrm{f}} \operatorname{arctg} \frac{\mathrm{f}}{\mathrm{R}_{\mathrm{a}}}\right.}\right)\left(\frac{1}{\sqrt{\mathrm{R}^{2}-\mathrm{f}^{2}}}-\frac{1}{\mathrm{f}} \operatorname{arctg} \frac{\mathrm{f}}{\sqrt{\mathrm{R}^{2}-\mathrm{f}^{2}}}\right),
$$

где

$$
\mathrm{f}=\sqrt{\mathrm{R}_{\mathrm{b}}^{2}-\mathrm{R}_{\mathrm{a}}^{2}} .
$$

В случае, когда $\overline{\mathrm{I}}_{01}=\overline{\mathrm{I}}_{02}$ параллельны одной из осей одинаковых эллипсоидов, а центры их лежат на одной линии вдоль этой оси, соответствующие выражения имеют следующий вид. Для шара

$$
\mathrm{Q}=\frac{\mu-1}{\mu+2} \cdot \frac{2 \mathrm{R}_{\mathrm{m}}^{3}}{\mathrm{R}^{3}}
$$

для кругового цилиндра

$$
\mathrm{Q}=\frac{\mu-1}{\mu+1} \cdot \frac{\mathrm{R}_{\mathrm{u}}^{2}}{\mathrm{R}^{2}},
$$

для эллиптического цилиндра, полуось $\mathrm{R}_{\mathrm{a}}$ которого параллельна полю,

$$
\mathrm{Q}=\frac{\mathrm{R}_{\mathrm{a}} \mathrm{R}_{\mathrm{b}}\left(\mathrm{R}_{\mathrm{a}}+\mathrm{R}_{\mathrm{b}}\right)(\mu-1)}{\left(\mathrm{R}_{\mathrm{a}}+\mu \mathrm{R}_{\mathrm{b}}\right)\left(\mathrm{R}^{2}-\mathrm{R}_{\mathrm{a}}^{2}+\mathrm{R}_{\mathrm{b}}^{2}+\mathrm{R} \sqrt{\mathrm{R}^{2}-\mathrm{R}_{\mathrm{a}}^{2}+\mathrm{R}_{\mathrm{b}}^{2}}\right)},
$$

для вытянутого вдоль поля эллипсоида вращения

$$
\mathrm{Q}=\frac{\mathrm{R}_{\mathrm{a}} \mathrm{R}_{\mathrm{b}}^{2}(\mu-1)}{\mathrm{f}^{3}+(\mu-1) \mathrm{R}_{\mathrm{b}}^{2}\left(\mathrm{R}_{\mathrm{a}} \ln \frac{\mathrm{R}_{\mathrm{a}}+\mathrm{f}}{\mathrm{R}_{\mathrm{b}}}-\mathrm{f}\right)}\left(\frac{\mathrm{fR}}{\mathrm{R}^{2}-\mathrm{f}^{2}}-\frac{1}{2} \ln \frac{\mathrm{R}+\mathrm{f}}{\mathrm{R}-\mathrm{f}}\right),
$$

где f определяется соотношением (13.9). Для сжатого эллипсоида соответственно

$$
\mathrm{Q}=\frac{\mathrm{R}_{\mathrm{a}} \mathrm{R}_{\mathrm{b}}^{2}(\mu-1)}{\mathrm{f}^{2}+(\mu-1) \mathrm{R}_{\mathrm{b}}^{2}\left(1-\frac{\mathrm{R}_{\mathrm{a}}}{\mathrm{f}} \operatorname{arctg} \frac{\mathrm{f}}{\mathrm{R}_{\mathrm{a}}}\right)}\left(\frac{1}{\mathrm{f}} \operatorname{arctg} \frac{\mathrm{f}}{\mathrm{R}}-\frac{\mathrm{R}}{\mathrm{R}^{2}+\mathrm{f}^{2}}\right),
$$


Ю.И. Блох

Теоретические основы комплексной магниторазведки

причем f здесь определяется соотношением (13.11). Пользуясь приведенными элементарными выражениями, достаточно легко оценить вероятное взаимовлияние пары практически любых объектов.

Таким образом, коэффициентом взаимовлияния тел является отношение $\mathrm{F}=\mathrm{M}_{\text {ист }} / \mathrm{M}_{\text {сум}}$, где $\mathrm{M}_{\text {ист }}$ - реальный магнитный момент совокупности двух тел, а $\mathrm{M}_{\text {сум }}$ - сумма магнитных моментов каждого из этих тел, вычисленных в пренебрежении влиянием соседа. В зависимости от расположения тел по отношению к направлению первичного намагничивающего поля коэффициент взаимовлияния может быть большим или меньшим единицы. Если тела смещены друг относительно друга по направлению первичного поля, $\mathrm{F}>1$, если они смещены перпендикулярно к направлению первичного поля, $\mathrm{F}<1$. На рис. 25 показаны графики $\mathrm{F}$ для одинаковых соприкасающихся эллиптических цилиндров и эллипсоидов вращения при разных величинах магнитной восприимчивости в системе СИ в зависимости от их относительной вытянутости $\mathrm{k}$, равной отношению полуоси, ориентированной вдоль намагничивающего поля, $\mathrm{k}$ полуоси, ориентированной перпендикулярно полю. С помощью данных графиков легко оценить общее изменение момента в результате взаимовлияния тел.

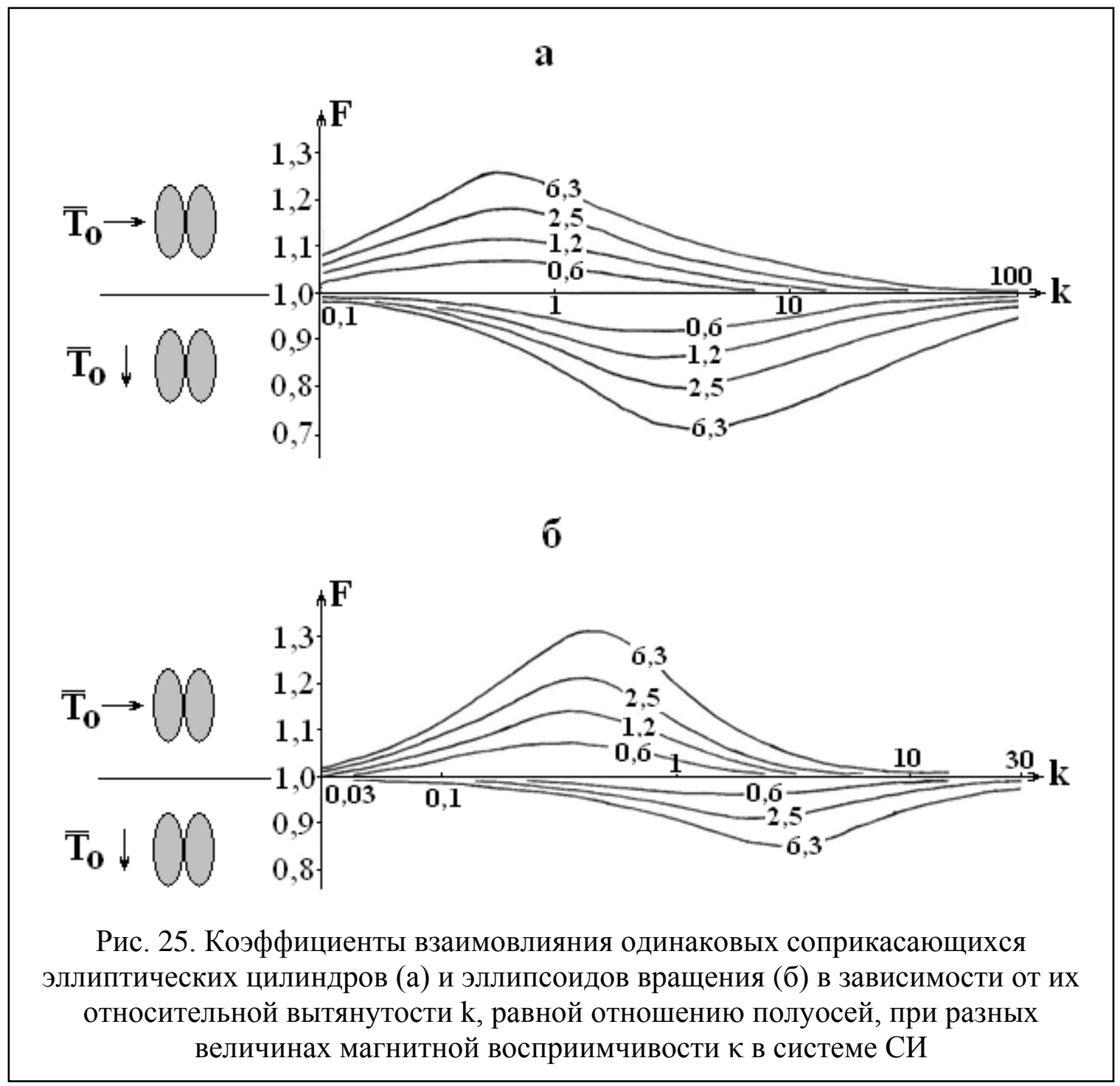

Характер уменьшения взаимовлияния с удалением тел друг от друга можно представить с помощью рис. 26. На нем изображены графики зависимости коэффициента взаимовлияния $\mathrm{F}$ от относительного удаления одинаковых круговых цилиндров и шаров $\mathrm{R} / \mathrm{R}_{\mathrm{L}}$ и $\mathrm{R} / \mathrm{R}_{\mathrm{ш}}$ при различных значениях магнитной восприимчивости. C помощью данных графиков легко оценить, на каком 
Ю.И. Блох

Теоретические основы комплексной магниторазведки

расстоянии друг от друга взаимодействующие шары и цилиндры с данной магнитной восприимчивостью создают дополнительную намагниченность, не превышающую некоторой заданной величины, например, среднеквадратической погрешности выполненной съемки.

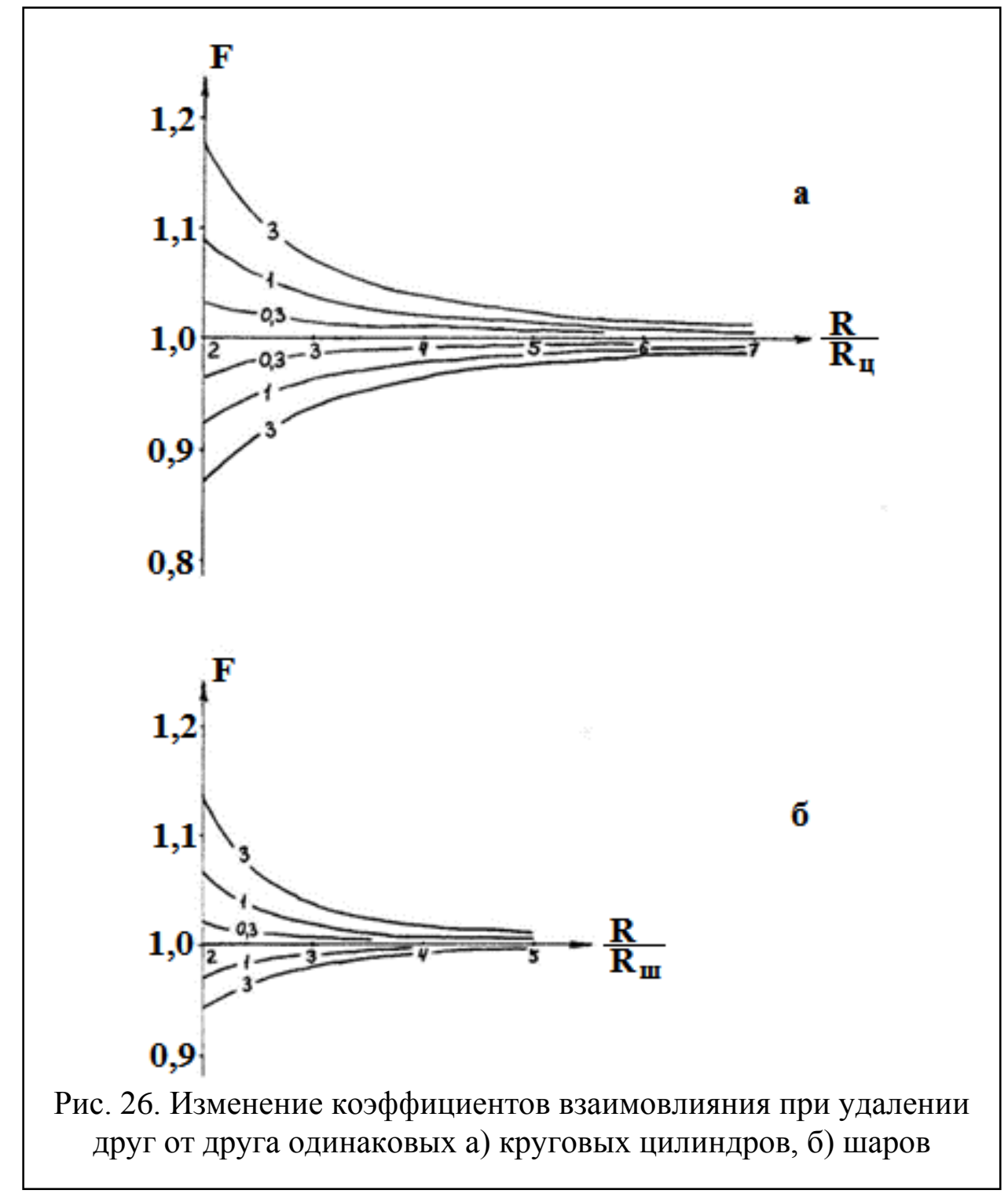

Таким образом, анализ коэффициентов $\mathrm{F}$ дает возможность достаточно просто и эффективно оценить изменение магнитного момента пары тел в результате их взаимного влияния.

Другим проявлением эффекта взаимовлияния является изменение морфологии аномалии над сближенными объектами по сравнению с суммой аномалий отдельных объектов. Разность между общей аномалией и суммой частных аномалий будем называть аномалией взаимовлияния.

Одной из самых важных для комплексной магниторазведки интерпретационных моделей, характерных для месторождений железистых кварцитов, является пачка сближенных крутопадающих пластов. Соответственно, для анализа возможной морфологии аномалий взаимовлияния целесообразно воспользоваться именно этой моделью. На рис. 27 показаны магнитные аномалии пачки пластов с типичной для железистых кварцитов магнитной восприимчивостью 1 СИ, намагниченных в вертикальном магнитном поле с индукцией 50 мкТл. Каждый из пластов простирается на глубину, в 33 раза большую, нежели его мощность. В процессе численного решения интегрального уравнения каждый из пластов 
Ю.И. Блох

разбивался на 300 элементов $(3 \times 100)$. На рисунке четко видно, что взаимовлияние изменило не только магнитный момент, но и морфологию аномалии $\Delta \mathrm{T}$. При этом общий размах аномалии взаимовлияния составил 4280 нТл, т.е. более $30 \%$ размаха самой реальной аномалии. Понятно, что столь большим влиянием пренебрегать никак нельзя.

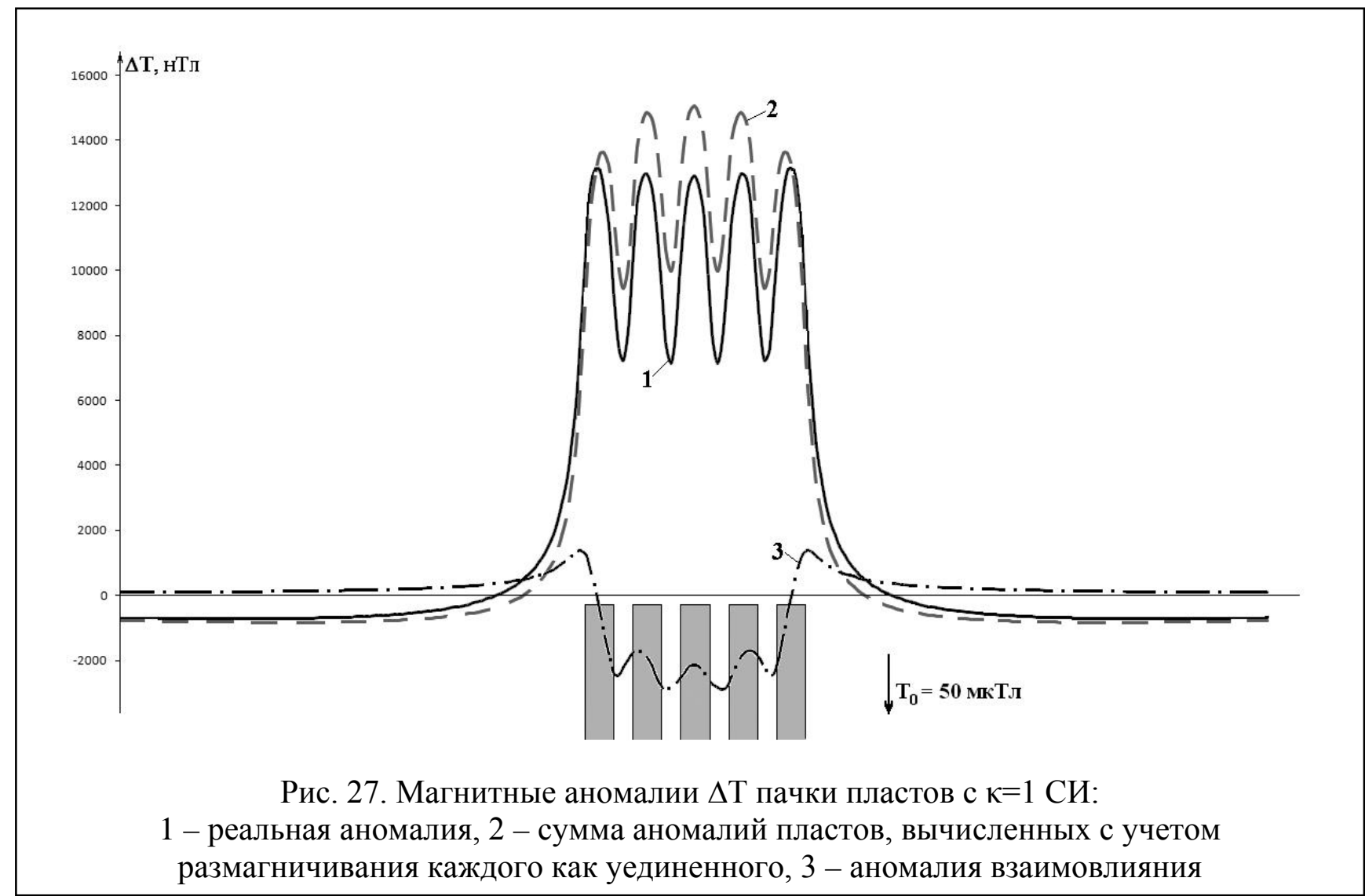

Чтобы понять характер изменения аномалий взаимовлияния в зависимости от направления первичного поля, обратимся к рис. 28. На нем представлены аномалии $\Delta \mathrm{Z}$ взаимовлияния в пачке из двух вертикальных пластов разной мощности $\mathrm{c} \kappa=1$ СИ при направлении первичного поля вертикально (вдоль пластов) и горизонтально. Видно, что аномалии различаются не только морфологически, но и по амплитуде, что физически легко объяснимо. Дело в том, что если пласт вытянут вдоль поля, размагничивание для него в целом мало, и на этом фоне взаимовлияние проявляется гораздо резче, нежели для пласта, вытянутого перпендикулярно полю, где и без учета взаимовлияния размагничивание близко к максимально возможному.

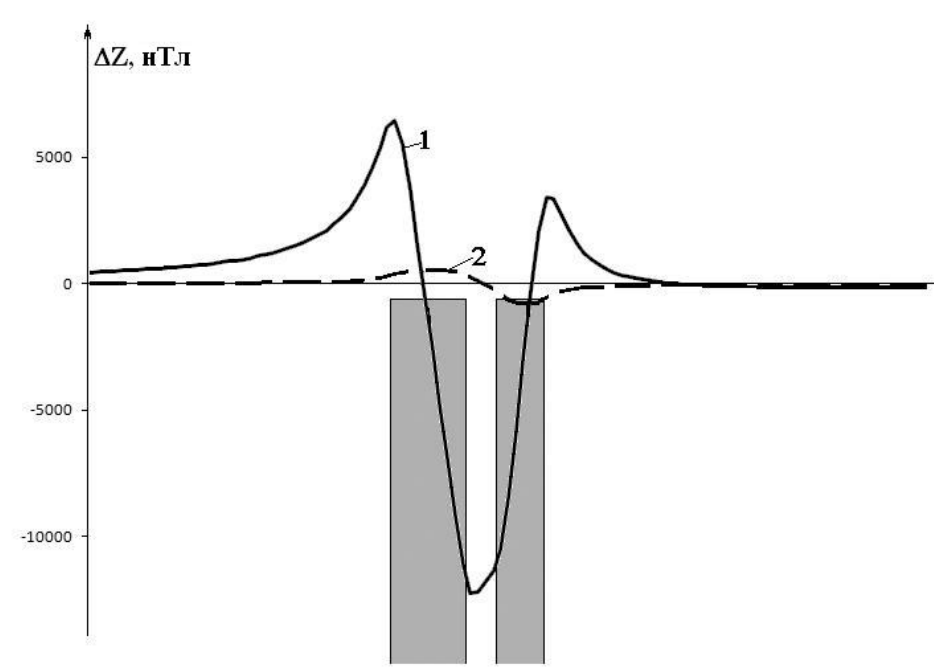

Рис. 28. Аномалии $\Delta \mathrm{Z}$ взаимовлияния в пачке из двух вертикальных пластов разной мощности с магнитной восприимчивостью 1 СИ при направлении первичного поля вертикально (1) и горизонтально (2) Для произвольного направления первичного поля морфологию аномалий взаимовлияния можно 
легко представить себе с помощью принципа суперпозиции полей, комбинируя аномалии, представленные на рисунке.

При разведке месторождений магнетитовых руд их эффективную магнитную восприимчивость часто пересчитывают с помощью регрессионных зависимостей в содержание магнетитового железа. Для железистых кварцитов подобный прием обычно вполне обоснован, тогда как на скарново-магнетитовых месторождениях к нему приходится относиться осторожно. Дело в том, что там относительно богатые руды могут быть сосредоточены в небольших телах, неоднородно распределенных по некоторому макроскопическому блоку. При этом эффективная к блока понижена, и интерпретатор затрудняется в принятии решения: то ли блок содержит лишь бедные и забалансовые руды, более-менее однородно распределенные по нему, и его дальнейшее изучение нецелесообразно, то ли там имеются богатые рудные тела и есть смысл продолжать исследования с целью их локализации в его пределах.

Чтобы уверенно преодолевать подобные сомнения, надо знать, каким образом неоднородно распределенные магнитные объекты влияют на эффективную магнитную восприимчивость и на каких расстояниях до объектов неоднородность можно выявлять по данным комплексной магниторазведки. Иначе говоря, надо знать, как проявляется взаимовлияние множества неоднородностей в пределах геологических объектов. Эти знания можно получить путем математического моделирования.

Рассмотрим двумерную задачу для неоднородного квадратного цилиндра с разными распределениями магнитных включений в нем. Намагничивающее поле при этом будем считать вертикальным, что характерно для МИП и НЧИМ, а его магнитную индукцию равной 50 мкТл, то есть примерно соответствующей земному полю, что позволяет оценивать эффекты в привычных магниторазведчикам единицах. Напряженность намагничивающего поля, таким образом, равна 39,7887358 А/м. Здесь сразу же стоит подчеркнуть, что в отличие от обычной магниторазведки, где за счет остаточного намагничения преимущественное

направление векторов намагниченности может самым существенным образом отличаться от направления намагничивающего поля, в комплексной магниторазведке такого быть не может. Направления векторов намагниченности могут различаться в разных точках объектов, особенно вблизи вершин многогранных тел, но общий магнитный момент может отклоняться от намагничивающего поля только за счет размагничивания объекта, и это может служить источником дополнительной информации о его морфологии и структуре. Магнитную восприимчивость включений будем считать равной 3 СИ, что

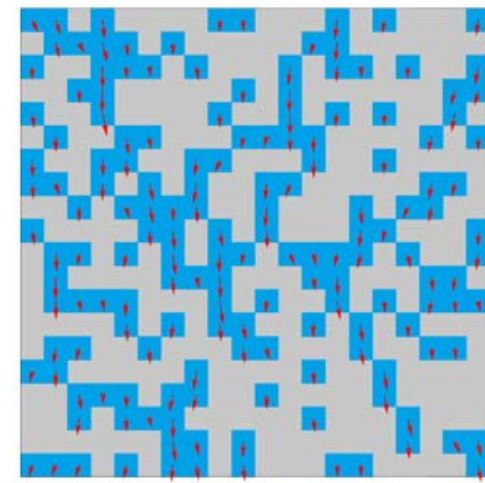
$40 \%$

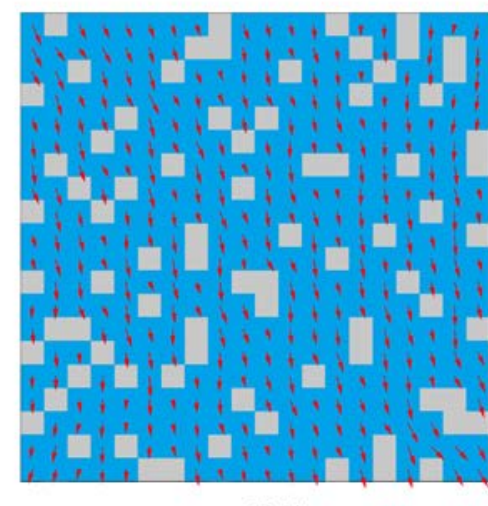

$80 \%$

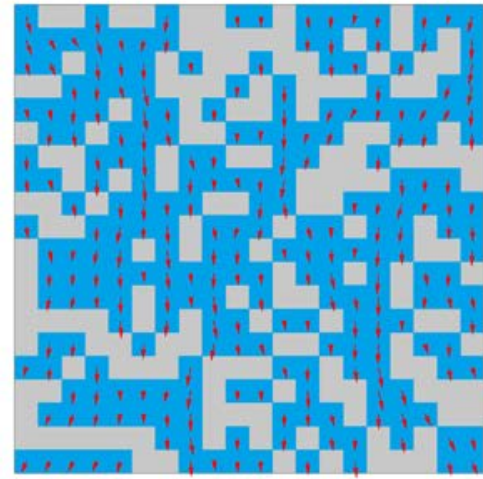

$60 \%$

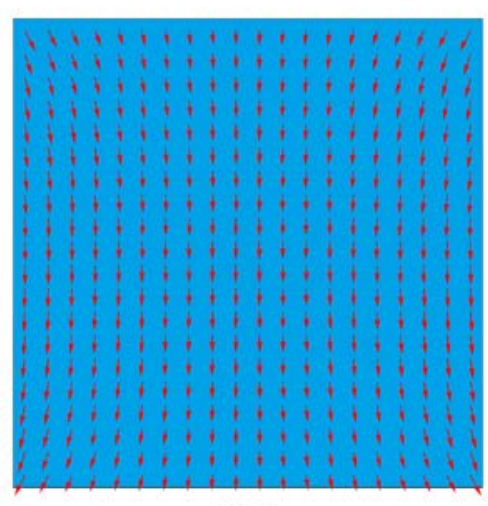

$100 \%$

Рис. 29. Векторы намагниченности магнитных элементов при разных их объемных концентрациях в квадратном цилиндре

соответствует достаточно богатым рудам. Конечно, встречаются рудные тела, сложенные почти 
чистым магнетитом с к около 20 СИ, но они как природные редкости требуют к себе особого отношения.

На рис. 29 показаны распределения намагниченности включений при разном их содержании. При формировании моделей у вершин квадрата непременно помещались магнитные элементы, чтобы в аномальных полях четко проявлялись совпадающие с этими вершинами основные особые точки функций, описывающих эти поля, тогда как остальные включения размещались случайно на основе использования генератора случайных чисел. Качественное сопоставление представленных распределений показывает, что в них четко проявляются две характерных особенности:

1) при любых концентрациях магнитных включений определяющей основой распределения намагниченности является форма макроскопического объекта, содержащего их: в данном случае квадратного цилиндра, благодаря чему векторы намагниченности вблизи его вершин закономерно стремятся к биссектрисам соответствующих углов;

2) чем меньше концентрация магнитных включений, тем больше влияние конкретной формы каждого из локальных включений на их собственную намагниченность.

Особый интерес представляют компоненты вектора средней намагниченности, вычисляемого как отношение линейного магнитного момента модели к площади ее поперечного сечения. Они сведены в таблицу 8, и одна из колонок в ней показывает эффективную $\kappa$, определяемую как отношение вертикальной компоненты средней намагниченности к напряженности намагничивающего поля.

Таблица 8.

Намагничение неоднородных объектов

\begin{tabular}{|c|c|c|c|c|}
\hline \multirow[t]{2}{*}{$\begin{array}{l}\text { Концентрация } \\
\text { включений, \% }\end{array}$} & \multicolumn{2}{|c|}{$\begin{array}{c}\text { Компоненты вектора } \\
\text { средней намагниченности }\end{array}$} & \multirow{2}{*}{$\begin{array}{c}\text { Эффективная } \\
\text { магнитная } \\
\text { восприимчивость, } \\
\text { СИ }\end{array}$} & \multirow{2}{*}{$\begin{array}{c}\text { Кажущаяся } \\
\text { концентрация } \\
\text { включений, \% }\end{array}$} \\
\hline & $\mathrm{I}_{\mathrm{X}}, \mathrm{A} / \mathrm{M}$ & $\mathrm{I}_{\mathrm{z}}, \mathrm{A} / \mathrm{M}$ & & \\
\hline 100 & 0 & 49,181 & 1,236 & 100 \\
\hline 80 & 0,086 & 40,142 & 1,009 & 81,6 \\
\hline 60 & 0,232 & 30,010 & 0,754 & 61,0 \\
\hline 40 & 0,143 & 20,260 & 0,509 & 41,2 \\
\hline
\end{tabular}

Последняя колонка таблицы содержит кажущиеся концентрации включений - они фактически являются теми же эффективными магнитными восприимчивостями, но нормированными на значение эффективной к при 100\% концентрации, то есть при однородном объекте. Сравнение кажущихся и истинных концентраций демонстрирует их близость и доказывает практическую возможность использования эффективных к для оценок содержания полезных ископаемых. Следует отметить, что подобные расчеты проводились при разных величинах $\kappa$ включений и дали аналогичные результаты. Они, в частности, показали принципиальную возможность, зная форму, эффективную $\kappa$ и концентрацию рудных включений некоего изолированного блока, оценить с помощью подобного моделирования магнитную восприимчивость руд, причем с достаточно высокой точностью, и использовать полученные результаты при изучении других блоков данного месторождения.

Обратим теперь внимание на горизонтальные компоненты вектора средней намагниченности $\mathrm{I}_{\mathrm{x}}$. В однородном квадрате $\mathrm{I}_{\mathrm{x}}=0$, и это является естественным следствием симметрии модели относительно вертикальной плоскости, проходящей вдоль простирания. В неоднородных моделях $\mathrm{I}_{\mathrm{x}}$ хотя и мала, но отлична от нуля, а ее величина определяется лишь характером асимметрии распределения включений.

На рис. 30а показаны аномальные поля $\Delta \mathrm{Z}$, создаваемые рассмотренными моделями на разных удалениях от них. Очевидно, вблизи модели неоднородности четко проявляются в поле, тогда как на удалении от верхней кромки, равном стороне квадрата а, они визуально не прослеживаются. 


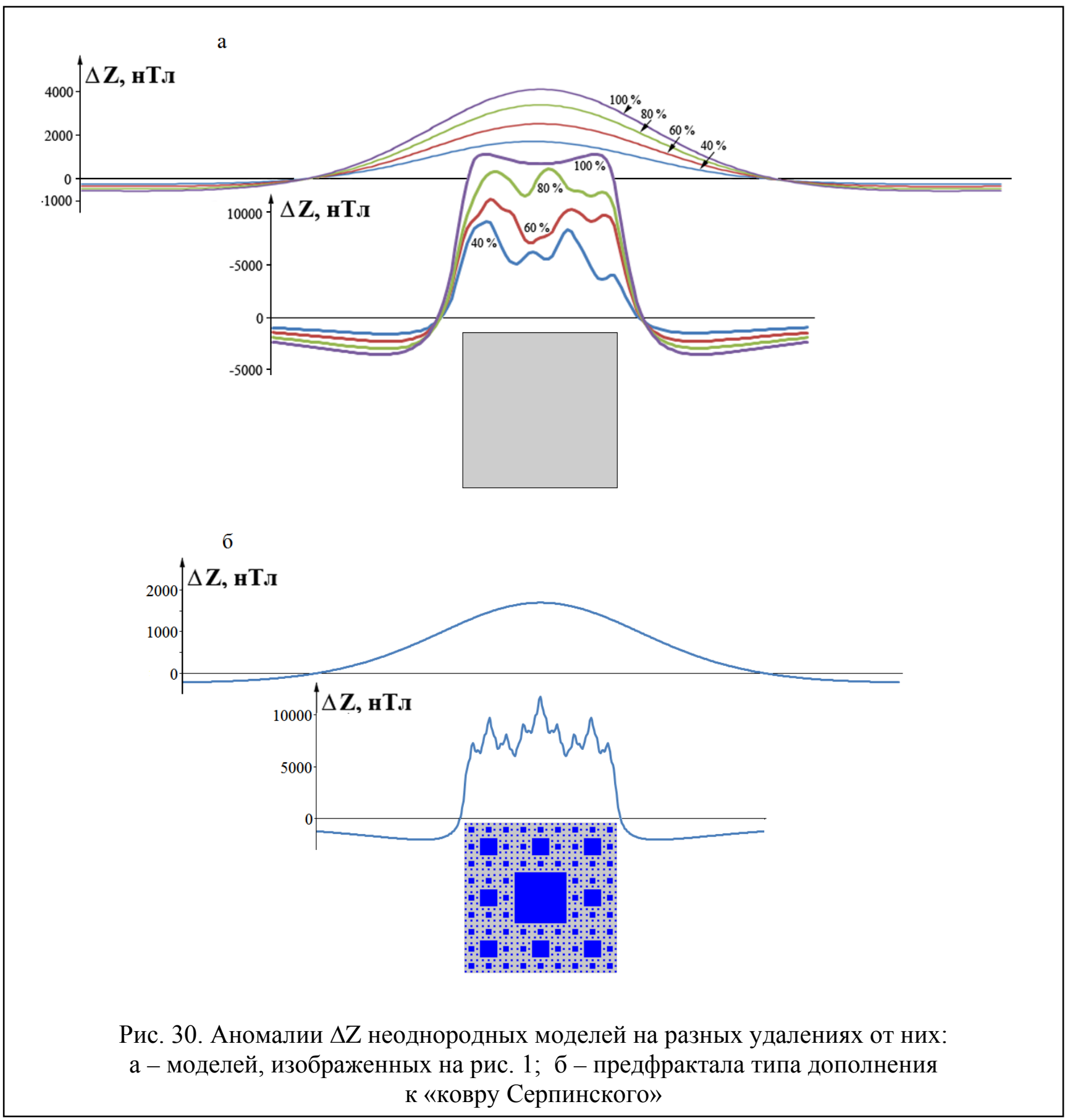

Весьма важным классом неоднородностей являются их упорядоченные и, в первую очередь, фрактальные разновидности. Моделирование показывает, что все отмеченное для моделей со случайным распределением включений распространяется и на них. На рис. $30 б$ представлены аномальные поля $\Delta \mathrm{Z}$ над неоднородной моделью $\mathrm{c}$ аналогичными характеристиками, включения у которой воспроизводят дополнения к «ковру Серпинского» [15]. Здесь также видно, что вблизи модели аномалии четко демонстрируют фрактальный характер неоднородностей, тогда как вдали от нее они визуально не обнаруживаются. Тем не менее, даже на таких относительных удалениях находить проявления неоднородности возможно. 

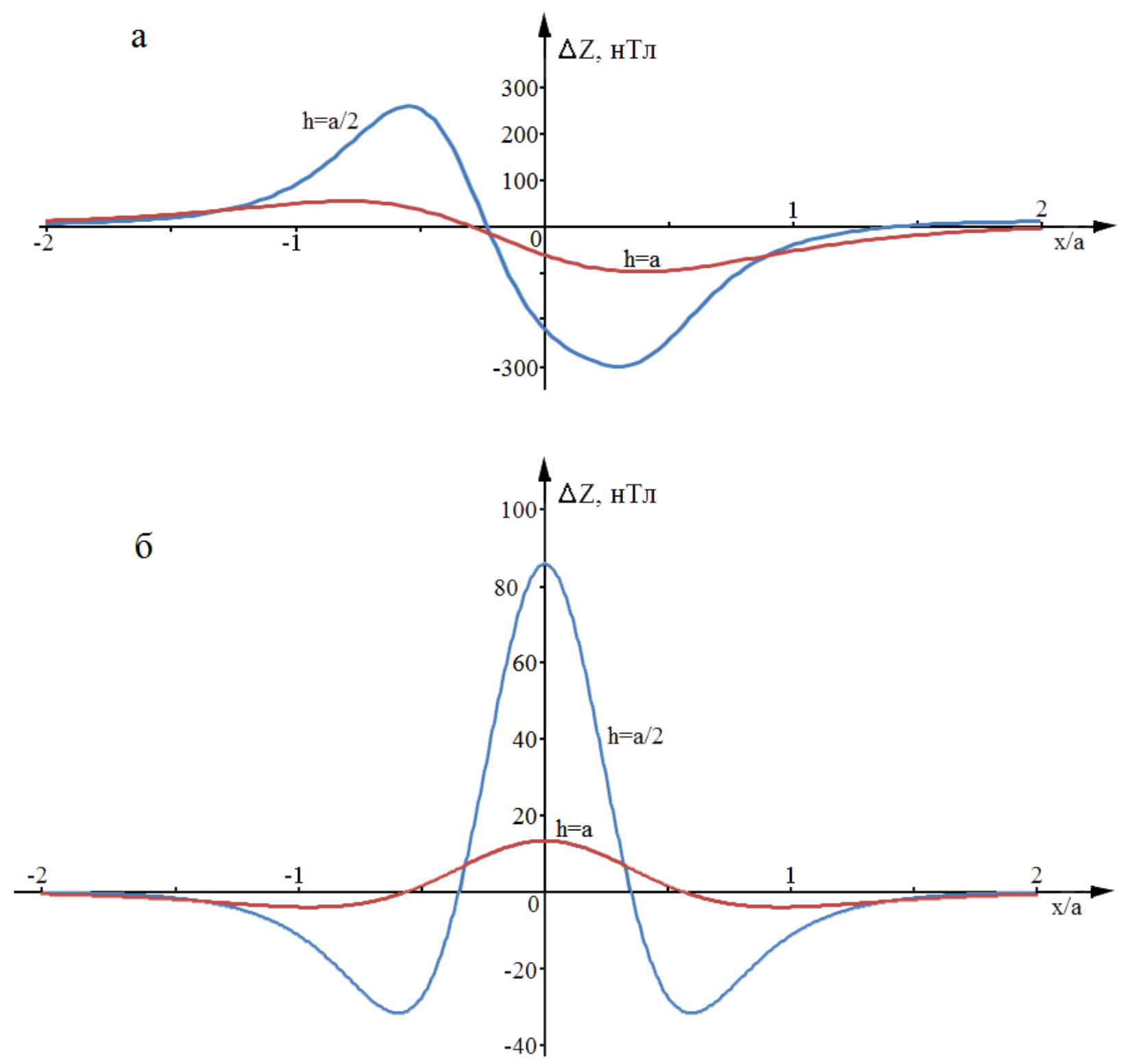

Рис. 31. Магнитные аномалии $\Delta \mathrm{Z}$, связанные с неоднородностями моделей на высотах $\mathrm{h}=\mathrm{a} / 2$ и $\mathrm{h}=\mathrm{a}(\mathrm{a}-$ сторона квадрата): $\mathrm{a}$ - модель со случайным распределением включений при концентрации 40\%; б - предфрактал с фактической концентрацией включений 39,5\%

На рис. 31 показаны те части аномалий $\Delta \mathrm{Z}$, которые связаны именно с неоднородностями двух из рассмотренных моделей на высотах $\mathrm{h}=\mathrm{a} / 2$ и $\mathrm{h}=\mathrm{a}$. На рис. $31 \mathrm{a}$ представлены поля модели со случайным распределением включений $40 \%$ концентрации, а на рис. 316 - поля фрактальной модели. Фактическая объемная концентрация включений в анализируемом предфрактале составляет 39,5\%. Чтобы вычислить их, из аномалий неоднородных моделей вычитались аномалии их однородных аналогов с такими же вертикальными компонентами средней намагниченности.

Хотя эти части аномалий невелики по амплитуде, они при современных точностях съемок в комплексной магниторазведке вполне могут обнаруживаться. Обратим внимание, что на рис. 31a эти аномалии вызваны тем, что размагничивание привело к возникновению у данной модели горизонтальной компоненты вектора средней намагниченности, равной, как следует из таблицы, 0,143 А/м. У однородных моделей в вертикальном намагничивающем поле, характерном для МИП и НЧИМ, в данном случае подобных частей аномалий не могло бы быть. 
Ю.И. Блох

Теоретические основы комплексной магниторазведки

На рис. $3 б$ аномалии имеют совершенно иное происхождение: эта модель строго симметрична, и у ее средней намагниченности нет горизонтальной компоненты, однако здесь четко проявляются следы фрактальной структуры модели. Как видно, неоднородности могут проявляться и на достаточно больших относительных удалениях, где их можно целенаправленно выявлять.

\section{§ 14. Намагничение анизотропных складчато-разрывных структур}

Пласты, изучаемые в комплексной магниторазведке, зачастую образуют складки различной формы, осложненные к тому же разрывными нарушениями. Такие формы характерны для разных сильномагнитных объектов, в том числе, для железистых кварцитов. Исследования, проведенные В.Н. Завойским, продемонстрировали значительное влияние анизотропии магнитной восприимчивости на магнитные аномалии подобных объектов [84], но при проведении расчетов им не учитывалось размагничивание. Изложенные в главе 2 алгоритмы позволяют учитывать этот эффект и анализировать анизотропные складчаторазрывные структур без введения неадекватных упрощений. Рассмотрим некоторые типовые модели.

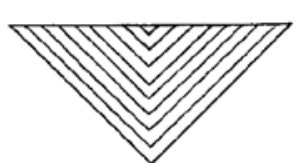

a
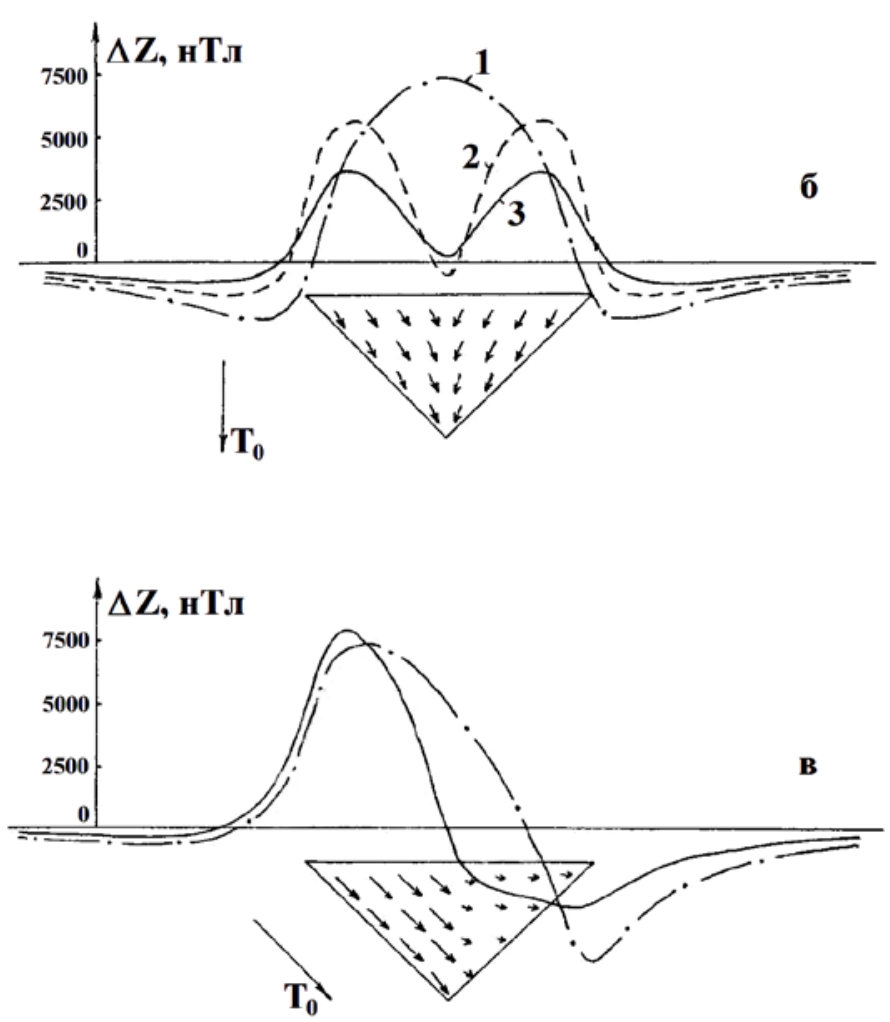

Рис. 32. Намагниченность и магнитные аномалии модели корня пенепленизированной складки с $\kappa_{\mathrm{t}}=1,257 \mathrm{CИ}$ и $\kappa_{\mathrm{n}}=0,251$ СИ: а) поперечное сечение модели и характер ее полосчатости; б) магнитные аномалии модели, намагниченной в однородном вертикальном поле 1 - от изотропной модели при $\kappa=\kappa_{\mathrm{t}}$ с учетом размагничивания, $2-$ от анизотропной модели без учета размагничивания, 3 - с учетом размагничивания; в) магнитные аномалии модели, намагниченной в поле с наклонением $45^{\circ}$ 
На рис. 32а показана двумерная модель корня пенепленизированной складки, подобная рассмотренной В.Н. Завойским без учета размагничивания. Модель представляет собой прямоугольный треугольник с горизонтальной гипотенузой. Шарниром складки является вертикальная линия, перпендикулярная верхней кромке модели. Эта линия делит модель на две части, различающиеся ориентировкой полосчатости, а именно, в каждой из частей полосчатость параллельна соответствующему катету. Компоненты магнитной восприимчивости в обеих частях были выбраны одинаковыми и составляли $\kappa_{\mathrm{t}}=1,257$ СИ $\left(0,1\right.$ СГС) и с $\kappa_{\mathrm{n}}=0,251$ СИ (0,02 СГС). Результаты расчета намагниченности модели в однородном вертикальном поле 50 мкТл, а также магнитная аномалия $\Delta Z$ над моделью приведены на рис. 32б. Для сравнения на том же рисунке показаны графики, вычисленные без учета размагничивания, а также с учетом размагничивания для изотропной модели той же формы, имеющей магнитную восприимчивость 1,257 СИ. Как видно, размагничивание оказывает на аномальное поле модели существенное влияние. Амплитуда максимумов уменьшается под действием размагничивания в 1,5 раза, а значения в центральном минимуме даже противоположны по знаку. Естественно, форма аномалии в анизотропном случае резко отличается от изотропного. На следующем рисунке 32в результаты расчетов для той же модели показаны при наклонном намагничивающем поле. Наклонение поля составляло $45^{\circ}$, то есть в одной из частей модели поле было направлено вдоль полосчатости, а в другой - поперек, что привело к резкой неоднородности намагничения, отражающейся в аномальном поле.

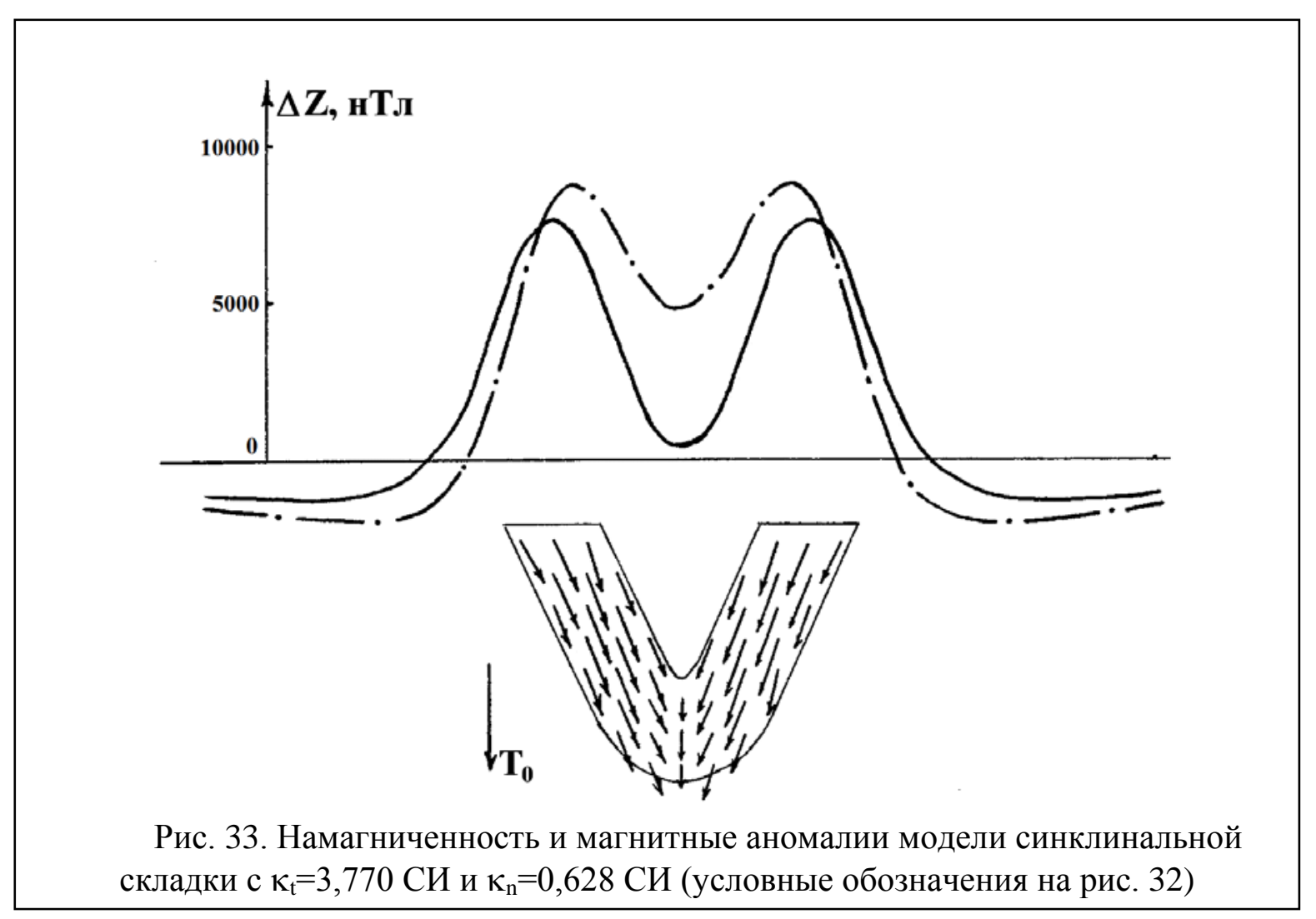

На рис. 33 представлены результаты расчетов для модели синклинальной складки с плавно изменяющимся направлением полосчатости, повторяющим форму самой складки. Главные компоненты тензора магнитной восприимчивости модели в каждой точке считались равными $\kappa_{\mathrm{t}}=3,770$ СИ и $\kappa_{\mathrm{n}}=0,628$ СИ; однородное первичное поле с индукцией 50 мкТл ориентировалось вертикально вниз. Результаты вычислений показывают, что крылья складки при выбранном довольно большом коэффициенте анизотропии магнитной восприимчивости намагничиваются у верхней кромки практически по падению, причем для них справедливы отмеченные закономерности для анизотропных пластов, например, увеличение 
намагниченности по мере удаления от верхней кромки. В замке складки намагниченность резко неоднородна, что связано как с изменением направления полосчатости, так и с влиянием размагничивания. В совокупности эти эффекты значительно изменяют форму графиков аномального поля. Для сопоставления на рис. 33 показана аномалия той же модели с изотропной магнитной восприимчивостью 3,770 СИ.

Рис. 34 содержит результаты вычислений намагниченности и аномального поля более сложной S-образной складки с теми же главными компонентами тензора магнитной восприимчивости. Эта модель под действием анизотропии и размагничивания приобретает резко неоднородную намагниченность, причем средняя часть складки в отличие от крайних намагничивается не по направлению полосчатости, а под некоторым углом к ней. Несмотря на большой коэффициент анизотропии магнитной восприимчивости модели, размагничивание, как видно, может приводить в сложных структурах к появлению существенных компонент намагниченности, перпендикулярных к полосчатости. Интересно отметить, что аномальный график $\Delta \mathrm{Z}$, вычисленный с учетом размагничивания и анизотропии, имеет почти симметричный вид, но совершенно не отражающий симметрию самой модели. Этот факт показывает, что даже при качественной интерпретации магнитных аномалий над анизотропными геологическими объектами необходимо учитывать эффекты, создаваемые размагничиванием.

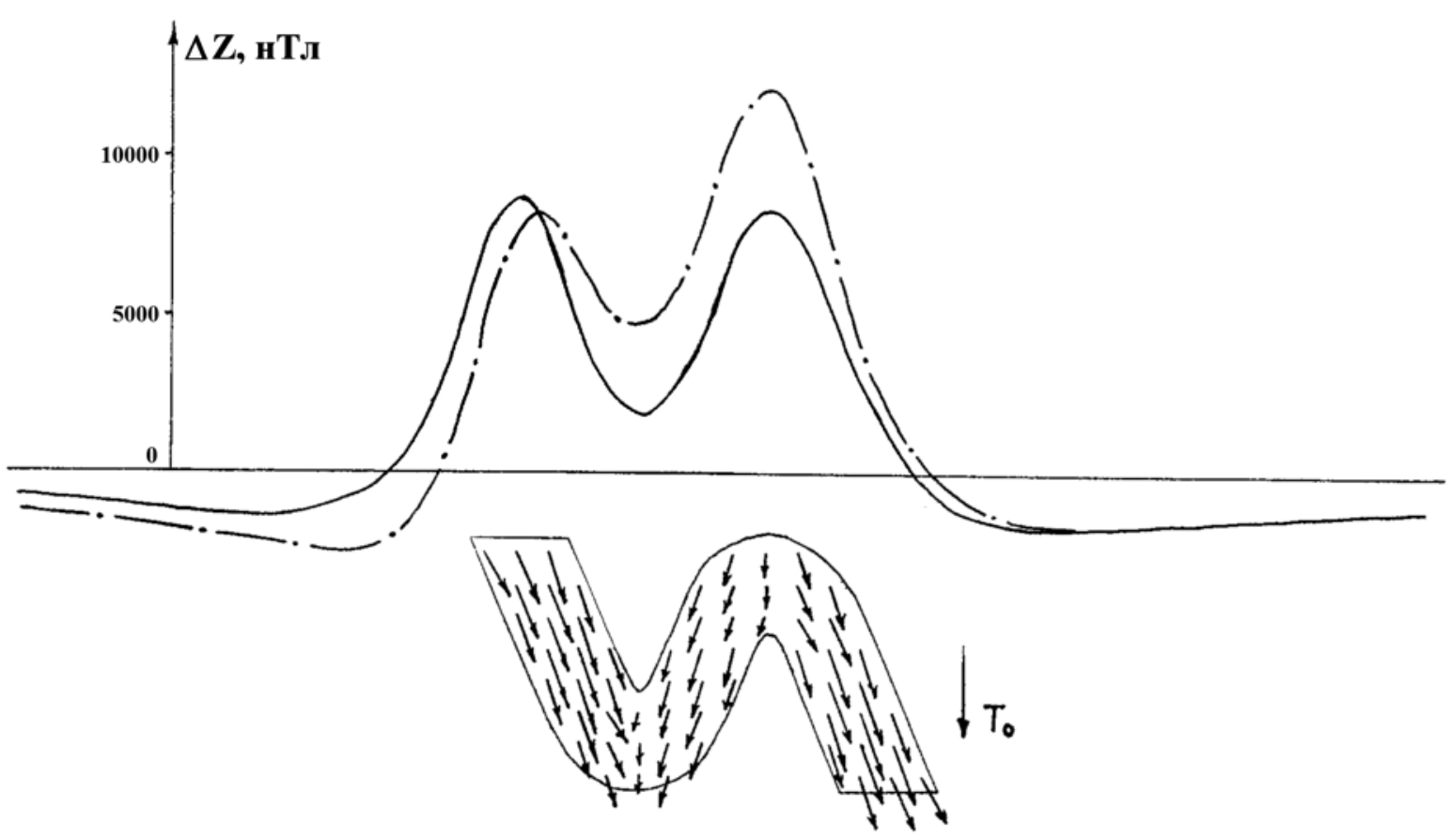

Рис. 34. Намагниченность и магнитные аномалии модели S-образной складки с $\kappa_{\mathrm{t}}=3,770$ СИ и $\kappa_{\mathrm{n}}=0,628$ СИ (условные обозначения на рис. 32)

Предыдущие модели относились к пликативным структурам. Рассмотрим теперь дизъюнктивную структуру: горизонтальный пласт, осложненный сбросом. На рис. 35 показаны графики $\Delta \mathrm{Z}$ модели сброса, характеризующейся следующими главными компонентами тензора магнитной восприимчивости: $\kappa_{\mathrm{t}}=2 \mathrm{CИ}, \kappa_{\mathrm{n}}=1$ СИ. Эти графики получены с учетом и без учета размагничивания и показывают, что его влияние, особенно вблизи самого нарушения, весьма значительно. Оно приводит не только к уменьшению амплитуды аномалии почти вдвое, но и качественно изменяет форму графика, что объясняется резким отклонением вектора намагниченности вблизи плоскости сместителя от направления намагничивающего поля. 


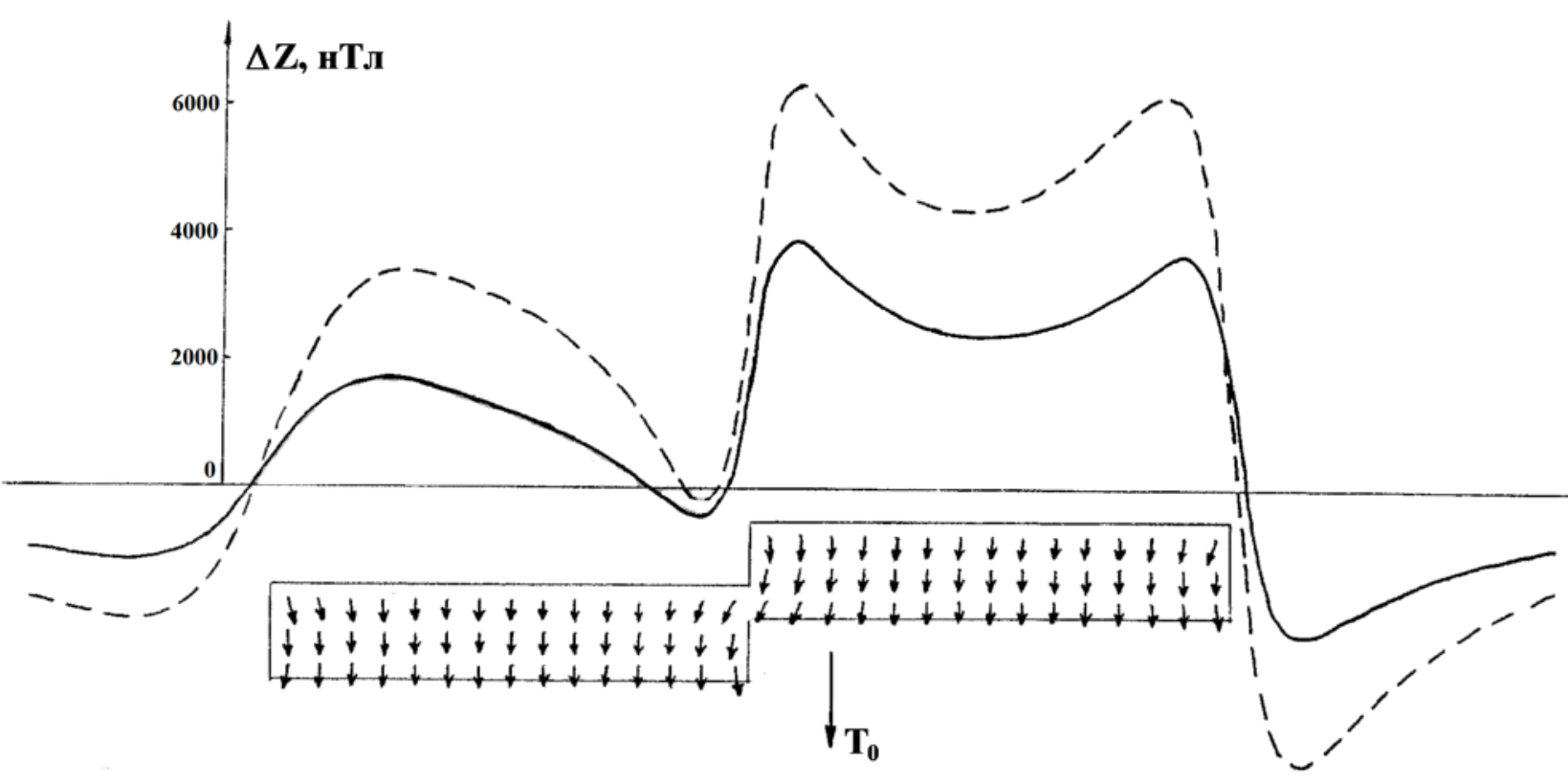

Рис. 35. Намагниченность и магнитные аномалии модели сброса с $\kappa_{\mathrm{t}}=2$ СИ и $\kappa_{\mathrm{n}}=1$ СИ (условные обозначения на рис. 32)

Приведенные данные о намагничении анизотропных складчато-разрывных структур ни в коей мере не могут считаться исчерпывающими, так как геологическое строение различных объектов данного типа весьма разнообразно. Вместе с тем они показывают значительность влияния размагничивания на аномалии сильномагнитных анизотропных структур и возможность его эффективного исследования и учета с помощью разработанных алгоритмов при решении каждой конкретной геологической задачи.

\section{§ 15. Магнитные аномалии, возникающие в результате грозовых разрядов}

Возникновение комплексной магниторазведки в значительной мере было обусловлено тем, что результаты обычной магниторазведки во многих регионах, особенно там, где изучаемые геологические объекты залегают на небольших глубинах, оказывались осложненными чрезвычайно интенсивными помехами. Качественный анализ магнитных аномалий показывал при этом наличие сильной и исключительно неоднородной остаточной намагниченности пород и руд, происхождение которой длительное время оставалось загадкой. Разгадать ее окончательно удалось в середине 90-х годов [49], при этом оказалось, что кажущиеся хаотическими помехи являются вполне закономерными последствиями грозовых разрядов.

Нельзя сказать, что этим вопросом не занимались вовсе. Описывались многочисленные примеры перемагничивания осадочных и изверженных горных пород после удара молнии. Палеомагнитные исследования образцов, отобранных вблизи места грозового разряда, доказывали, что породы под действием молний приобретают нормальную либо идеальную остаточную намагниченность. Тем не менее, проявления грозовых разрядов в магнитных аномалиях систематически не исследовались. В итоге распространенность таких аномалий, другими словами, вероятность возникновения остаточной намагниченности этого типа оценивалась предельно противоречиво: от нескольких процентов до нескольких десятков процентов [49]. Изложенные в главе 2 алгоритмы решения прямых задач дают возможность провести численное моделирование характера магнитных аномалий, возникающих в результате грозовых разрядов в различных физико-геологических условиях.

Изучавшие молнии физики, показали, что основной грозовой разряд проходит по предварительно образованному ионизированному каналу лидера диаметром около 15 см [49]. 
Одиночная молния фактически включает от одного до нескольких десятков разрядов, происходящих через сотые доли секунды, и каждому удару прокладывает путь свой лидер: для первого удара - так называемый «ступенчатый», для последующих - «стреловидный». Наиболее часто: в 80-90\% случаев наблюдается молния, переносящая на землю отрицательный заряд облака. Она в среднем включает 3 главных разряда. Молния, переносящая положительный заряд облака, встречается значительно реже и отличается большим током, увеличенной длительностью импульса и отсутствием повторных разрядов. Результаты изучения морфологии фульгуритов, образующихся в породе после удара молнии, указывают на то, что ток разряда может быть вертикальным, наклонным и даже спиральным. Палеомагнитные исследования отмечали и возможное горизонтальное направление тока молнии вблизи поверхности Земли.

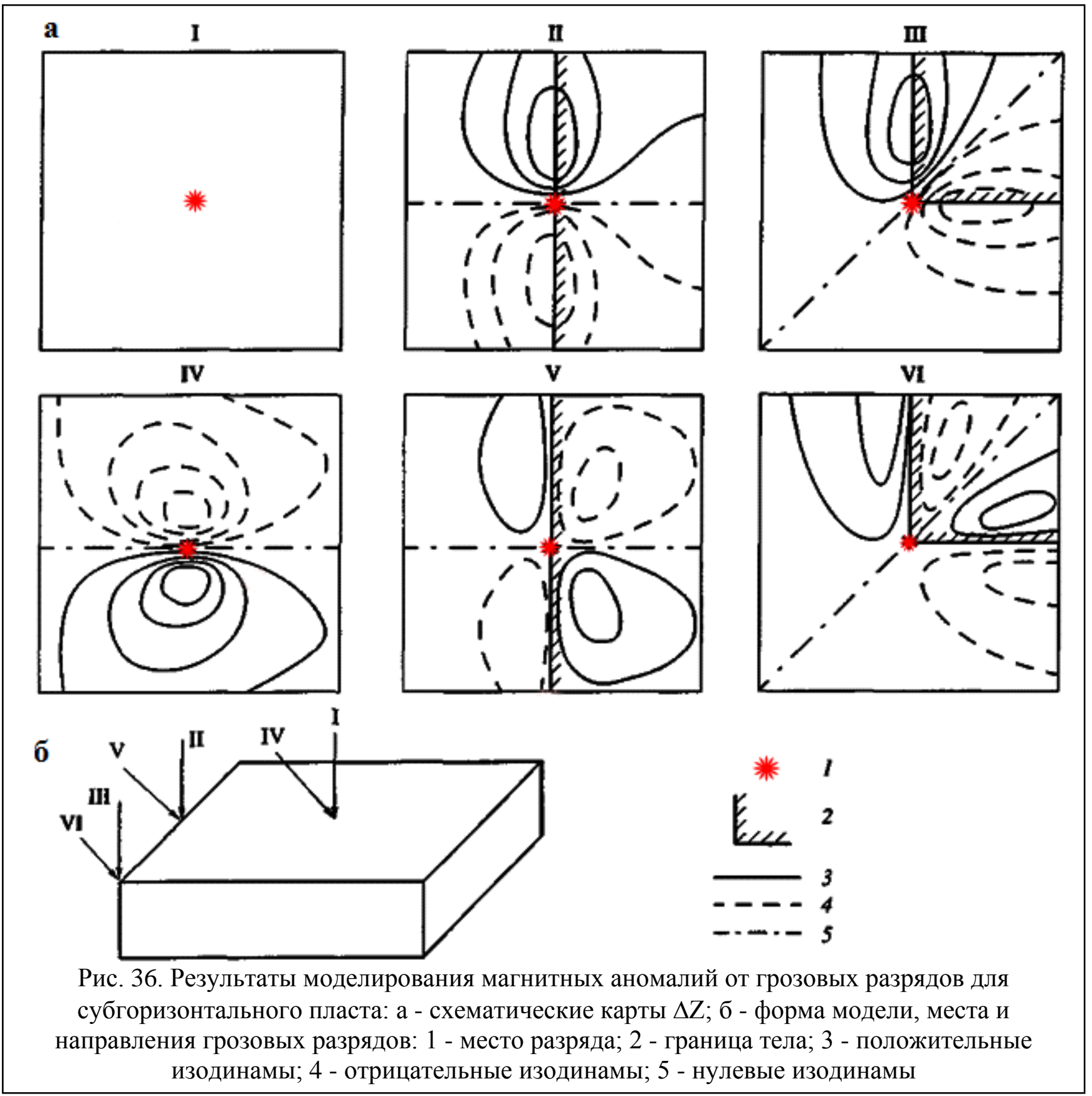

Полученные данные свидетельствуют, что создающий основную часть остаточной намагниченности ток грозового разряда в атмосфере вблизи геологических объектов в большинстве случаев можно считать линейным. Другая часть магнитного поля, влияющего на 
остаточную намагниченность, связана с током растекания в проводящих породах и рудах. Если молния попадает в однородное и изотропное проводящее полупространство, то объемный ток растекания в силу симметрии создает магнитное поле, эквивалентное полю полубесконечного линейного кабеля с током, направленным нормально к поверхности полупространства. Таким образом, намагничивающее поле грозового разряда можно аппроксимировать полем двух сходящихся в точке удара линейных кабелей, один из которых вертикальный, а другой наклонный. Очевидно, неоднородности геоэлектрического разреза по вертикали в месте разряда (проводящие наносы, кора выветривания и т.п.) не исказят данной модели намагничивающего поля. Изменения же проводимости руд по латерали должны оказывать при намагничении менее существенное влияние, нежели латеральные изменения их магнитных свойств.

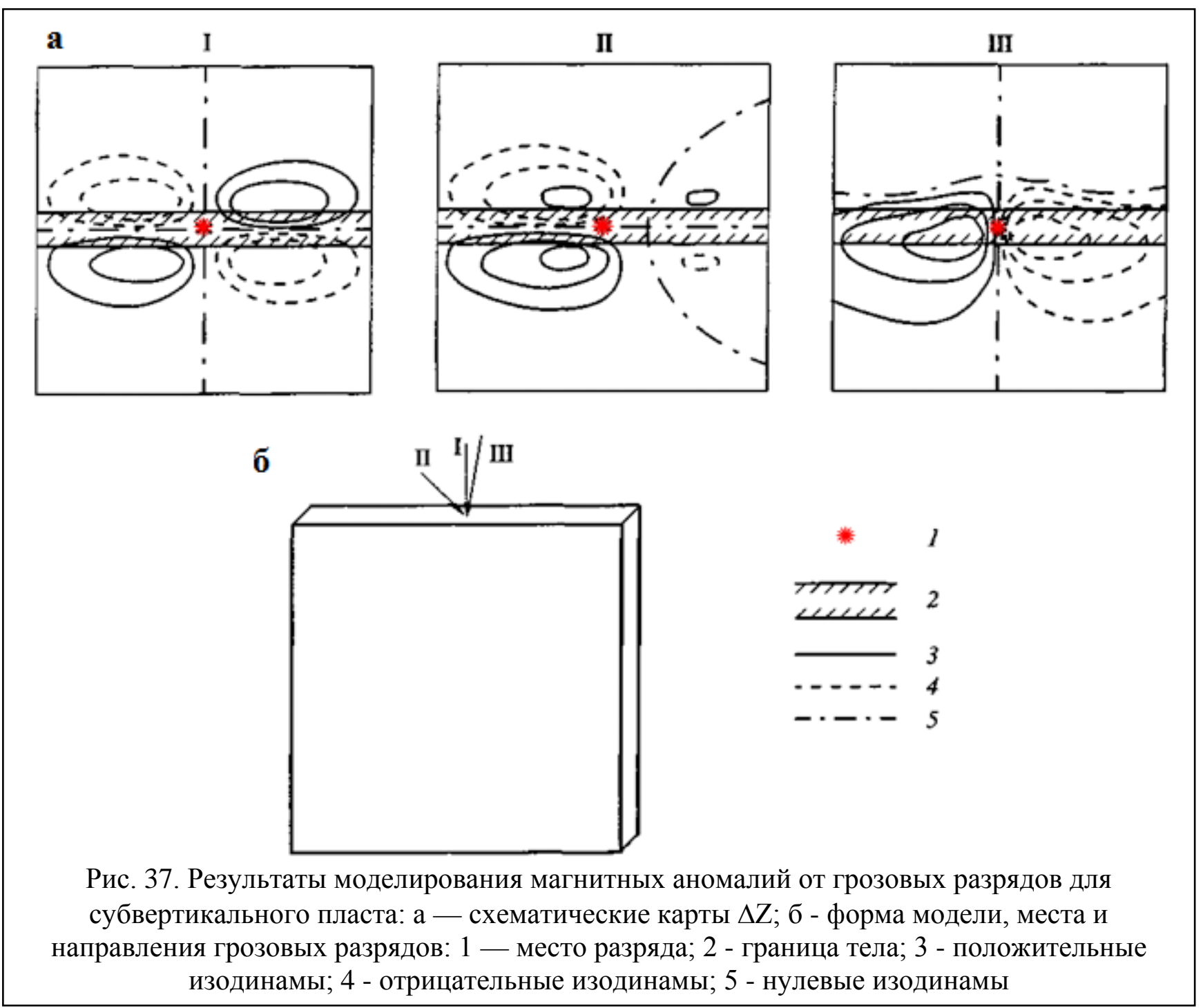

Результаты расчетов, проведенных для двух основных моделей субгоризонтального и субвертикального пластов, представлены на рис. 36 и 37 в виде карт изодинам вертикальной составляющей магнитного поля $\Delta \mathrm{Z}$.

Для понимания характера магнитных аномалий, создаваемых остаточной намагниченностью руд, возникшей вследствие удара молнии, важнейшее значение имеет случай вертикального разряда в однородное полупространство. Магнитное поле такого разряда, вызывающее остаточную намагниченность руд, циркулярно, горизонтально, и направление его в каждой точке определяется известным «правилом буравчика». Возникшая в результате остаточная намагниченность руд должна быть ориентирована так же, а в точках, расположенных на одном расстоянии от аппроксимирующего молнию кабеля, она окажется 
одинаковой по модулю. Такое распределение намагниченности замечательно тем, что не создает внешнего аномального магнитного поля. Это объясняется тем, что любой тонкий тор с осью, совпадающей с линией разряда и вырезанный из намагниченного таким образом полупространства, будет иметь лишь однородную продольную намагниченность, а она не продуцирует аномалии. Это положение иллюстрируется первой из карт на рис. 36, где нет изодинам аномального поля.

Указанный факт имеет несколько важных следствий. Во-первых, из него следует, что аномальное поле, связанное с остаточной намагниченностью руд, приобретенной под действием объемного тока растекания, равно нулю, если грозовой разряд попадает в область, далекую от боковых кромок рудных тел. Это позволяет при изучении аномальных полей практически игнорировать данную часть остаточной намагниченности, тем более что другая ее часть, которая связана с током молний, значительно больше по величине.

Во-вторых, из данного факта следует, что интерпретатору надо весьма осторожно обращаться с данными изучения намагниченности образцов магнетитовых руд. При моделировании магнитного поля магнетитовых месторождений намагниченность весьма часто задают как среднюю по данным измерения на образцах. Если остаточная намагниченность руд содержит часть, связанную с грозовыми разрядами, нормальными к верхней кромке рудных тел, то вследствие данного эффекта наблюденное магнитное поле может оказаться не соответствующим измеренной средней намагниченности руды. Чтобы избежать подобных ошибок, целесообразно в любых случаях привлекать к моделированию данные комплексной магниторазведки.

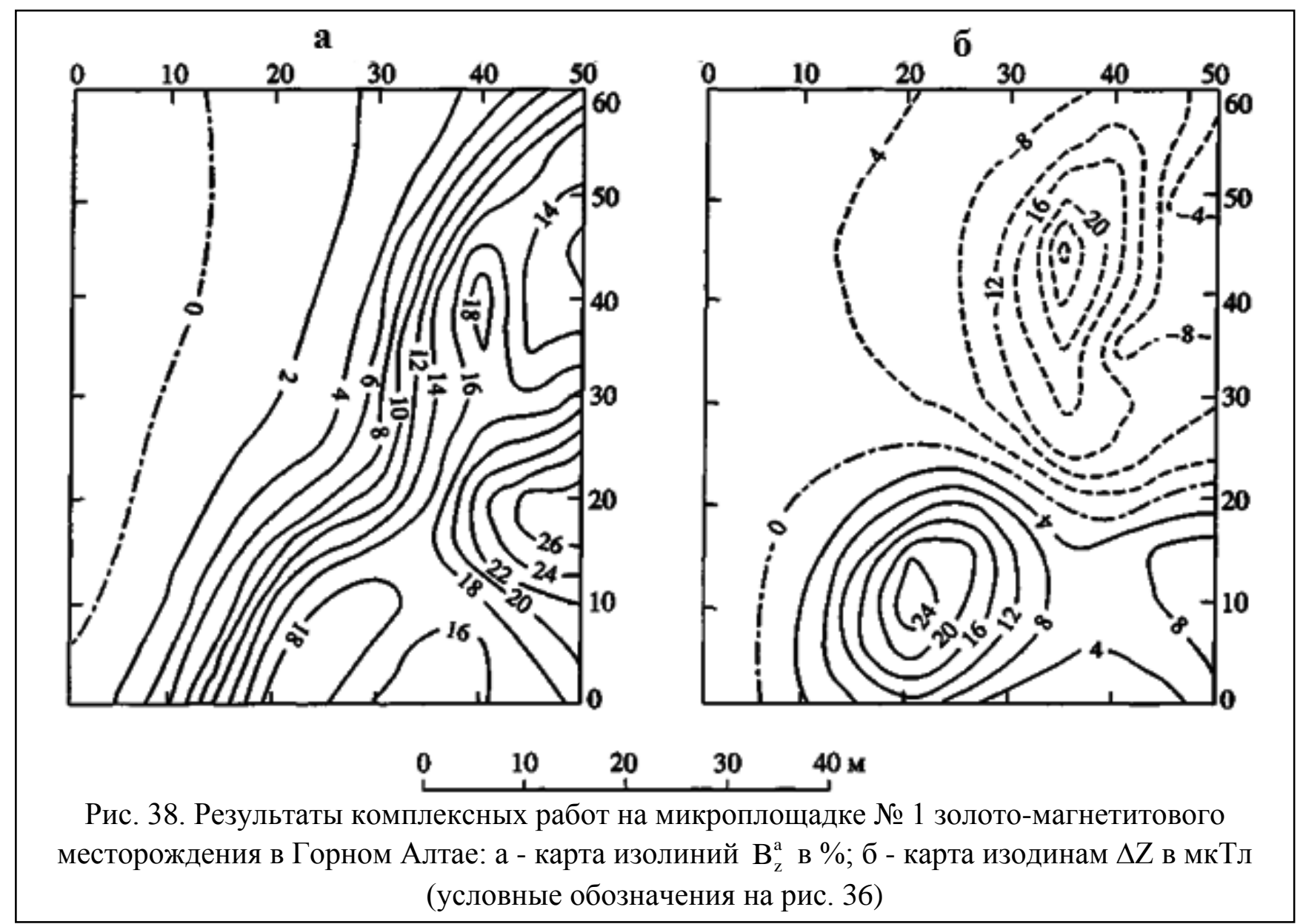

Если грозовой разряд не вертикален, либо попадает в боковую кромку рудного тела, возникает магнитная аномалия, связанная с приобретенной остаточной намагниченностью. Морфология таких аномалий по данным моделирования отражена на рис. 36 и 37. На них 
видно, что под действием грозового разряда могут возникать дополнительные знакопеременные магнитные аномалии дипольного или квадрупольного характера. Эти аномалии накладываются на магнитное поле, связанное с индуктивной намагниченностью руд, что может сильно затруднить геологическую интерпретацию данных магниторазведки.

Результаты математического моделирования показывают качественный характер аномалий, возникающих в результате грозовых разрядов, но не могут указать их амплитуды, размеров в плане и распространенности в природе. Эту информацию с наибольшей надежностью можно получить лишь по данным комплексной магниторазведки на месторождениях магнетитовых руд. Подобные аномалии систематически встречаются на практике, и стоит привести несколько наиболее ярких примеров [49].

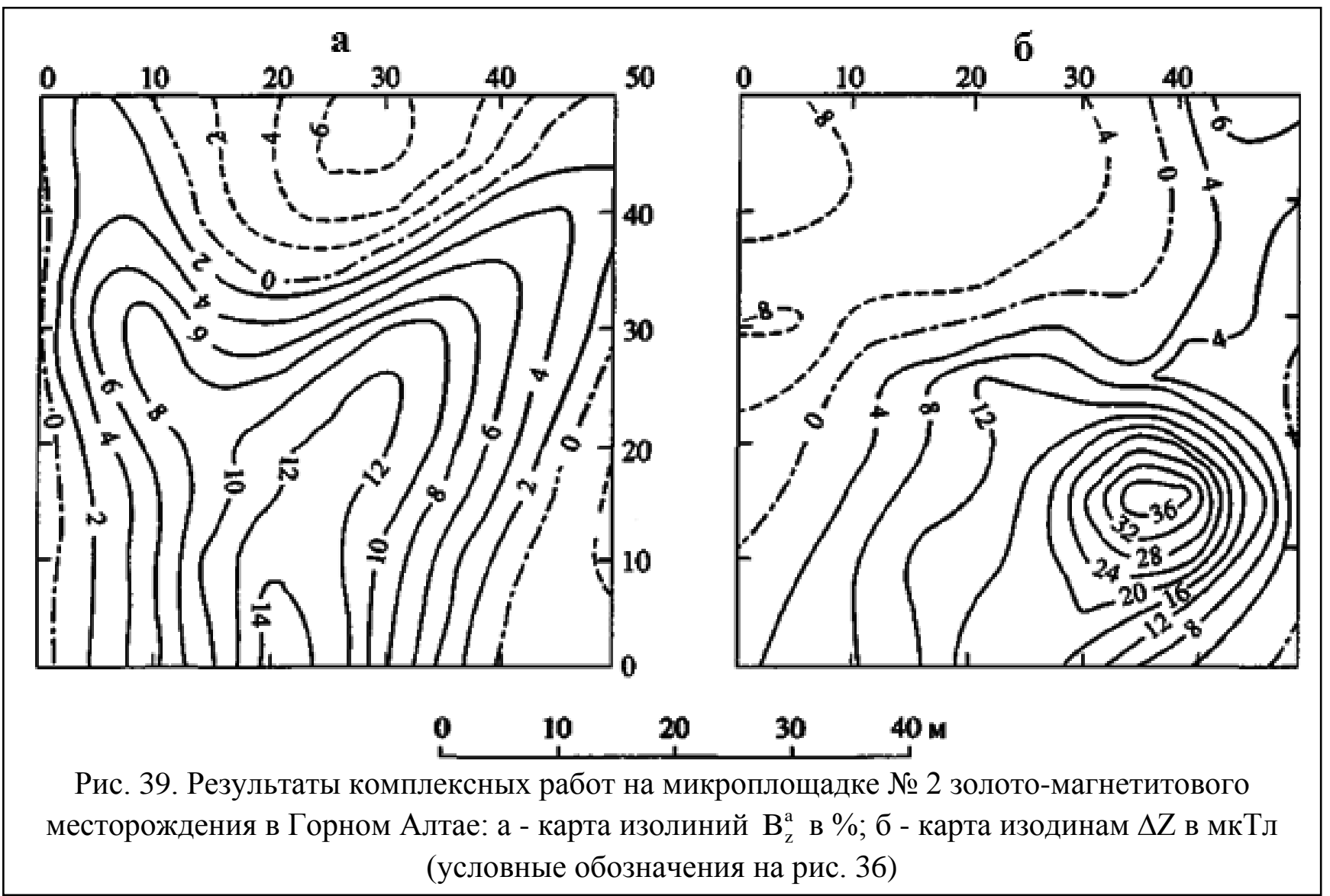

На рис. 38 показаны карты $\Delta \mathrm{Z}$ по данным магниторазведки и $\mathrm{B}_{\mathrm{z}}^{\mathrm{a}}$ по данным метода НП на микроплощадке размерами 50 на 60 м вблизи кромки скарнового золото-магнетитового месторождения в Горном Алтае. Площадка расположена на дне глубокой долины, где руды перекрыты рыхлыми отложениями мощностью около 10 м. В процессе поисковых работ на участке была выявлена сложная магнитная аномалия, которая детализировалась обоими методами по сети $5 \times 5$ м. Геологическое истолкование результатов метода НП достаточно просто. На карте $\mathrm{B}_{z}^{\mathrm{a}}$ по данным метода НП четко выделяется градиентная зона с северовосточным простиранием, приуроченная к боковой кромке субгоризонтальной линзы золотомагнетитовых руд. Мощность линзы небольшая, на что указывает минимум поля $\mathrm{B}_{\mathrm{z}}^{\mathrm{a}}$ на западе микроплощадки. Руда имеет довольно большую магнитную восприимчивость (около 1 СИ), поэтому амплитуда аномалии $\mathrm{B}_{\mathrm{z}}^{\mathrm{a}}$ достигает $26 \%$. В результате магнитной съемки на микроплощадке выявлена знакопеременная аномалия $\Delta \mathrm{Z}$, достигающая в максимуме 27400 нТл, а в минимуме -28700 нТл. Экстремумы $\Delta \mathrm{Z}$ приурочены к градиентной зоне $\mathrm{B}_{\mathrm{z}}^{\mathrm{a}}$. Морфология этой магнитной аномалии, совпадающей с аномалией на второй карте рис. 36, указывает на ее 
вероятное происхождение под влиянием грозового разряда. Молния ударила в кромку рудного тела и, судя по всему, разрушила небольшую его часть, что повлекло за собой искривление изолиний $\mathrm{B}_{\mathrm{z}}^{\mathrm{a}}$ вблизи места разряда в центре микроплощадки.

На рис. 39 показаны аналогичные карты на другой микроплощадке того же месторождения, находящейся в нескольких сотнях метров от предыдущей. Размеры этой площадки 50×50 м. Здесь можно увидеть более сложный случай наложения магнитной аномалии, связанной с грозовым ударом, на достаточно интенсивную аномалию, вызванную индуктивным намагничением руд. Максимум магнитной аномалии, достигающий 40400 нТл, здесь расположен вблизи кромки рудного тела. В то же время минимум на западе площадки фактически наложился на положительную аномалию $\mathrm{B}_{z}^{\mathrm{a}}$ амплитудой около $9 \%$, т.е. на зону положительной магнитной аномалии индуктивного происхождения, поэтому суммарная аномалия здесь невелика и составляет лишь -8300 нТл. Других магнитных аномалий, связанных с грозовыми разрядами на изученной части данного месторождения, не выявлено, что говорит о сравнительно невысокой вероятности появления аномалий данного типа в долинах и на склонах гор.

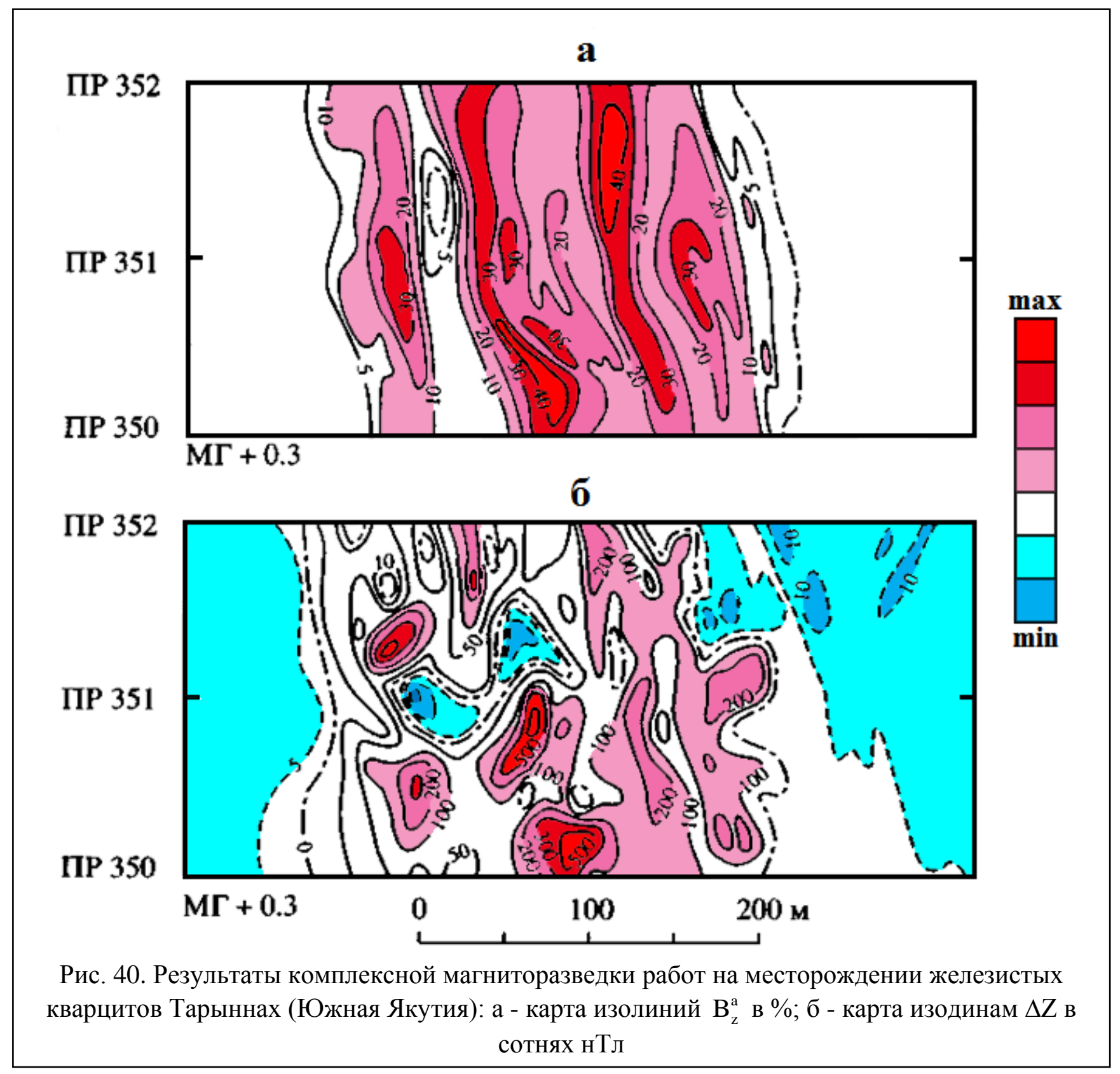


На вершинах гор и на водоразделах вероятность появления подобных аномалий резко возрастает. На рис. 40 приведены фрагменты карт $\Delta \mathrm{Z}$ и $\mathrm{B}_{\mathrm{z}}^{\mathrm{a}}$, полученных в результате съемки масштаба 1:2000 месторождения Тарыннах, расположенного на юге Якутии. Здесь пачки крутопадающих пластов железистых кварцитов приурочены к водоразделу, а их магнитные аномалии сильно искажены за счет остаточной намагниченности, приобретенной рудами под влиянием грозовых разрядов. Наблюдаемые магнитные аномалии по своей морфологии весьма близки к представленным на рис. 37 модельным примерам. Количество локальных аномалий данного происхождения столь велико, что делает практически невозможной геологическую интерпретацию данных традиционной магниторазведки. В то же время данные НЧИМ, свободные от этих влияний, позволяют четко картировать пласты. О приуроченности остаточной намагниченности лишь к приповерхностной части руд данного участка косвенно свидетельствует также и тот факт, что при скважинных исследованиях результаты магниторазведки и НЧИМ здесь практически совпадают [45].

Собранные в различных рудных районах сведения дают возможность сделать следующие основные выводы о параметрах магнитных аномалий, возникающих на магнетитовых месторождениях в результате грозовых разрядов.

1) Грозовые разряды создают на магнетитовых месторождениях дополнительную остаточную намагниченность руд, которая может вызывать изометричные знакопеременные магнитные аномалии амплитудой в несколько десятков тысяч нТл и размерами в плане до 100 M.

2) Магнитные аномалии грозового происхождения достаточно распространены на неглубоко залегающих месторождениях магнетитовых руд. На ровной местности, в долинах и на склонах гор вероятность встретить подобную аномалию составляет первые проценты. Вместе с тем на вершинах гор и на водоразделах эта вероятность возрастает до нескольких десятков процентов. Встречаются участки, где аномалии данного происхождения полностью определяют картину наблюденного магнитного поля.

3) Магнитные аномалии данного происхождения создают значительные помехи при геологической интерпретации результатов обычной магниторазведки. Наиболее кардинальным способом борьбы с этими помехами является применение комплексной магниторазведки. Выявление и исключение аномалий грозового происхождения по ее данным дает возможность извлечения информации о той части остаточной намагниченности магнетитовых руд, которая связана с их генезисом, процессами метаморфизма и т.п. и которая является весьма важной для решения многих геологических задач.

\section{§ 16. Влияние размагничивания на системы параметров источников, однозначно определяемые по аномальному полю}

При интерпретации данных комплексной магниторазведки важнейшее значение имеют две системы параметров, которые по аномальному полю определяются единственно, а именно, система особых точек функций, описывающих аномальные поля, и система гармонических моментов источников [39]. В связи с этим необходимо понимать, как эти параметры определяются по полям, изучаемым в комплексной магниторазведке - в условиях сильного влияния эффекта размагничивания.

Гармонические моменты представляют собой коэффициенты разложения функций, описывающих аномальные поля, в некоторые специальные ряды: для 3D моделей - это ряды по сферическим функциям, для 2D моделей - ряды Лорана. В соответствии с исследованиями, выполненными В.Н. Страховым и автором [181, 182], наиболее наглядно интересующие нас закономерности проявляются при рассмотрении 2D моделей с помощью аппарата теории функций комплексной переменной.

Как известно [39], естественность введения комплексных характеристик вытекает непосредственно из вида дифференциальных уравнений, описывающих магнитное поле. В 
немагнитной среде, где только и могут располагаться реальные точки наблюдений, они могут быть записаны в форме:

$$
\operatorname{rot} \overrightarrow{\mathrm{T}}=0 ; \quad \operatorname{div} \overrightarrow{\mathrm{T}}=0 .
$$

Обозначение Т введено здесь для универсальности, поскольку в такой среде $\overrightarrow{\mathrm{B}}=\mu_{0} \overrightarrow{\mathrm{H}}$, и может, вообще говоря, трактоваться и как напряженность (Н), и как индукция (В). Если горизонтальную компоненту индукции обозначить как X, а вертикальную - как Z, то для двумерных тел (16.1) раскрываются как

$$
\frac{\partial \mathrm{Z}}{\partial \mathrm{x}}-\frac{\partial \mathrm{X}}{\partial \mathrm{z}}=0 ; \quad \frac{\partial \mathrm{X}}{\partial \mathrm{x}}+\frac{\partial \mathrm{Z}}{\partial \mathrm{z}}=0 .
$$

Фактически они представляют собой условия Коши-Римана аналитичности функции

комплексной переменной

$$
\mathrm{T}(\mathrm{u})=\mathrm{Z}(\mathrm{x}, \mathrm{z})+\mathrm{i} \mathrm{X}(\mathrm{x}, \mathrm{z})
$$

$$
\mathrm{u}=\mathrm{x}+\mathrm{i} \mathrm{z} .
$$

Таким образом, дифференциальные уравнения поля совершенно естественно определяют комплексную характеристику, относящиеся к узкому и хорошо изученному классу аналитических функций комплексной переменной. Чаще результаты измерений магнитного поля рассматривают как компоненты или модуль индукции, поэтому функцию Т(u) называют комплексной индукцией магнитного поля. Для 2D модели с поперечным сечением S она описывается следующим интегралом [39]:

$$
T(u)=\frac{\mu_{0} I}{2 \pi} \int_{S} \frac{I(w) d S}{(w-u)^{2}},
$$

где $\mathrm{w}=\xi+\mathrm{i} \zeta-$ комплексная координата текущей точки внутри тела $\mathrm{S}, \mathrm{a}$

$$
\mathrm{I}(\mathrm{w})=\mathrm{I}_{\mathrm{x}}(\xi, \zeta)+\mathrm{i}_{\mathrm{z}}(\xi, \zeta)
$$

комплексная намагниченность. Во всех этих формулах ось х направлена вправо, а ось Z вверх.

Раскладывая (16.5), приходим к следующему ряду Лорана [39]:

$$
\mathrm{T}(\mathrm{u})=\frac{\mu_{0} \mathrm{i}}{2 \pi} \sum_{\mathrm{n}=0}^{\infty} \frac{(\mathrm{n}+1) \mathrm{M}_{\mathrm{n}}\left(\mathrm{u}_{0}\right)}{\left(\mathrm{u}-\mathrm{u}_{0}\right)^{\mathrm{n}+2}},
$$

где

$$
\mathrm{M}_{\mathrm{n}}\left(\mathrm{u}_{0}\right)=\int_{\mathrm{S}} \mathrm{I}(\mathrm{w})\left(\mathrm{w}-\mathrm{u}_{0}\right)^{\mathrm{n}} \mathrm{dS}
$$

комплексный гармонический момент намагниченных масс n-го порядка относительно точки $\mathrm{u}_{0}$. Ряд (16.7) сходится во внешности окружности с центром в точке $\mathrm{u}_{0}$, охватывающей все источники.

Важнейшее значение в практике интерпретации имеют моменты нулевого, первого и второго порядков, так как именно они создают наибольший вклад в аномальное поле и, следовательно, определяются по нему наиболее устойчиво. Из формулы (16.8) следует, что гармонический момент нулевого порядка представляет собой линейный дипольный магнитный момент тела. Для однородно намагниченного объекта он попросту равен произведению комплексной намагниченности на площадь поперечного сечения S, а именно,

$$
\mathrm{M}_{0}=\mathrm{IS} \text {. }
$$

Момент первого порядка характеризует центр намагниченных масс $\mathrm{u}_{c}$ и выражается следующим образом:

$$
\mathrm{M}_{1}\left(\mathrm{u}_{0}\right)=\mathrm{M}_{0}\left(\mathrm{u}_{\mathrm{c}}-\mathrm{u}_{0}\right) \text {. }
$$

Момент второго порядка определяет относительную вытянутость и угол падения вытянутых объектов. В частности, для тонкой пластинки момент второго порядка относительно ее центра $\mathrm{u}_{\mathrm{c}}$ определяется по формуле:

$$
\mathrm{M}_{2}\left(\mathrm{u}_{\mathrm{c}}\right)=\mathrm{M}_{0} \mathrm{l}^{2} \mathrm{e}^{2 \mathrm{i} \varphi}
$$


где 1 - половина длины пластинки, $\varphi$ - угол между ней и горизонтом, отсчитываемый против часовой стрелки от положительного направления оси х. Аналогичные формулы можно получить и для других тел. Так для однородно намагниченного прямоугольного цилиндра со сторонами $2 \mathrm{l}_{\mathrm{a}}$ и $2 \mathrm{l}_{\mathrm{b}}\left(\mathrm{l}_{\mathrm{a}} \geq \mathrm{l}_{\mathrm{b}}\right)$ :

$$
M_{2}\left(u_{c}\right)=M_{0} \frac{l_{a}^{2}-l_{b}^{2}}{3} e^{2 i \varphi},
$$

для эллиптического цилиндра с полуосями $\mathrm{R}_{\mathrm{a}}$ и $\mathrm{R}_{\mathrm{b}}\left(\mathrm{R}_{\mathrm{a}} \geq \mathrm{R}_{\mathrm{b}}\right)$ :

$$
\mathrm{M}_{2}\left(\mathrm{u}_{\mathrm{c}}\right)=\mathrm{M}_{0} \frac{\mathrm{R}_{\mathrm{a}}^{2}-\mathrm{R}_{\mathrm{b}}^{2}}{4} \mathrm{e}^{2 \mathrm{i \varphi} \varphi},
$$

Информацию о гармонических моментах нулевого, первого и второго порядков можно в совокупности наглядно представить в виде так называемого квазиэквивалента, то есть такого тела простой формы, первые моменты которого совпадают с соответствующими моментами истинного объекта [39]. Наиболее часто в качестве квазиэквивалента строят именно пластинки, эллиптические или прямоугольные цилиндры. Отметим, что для построения эллиптических или прямоугольных цилиндров, вообще говоря, требуется априорная информация об их намагниченности; в противном случае можно построить бесконечное семейство тел, каждое из которых обладает свойствами квазиэквивалента. При изучении особенностей влияния размагничивания на гармонические моменты наиболее удобен квазиэквивалент в форме пластинки, построение которого однозначно.

Как было показано в главе 2, в процессе учета размагничивания двумерный объект аппроксимируется совокупностью одинаковых изометричных элементов, поле каждого из которых вычисляется по формуле для линейного диполя. Зная намагниченности элементов и площадь их поперечного сечения, можно рассчитать комплексные гармонические моменты n-го порядка относительно точки u по следующей несложной формуле:

$$
\mathrm{M}_{\mathrm{n}}(\mathrm{u})=\mathrm{s} \sum_{\mathrm{k}=1}^{\mathrm{N}} \mathrm{I}_{\mathrm{k}}\left(\mathrm{u}_{\mathrm{k}}-\mathrm{u}\right)^{\mathrm{n}}
$$

где $\mathrm{s}$ - площадь поперечного сечения каждого из элементов, $\mathrm{I}_{\mathrm{k}}-$ комплексная намагниченность $\mathrm{k}$-го элемента с центром в точке $\mathrm{u}_{\mathrm{k}}, \mathrm{a} \mathrm{N}$ - общее число элементов.

Для исследования влияния размагничивания на гармонические моменты двумерных изотропных объектов была разработана специальная компьютерная программа, с помощью которой производился расчет гармонических моментов нулевого, первого и второго порядков с учетом и без учета неоднородности намагничения, вызванной размагничиванием, а по ним вычислялись параметры квазиэквивалентной пластинки. В случае однородного намагничения комплексная намагниченность определялась по формуле для начального приближения (6.29), то есть с коэффициентом размагничивания кругового цилиндра:

$$
\mathrm{I}=\mathrm{I}_{\mathrm{x}}+\mathrm{i} \mathrm{I}_{\mathrm{z}}=\frac{2 \kappa}{2+\kappa}\left(\mathrm{H}_{\mathrm{x}}^{\text {прв }}+\mathrm{i} \mathrm{H}_{\mathrm{z}}^{\text {прв }}\right) .
$$

При этом моменты всех линейных диполей, представляющих объект, равны. После учета размагничивания они, как было показано в предыдущих разделах, становятся различными как по величине, так и по направлению.

На рис. 41 показаны квазиэквивалентные пластинки некоторых изотропных моделей с магнитной восприимчивостью 6,28 СИ, построенные с помощью разработанной программы. Из центра пластинок исходят векторы нулевого гармонического момента. Результаты свидетельствуют о том, что наиболее существенное влияние размагничивание оказывает на нулевой момент объекта, уменьшая его и отклоняя от направления намагничивающего поля в сторону длинной стороны объекта. Пренебрежение этим влиянием может привести к большим ошибкам при оценке запасов изучаемых месторождений магнитных руд. Дело в том, что, поделив модуль нулевого момента на модуль средней намагниченности, можно в двумерном случае определить площадь поперечного сечения, а в трехмерном - объем исследуемого объекта. Зная плотность руд и коэффициент рудоносности, характеризующий долю 
кондиционных руд, можно затем перейти от определенных по нулевому моменту параметров к запасам месторождения. Сведения о намагниченности получают обычно, проводя лабораторные измерения на изометричных образцах керна или решая линейную обратную задачу [Приложение] на хорошо изученных участках месторождения. При этом, распространяя полученные сведения на другие участки месторождения и тем более на все месторождение в целом, можно допустить ошибку, так как средняя намагниченностть является из-за влияния размагничивания характеристикой объекта, а не вещества, его слагающего. Изменение формы изучаемого объекта влечет за собой изменение намагниченности, поэтому средняя намагниченность месторождения может лишь случайно совпасть с намагниченностью извлеченных образцов или же с намагниченностью одного из участков.
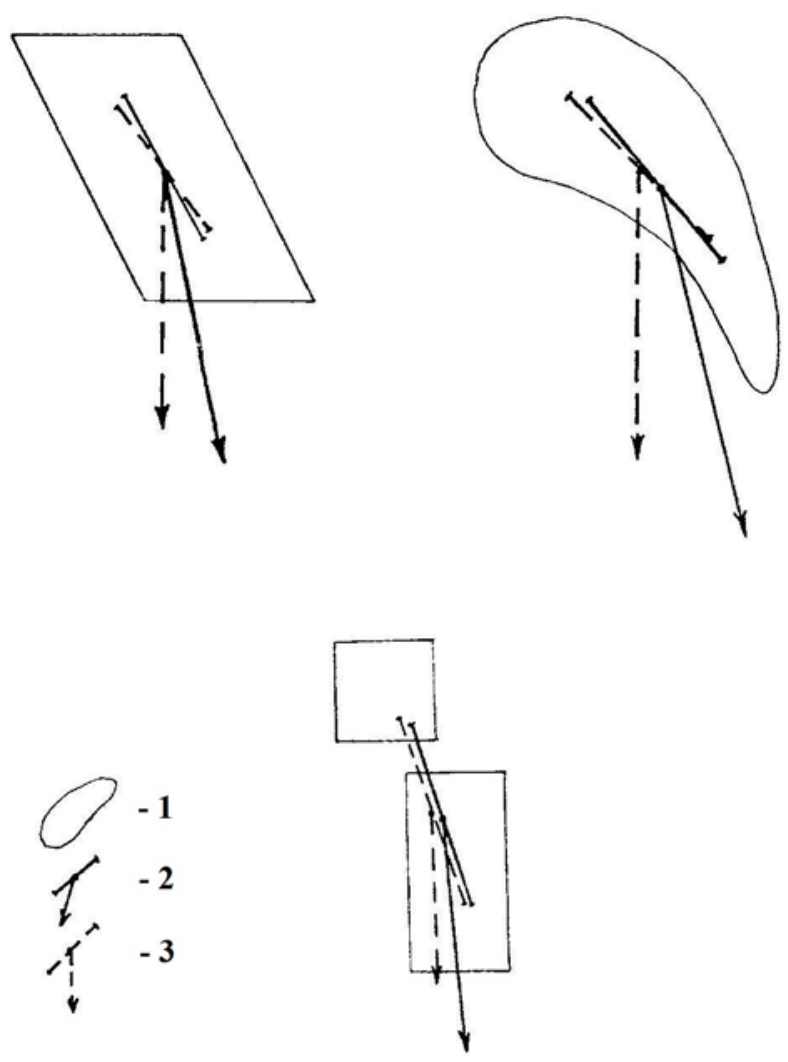

Рис. 41. Влияние размагничивания на результаты определения параметров квазиэквивалентной пластинки: 1 - изучаемый объект, 2 - квазиэквивалентная пластинка и ее магнитный момент при неоднородном намагничении объекта под влиянием размагничивания, 3 - то же при однородном намагничении объекта

Если нулевой момент под влиянием размагничивания изменяется весьма существенно, то отношения первого и второго моментов к нулевому почти нечувствительны к этому влиянию. Действительно, на рис. 41 видно, что параметры квазиэквивалентной пластинки, определяемые данными отношениями, а именно, координаты ее центра, длина и угол падения, в результате размагничивания объекта изменяются лишь незначительно, несмотря на его высокую магнитную восприимчивость. Этот факт дает возможность широкого использования гармонических моментов при интерпретации магнитных аномалий, связанных с сильномагнитными геологическими объектами.

Перейдем теперь к системе особых точек функций, описывающих магнитные аномалии. В рамках традиционной концепции намагничения, благодаря работам В.Н. Страхова, Г.Я. Голиздры, Г.А. Трошкова, А.В. Цирульского и других исследователей, весьма подробно изучен вопрос о связи особых точек с формой намагниченных объектов. Среди двумерных тел 
наиболее важным для практики является класс произвольных многоугольников, поскольку именно к нему относятся наиболее часто встречающиеся тела, в том числе, пласты. Известно, что вершины однородно намагниченных многоугольников совпадают с логарифмическими точками ветвления функции, описывающей комплексную индукцию их аномального магнитного поля, которая имеет следующий вид [39]:

Комплексная напряженность гравитационного поля однородного многоугольника с вершинами в точках $w_{n}$ выражается также полученной в $§ 6$ формулой:

$$
\mathrm{T}(\mathrm{u})=\frac{\mu_{0} \mathrm{I}}{4 \pi} \sum_{\mathrm{n}=1}^{\mathrm{N}} \mathrm{K}_{\mathrm{n}} \ln \left(\mathrm{u}-\mathrm{w}_{\mathrm{n}}\right),
$$

где $\mathrm{w}_{\mathrm{n}}=\xi_{\mathrm{n}}+\mathrm{i} \zeta_{\mathrm{n}}-$ комплексные координаты вершин многоугольника, нумеруемые против часовой стрелки, a $\mathrm{K}_{\mathrm{n}}$ - комплексная постоянная, относящаяся к n-ой вершине и определяемая соотношением

$$
\mathrm{K}_{\mathrm{n}}=\frac{\mathrm{w}_{\mathrm{n}}-\mathrm{w}_{\mathrm{n}-1}}{\mathrm{w}_{\mathrm{n}}-\mathrm{w}_{\mathrm{n}-1}}-\frac{\mathrm{w}_{\mathrm{n}+1}-\mathrm{w}_{\mathrm{n}}}{\mathrm{w}_{\mathrm{n}+1}-\mathrm{w}_{\mathrm{n}}}=2\left(\frac{\xi_{\mathrm{n}}-\xi_{\mathrm{n}-1}}{\mathrm{w}_{\mathrm{n}}-\mathrm{w}_{\mathrm{n}-1}}-\frac{\xi_{\mathrm{n}+1}-\xi_{\mathrm{n}}}{\mathrm{w}_{\mathrm{n}+1}-\mathrm{w}_{\mathrm{n}}}\right) .
$$

В.Н. Страхов показал, что и при намагничении многоугольного цилиндра в поле параллельного кабеля местоположение и тип особенностей остаются теми же [178]. Факт совпадения логарифмических точек ветвления с вершинами однородно намагниченного многоугольника легко продемонстрировать также и на основе физических соображений. Выше говорилось о том, что еще Пуассоном и Грином в начале XIX века была доказана возможность эквивалентной замены магнитного поля однородного объекта полем простого слоя на его поверхности, плотность которого подчиняется интегральному уравнению. Если считать многоугольник однородно намагниченным, то эквивалентный простой слой оказывается на каждой из его сторон однородным, а плотность его определяется как проекция намагниченности на внешнюю нормаль к данной стороне [39]. В связи с этим однородно намагниченный многоугольник оказывается эквивалентным совокупности однородных пластинок, выполненных фиктивными магнитными массами. Края каждой из таких пластинок являются для функции, описывающей аномальное поле, логарифмическими точками ветвления, откуда и вытекает отмеченный результат.

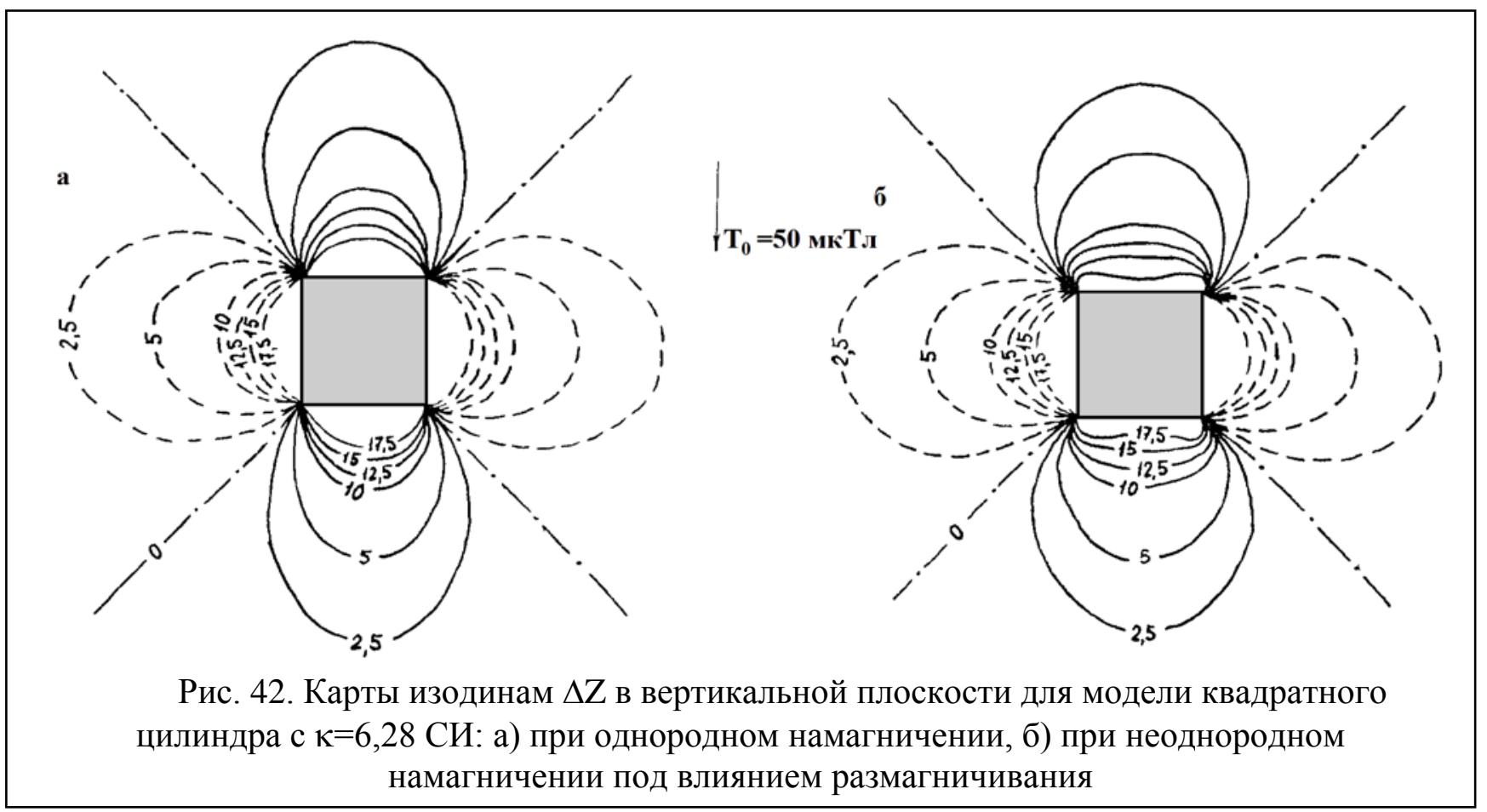


Ю.И. Блох

Теоретические основы комплексной магниторазведки

Сильномагнитные однородные изотропные многоугольники в однородном поле намагничиваются неоднородно, вследствие чего их поле нельзя считать эквивалентным полю совокупности однородных пластинок. Тем не менее, интегральное уравнение Пуассона-Грина и в условиях сильного влияния размагничивания дает возможность построения эквивалентного простого слоя на их поверхности. Более того, из результатов, полученных в $\S 10$, следует, что такой простой слой должен быть непрерывен на всей границе тела, в том числе, и в угловых точках. Отсюда с очевидностью вытекает, что особые точки функции, описывающей индукцию аномального поля сильномагнитного изотропного многоугольника, в условиях влияния размагничивания также совпадают с его вершинами и являются логарифмическими точками ветвления. Этот вывод весьма важен, поскольку дает возможность для широкого класса геологических объектов пользоваться хорошо разработанными методами особых точек даже при высокой магнитной восприимчивости слагающих объекты пород и руд. На рис. 42 и 43 в качестве иллюстрации приведены изодинамы $\Delta \mathrm{Z}$ моделей квадратного цилиндра и мощного пласта в вертикальной плоскости. Как видно из этих рисунков, форма изодинам вблизи особых точек несколько изменяется, но, тем не менее, их локализация по пересечению изолиний и в условиях сильного влияния размагничивания остается вполне эффективной. То же можно сказать и о других способах локализации особых точек.
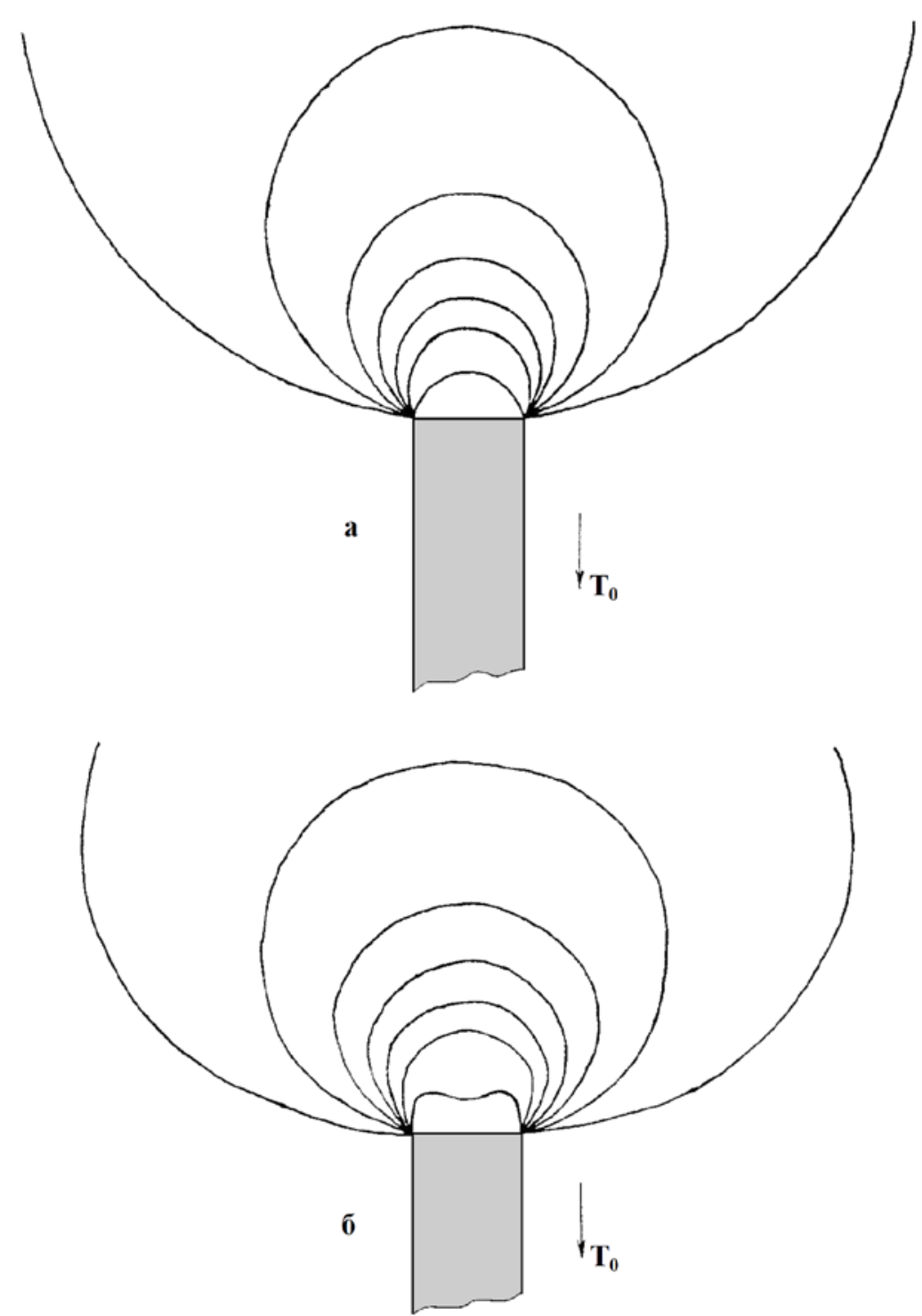

Рис. 43. Карты изодинам $\Delta \mathrm{Z}$ в вертикальной плоскости для модели пласта с $\kappa=2,5$ СИ: а) при однородном намагничении, б) с учетом размагничивания 
Сохранение местоположения и типа особых точек функций, описывающих индукцию аномального поля в условиях влияния размагничивания, характерно не только для многоугольных, но, очевидно, и для круговых, и для эллиптических цилиндров. Для последних фокусы остаются и при влиянии размагничивания алгебраическими полюсами, поскольку эллиптические цилиндры, несмотря на величину их магнитной восприимчивости, однородно намагничиваются в однородном первичном поле. Вместе с тем отмеченная особенность не имеет всеобщего характера. В качестве примера можно рассмотреть тонкий пласт, намагниченный в продольном однородном поле. Традиционная концепция намагничения считает такой пласт однородно намагниченным, вследствие чего его аномальное поле оказывается эквивалентным полю линейной массы на верхней кромке пласта. Отсюда непосредственно следует, что особая точка функции, описывающей индукцию аномального поля тонкого пласта, совпадает со следом его верхней кромки и является полюсом первого порядка. В § 11 было показано, что на самом деле тонкий пласт в однородном продольном поле намагничивается неоднородно: его намагниченность монотонно возрастает от верхней кромки к удаленным от нее частям пласта. В результате аномальное поле, создаваемое им, не равно полю линейной массы на верхней кромке. Как известно [39], плотность фиктивных магнитных масс $\delta_{\mathrm{m}}$ определяется следующим соотношением:

$$
\delta_{\mathrm{m}}=-\operatorname{div} \overline{\mathrm{I}} .
$$

Монотонность изменения намагниченности в тонком пласте означает знакопостоянство эквивалентных ему фиктивных магнитных масс, то есть тонкий пласт, намагниченный в однородном продольном поле, по своему аномальному эффекту эквивалентен неоднородной пластинке, совпадающей с пластом. Плотность фиктивных магнитных масс пластинки, как следует из результатов, полученных в $\S 11$, и из (16.18), монотонно уменьшается по модулю от верхней кромки пласта и на бесконечности стремится к нулю. Отсюда следует, что верхняя кромка тонкого пласта и в условиях сильного влияния размагничивания остается особой точкой функции, описывающей аномальную индукцию, но тип ее меняется. Если в условиях отсутствия размагничивания эта точка является полюсом первого порядка, то при влиянии размагничивания она становится логарифмической точкой ветвления как край неоднородной эквивалентной пластинки. Тем не менее, данная особенность может весьма эффективно локализоваться стандартными методами.

Таким образом, анализ влияния размагничивания на гармонические моменты и особые точки показал, что результаты количественной интерпретации с помощью методов, опирающихся на определение этих параметров, практически незначительно зависят от эффекта размагничивания. В ряде важных практических ситуаций методы моментов и особых точек можно, несмотря на влияние размагничивания, применять для количественной интерпретации так же, как и в рамках традиционной концепции намагничения, пренебрегающей размагничиванием. 


\section{ГЛАВА 4. ТЕОРЕТИЧЕСКИЕ ОСНОВЫ ИНТЕРПРЕТАЦИИ ДАННЫХ КОМПЛЕКСНОЙ МАГНИТОРАЗВЕДКИ}

\section{§ 17. Возможности раздельного определения магнитной восприимчивости и остаточной намагниченности геологических объектов в рамках традиционной магниторазведки}

Приближенная теория намагничения, пренебрегающая адекватным учетом эффекта размагничивания, приводит не только к погрешностям в интерпретации данных традиционной магниторазведки, но и к недооценке еe принципиальных возможностей как метода исследования магнитных свойств горных пород и руд в их естественном залегании. Дело в том, что в рамках этой теории невозможно извлечение из данных наблюдений информации отдельно о магнитной восприимчивости и об остаточной намагниченности геологических объектов. Построенные на ее базе методы интерпретации позволяют делать выводы лишь о характере распределения суммарной намагниченности, естественно, с теми ограничениями, которые накладывает отсутствие единственности и устойчивости решения обратных задач. На самом деле, базируясь на эффектах размагничивания, принципиально возможно раздельно определять магнитную восприимчивость и остаточную намагниченность довольно широкого класса геологических объектов по создаваемым ими магнитным аномалиям.

Физическая основа возможности разделения влияний магнитной восприимчивости и остаточной намагниченности на аномалии земного магнитного поля состоит в следующем:

- эффект размагничивания тел в собственном аномальном поле определяется только их магнитной восприимчивостью и формой;

- естественная остаточная намагниченность тел проявляется в их общей намагниченности аналогично дополнительному намагничивающему полю.

Проиллюстрировать данные положения можно на простейшем примере полупространства, намагниченного в нормальном к нему поле. В соответствии с (11.8) его намагниченность может быть вычислена по следующей формуле

$$
\mathrm{I}=\frac{\kappa \mathrm{H}^{\mathrm{np} \mathrm{B}}+\mathrm{I}_{\mathrm{n}}}{1+\mathrm{N} \kappa}
$$

с коэффициентом размагничивания $\mathrm{N}=0,5$. Элементарное преобразование приводит эту формулу к виду

$$
I=\frac{\kappa\left(H^{\text {прв }}+\frac{I_{\mathrm{n}}}{\kappa}\right)}{1+\mathrm{N} \kappa},
$$

откуда и видно, что остаточная намагниченность проявляется фактически как дополнительное намагничивающее поле $\mathrm{I}_{\mathrm{n}} / \kappa$, а знаменатель в данной формуле, учитывающий размагничивание, от нее никак не зависит.

В отличие от полупространства и эллипсоидов, большинство однородных геологических тел в однородном поле за счет эффекта размагничивания намагничивается неоднородно. Как было показано в предыдущей главе, степень неоднородности намагниченности объекта под действием размагничивания возрастает с ростом его магнитной восприимчивости и заметно отражается в аномальном магнитном поле, измеренном на таких удалениях от объекта, которые не превышают его максимальных линейных размеров. Если форма объекта на основании какойлибо информации известна, то, анализируя в аномалиях проявления неоднородности намагниченности, вызванной размагничиванием, принципиально возможно судить о величине магнитной восприимчивости. Зная же величину к и компоненты вектора $\overline{\mathrm{H}}^{\text {прв }}$, можно учесть их влияние на измеренные аномалии, выделить из них ту часть, которая связана с остаточной намагниченностью, и определить последнюю с учетом размагничивания. 
Среди двумерных объектов, магнитные аномалии которых могут быть истолкованы таким способом, важнейшее значение имеет класс произвольных однородных выпуклых многоугольников. К данному классу, в частности, относится одна из наиболее характерных форм геологических объектов - пласт. Вершины многоугольников являются особыми точками функций, описывающих их магнитные аномалии, и, следовательно, восстанавливаются по аномальному полю однозначно, причем, как было показано в $\S 16$, и для сильномагнитных тел. Для выпуклых многоугольников это влечет за собой единственность определения их формы, что важно для реализации предлагаемого способа при минимальном объеме используемой априорной информации.

Покажем, что магнитная восприимчивость и коллинеарная первичному магнитному полю остаточная намагниченность произвольного известного и однородного по магнитным свойствам тела $\mathrm{V}$ могут быть определены по его магнитной аномалии. Тело $\mathrm{V}$, в каждой точке которого известна намагниченность $\overline{\mathrm{I}}(\mathrm{a})$, создает во внешней точке $\mathrm{t}$ скалярный магнитный потенциал $\mathrm{U}(\mathrm{t})$, определяемый выражением [39]:

$$
\mathrm{U}(\mathrm{t})=-\frac{1}{4 \pi} \int_{\mathrm{V}}\left(\overline{\mathrm{I}}(\mathrm{a}) \operatorname{grad}_{\mathrm{t}} \frac{1}{\mathrm{r}(\mathrm{a}, \mathrm{t})}\right) \mathrm{dV},
$$

где $\mathrm{r}(\mathrm{a}, \mathrm{t})$ - расстояние между точками а и $\mathrm{t}$. Учитывая, что напряженность аномального поля $\overline{\mathrm{H}}$ связана с потенциалом соотношением $\overline{\mathrm{H}}(\mathrm{t})=-\operatorname{grad}_{\mathrm{t}} \mathrm{U}(\mathrm{t})$, а индукция вне тела $\overline{\mathrm{B}}(\mathrm{t})=\mu_{0} \overline{\mathrm{H}}(\mathrm{t})$, можно записать

$$
\overline{\mathrm{B}}(\mathrm{t})=\frac{\mu_{0}}{4 \pi} \operatorname{grad}_{\mathrm{t}} \int_{\mathrm{V}}\left(\overline{\mathrm{I}}(\mathrm{a}) \operatorname{grad}_{\mathrm{t}} \frac{1}{\mathrm{r}(\mathrm{a}, \mathrm{t})}\right) \mathrm{dV},
$$

В свою очередь, как было показано в главе 2, намагниченность тела V подчиняется следующему интегральному уравнению:

$$
\overline{\mathrm{I}}(\mathrm{a})=\kappa \overline{\mathrm{H}}^{\text {прв }}+\mathrm{I}_{\mathrm{n}}+\frac{\kappa}{4 \pi} \operatorname{grad}_{\mathrm{a}} \int_{\mathrm{V}}\left(\overline{\mathrm{I}}(\mathrm{q}) \operatorname{grad}_{\mathrm{a}} \frac{1}{\mathrm{r}(\mathrm{q}, \mathrm{a})}\right) \mathrm{dV},
$$

где q - также точка, принадлежащая телу V. По принятым условиям $\overline{\mathrm{H}}^{\text {прв }}$ и $\overline{\mathrm{I}}_{\mathrm{n}}$ коллинеарны, тогда возможно следующее представление:

$$
\kappa \overline{\mathrm{H}}^{\text {прв }}+\overline{\mathrm{I}}_{\mathrm{n}}=\mathrm{I}_{0} \overline{\mathrm{I}}_{1}
$$

где $\mathrm{I}_{0}$ - модуль данного вектора, а $\overline{\mathrm{I}}_{1}$ - вектор с единичным модулем, направленный по полю $\overline{\mathrm{H}}^{\text {прв }}$. Используя это представление, уравнение (17.4) можно переписать следующим образом:

$$
\frac{\overline{\mathrm{I}}(\mathrm{a})}{\mathrm{I}_{0}}=\overline{\mathrm{I}}_{1}+\frac{\kappa}{4 \pi} \operatorname{grad}_{\mathrm{a}} \int_{\mathrm{V}}\left(\frac{\overline{\mathrm{I}}(\mathrm{q})}{\mathrm{I}_{0}} \operatorname{grad}_{\mathrm{a}} \frac{1}{\mathrm{r}(\mathrm{q}, \mathrm{a})}\right) \mathrm{dV},
$$

Соответственно индукция аномального поля может быть представлена в виде

$$
\overline{\mathrm{B}}(\mathrm{t})=\frac{\mu_{0} \mathrm{I}_{0}}{4 \pi}\left[\operatorname{grad}_{\mathrm{t}} \int_{\mathrm{V}}\left(\overline{\mathrm{I}}_{1} \operatorname{grad}_{\mathrm{t}} \frac{1}{\mathrm{r}(\mathrm{a}, \mathrm{t})}\right) \mathrm{dV}+\operatorname{grad}_{\mathrm{t}} \int_{\mathrm{V}}\left(\frac{\overline{\mathrm{I}}_{\text {неодн }}(\mathrm{a})}{\mathrm{I}_{0}} \operatorname{grad}_{\mathrm{t}} \frac{1}{\mathrm{r}(\mathrm{a}, \mathrm{t})}\right) \mathrm{dV}\right] \text {, }
$$

где $\overline{\mathrm{I}}_{\text {неодн }}(\mathrm{a})=\overline{\mathrm{I}}(\mathrm{a})-\mathrm{I}_{0} \overline{\mathrm{I}}_{1}-$ неоднородная часть намагниченности, подчиняющаяся уравнению (17.6) и пропорциональная величине $\mathrm{I}_{0}$. Характер распределения данной части намагниченности

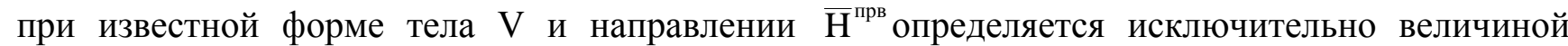
магнитной восприимчивости тела. Таким образом, индукция аномального поля тела V может быть представлена в форме

$$
\mathrm{B}(\mathrm{t})=\mathrm{I}_{0}\left[\overline{\mathrm{B}}_{\text {одн }}(\mathrm{t})+\overline{\mathrm{B}}_{\text {неодн }}(\mathrm{t}, \kappa)\right]
$$

Здесь $\overline{\mathrm{B}}_{\text {одн }}(\mathrm{t})$ - известная и явно заданная функция, определяющая поле, связанное с однородной намагниченностью $\overline{\mathrm{I}}_{1}$ тела $\mathrm{V}$, a $\overline{\mathrm{B}}_{\text {неодн }}(\mathrm{t}, \kappa)$ - неявно заданная функция, определяемая неоднородной частью намагниченности, подчиняющейся уравнению (17.6) и 
зависящая от магнитной восприимчивости тела. Данная структура аномального поля и позволяет раздельно определить величины к и $\mathrm{I}_{0}$.

Пусть известна вертикальная составляющая индукции наблюденного поля $Z_{\text {набл }}$ тела $\mathrm{V}$ в $\mathrm{n}$

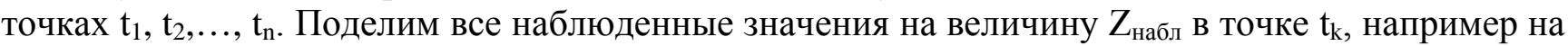
одно из экстремальных значений. Полученные частные не зависят, как следует из (17.8), от $\mathrm{I}_{0}$ и определяются лишь величиной магнитной восприимчивости к, которая может быть найдена методом квазирешений из условия

$$
\sum_{\mathrm{i}=1}^{\mathrm{n}}\left[\frac{\mathrm{Z}_{\text {набл }}\left(\mathrm{t}_{\mathrm{i}}\right)}{\mathrm{Z}_{\text {набл }}\left(\mathrm{t}_{\mathrm{k}}\right)}-\frac{\mathrm{Z}_{\text {одн }}\left(\mathrm{t}_{\mathrm{i}}\right)+\mathrm{Z}_{\text {неодн }}\left(\mathrm{t}_{\mathrm{i}}, \kappa\right)}{\mathrm{Z}_{\text {одн }}\left(\mathrm{t}_{\mathrm{k}}\right)+\mathrm{Z}_{\text {неодн }}\left(\mathrm{t}_{\mathrm{k}}, \kappa\right)}\right]^{2}=\min ,
$$

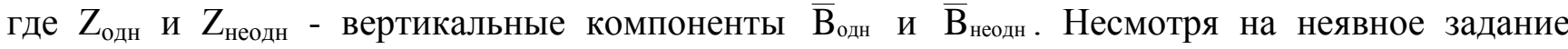
оператора решения прямой задачи с учетом размагничивания, поиск минимума может осуществляться, в частности, путем направленного перебора по к с применением оптимальной стратегии Фибоначчи [39]. Для нахождения минимума в пределах изменения к от 0 до 20 СИ с точностью 0,1 СИ требуется решить интегральное уравнение (17.6) 11 раз. После определения величины магнитной восприимчивости в формуле (17.8) становятся известными и $\mathrm{Z}_{\text {одн }}(\mathrm{t})$ и $\mathrm{Z}_{\text {неодн }}(\mathrm{t}, \kappa)$. Тогда появляется возможность найти $\mathrm{I}_{0}$ из условия

$$
\sum_{\mathrm{i}=1}^{\mathrm{n}}\left\{\mathrm{Z}_{\text {набл }}\left(\mathrm{t}_{\mathrm{i}}\right)-\mathrm{I}_{0}\left[\mathrm{Z}_{\text {одн }}\left(\mathrm{t}_{\mathrm{i}}\right)+\mathrm{Z}_{\text {неодн }}\left(\mathrm{t}_{\mathrm{i}}, \kappa\right)\right]\right\}=\min ,
$$

T.e.

$$
\mathrm{I}_{0}=\frac{\sum_{\mathrm{i}=1}^{\mathrm{n}}\left\{\mathrm{Z}_{\text {набл }}\left(\mathrm{t}_{\mathrm{i}}\right)\left[\mathrm{Z}_{\text {одн }}\left(\mathrm{t}_{\mathrm{i}}\right)+\mathrm{Z}_{\text {неодн }}\left(\mathrm{t}_{\mathrm{i}}, \kappa\right)\right]\right\}}{\sum_{\mathrm{i}=1}^{\mathrm{n}}\left[\mathrm{Z}_{\text {одн }}\left(\mathrm{t}_{\mathrm{i}}\right)+\mathrm{Z}_{\text {неодн }}\left(\mathrm{t}_{\mathrm{i}}, \kappa\right)\right]^{2}}=\mathrm{min},
$$

Так как $\overline{\mathrm{I}}_{1}=\kappa \overline{\mathrm{H}}^{\text {прв }}+\overline{\mathrm{I}}_{\mathrm{n}}$, а $\kappa$ и $\overline{\mathrm{H}}^{\text {прв }}$ известны, легко окончательно найти модуль остаточной намагниченности $\overline{\mathrm{I}}_{\mathrm{n}}$.

Возможности раздельного определения величин $\kappa$ и $\overline{\mathrm{I}}_{\mathrm{n}}$ еще более возрастают в частном случае, когда изучаемый выпуклый многоугольник оказывается симметричным относительно вертикальной оси. В этом случае для реализации данного способа не требуется коллинеарность $\mathrm{I}_{\mathrm{n}}$ и $\mathrm{H}^{\text {прв }}$, так как исходную аномалию $\mathrm{Z}_{\text {набл }}$ можно разделить на четную $\mathrm{Z}_{\text {чет }}$ и нечетную $Z_{\text {нечет }}$ составляющие относительно эпицентра тела. После локализации особых точек и нахождения эпицентра $\mathrm{x}_{0}$ в нем фиксируется начало координат и производится симметризация наблюденной аномалии по формулам

$$
\begin{gathered}
\mathrm{Z}_{\text {чет }}(\mathrm{x})=\mathrm{Z}_{\text {чет }}(-\mathrm{x})=0,5\left[\mathrm{Z}_{\text {наблл }}(\mathrm{x})+\mathrm{Z}_{\text {набл }}(-\mathrm{x})\right], \\
\mathrm{Z}_{\text {нечет }}(\mathrm{x})=\mathrm{Z}_{\text {набл }}(\mathrm{x})-\mathrm{Z}_{\text {чет }}(\mathrm{x}) .
\end{gathered}
$$

Далее по четной составляющей определяется, как было описано выше, магнитная восприимчивость тела $\mathrm{V}$. При известной же величине $\kappa$ компоненты остаточной намагниченности объекта находятся по наблюденной аномалии путем решения линейной обратной задачи, сводящейся к решению системы линейных алгебраических уравнений [Приложение].

Проиллюстрируем возможности предлагаемой методики на примерах. На рис. 44 представлены графики аномалий $\mathrm{Z}$ модели квадратного цилиндра, намагниченного в вертикальном поле, для различных величин магнитной восприимчивости. Значения $Z$ в каждой

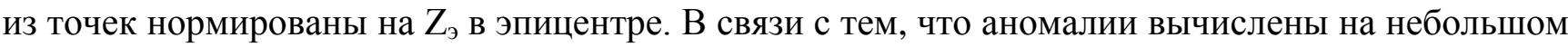
расстоянии от цилиндра, равном 1/7 его стороны, форма графиков меняется с ростом к даже качественно. В этих условиях магнитная восприимчивость теоретически может быть определена с весьма высокой точностью. В реальных условиях, конечно, аномалии, измеренные на небольших удалениях от изучаемого объекта, как правило, осложнены интенсивными помехами, связанными с неоднородностью физических свойств объекта, что ухудшает точность их определения. 
Ю.И. Блох

Наиболее

применение разработанной методики для интерпретации магнитных аномалий над вертикальными пластами. Дело в том, что как раз для пластов неоднородность их намагничения под действием размагничивания проявляется в магнитных аномалиях на достаточно больших расстояниях от их верхней кромки. Там небольшая неоднородность физических свойств не создает значительной помехи. В то же время для многих регионов крутое падение пластов настолько типично, что априорное предположение об их вертикальном падении может быть вполне естественным. На рис. 45 показаны графики $Z$ в долях их экстремального значения для модели вертикального полубесконечного пласта, залегающего на глубине, равной его мощности. Как было показано в $\S 11$, под действием размагничивания верхняя часть пласта вплоть до глубин порядка 10 его мощностей оказывается намагничена слабее, нежели более глубокие части. Эта закономерность отчетливо проявляется в аномалиях, что и позволяет достаточно устойчиво оценивать магнитную восприимчивость вертикальных пластов по их наблюденным или симметризованным аномалиям на довольно больших удалениях от верхней кромки. Отметим, что при неглубоком залегании пласта

соответствующие

графики вблизи эпицентра, как следует из закономерностей намагничения многоугольников, будут иметь вид, аналогичный

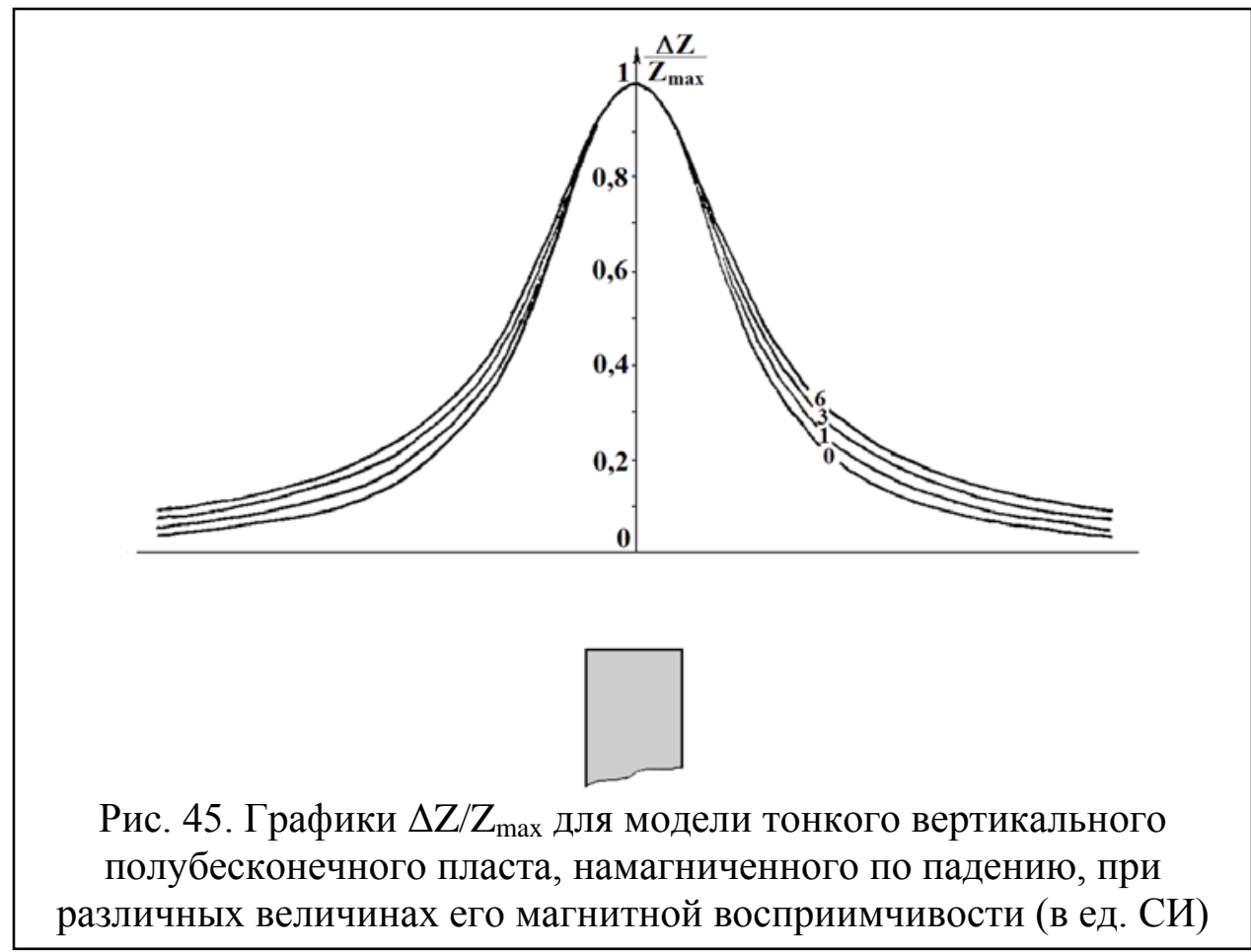
рис. 44 , в то время как боковых минимумов, очевидно, не будет.

При известной глубине залегания верхней кромки пластов уменьшение намагниченности в их верхней части под влиянием размагничивания зачастую ошибочно интерпретируется как проявление мощной зоны окисления. Если же интерпретаторы не пользуются методами особых точек, то, применяя традиционные способы подбора, рассчитанные на однородную 
намагниченность, они систематически завышают реальную глубину. Известно, что глубоко залегающие части пласта оказывают более существенное влияние на форму аномалии в удаленных от эпицентра областях. В связи с этим способы подбора, учитывающие разные области аномалии, дают различные погрешности в определении глубины верхней кромки.

Знание закономерностей искажения дает возможность, сравнивая результаты определения глубины верхней кромки пластов методами особых точек и традиционными методами характерных точек и подбора, судить о величине их магнитной восприимчивости [30].

Обратимся вначале к способу характерных точек для тонких пластов. Как известно, тонким считают такой пласт, мощность которого значительно меньше глубины его залегания. Эта модель перспективна при решении рассматриваемой задачи в связи с тем, что именно на значительном удалении от пласта латеральная неоднородность его состава не оказывает существенного влияния на аномалию, что способствует применению данной методики. У однородно намагниченного по падению вертикального тонкого пласта абсцисса половины максимума аномалии $\Delta \mathrm{Z}$, отсчитанная от эпицентра, равна глубине его верхней кромки [109]. Этот способ часто применяют на практике, в том числе и на месторождениях магнетитовых

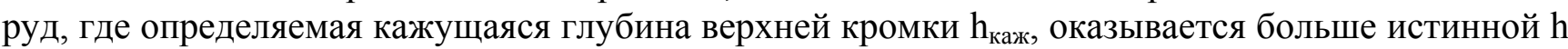
из-за влияния размагничивания. Для выяснения закономерностей заглубления было проведено численное моделирование. Вычисленная на нескольких уровнях аномалия $\Delta \mathrm{Z}$ автоматически интерпретировалась способом «абсциссы половины максимума», а результаты выдавались на печать. На рис. 46 показаны графики получаемых таким образом зависимостей относительного

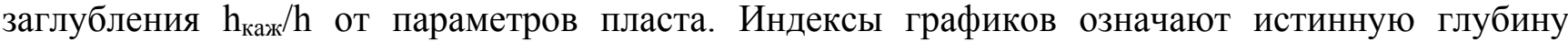
верхней кромки, кратную полумощности пласта - b.

Анализ рис. 46 показывает, что размагничивание может приводить при интерпретации данным способом к систематическому заглублению верхней кромки сильномагнитных пластов на 10-20\%, При небольшой глубине залегания верхней кромки $\mathrm{h}<4 \mathrm{~b}$, когда пласт уже нельзя считать тонким, общая погрешность увеличивается, но доля в ней, связанная с размагничиванием, остается достаточно выдержанной. Необходимо отметить, что практически аналогичные результаты получены и при интерпретации магнитных аномалий способом касательных с коэффициентами для тонкого пласта [109], так как оба способа используют информацию, содержащуюся в

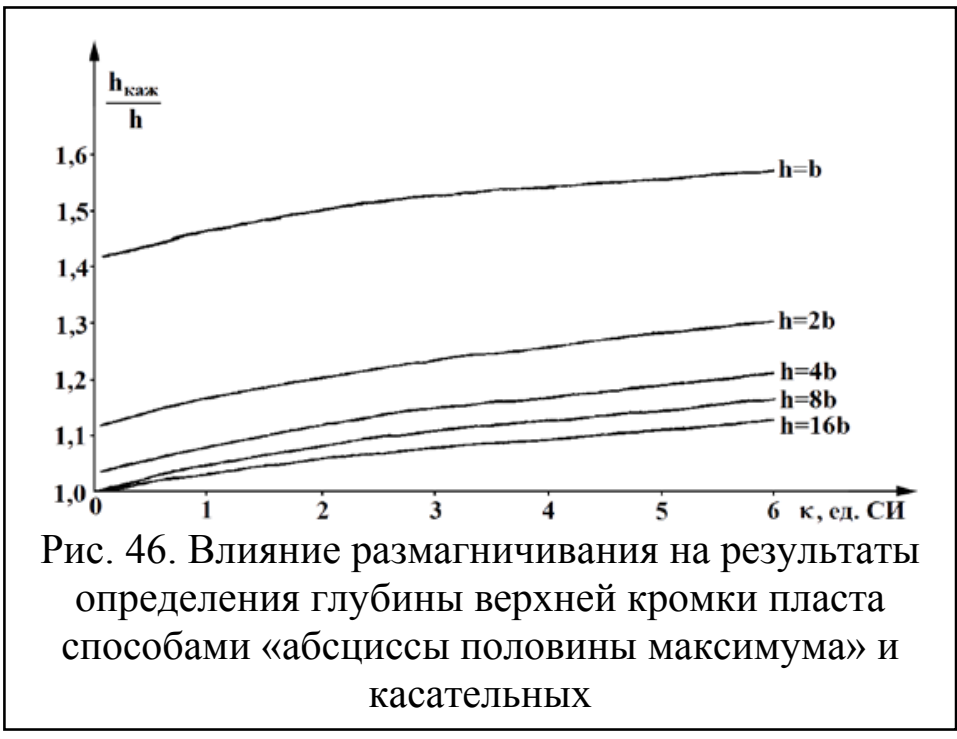
одинаковых, причем небольших участках интерпретируемого графика. Опираясь на данные, изображенные на рис. 46, можно найти магнитную восприимчивость пластов. Наличие естественной остаточной намагниченности не мешает этому, так как характер размагничивания определяется лишь формой тела и величиной к.

Методика определения магнитных свойств сводится к следующему. Вначале любым из хорошо известных методов или их комплексом, например, реализованным в интегрированной системе СИНГУЛЯР [39, 50], локализуются особые точки, характеризующие истинную глубину и мощность пласта. Если интерпретируемый график асимметричен относительно проекции середины верхней кромки на дневную поверхность - точки $\mathrm{x}_{0}$ - производится его

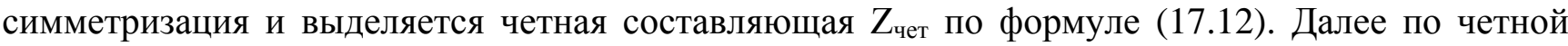
составляющей способами «абсциссы половины максимума» или касательных определяется

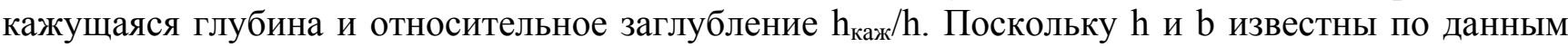
методов особых точек, то, выбрав на рис. 46 соответствующий график, легко найти к, дающую 
такое же относительное заглубление, как и вычисленное. Наконец, зная величину к и индукцию намагничивающего земного поля, можно определить, учитывая размагничивание, компоненты вектора остаточной намагниченности пласта.

Аналогичная методика может быть построена на базе разнообразных модификаций метода подбора. Оказывается, что гипотеза однородного намагничения сильномагнитных пластов приводит при интерпретации методом подбора к наибольшим систематическим ошибкам, если подбор осуществляется по всей кривой, причем безразлично, в какой метрике. Большие систематические искажения глубины дают возможность более устойчивого определения по ним величины магнитной восприимчивости. В связи с этим привлечение в интерпретационный комплекс традиционного подбора по всей кривой наиболее эффективно. Возникновение погрешностей значительных параметров связано с тем, что не существует такого однородно намагниченного пласта, который создавал бы аномалию, идентичную реальной во всех ее областях.

На рис. 47 приведены графики, полученные в результате численного моделирования и характеризующие относительное заглубление верхней кромки пласта при подборе по всей кривой. При проведении эксперимента аномальное магнитное поле вычислялось с у учетом размагничивания на профиле общей длиной $10 \mathrm{~h}$ с шагом $0,2 \mathrm{~h}$. Общее

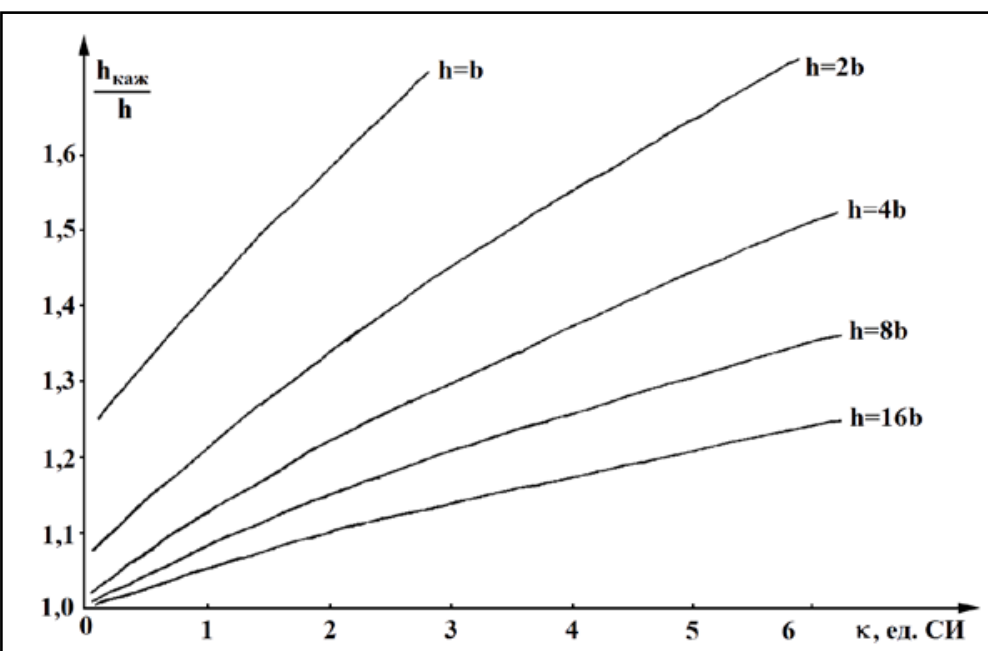

Рис. 47. Влияние размагничивания на результаты определения глубины верхней кромки пласта методом подбора по всей кривой в рамках традиционной концепции намагничения число исходных значений поля составило, таким образом, $\mathrm{n}=51$. Квазирешение обратной задачи искалось путем минимизации следующей функции:

$$
\Phi(\mathrm{h})=\sum_{\mathrm{k}=1}^{\mathrm{n}}\left[\Delta \mathrm{Z}\left(\mathrm{x}_{\mathrm{k}}\right)-\frac{4 \mathrm{~b} \kappa \mathrm{T}_{0} \mathrm{~h}}{\mu_{0}\left(\mathrm{x}_{\mathrm{k}}^{2}+\mathrm{h}^{2}\right)}\right]^{2}=\mathrm{min},
$$

где $\mathrm{T}_{0}$ - модуль индукции вертикального намагничивающего поля. Поскольку функция $Ф(\mathrm{~h})$ является унимодальной, для поиска минимума был применен оптимальный последовательный перебор [39], позволивший за небольшое число проб находить кажущуюся глубину с точностью 1\%. Естественно, изменяя условия подбора: длину интерпретационного профиля, шаг наблюдений и т.П., мы будем получать различные кажущиеся глубины. Тем не менее, рис. 47 иллюстрирует общий характер систематических искажений, возникающих при подборе в рамках традиционной теории намагничения. При использовании подобных графиков для определения природы магнитных аномалий, естественно, необходимо строго выдерживать соответствие условий подбора теоретических и практических данных.

Продемонстрируем некоторые возможности предлагаемого подхода на примере одного из хорошо изученных профилей Горкитского месторождения, относящегося к Ималыкскому железорудному району Южной Якутии. По геологическим данным на месторождении выделяются два структурных яруса: нижний протогеосинклинальный и верхний платформенный. Мощность верхнего яруса, сложенного терригенными и карбонатными породами верхнего протерозоя и четвертичными отложениями, колеблется от 35 до 80 м. В строении нижнего яруса принимают участие глубоко метаморфизовапные породы верхнего архея: метаультрабазиты, граниты и мигматиты, где располагаются пласты железистых кварцитов, объединенные в крутопадающие пачки. Вертикальное падение пластов, а также их 
Ю.И. Блох

вытянутость в плоскости меридиана на несколько километров дают возможность проводить интерпретацию на основе 2D модели. При этом характер графика наблюдаемой аномалии дает право утверждать, что и вектор остаточной намагниченности руд направлен практически по падению пласта.

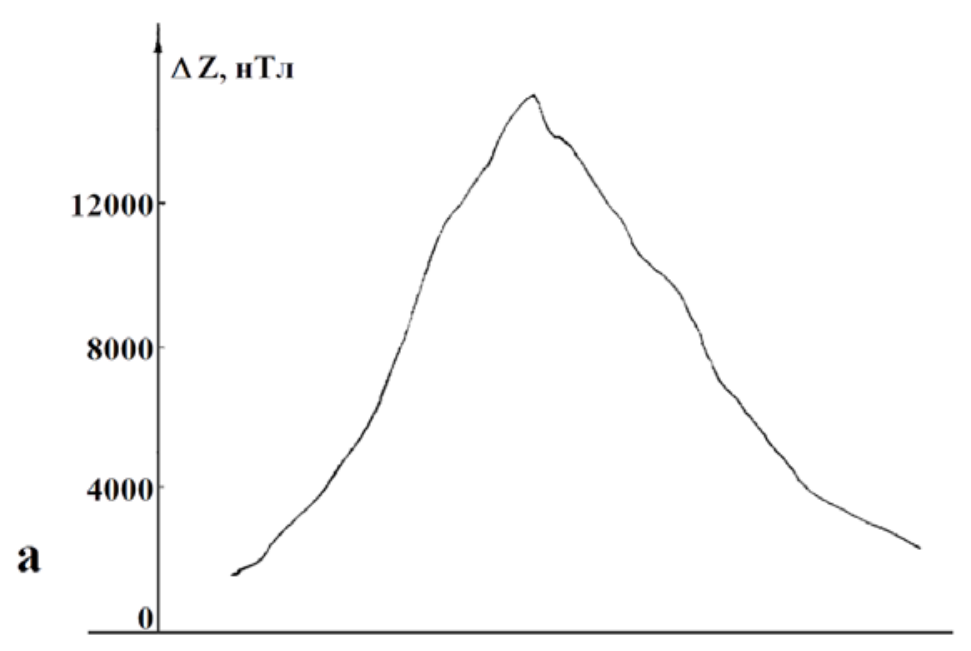

б

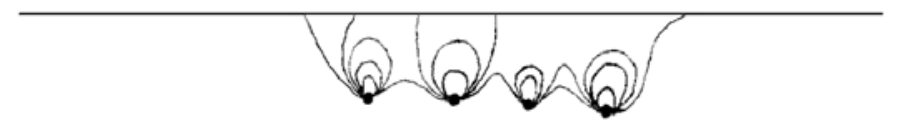

3

B

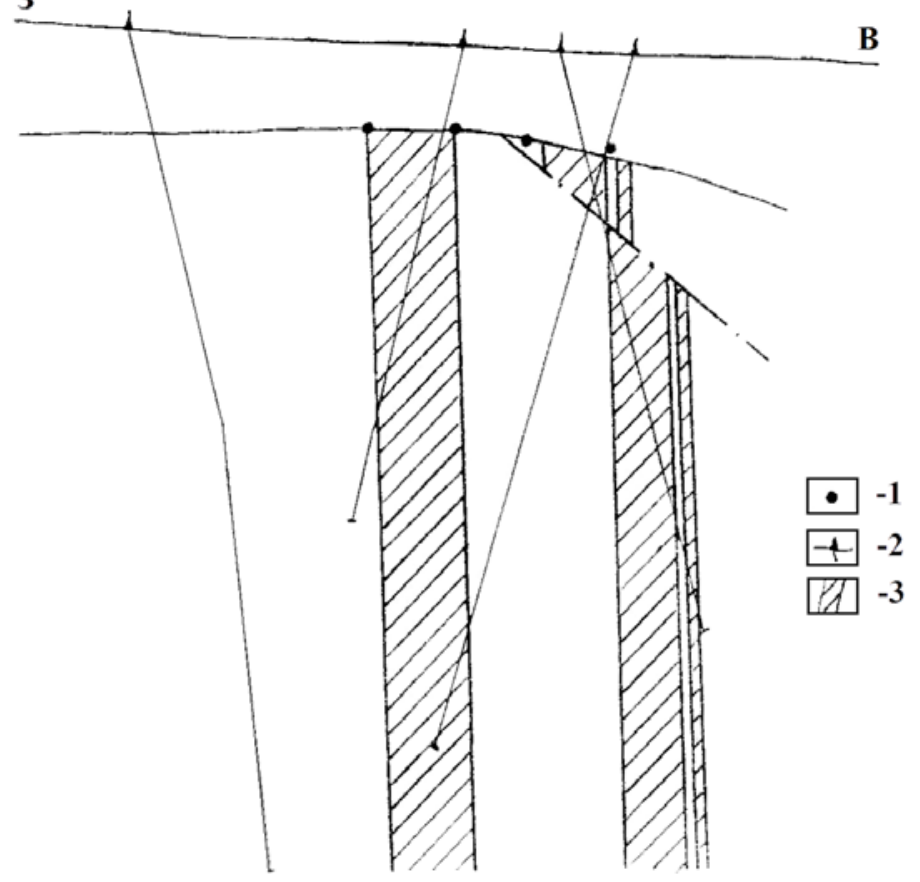

Рис. 48. Результаты локализации особых точек на одном из профилей Горкитского месторождения: а - наблюденный график $\Delta \mathrm{Z}$; б - изолинии $\partial \mathrm{Z} / \partial \mathrm{z}$ в вертикальной плоскости и особые точки, в - схематический геологический разрез (по М.Б. Гребню и А.М. Сафонову) и локализованные особые точки: 1 - местоположение особых точек, 2 - скважины, 3 -железистые кварциты

На первом этапе интерпретации были определены особые точки с помощью разработанной В.Н. Страховым методики аналитического (аппроксимационного) продолжения в горизонтальный слой [39]. Поле $\Delta \mathrm{Z}$, наблюденное с шагом 10 м, для усиления локализации было пересчитано в вертикальный градиент $\partial \mathrm{Z} / \partial \mathrm{z}$ и продолжено по стандартной методике вниз 
на четыре уровня с глубинами 10, 20. 30 и 40 м. Построенные по этим данным изолинии в вертикальной плоскости экстраполированы до пересечения в особых точках, как показано на рис. 48, б. На нем видно, что на глубине около 60 м имеются четыре особые точки. Полученное поведение изолиний вертикального градиента магнитного поля близ особенностей отвечает тому их типу, который характерен для угловых точек пластов. Локализованные таким образом особые точки сопоставлены с имеющимся геологическим разрезом (рис. 48, в). Как видно из рисунка, результаты, полученные методом особых точек, хорошо согласуются с данными бурения, особенно для западной пачки. Для восточной пачки, осложненной субгоризонтальным нарушением, результаты несколько хуже, но и они не противоречат геологическим данным и отличаются от них менее чем на шаг наблюдений. Отметим еще раз, что при локализации особых точек никакой априорной информации не требуется. В итоге оказалось, что глубина западной пачки составляет 60 м, а ее мощность равна глубине, т. e. h=2b.

На втором этапе по левой ветви графика $\Delta \mathrm{Z}$ с помощью способа касательных была определена кажущаяся глубина, оказавшаяся равной 73 м, т. е. относительное заглубление составило 73/60=1,21. Проведя мысленно на рис. 46 прямую, параллельную оси абсцисс, через значение 1,21 и найдя ее пересечение с графиком, отвечающим соотношению $\mathrm{h}=2 \mathrm{~b}$, получаем $\kappa=2,1$ ед. СИ. Для левой ветви все значения $\Delta \mathrm{Z}$ были нормированы на значение над серединой верхней кромки западной пачки, исправленное за влияние соседней пачки. Результаты были наложены на семейство графиков $\Delta \mathrm{Z} / \mathrm{Z}_{\max }$, рассчитанное с учетом размагничивания для модели вертикального пласта с тем же отношением $\mathrm{b} / \mathrm{h} \quad$ при различных к. Нормированные наблюденные данные расположились, как показано на рис. 49. С помощью интерполяции легко установить, что магнитная восприимчивость пласта около 2 СИ, то есть совпадает с результатом, полученным с применением способа касательных. Остаточная намагниченность руд, оцененная на основе полученной информации, составила $12 \quad \mathrm{~A} / \mathrm{M}$. Определенные таким образом значения магнитных свойств
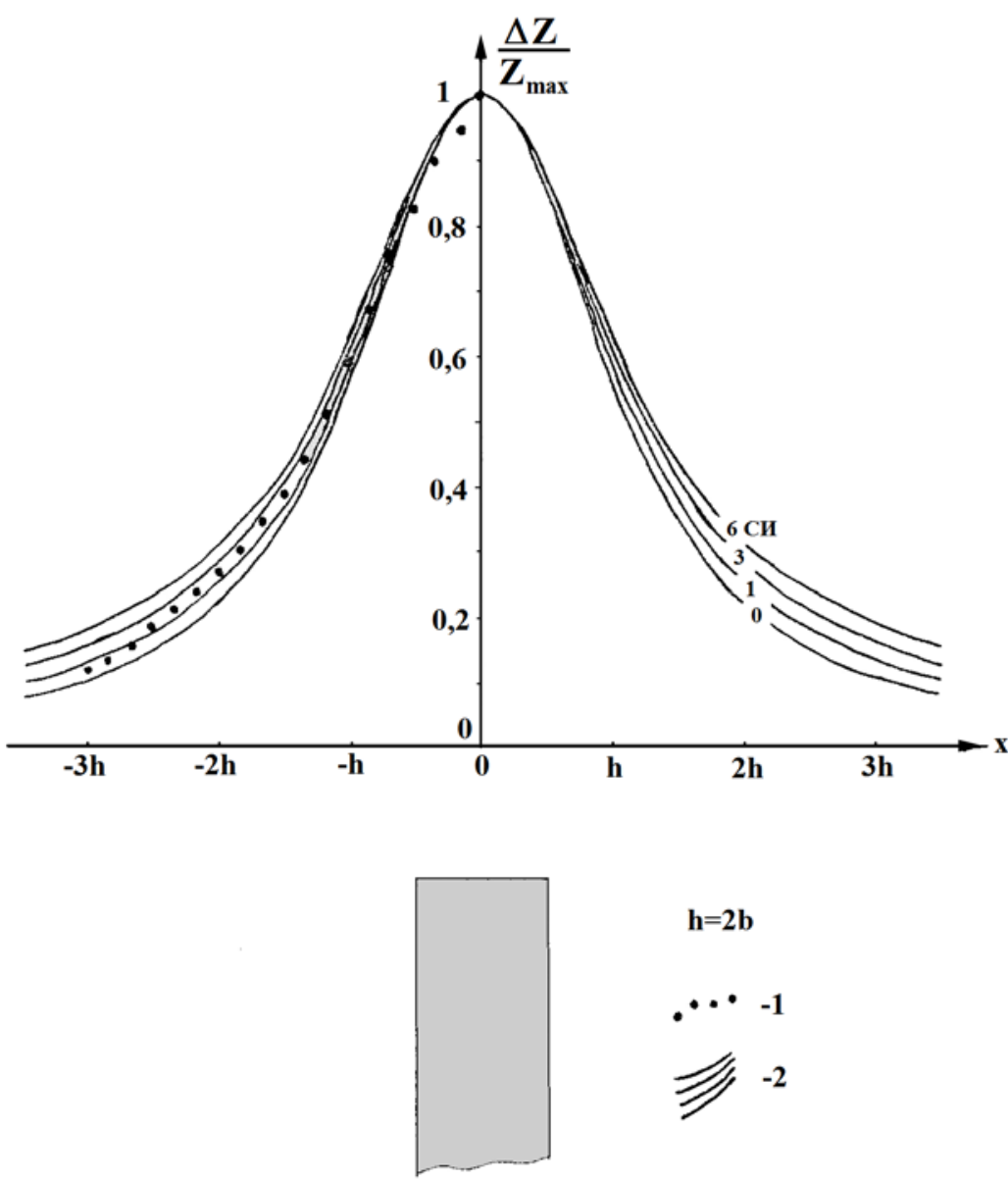

Рис. 49. Определение магнитной восприимчивости по западной ветви графика $\Delta \mathrm{Z}$, изображенного на рис. 48 :

1 - наблюденные значения, нормированные на максимальное; 2 - теоретическое семейство при различных значениях магнитной восприимчивости

руд достаточно хорошо совпали с данными, полученными комплексной магниторазведки с привлечением НЧИМ.

Таким образом, комплексное применение методов интерпретации аномалий земного магнитного поля, имеющих различную чувствительность к проявлениям эффекта 
размагничивания, дает в некоторых случаях возможность определять величину магнитной восприимчивости крутопадающих пластов. Тем не менее, в рамках комплексной магниторазведки подобные определения возможны для гораздо более разнообразных геологических объектов.

\section{§ 18. Основы интерпретации данных магнитовариационного метода}

Как было отмечено во введении, первым из дополняющих традиционную магниторазведку при дистанционном изучении магнитных свойств пород и руд стал магнитовариационный метод (MBM). Решающий вклад в его формирование внес Б.М. Яновский [233]. Суть МВМ заключается в синхронном изучении вариаций геомагнитного поля в базовой точке, находящейся в безаномальной зоне, и в перемещаемой по профилю текущей точке. Выявляя различия в протекании вариаций, по ним получают информацию о магнитной восприимчивости породы.

Развитие метода происходило, можно сказать, драматично. В 1936 г. на одном из участков КМА около села Долгая Поляна Б.М. Яновский провел трехкомпонентные измерения магнитных вариаций, сопоставляя их с аналогичными измерениями в обсерватории Нижнедевицка. Теоретическое обобщение результатов экспериментов было опубликовано в 1938 г., и для интерпретации синхронных трехкомпонентных магнитовариационных измерений Б.М. Яновский достаточно обоснованно для того времени предложил использовать систему уравнений типа Пуассона [233]. Позже, однако, он решил пойти на кардинальное упрощение методики и ограничиться синхронными измерениями только одной - вертикальной компоненты магнитного поля. При этом вопреки своей собственной теории 1938 г. Б.М. Яновский начал совершенно неправомерно утверждать что «вариации вертикальной составляющей над центром аномалии должны быть пропорциональны нормальной вариации» [234, с. 221].

Это заблуждение основоположника сыграло негативную роль во внедрении МВМ, которое тем временем начало разворачиваться и в котором активное участие приняли многие геофизики. Не удивительно, что, обобщив в 1966 г. результаты внедрения, Т.Я. Каменская пришла к выводу о несоответствии теории МВМ с экспериментом [105], что, естественно, затормозило распространение метода.

Серьезную попытку разобраться в причинах выявленного Т.Я. Каменской несоответствия предприняли в 1969 г. В.А. Филатов и В.Н. Филатова, которые справедливо отметили, что большинство применяющих МВМ геофизиков, подобно Б.М. Яновскому, полагают изучаемые объекты намагничивающимися вертикально. Тем самым они «прямо или косвенно предполагают, что влияние горизонтальной компоненты вариаций нормального поля на вариации вертикальной составляющей аномального поля отсутствует» [201, с. 4]. Основное же внимание В.А. Филатов и В.Н. Филатова уделили анализу адекватности теории Б.М. Яновского 1938 г. При этом они показали, что на аномальные вариации сильномагнитных объектов существенное влияние оказывает эффект размагничивания тел в собственном аномальном поле, что ранее в МВМ не учитывалось. Этот эффект приводит к нелинейным зависимостям аномальных вариаций от магнитной восприимчивости изучаемых объектов, что следует учитывать при интерпретации получаемых материалов.

Геофизики позитивно восприняли результаты теоретического анализа и вновь обратились к синхронным трехкомпонентным измерениям вариаций, что позволило достичь определенных успехов. Здесь в первую очередь следует отметить работы Б.А. Ундзенкова [196] и P.В. Тереховой [188], но время в значительной степени было упущено, и на первый план в комплексной магниторазведке тогда выдвинулись МИП и НЧИМ. Тем не менее, исследования возможностей МВМ продолжались, в частности, П.С. Мартышко и В.А. Пьянков доказали единственность двумерной обратной задачи МВМ для эллиптического цилиндра при условии адекватного учета его размагничивания [132]. Однако до настоящего времени теоретические основы МВМ остаются недостаточно проработанными, особенно применительно к анизотропным объектам, таким как пласты железистых кварцитов, что требует устранения. 
Рассмотрим вкратце традиционные основы МВМ. Как известно [39], во внешней точке аномальная индукция $\overrightarrow{\mathrm{B}}(\mathrm{a})$ произвольного трехмерного объекта V может быть представлена в следующем матричном виде:

$$
\overrightarrow{\mathrm{B}}(\mathrm{a})=\frac{\mu_{0}}{4 \pi} \int_{\mathrm{V}} \nabla^{2}\left(\frac{1}{\mathrm{~L}_{\mathrm{qa}}}\right) \overrightarrow{\mathrm{I}}(\mathrm{q}) \mathrm{dV},
$$

где $\overrightarrow{\mathrm{I}}(\mathrm{q})$ - намагниченность объекта во внутренней точке $\mathrm{q}, \mathrm{L}_{\mathrm{qa}}$ - расстояние между точками q и а - той, где вычисляется поле; $\nabla^{2}\left(\frac{1}{\mathrm{~L}_{\mathrm{qa}}}\right)$ - матрица вторых производных (матрица Гессе) функции $\frac{1}{\mathrm{~L}_{\mathrm{qa}}}$. Если придерживаться, подобно Б.М. Яновскому, традиционной упрощенной концепции, то намагниченность сложного объекта можно приближенно полагать однородной и вычислять ее с помощью коэффициентов размагничивания эквивалентного эллипсоида. Эллипсоид при этом удобен тем, что в однородном намагничивающем поле он намагничивается однородно, тогда как тела неэллипсоидальных форм под влиянием размагничивания даже в однородном намагничивающем поле приобретают неоднородную намагниченность, распределение которой зависит от формы тела. Для однородно намагниченного эллипсоида формулу (18.1), очевидно, можно переписать в более простом виде:

$$
\overrightarrow{\mathrm{B}}(\mathrm{a})=\left[\frac{\mu_{0}}{4 \pi} \int_{\mathrm{V}} \nabla^{2}\left(\frac{1}{\mathrm{~L}_{\mathrm{qa}}}\right) \mathrm{dV}\right] \overrightarrow{\mathrm{I}} .
$$

Раскрыв (18.2), Б.М. Яновский представил основную систему уравнений типа Пуассона, описывающую аномальные магнитовариационные поля, в следующей форме:

$$
\begin{gathered}
\delta \mathrm{X}-\delta \mathrm{X}_{0}=\kappa_{\mathrm{x}}^{*} \mathrm{c}_{\mathrm{xx}} \delta \mathrm{X}_{0}+\kappa_{\mathrm{y}}^{*} \mathrm{c}_{\mathrm{xy}} \delta \mathrm{Y}_{0}+\kappa_{\mathrm{z}}^{*} \mathrm{c}_{\mathrm{xz}} \delta \mathrm{Z}_{0}, \\
\delta \mathrm{Y}-\delta \mathrm{Y}_{0}=\kappa_{\mathrm{x}}^{*} \mathrm{c}_{\mathrm{yx}} \delta \mathrm{X}_{0}+\kappa_{\mathrm{y}}^{*} \mathrm{c}_{\mathrm{yy}} \delta \mathrm{Y}_{0}+\kappa_{\mathrm{z}}^{*} \mathrm{c}_{\mathrm{yz}} \delta \mathrm{Z}_{0}, \\
\delta \mathrm{Z}-\delta \mathrm{Z}_{0}=\kappa_{\mathrm{x}}^{*} \mathrm{c}_{\mathrm{zx}} \delta \mathrm{X}_{0}+\kappa_{\mathrm{y}}^{*} \mathrm{c}_{\mathrm{zy}} \delta \mathrm{Y}_{0}+\kappa_{\mathrm{z}}^{*} \mathrm{c}_{\mathrm{zz}} \delta \mathrm{Z}_{0} .
\end{gathered}
$$

Здесь $\delta \mathrm{X}_{0}, \delta \mathrm{Y}_{0}$ и $\delta \mathrm{Z}_{0}$ - компоненты вариации индукции нормального геомагнитного поля $\delta \overrightarrow{\mathrm{B}}_{0}$, измеренные в базовой точке, а $\delta \mathrm{X}, \delta \mathrm{Y}$ и $\delta \mathrm{Z}$, соответственно компоненты вариации индукции в изучаемом пункте. Индукционные параметры, обозначаемые как с, зависят от формы и расположения эллипсоида, образуют симметричную матрицу и по сравнению с теми, которые ввел Б.М. Яновский, из-за использования системы единиц СИ должны быть уменьшены в $\mu_{0}$ раз. Их физический смысл достаточно прост, например, $\mathrm{c}_{\mathrm{zx}}$ представляет собой компоненту Z аномального поля эллипсоида в пункте измерений, рассчитанную при единичной намагниченности, ориентированной вдоль оси Ох, и нормированную затем на $\mu_{0}$. Параметр $\mathrm{c}_{\mathrm{xy}}$ является компонентой $\mathrm{X}$ аномального поля эллипсоида, рассчитанной при единичной намагниченности, направленной вдоль оси Оу и нормированной на $\mu_{0}$, и т.д. Величины $\kappa_{\mathrm{x}}^{*}, \kappa_{\mathrm{y}}^{*}$ и $\kappa_{\mathrm{z}}^{*}$ в (18.3) - так называемые кажущиеся магнитные восприимчивости по соответствующим осям.

Стоит отметить, что Б.А. Ундзенков именует (18.3) системой уравнений ПуассонаАрчибальда Смита [196]. Действительно, этот английский адвокат и математик (Archibald Smith, 1813-1872) применял уравнения Пуассона для изучения девиации морских корабельных компасов, добавив в них постоянные слагаемые, описывающие влияние остаточной намагниченности судов, но как раз в МВМ эти добавки не нужны. Сам же А. Смит, кстати, даже модифицированную им систему называл системой уравнений Пуассона, поэтому мы будем далее использовать именно это название.

Компоненты вектора намагниченности $\mathrm{I}_{\mathrm{x}}, \mathrm{I}_{\mathrm{y}}$ и $\mathrm{I}_{\mathrm{z}}$ эллипсоидов вычисляются по простым формулам. Так, в случае, когда оси однородного и изотропного эллипсоида с магнитной восприимчивостью к параллельны координатным осям, соответственно:

$$
\mathrm{I}_{\mathrm{x}}=\frac{\kappa \mathrm{X}_{0}}{\mu_{0}\left(1+\kappa \mathrm{N}_{\mathrm{x}}\right)}, \mathrm{I}_{\mathrm{y}}=\frac{\kappa \mathrm{Y}_{0}}{\mu_{0}\left(1+\kappa \mathrm{N}_{\mathrm{y}}\right)}, \mathrm{I}_{\mathrm{x}}=\frac{\kappa \mathrm{Z}_{0}}{\mu_{0}\left(1+\kappa \mathrm{N}_{\mathrm{z}}\right)},
$$

где $\mathrm{N}_{\mathrm{x}}, \mathrm{N}_{\mathrm{y}}$ и $\mathrm{N}_{\mathrm{z}}$ - коэффициенты размагничивания эллипсоида по этим осям [39]. Вводя кажущиеся магнитные восприимчивости стандартными соотношениями 
Ю.И. Блох

$$
\kappa_{\mathrm{x}}^{*}=\frac{\kappa}{1+\kappa N_{\mathrm{x}}}, \kappa_{\mathrm{y}}^{*}=\frac{\kappa}{1+\kappa N_{\mathrm{y}}}, \kappa_{\mathrm{z}}^{*}=\frac{\kappa}{1+\kappa \mathrm{N}_{\mathrm{z}}},
$$

(18.4) можно преобразовать к виду

$$
\mathrm{I}_{\mathrm{x}}=\frac{\kappa_{\mathrm{x}}^{*} \mathrm{X}_{0}}{\mu_{0}}, \mathrm{I}_{\mathrm{y}}=\frac{\kappa_{\mathrm{y}}^{*} \mathrm{Y}_{0}}{\mu_{0}}, \mathrm{I}_{\mathrm{x}}=\frac{\kappa_{\mathrm{z}}^{*} \mathrm{Z}_{0}}{\mu_{0}} .
$$

Если рассматриваемый эллипсоид анизотропен, оси его параллельны координатным осям, а магнитные восприимчивости в направлении этих осей равны $\kappa_{\mathrm{x}}$, $\kappa_{\mathrm{y}}$ и $\kappa_{\mathrm{z}}$, компоненты его намагниченности тоже вычисляются по формуле (18.6), но кажущиеся магнитные восприимчивости, естественно, определяются несколько иначе:

$$
\kappa_{\mathrm{x}}^{*}=\frac{\kappa_{\mathrm{x}}}{1+\kappa_{\mathrm{x}} \mathrm{N}_{\mathrm{x}}}, \kappa_{\mathrm{y}}^{*}=\frac{\kappa_{\mathrm{y}}}{1+\kappa_{\mathrm{y}} \mathrm{N}_{\mathrm{y}}}, \kappa_{\mathrm{z}}^{*}=\frac{\kappa_{\mathrm{z}}}{1+\kappa_{\mathrm{z}} \mathrm{N}_{\mathrm{z}}} .
$$

Отметим, что наиболее практически важным является частный случай эллипсоида с полосчатой структурой, у которого магнитная восприимчивость в направлении нормали к полосчатости (поперечная) равна $\kappa_{\mathrm{n}}$, а в направлении двух других (вдоль полосчатости) осей магнитные восприимчивости (продольные) одинаковы и равны $\kappa_{\mathrm{t}}$, причем $\kappa_{\mathrm{t}}>\kappa_{\mathrm{n}}$.

Приведенные формулы позволяют достаточно просто оценивать компоненты тензора магнитной восприимчивости эллипсоидальных тел, но для этого, очевидно, форма изучаемого объекта должна быть заранее известна. В общем же случае обратная задача не имеет единственного решения. Если же форма и расположение изучаемого источника известны, то применяемую при оценке параметров по формулам (18.3) декартову систему координат наиболее удобно заранее развернуть так, чтобы ее оси были ориентированы вдоль соответствующих осей эллипсоида. После этого расчеты коэффициентов при $\delta \mathrm{X}_{0}, \delta \mathrm{Y}_{0}$ и $\delta \mathrm{Z}_{0}$ в (18.3) по аномальным вариациям окажутся максимально простыми и сведутся к решению линейных обратных задач [Приложение].

Для объектов неэллипсоидальных форм интерпретация более сложна, но ее можно выполнять путем численного моделирования на основе приведенного в главе 2 алгоритма решения прямых задач для трехмерных анизотропных геологических объектов с учетом размагничивания.

Рассмотрим сравнительно простой, но методически важный пример подобного моделирования, адекватно учитывающего

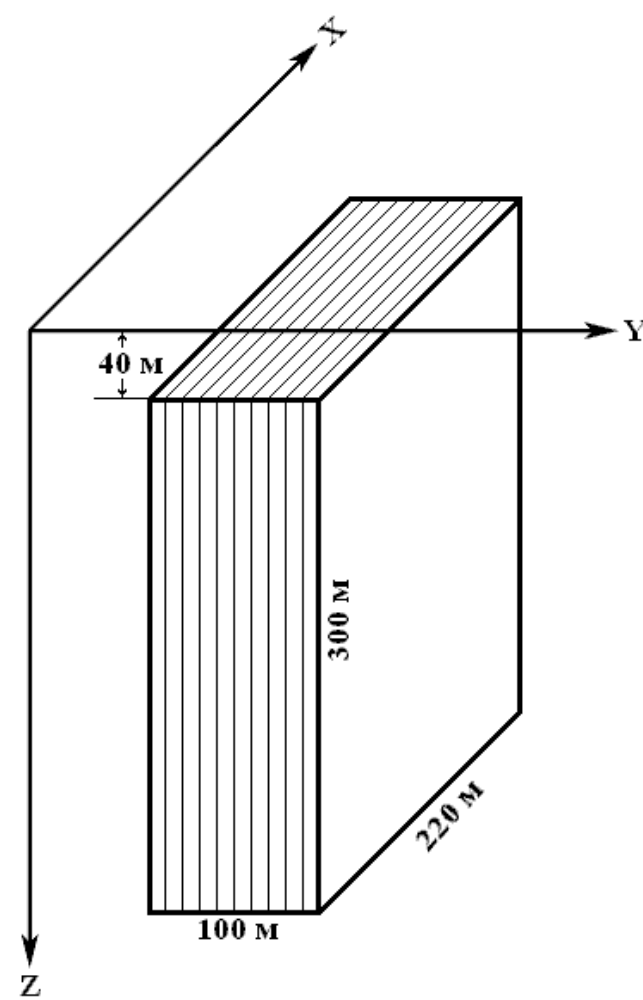

Рис. 50. Моделируемая анизотропная призма как эффект размагничивания, так и анизотропию объекта. Изучаемым объектом будем считать вертикальную призму, расположенную как показано на рис. 50, а в качестве изменяющегося во времени нормального магнитного поля воспользуемся реальным полем, наблюдавшимся в течение суток геомагнитной обсерваторией Москва (ИЗМИРАН). Обратим внимание на то, что принятая частная ориентация призмы не ограничивает общности примера, поскольку, как было отмечено выше, при оценке анизотропии известных тел оси координат можно поворачивать наиболее удобным образом. Нормаль к полосчатости модели, как видно из рис. 50, ориентирована вдоль оси Оу (на восток), и магнитная восприимчивость вдоль нее $\left(\kappa_{\mathrm{y}}=\kappa_{\mathrm{n}}\right)$ принята равной 1 СИ, а вдоль осей Ox и Oz считается одинаковой $\left(\kappa_{\mathrm{x}}=\kappa_{\mathrm{z}}=\kappa_{\mathrm{t}}\right)$ и равной 1,5 СИ. Аномальное магнитное поле $\Delta \mathrm{T}$ данной модели, остаточная намагниченность которой 
считалась отсутствующей, рассчитано для региона Москвы и представлено на рис 51, а. Как видно, амплитуда вычисленной аномалии превышает 10 мкТл. Отметим, что в соответствии с известным критерием подобия магнитных полей [39], вообще говоря, все линейные размеры в задаче можно пропорционально изменять, но, если магнитные параметры при этом не изменяются, то не изменятся и аномальные поля модели.

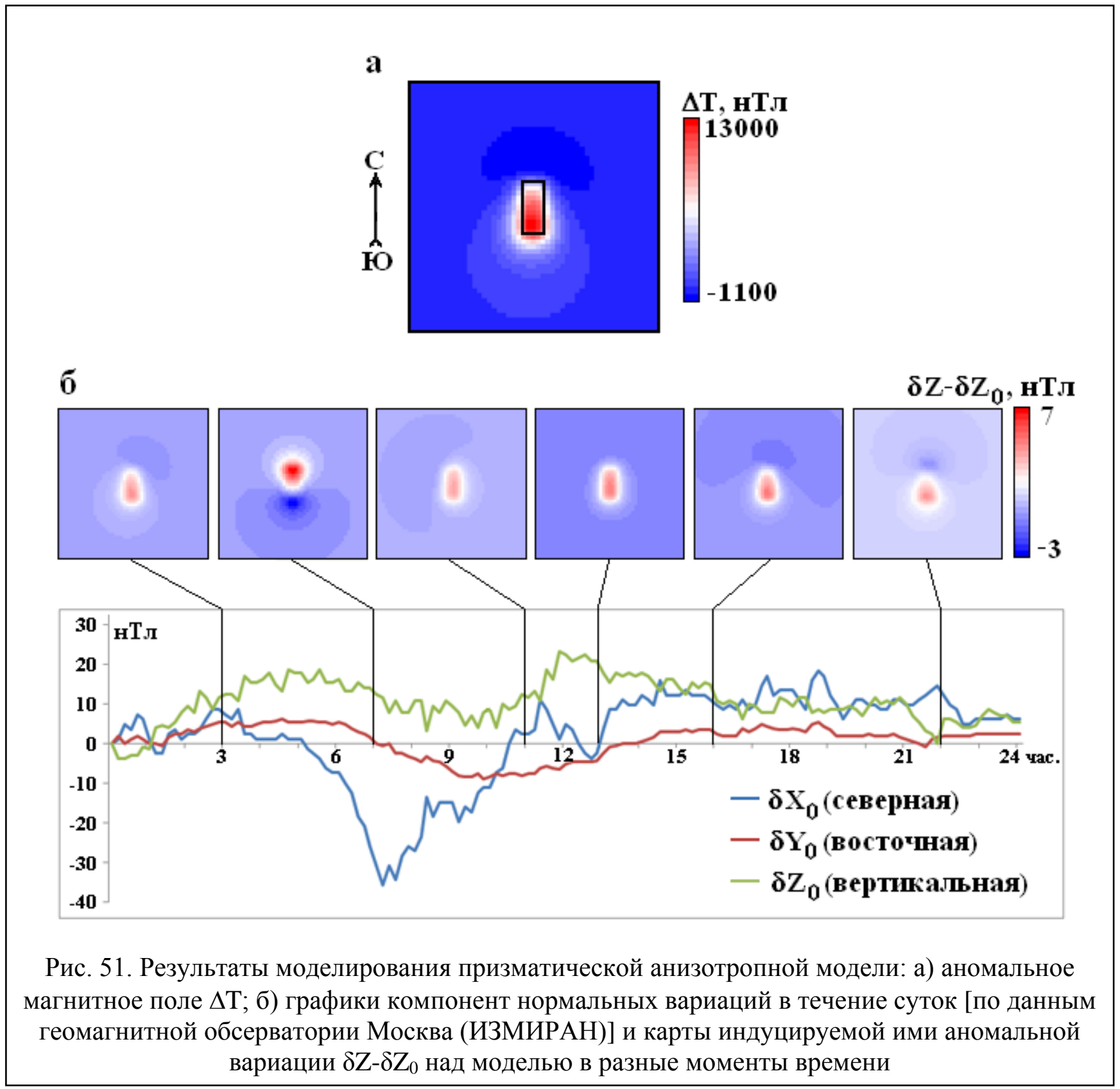

Чтобы рассчитать аномальные магнитовариационные поля в произвольный момент времени, интегральное уравнение для намагниченности модели достаточно численно решить всего трижды для единичных компонент вариаций индукции нормального геомагнитного поля, то есть $\delta \mathrm{X}_{0}=1, \delta \mathrm{Y}_{0}=1$ и $\delta \mathrm{Z}_{0}=1$. После этого в силу принципа суперпозиции полей любые компоненты аномальной вариации в любой из точек для любого момента времени вычисляются просто как суммы соответствующих произведений.

На рис. 51, б представлены карты аномальной вариации $\delta \mathrm{Z}-\delta \mathrm{Z}_{0}$ над данной моделью для шести моментов времени в течение суток. Они четко демонстрируют, что в разные времена вариации нормального поля приводят к различным амплитудам и морфологиям аномальных полей. Иногда нормальная вариация субвертикальна, но в течение гораздо большего времени 
она субгоризонтальна. Формальная линейная аппроксимация связи между $\delta Z$ и $\delta Z_{0}$ приводит в данном случае к уравнению $\delta Z=1,119 \cdot \delta Z_{0}$, но среднеквадратическая погрешность такой аппроксимации оказывается равной 1,6 нТл, а максимальные отклонения достигают 6-7 нТл, то есть сопоставимы с самими аномальными вариациями. Никакой пропорциональности между $\delta Z$ и $\delta Z_{0}$, предполагавшейся Б.М. Яновским, здесь, очевидно, нет. Пример, скорее, подтверждает мнение В.А. Филатова и В.Н. Филатовой о том, что «по нарушению прямой пропорциональности между вариациями $\delta Z$ и $\delta Z_{0}$ можно судить о большой величине магнитной восприимчивости вещества» [201, с. 17]. Хотя такое суждение носит качественный характер, оно может быть практически полезным.

На рис. 52 карты аномальной вариации $\delta Z-\delta Z_{0}$ над той же моделью показаны для времени магнитной бури, К-индекс которой достигает 7. Они демонстрируют субгоризонтальность вектора нормальных вариаций и во время сильных возмущений магнитосферы.

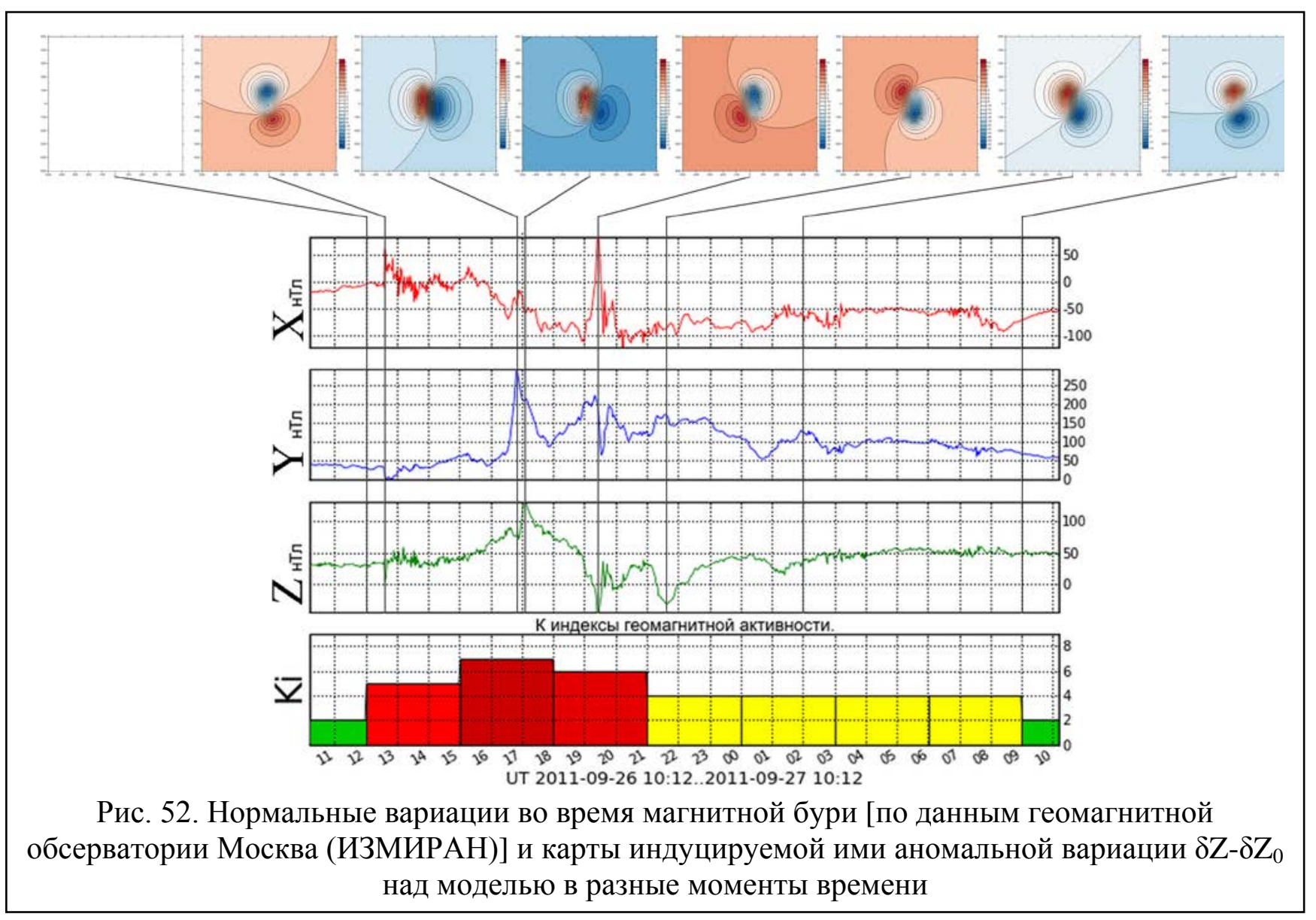

Важнейшим в МВМ является вопрос о влиянии электропроводности горных пород и руд. Известно, что характер магнитных вариаций изменяется под влиянием не только магнитных свойств геологических объектов, но и их удельного электрического сопротивления, на чем базируются разнообразные магнитотеллурические методы [153]. В начале 70-х годов М.Л. Финкельштейн провел оценку влияния проводящих тел на результаты МВМ [206], опираясь на результаты Дж. Уэйта и С. Уорда, в частности, на аналоги приведенных на рис. 8 графиков частотных характеристик шара. В результате им был сделан совершенно справедливый вывод о необходимости использовать для МВМ особо низкочастотные, желательно, суточные магнитные вариации. Именно они позволяют работать в области левой (низкочастотной) асимптоты частотной характеристики и интерпретировать результаты по формулам магнитостатики.

Со времен первых работ Б.М. Яновского для изучения магнитных вариаций в МВМ использовались те пункты в пределах аномалии, где она достигает своих экстремальных 
значений при съемке традиционной магниторазведкой [233]. Если изучаемый объект изотропен, такой прием вполне допустим, но для анизотропных тел он становится крайне неудачным. Например, если в анализируемом примере выбрать для съемки точку максимума $\Delta \mathrm{T}$, которая расположена практически над меридиональной плоскостью симметрии модели, то в ней анизотропия вообще никак не будет сказываться, поскольку в силу симметрии модели нормальная вариация $\delta Y_{0}$ здесь, очевидно, вообще не влияет на аномалию $\delta Z$. То же относится к любым точкам на профиле, лежащем в этой плоскости симметрии модели. В точках же, расположенных в другой плоскости симметрии модели, параллельной осям координат Oy и Oz, влияния на аномалию $\delta Z$ не будет оказывать вариация $\delta \mathrm{X}_{0}$ и т.д. В рассматриваемом примере наиболее удобными для изучения анизотропии магнитной восприимчивости, видимо, можно считать точки вблизи проекций вершин призмы на дневную поверхность. В них и аномалии достаточно велики, и от плоскостей симметрии они удалены. Подобный анализ легко проводить и для объектов других форм.

Еще один методический вопрос связан с выбором изучаемых компонент поля вариаций. Очевидно, наилучшим вариантом проведения работ является синхронное измерение всех трех компонент поля. Тем не менее, можно применять и более простые варианты, когда в нормальном поле на базовой точке измеряются все три компоненты $\delta \mathrm{X}_{0}, \delta \mathrm{Y}_{0}$ и $\delta \mathrm{Z}_{0}$, а над объектом - лишь одна, например, $\delta Z$ или $\delta \mathrm{T}$. Главное - это непременное измерение всех трех компонент на базовой точке, чего в общем случае не способны заменить никакие паллиативные приемы.

Приведенные формулы для эллипсоидов, естественно не вполне пригодны для более сложных по форме геологических тел и, чтобы оценить их точность, продолжим рассмотрение примера с анизотропной призмой.

В наиболее общем матричном виде систему уравнений Пуассона (18.3), описывающих линейную связь вектора аномальных магнитных вариаций $\delta \overrightarrow{\mathrm{B}}_{a}=\delta \overrightarrow{\mathrm{B}}-\delta \overrightarrow{\mathrm{B}}_{0}$ с вектором нормальных вариаций $\delta \overrightarrow{\mathrm{B}}_{0}$, можно представить следующим образом:

$$
\delta \overrightarrow{\mathrm{B}}_{a}=\Phi \cdot \delta \overrightarrow{\mathrm{B}}_{0}
$$

Матрица связи здесь вслед за Б.А. Ундзенковым обозначена как Ф. Если пренебречь реальным влиянием размагничивания, ее можно представить в виде произведения $\Phi=\mathrm{C} \kappa^{*}$, где $\kappa^{*}-$ тензор кажущейся магнитной восприимчивости, а С - состоящая из 9 элементов матрица индукционных параметров. Поскольку при определении анизотропии магнитной восприимчивости объектов, их местоположение и форму надо знать заранее, матрицу С можно рассчитать, например, для варианта изотропной магнитной восприимчивости, равной 1 СИ, с учетом размагничивания. Элементы матрицы $\Phi$ в свою очередь независимо и однозначно определяются по данным МВМ путем решения соответствующих линейных обратных задач [Приложение]. После этого тензор к* может быть оценен по формуле

$$
\kappa^{*}=\mathrm{C}^{-1} \Phi,
$$

где $\mathrm{C}^{-1}$ - матрица, обратная к С. Если бы объект являлся слабомагнитным пластом, а оси координат были ориентированы с учетом симметрии модели, тензор к* являлся бы диагональной матрицей с искомыми значениями $\kappa_{\mathrm{t}}$ и $\kappa_{\mathrm{n}}$. На самом деле из-за влияния эффекта размагничивания вид получаемой матрицы более сложен. Для рассматриваемого примера, в частности, матрица, полученная таким образом по данным измерений в единственной точке, выбранной исходя из изложенных соображений, имела бы следующий вид:

$$
\kappa^{*}=\left(\begin{array}{ccc}
1,316 & 0,036 & 0,065 \\
0,107 & 1,143 & -0,263 \\
0,034 & -0,113 & 1,304
\end{array}\right) \text {. }
$$

Тем не менее, полученные столь просто оценки компонент тензора кажущейся магнитной восприимчивости, а именно $\kappa_{\mathrm{t}}=1,3$ СИ и $\kappa_{\mathrm{n}}=1,1$ СИ являются вполне приемлемыми начальными 
приближениями для последующего детального моделирования с адекватным учетом размагничивания. Без такого моделирования не только для анизотропных, но и для заведомо изотропных объектов в настоящее время интерпретация данных МВM вообще не представляется возможной.

Таким образом, с одной стороны, численное моделирование показывает весьма существенную зависимость результатов МВМ от анизотропии магнитной восприимчивости изучаемых геологических объектов. С другой стороны, оно демонстрирует возможности ее адекватной и нетрудоемкой оценки с учетом эффекта размагничивания объекта в собственном аномальном поле, что позволяет разделить влияния на поля анизотропии магнитной восприимчивости и анизотропии формы объекта. Тем самым современные технологии моделирования предоставляют возможности эффективного применения магнитовариационного метода при изучении анизотропных геологических объектов в их естественном залегании.

\section{§ 19. Основы интерпретации данных многочастотных измерений в комплексной магниторазведке}

Анализ основ метода искусственного подмагничивания (МИП) и низкочастотных индуктивных методов (НЧИМ) в главе 1 показал их существенную теоретическую близость. Фактически первоначальные варианты МИП являлись частными случаями применения НЧИМ при нулевой частоте первичного поля, а специфика МИП сводилась к применению компенсирующих многовитковых петель. В поздних же вариантах МИП все шире стали применяться индукционные преобразователи, измеряющие аномальные эффекты в гармонически меняющихся полях частотой от десятых долей до нескольких Гц [170], и тем самым практические аспекты применения НЧИМ и МИП также неуклонно сближались.

Чрезвычайно важно понимать, что, вообще говоря, возможности многочастотных измерений принципиально превышают возможности любых одночастотных модификаций, и это легко показать на простом примере проводящего и магнитного шара. Дело в том, что по данным многочастотных измерений обратная задача для такого шара решается однозначно, тогда как по данным измерений на одной частоте она, как и в традиционной магниторазведке [39], не имеет единственного решения.

Единственность решения обратной задачи для проводящего и магнитного шара по данным многочастотных измерений доказал в 1959 г. С. Уорд [260], и методическая важность этого результата побуждает к его подробному рассмотрению. При этом во избежание недоразумений, необходимо сразу же оговорить то, что оригинальная статья С. Уорда содержит несколько иные обозначения по сравнению с применяемыми в отечественной литературе и введенными в главе 1. Так, вместо параметра $\mathrm{p}^{2}=\gamma \mu \mathrm{a}^{2} / 2$, в зависимости от которого построены графики действительной и мнимой составляющей комплексной функции $\mathrm{D}(\mu)$ на рис. 8 , С. Уорд использует параметр $\theta=\mathrm{a} \sqrt{\gamma \mu \omega}=\mathrm{p} \sqrt{2}$, а вместо $\mathrm{D}(\mu)-$ функцию $\mathrm{M}+\mathrm{iN}=\mathrm{D} / 3$. Далее мы будем пользоваться обозначениями, привычными для отечественных геофизиков, и лишь кратко комментировать вид соотношений, которыми оперировал С. Уорд. Еще одно отличие от того, что излагалось в статье С. Уорда, связано с преимущественным рассмотрением измеряемой обычно на практике вертикальной компоненты аномального поля, тогда как в статье анализируется его горизонтальная компонента.

Применительно к задачам комплексной магниторазведки главное - это определение магнитной восприимчивости $\kappa$ шара либо связанной с ней соотношением $\mu=1+\kappa$ относительной магнитной проницаемости. Эти параметры шара можно определить по данным многочастотных измерений в одной единственной точке. Единственное требование к ее расположению связано с тем, чтобы аномалия измеряемой компоненты поля (чаще вертикальной) в ней не была нулевой на всех частотах. Подчеркнем, что для определения относительной магнитной проницаемости шара не требуются априорные знания ни о его действительном местоположении, ни о радиусе, ни о проводимости. 
Полученные результаты многочастотных измерений позволяют вычислить в интересующей точке набор аномальных значений. Как отмечалось в $§ 2$, в НЧИМ аномалии вертикальной составляющей индукции $\mathrm{B}_{\mathrm{z}}^{\mathrm{a}}$ вычисляются обычно по измеренным значениям $\mathrm{B}_{\mathrm{z}} \mathrm{B}$ долях нормального поля в данной точке $\mathrm{B}_{\mathrm{z}}^{0}$ :

$$
\mathrm{B}_{\mathrm{z}}^{\mathrm{a}}=\frac{\mathrm{B}_{\mathrm{z}}-\mathrm{B}_{\mathrm{z}}^{0}}{\mathrm{~B}_{\mathrm{z}}^{0}}
$$

и выражаются в \%. Построив график $\mathrm{B}_{\mathrm{z}}^{\mathrm{a}}(\omega)$ (частотную характеристику), интерпретатор оценивает значения на асимптотах - при этом на низкочастотной асимптоте $\mathrm{B}_{\mathrm{z}}^{\mathrm{a}}(0)>0$, a на высокочастотной $\mathrm{B}_{\mathrm{z}}^{\mathrm{a}}(\infty)<0$. По отношению асимптотических значений и определяется отношение $\mu / \mu_{\mathrm{e}}$, для чего используется формула (3.40), из которой следует, что

$$
\frac{\mathrm{B}_{\mathrm{z}}^{\mathrm{a}}(0)}{\mathrm{B}_{\mathrm{z}}^{\mathrm{a}}(\infty)}=-2\left[\frac{\mu / \mu_{e}-1}{\mu / \mu_{\mathrm{e}}+2}\right] \text {. }
$$

На практике чаще всего внешняя среда практически немагнитна, то есть $\mu_{\mathrm{e}}=1$, и тогда

$$
\mu=2 \cdot \frac{1-\mathrm{B}_{\mathrm{z}}^{\mathrm{a}}(0) / \mathrm{B}_{\mathrm{z}}^{\mathrm{a}}(\infty)}{2+\mathrm{B}_{\mathrm{z}}^{\mathrm{a}}(0) / \mathrm{B}_{\mathrm{z}}^{\mathrm{a}}(\infty)} .
$$

Еще раз обратим внимание на то, что магнитная проницаемость шара однозначно определяется только благодаря многочастотным измерениям и возможностям анализа частотной характеристики. Если бы шар был абсолютно непроводящим, подобные определения стали бы невозможны, следовательно, и обратная задача перестала бы решаться единственно.

Следующим шагом интерпретации является определение местоположения центра шара, что можно осуществлять любым из методов, разработанных в традиционной магниторазведке. Удобно выполнять это методами особых точек, хотя можно ограничиться и простейшими разновидностями подбора по характерным точкам графиков аномальных полей, что предлагал С. Уорд [260]. Горизонтальные координаты центра шара совпадают с координатами максимума $\mathrm{B}_{\mathrm{z}}^{\mathrm{a}}$, а для определения его глубины на профиле,

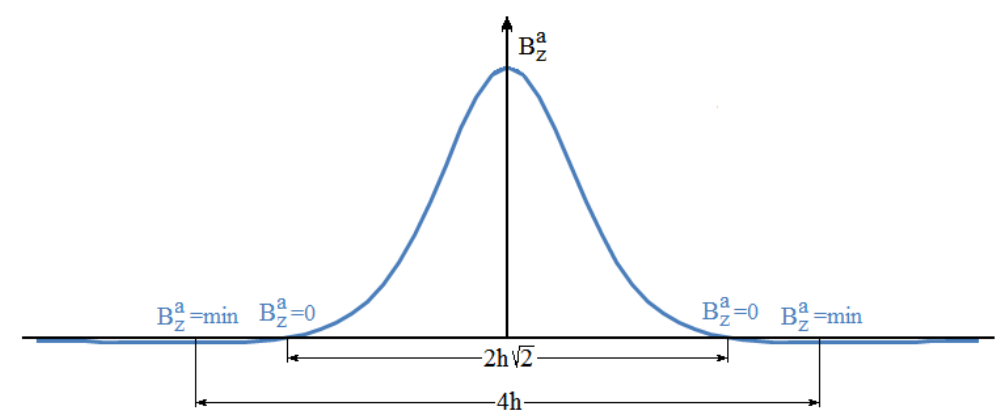

Рис. 53. Определение глубины центра шара $\mathrm{h}$ по характерным точкам графика $\mathrm{B}_{\mathrm{z}}^{\mathrm{a}}$ проходящем через эпицентр, можно воспользоваться характерными точками графика $\mathrm{B}_{\mathrm{z}}^{\mathrm{a}}$, показанными на рис. 53. Расстояние между точками минимума $\mathrm{B}_{\mathrm{z}}^{\mathrm{a}}$, как видно, равно 4h. Эти точки не очень четко идентифицируются, поэтому лучше использовать точки перехода графика $\mathrm{B}_{\mathrm{z}}^{\mathrm{a}}$ через ось абсцисс, расстояние между которыми равно $2 \mathrm{~h} \sqrt{2}$. С. Уорд в своей статье рекомендовал использовать точки экстремумов нечетно симметричного графика горизонтальной компоненты аномального поля, расстояние между которым равно $\mathrm{h}$, но эта компонента в практике комплексной магниторазведки измеряется крайне редко.

Далее определяется радиус шара, для чего можно использовать максимальное значение $\mathrm{B}_{\mathrm{z}}^{\mathrm{a}}$ в эпицентре, естественно, не в процентах, а просто в долях нормального поля. Так как магнитная проницаемость шара $\mu$ уже найдена с помощью частотной характеристики, из формул (3.24) и (3.44) следует, что

$$
\mathrm{a}^{3}=\mathrm{B}_{\mathrm{z} \max }^{\mathrm{a}} \frac{\mu+2}{\mu-1} \frac{\mathrm{h}^{3}}{2} .
$$

Остается определить проводимость шара $\gamma$, для чего можно воспользоваться значением параметра $\mathrm{p}^{2}=\gamma \mu \omega \mathrm{a}^{2} / 2$ в одной из характерных точек частотной характеристики. С. Уорд 
предложил использовать для этого точку, где частотная характеристика переходит через 0 , так

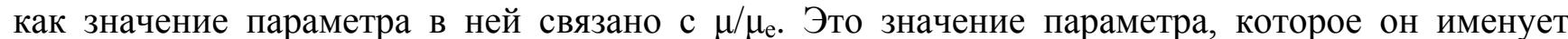
критическим и обозначает $\theta_{c}$, предлагалось определять по эмпирической формуле $\theta_{c}=1,84 \mu / \mu_{\mathrm{e}}$ либо же по приводимому в его статье графику. Как отмечалось, применяемый им параметр $\theta$ связан с параметром р соотношением $\theta=\mathrm{p} \sqrt{2}$, поэтому

откуда следует

$$
\mathrm{p}_{\mathrm{c}}^{2}=\frac{\theta_{\mathrm{c}}^{2}}{2}=1,69\left(\frac{\mu}{\mu_{\mathrm{e}}}\right)^{2}
$$

$$
\gamma=\frac{3,38}{\mu \omega \mathrm{a}^{2}}\left(\frac{\mu}{\mu_{\mathrm{e}}}\right)^{2}
$$

Таким образом, все параметры шара определяются однозначно. В комплексной магниторазведке геофизики проводят и традиционную магнитную съемку, результаты которой помимо магнитной проницаемости шара зависят также от его естественной остаточной намагниченности. Зная уже найденные параметры, а также компоненты нормального геомагнитного поля в районе наблюдений, компоненты вектора естественной остаточной намагниченности легко найти путем решения соответствующей линейной обратной задачи [Приложение]. Если же в геофизический комплекс входит и гравиразведка, то, зная радиус шара по данным комплексной магниторазведки, по гравитационной аномалии элементарно найти и его избыточную плотность.

\section{§ 20. Возможности многопетлевых измерений в комплексной магниторазведке}

Продолжим рассмотрение возможностей, недоступных для традиционной магниторазведки, но неотъемлемо присущих комплексной магниторазведке. Среди них большое значение имеют те, которые базируются на управлении полями искусственных источников в МИП и НЧИМ: их величиной и направлением в разных частях пространства.

Если рассмотреть многопетлевые исследования с точки зрения единственности решения соответствующих обратных задач, то здесь в первую очередь следует обратить внимание на исследования М.М. Лаврентьева. Из них следует, что, проведя измерения аномального поля при непрерывно (то есть практически многократно) перемещаемом источнике подмагничивающего поля, можно получить однозначное решение обратной задачи [119, 170, 216]. Дальнейшие

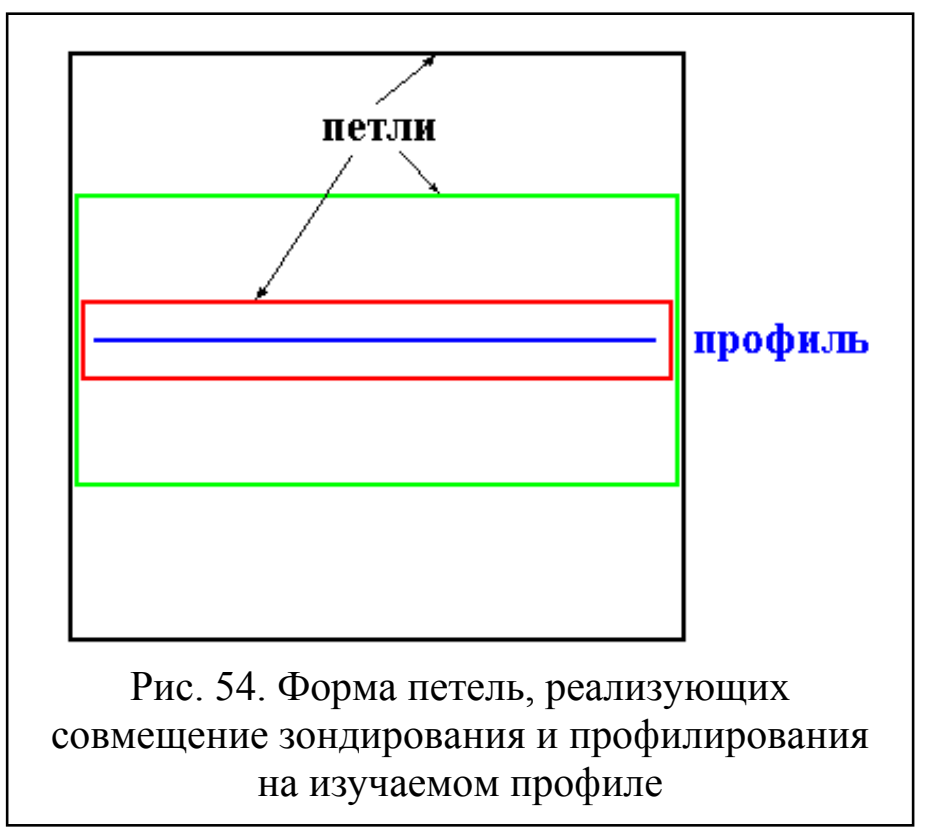
исследования, выполненные В.А. Филатовым, В.Н. Страховым, А.В. Цирульским и П.С. Мартышко, позволили уменьшить жесткость накладываемых ограничений и доказали, что в довольно общей ситуации для достижения единственности получаемого решения достаточно провести измерения всего в двух разных источниках подмагничивающего поля $[129,178,216]$. 
Ю.И. Блох

Теоретические основы комплексной магниторазведки

Наиболее популярными среди тех применяющихся на практике методик, которые основаны на управлении первичным полем, являются индукционные зондирования. Такие зондирования с круглыми петлями изменяющихся радиусов для изучения проводящих разрезов еще в 30-х годах прошлого века предлагал применять И. Кёнигсбергер (Johann Georg Königsberger, 1874-1946). Применительно к изучению магнитных объектов методики зондирования НЧИМ, использующие квадратные и прямоугольные петли, активно разрабатывал И.В. Ренард [45, 151, 232]. В МИП широко применялось магнитное зондирование с соосными квадратными компенсирующими рамками размерами от 200 м до 2 км, разработанное В.Д. Стадухиным и др. [170].

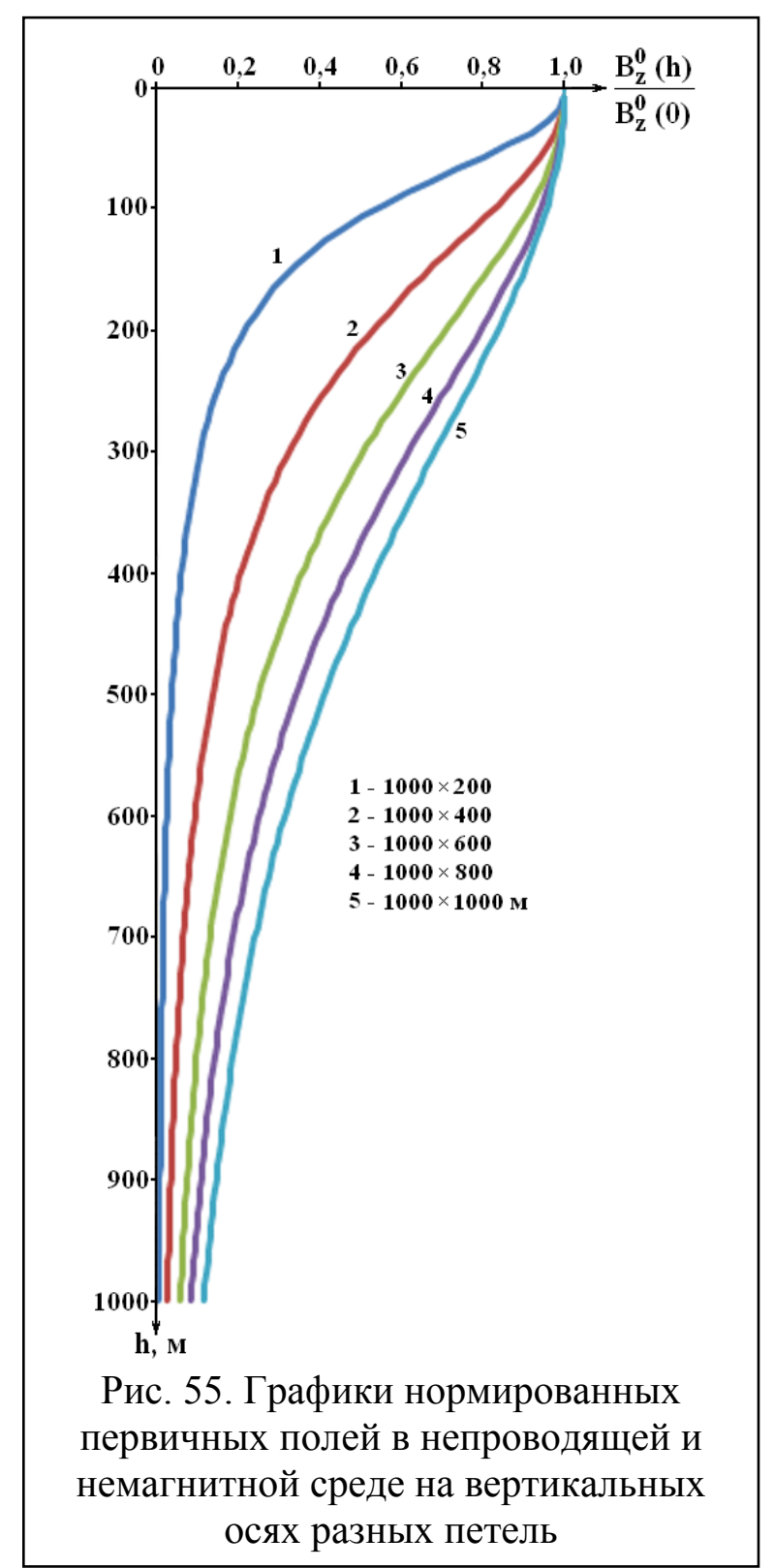

Самой универсальной и совмещающей в

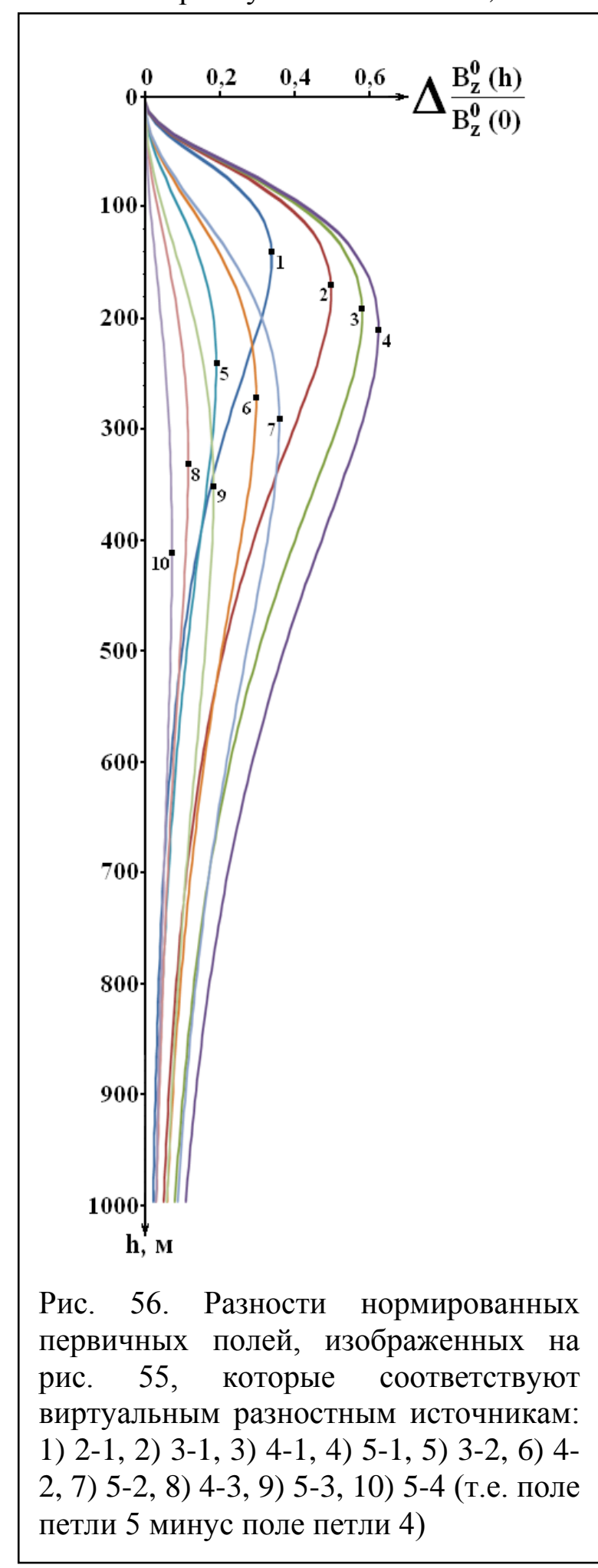

Рис. 56. Разности нормированных первичных полей, изображенных на рис. 55, которые соответствуют виртуальным разностным источникам: 1) $2-1$, 2) 3-1, 3) 4-1, 4) 5-1, 5) 3-2, 6) 42, 7) 5-2, 8) 4-3, 9) 5-3, 10) 5-4 (т.е. поле петли 5 минус поле петли 4)

\section{себе возможности профилирования и} зондирования, является методика, базирующаяся на применении набора прямоугольных петель $[45,48]$. У таких петель, разложенных вокруг изучаемого профиля, как показано на рис. 54, длина одинакова, а ширина различается. Чем ближе форма петли к квадрату, тем, очевидно, 
Ю.И. Блох

Теоретические основы комплексной магниторазведки

создаваемое ею подмагничивающее поле медленнее убывает с глубиной. На рис. 55 характер убывания проиллюстрирован графиками первичных полей в непроводящей и немагнитной среде на вертикальных осях разных петель, нормированных на значение в центре петли. Проводя измерения с набором таких петель, геофизики могут осуществлять послойное изучение геологических объектов.
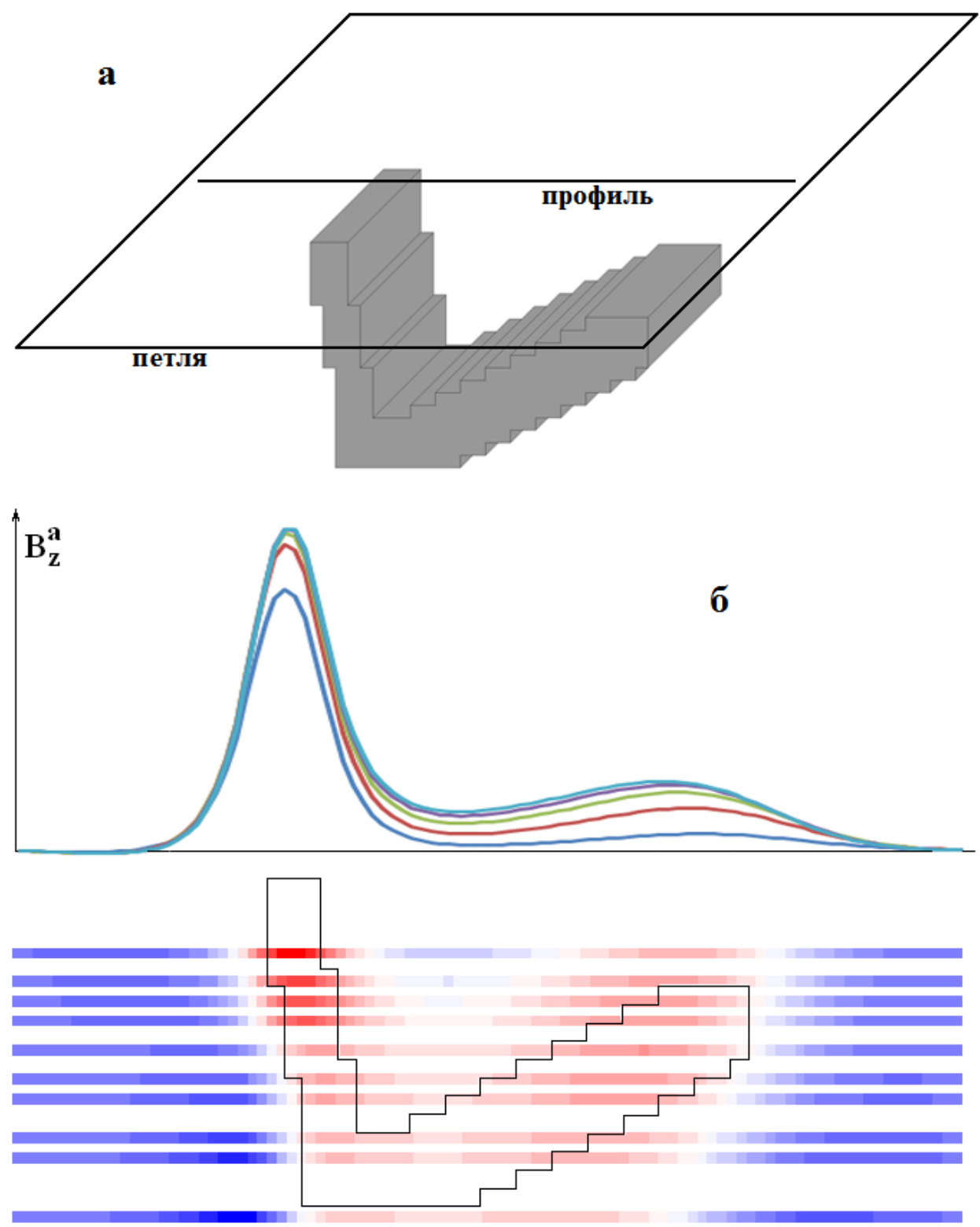

Рис. 57. Модельный пример томографической обработки многопетлевых наблюдений: а - теоретическая модель, наибольшая из намагничивающих ее петель и профиль наблюдений; б) аномалии от пяти намагничивающих петель и результаты их томографической обработки на вертикальном разрезе

Дело в том, что, вычисляя разности между наблюдениями в разных петлях, каждое из которых нормировано на значение того нормального поля, которое создавала соответствующая петля в пункте наблюдения, можно добиваться того же эффекта, который в МИП создавался инструментально, путем раскладки многовитковых петель. Другими словами, разности между нормированными значениями эквивалентны результатам измерений в петлях, компенсирующих нормальное поле в точке наблюдений и имеющих максимум на некоторой глубине. В 
современных условиях, когда существуют дешевые и точные методы определения формы петель, разложенных на местности, этот прием позволяет значительно сократить трудозатраты на смоточно-размоточные работы. На рис. 56 представлены графики разностей нормированных первичных полей, изображенных на рис. 55. Эти поля фактически соответствуют виртуальным разностным источникам, которые продуцируют разностные (редуцированные) аномалии. Обратим внимание на то, что их максимумы относятся к разным глубинам: в данном случае от 140 до 410 м, причем значения в экстремумах довольно существенно различаются.

Отмеченные закономерности могут быть положены в основу разнообразных томографических алгоритмов обработки результатов индукционных зондирований. Томография (в дословном переводе с греческого) значит «изображение слоев», и самый простой из томографических алгоритмов применительно к решаемой задаче сводится к следующему.

1. По формулам (2.7)-(2.9) производятся вычисления первичных полей прямоугольных петель, примененных для зондирования, на их вертикальных осях в непроводящей и немагнитной среде. Эти поля для каждой из петель нормируются на соответствующие значения в центрах петель на дневной поверхности.

2. Полученные нормированные поля всех петель вычитаются друг из друга всеми возможными способами по правилу: из полей больших петель - поля меньших. В результате для зондирования из $\mathrm{n}$ петель будут получены $\mathrm{n}(\mathrm{n}-1) / 2$ разностей.

3. Для каждой из разностей определяется глубина максимума и значение в нем. Понятно, что для горизонтальных плоских петель все это может быть сведено к простым формулам, но для реальных петель следует учитывать геометрию как можно точнее.

4. Для построения вертикального разреза в каждой из точек съемки аналогично вычисляются полученные разностные аномалии в долях нормального поля соответствующих петель в этих точках. Каждая из разностных аномалий делится на определенное значение в максимуме соответствующей разности теоретических нормированных первичных полей, а результат относится под данную точку профиля на глубину, равную глубине этого максимума. В итоге на разрезе появятся n(n-1)/2 уровней отнесения, по которым можно примерно судить об источниках.

Рассмотрим теоретический пример, демонстрирующий возможности алгоритма. Для 3D модели синформы были решены соответствующие прямые задачи в прямоугольных петлях, перечисленных на рис. 55. При этом магнитная восприимчивость модели считалась равной 1 СИ, и в процессе решения прямых задач, естественно, учитывались и неоднородность намагничивающего поля каждой из петель, и эффект размагничивания. Полученные аномалии в долях нормального поля в каждой из точек были обработаны по описанному алгоритму, и результаты обработки представлены на рис. 57, при этом по аномальным значениям в 5 петлях созданы изображения 10 слоев (уровней отнесения) в интервале глубин от 140 до 410 м.

Как видно, несмотря на простоту алгоритма обработки, результаты его применения оказались достаточно эффективными. Подчеркнем еще раз: при этом не использовалась никакая априорная информация, не задавалась модель, не решалась обратная задача. Стоит также отметить, что изучаемый объект был выбран сопоставимым по размерам с применяемыми петлями, то есть профиль наблюдения был относительно коротким, но, несмотря на это, глубокие части модели отразились на полученном разрезе вполне неплохо.

Таким образом, комплексная магниторазведка обладает серьезными возможностями томографической обработки результатов индукционных зондирований, при которых геофизики могут изучать сложные по морфологии геологические образования. Результаты подобной простой обработки весьма ценны для создания стартовой интерпретационной модели с целью ее дальнейшей оптимизации. При этом, естественно, указанные томографические возможности могут также конструктивно реализоваться в методах решения соответствующих обратных задач.

При решении ряда задач данная методика может существенно упрощаться. Например, в Якутии при поисках кимберлитовых трубок существенные помехи создают перекрывающие и крайне неоднородные по магнитным свойствам породы трапповой формации. Чтобы ослабить 
Ю.И. Блох

их влияние, достаточно ограничиться измерениями в двух петлях. Одна из петель должна быть близка по форме к квадратной и подмагничивать как глубокие части разреза, так и его верхнюю часть. Другая же, прямоугольная должна по возможности подмагничивать лишь траппы. Тогда, вычитая нормированные аномалии в меньшей петле из аномалий в большой петле, мы можем значительно ослаблять влияние траппов.

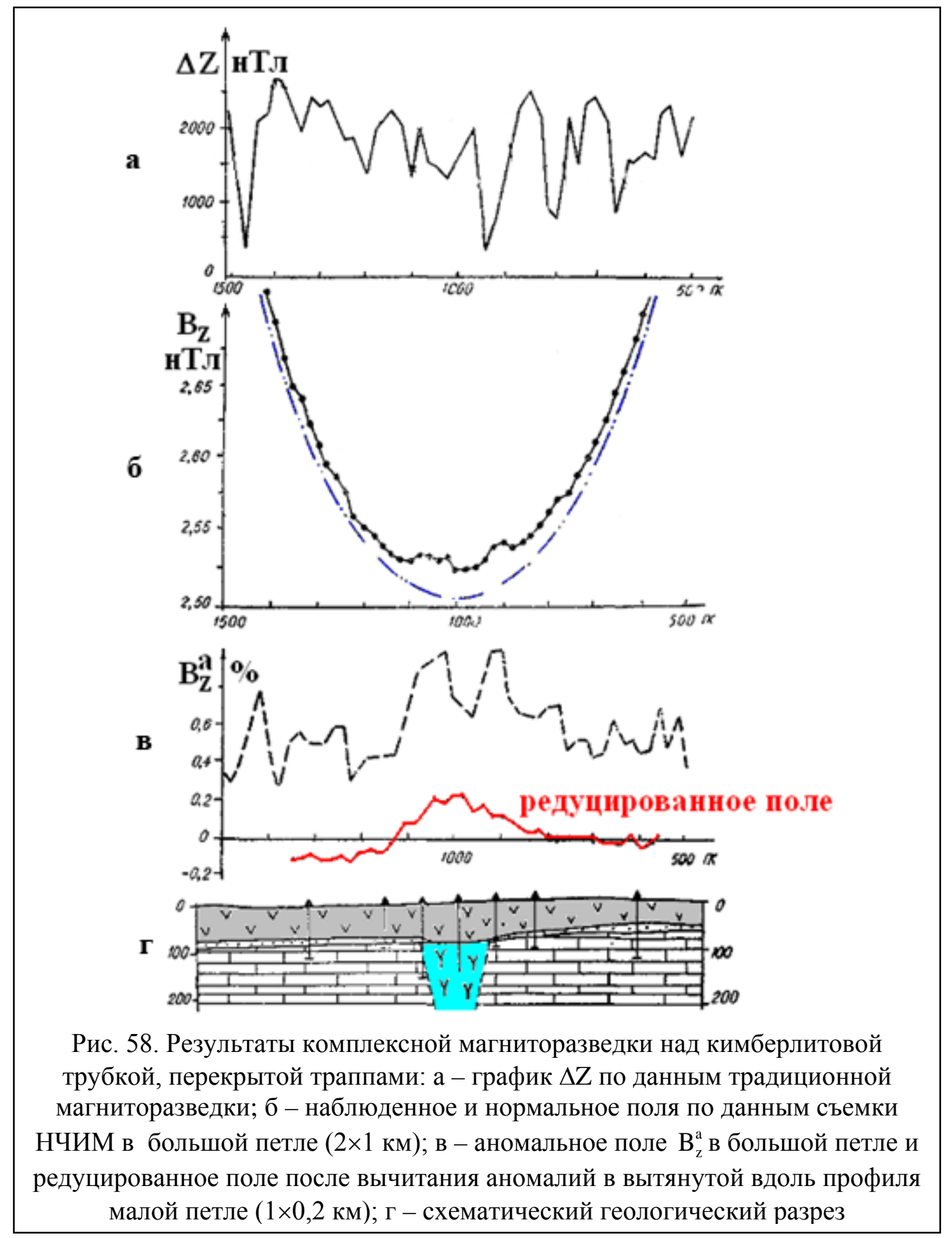

Проиллюстрируем эффективность применения упрощенной методики на практическом примере [48]. На рис. 58 представлены результаты комплексной магниторазведки над кимберлитовой трубкой, перекрытой слоем неоднородных пород трапповой формации мощностью около 100 м. Как видно, аномалии $\Delta \mathrm{Z}$ по данным традиционной магниторазведки на этом участке никак не выявляют кимберлитовую трубку. Аномалии $\mathrm{B}_{\mathrm{z}}^{\mathrm{a}}$ в большой петле 
размерами $2 \times 1$ км по данным НЧИМ в некоторой степени проявляют трубку за счет подавления влияния естественной остаточной намагниченности траппов. Однако наиболее эффективно она выявляется по редуцированному полю после вычитания аномалий в вытянутой вдоль профиля малой петле размерами $1 \times 0,2$ км. Обратим внимание, что соотношение сигнал/помеха в редуцированном поле почти на порядок превышает таковое для аномалий НЧИМ в большой петле, тогда как по сравнению с данными традиционной магниторазведки возрастание соотношения сигнал/помеха составляет почти два порядка.

Для упрощения интерпретации данных магнитного зондирования с соосными квадратными компенсирующими рамками над элементарными моделями В.Д. Стадухиным были разработаны специальные номограммы, которые можно найти в его методическом руководстве 1982 г. [170]. В настоящее время интерпретировать результаты подобных исследований можно без особых проблем с помощью компьютерного моделирования.

Еще одна возможность НЧИМ и МИП связана с управлением ориентацией векторов первичного поля в разных частях пространства. Это, в частности, позволяет изучать анизотропию магнитной восприимчивости рудных тел пластовой формы.

Выше, в 18 уже отмечалось, что задача определения компонент тензора магнитной восприимчивости возникает, как правило, тогда, когда в результате проведенных геологических и геофизических работ местоположение и форма изучаемого объекта уже достаточно хорошо известны и требуется изучить распределение в нем полезных компонент руды.

Для этого НЧИМ могут применяться в двух вариантах. В первом (более трудоемком) варианте измерения проводят последовательно в поле двух источников первичного поля. Один из них создает в объеме изучаемого объекта, поле, направление которого примерно совпадает с направлением полосчатости, а другой создает в том же объеме поле, ориентированное примерно перпендикулярно к ней. Если объект является, например, вертикальным пластом, то продольное поле в нем можно создать незаземленной петлей, разложенной на поверхности земли симметрично относительно пласта. Поперечное поле в этом случае можно создать длинным кабелем, разложенным вдоль простирания пласта над его верхней кромкой. Измерения аномалий индукции можно проводить как на поверхности земли, так и в

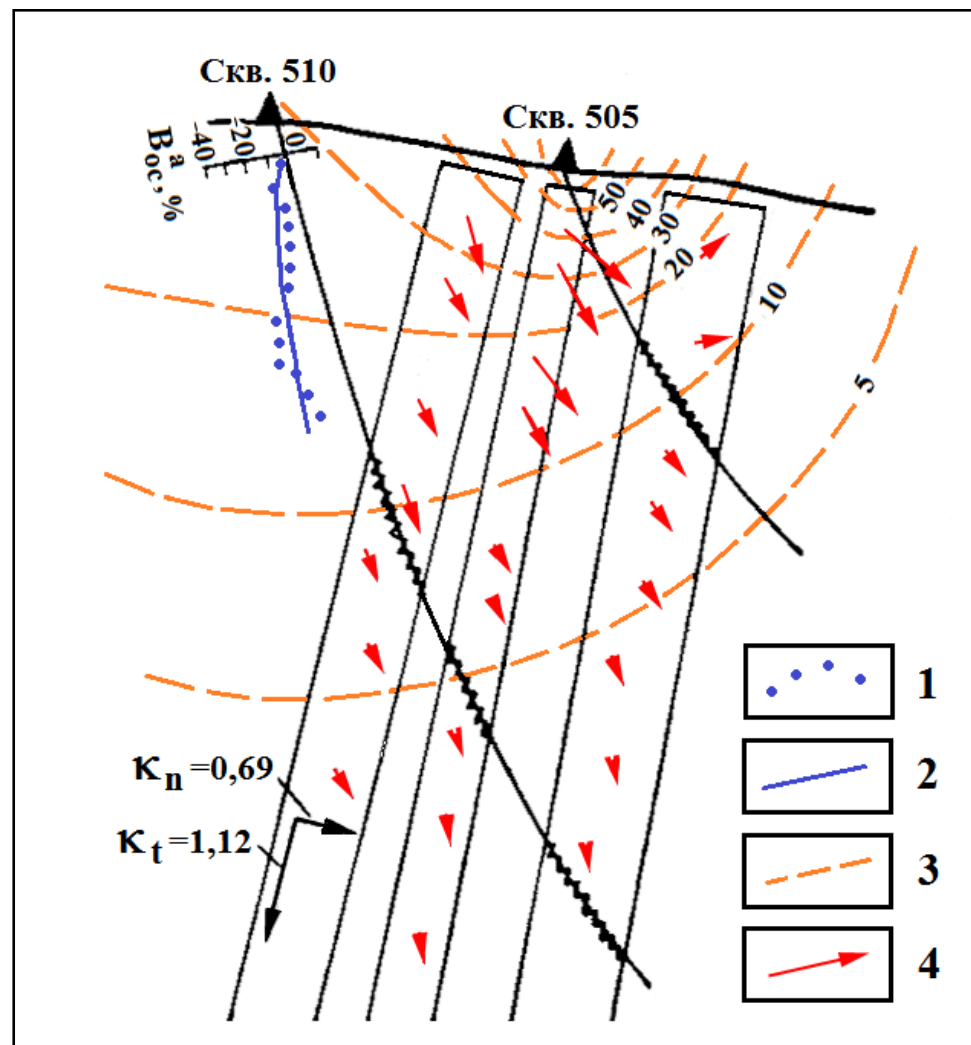

Рис. 59. Результаты определения компонент тензора магнитной восприимчивости пластов железистых кварцитов по данным скважинной комплексной магниторазведки: 1 - график наблюденного поля $\mathrm{B}_{\mathrm{oc}}^{\mathrm{a}}$ по данным скважинных наблюдений НЧИМ: 2 график подобранного поля анизотропной модели; 3 - изолинии модуля напряженности первичного поля петли; 4 - векторы первичного поля скважинах, в т.ч. неподсекших изучаемый объект.

По второму варианту измерения аномалий индукции проводят в поле одного источника, первичное поле которого ориентируют под углом к напластованию, создавая тем самым и продольное и поперечное намагничение объекта. Оптимальной для работы по второму 
варианту является ориентировка первичного поля источника под углом $\sim 45^{\circ}$ по отношению к напластованию, но вариации этого угла допустимы в весьма широких пределах. В качестве такого источника для вертикального пласта, например, может быть использована асимметрично расположенная незаземленная петля или длинный кабель, параллельный простиранию пласта, но не лежащий в плоскости его симметрии. Аномалии индукции при этом содержат информацию как о продольной, так и о поперечной компонентах тензора к, а разделение их влияний возможно на основе решения соответствующей линейной обратной задачи [Приложение]. Для перехода от кажущихся компонент, получаемых в результате решения этой системы, к истинным, надо путем моделирования учесть размагничивание объекта и ввести соответствующие поправки.

В качестве примера приведем результаты определения компонент тензора магнитной восприимчивости пластов железистых кварцитов на месторождении Тарыннах в Южной Якутии [45]. По данным бурения с учетом результатов наземных измерений НЧИМ в симметрично расположенной петле был построен разрез участка месторождения и определены продольные компоненты тензора магнитной восприимчивости для каждого из пластов. Затем в одной из скважин вне рудных подсечений были проведены измерения аномалий осевой компоненты поля $\mathrm{B}_{\text {ос }}^{\mathrm{a}}$ другой петли, одна из сторон которой располагалась над рудными телами (см. рис. 59). Моделирование скважинных результатов в рамках гипотезы об изотропности пластов при тех величинах их магнитной восприимчивости, которые были найдены по наземным данным, не дало совпадения с исходными данными. Удовлетворительные результаты получились только после перехода к модели анизотропных пластов. При этом средние компоненты тензора анизотропии магнитной восприимчивости оказались следующими: $\kappa_{\mathrm{t}}=1,12$ СИ и $\kappa_{\mathrm{n}}=0,69$ СИ. Эти параметры крайне важны при подсчете запасов месторождения по геофизическим данным, и их знание позволяет сделать его более точным.

\section{§ 21. Модель эллиптического цилиндра в комплексной магниторазведке}

В предыдущих параграфах было продемонстрировано, что комплексная магниторазведка наиболее эффективна, когда выполняется в многочастотных и многопетлевых модификациях. Однако на практике наиболее часто применяется экономичная съемка на единственной, достаточно низкой частоте в поле единственного источника: как правило, в форме прямоугольной петли, реже в поле длинного кабеля. Еще реже используются дипольные источники подмагничивающего поля. Соответственно, теоретическим основам интерпретации данных для самой распространенной однопетлевой и одночастотной методики следует уделить наибольшее внимание. Несмотря на упрощенность, она позволяет для довольно широкого класса геологических объектов определять форму и среднюю магнитную восприимчивость рудных тел, при этом, гораздо более представительную, нежели по измерениям на образцах. Далее же, зная магнитную восприимчивость и вычислив индуктивную намагниченность изучаемого объекта в земном магнитном поле, по данным традиционной магниторазведки можно определить величину и направление вектора остаточной намагниченности трехмерных тел. Для двумерных тел определяется проекция этого вектора на вертикальную плоскость, проходящую через интерпретационный профиль.

Естественно, подобные определения магнитных свойств возможны для таких классов железорудных объектов, для которых доказаны теоремы единственности решения соответствующих обратных задач. С точки зрения практики, наиболее важным из них является параметрический класс произвольных многоугольников (плоская задача), где, как доказал В.Н. Страхов, имеет место единственность даже при одном положении намагничивающего источника [178]. При малых значениях магнитной восприимчивости, то есть без учета размагничивания, слабая единственность для одного положения намагничивающего кабеля была установлена В.Н. Страховым для круговых цилиндров [178], а А.И. Лучицким - для эллиптических [124]. 
Вообще говоря, эллипсоиды и эллиптические цилиндры зачастую удобны для аппроксимации геологических объектов, но их применение долгое время было крайне ограниченным. Дело в том, что в магниторазведке бытовало мнение, что для эллиптических тел определяется только положение точек фокусов, а величину их осей принципиально можно определить только при известных значениях интенсивности намагничения. Однако, это утверждение справедливо не всегда. Если эллиптическое тело имеет лишь индуктивную намагниченность в известном поле, направление которого отличается от направления осей тела, как это зачастую имеет место в МИП и НЧИМ, то в результате решения обратной задачи можно определить и его размеры и интенсивность намагничения, а, следовательно, и магнитную восприимчивость.

Первым, кто обратил внимание на такую возможность, стал Д.П. Зидаров. Он доказал в 1968 г. следующую теорему: если намагничивающее поле однородно и не совпадает по направлению ни с одним из направлений полуосей индуктивно намагниченного эллипсоида, то не существует другого эллипсоида, индуктивно намагниченного в том же поле и имеющего точно такое же внешнее аномальное поле [88, с. 90-91]. Эту теорему Д.П. Зидаров доказал методом от противного, что, соответственно, не давало возможности строить на ее базе методику интерпретации. В 1981 г. конструктивное доказательство слабой единственности для эллиптического цилиндра удалось получить автору [25], и на его базе (с учетом перехода к системе единиц СИ) далее будет излагаться разработанная методика интерпретации. Несколько позже доказательство данного факта независимо получил, выведя явное выражение поля эллипса в комплексных координатах, П.С. Мартышко [129].

Итак, пусть эллиптический цилиндр с полуосями a и b $(\mathrm{a}>\mathrm{b})$, имеющий магнитную восприимчивость $\kappa$, расположен в однородном первичном поле с индукцией $\overline{\mathrm{B}^{\text {прв }}}$, как показано на рис. 60. Угол между осью Ох и длинной осью цилиндра, отсчитываемый против часовой стрелки, обозначим через $\alpha$. Острый угол между длинной осью и направлением первичного поля обозначим через $\varphi$. Разложим первичное поле $\overline{\mathrm{B}^{п в}}$ на составляющие, параллельные осям цилиндра, и воспользуемся известными выражениями для его индуктивной намагниченности. Составляющая $\mathrm{I}_{\mathrm{a}}$, параллельная оси 2a, равна

$$
I_{a}=\frac{\kappa(a+b) B^{\text {прв }} \cos \varphi}{\mu_{0}(a+b+\kappa b)} .
$$

Другая составляющая $\mathrm{I}_{\mathrm{b}}$ - соответственно

$$
\mathrm{I}_{\mathrm{b}}=\frac{\kappa(\mathrm{a}+\mathrm{b}) \mathrm{B}^{\text {прв }} \sin \varphi}{\mu_{0}(\mathrm{a}+\mathrm{b}+\kappa \mathrm{a})} .
$$

Учитывая соотношение между магнитной восприимчивостью и магнитной проницаемостью $\mu=1+\kappa$, получим следующее выражение для модуля вектора намагниченности $|\overline{\mathrm{I}}|$ :

$$
|\overline{\mathrm{I}}|=\frac{(\mu-1)(\mathrm{a}+\mathrm{b}) \mathrm{B}^{\text {прв }}}{\mu_{0}} \sqrt{\frac{\cos ^{2} \varphi}{(\mathrm{a}+\mu \mathrm{b})^{2}}+\frac{\sin ^{2} \varphi}{(\mathrm{b}+\mu \mathrm{a})^{2}}} .
$$

Нулевой комплексный момент представляет собой произведение намагниченности на площадь поперечного сечения объекта [39]. Так как площадь эллипса равна $\mathrm{S}=\pi \mathrm{ab}$, то модуль нулевого момента можно записать в виде

$$
\left|M_{0}\right|=\frac{\pi \mathrm{ab}(\mu-1)(\mathrm{a}+\mathrm{b}) \mathrm{B}^{\text {прв }}}{\mu_{0}} \sqrt{\frac{\cos ^{2} \varphi}{(a+\mu b)^{2}}+\frac{\sin ^{2} \varphi}{(b+\mu a)^{2}}} .
$$


Ю.И. Блох

Острый угол $\theta$ между вектором намагниченности и длинной осью цилиндра, как следует из (21.1) и (21.2), связан с параметрами эллипса соотношением

$$
\frac{\operatorname{tg} \theta}{\operatorname{tg} \varphi}=\frac{a+\mu b}{b+\mu a} .
$$

Первый комплексный момент представляет собой произведение нулевого момента на аффикс центра масс, а второй момент относительно центра масс $\mathrm{u}_{\mathrm{m}}$ для эллипса равен [39]

$$
\mathrm{M}_{2}\left(\mathrm{u}_{\mathrm{m}}\right)=\mathrm{M}_{0} \frac{\mathrm{a}^{2}-\mathrm{b}^{2}}{4} \mathrm{e}^{2 \mathrm{i} \alpha} .
$$

Этими соотношениями можно воспользоваться при решении обратной задачи и построения квазиэквивалента. Вычислив с помощью любого из известных методов нулевой, первый и второй моменты по наблюденному полю, определим координаты центра масс $\mathrm{u}_{\mathrm{m}}=\mathrm{x}_{\mathrm{m}}+\mathrm{iz}$ и угол $\alpha$ по формулам:

$$
\begin{gathered}
\mathrm{u}_{\mathrm{m}}=\frac{\mathrm{M}_{1}(0)}{\mathrm{M}_{0}}, \\
\alpha=\frac{1}{2} \arg \frac{\mathrm{M}_{2}\left(\mathrm{u}_{\mathrm{m}}\right)}{\mathrm{M}_{0}} .
\end{gathered}
$$

Далее, решив систему уравнений (21.4)-(21.6), найдем параметры a, b и $\mu$. Для упрощения решения выразим $\mathrm{a}+\mu \mathrm{b}$ из (21.5) и подставим в (21.4). В результате система примет вид

$$
\begin{gathered}
\frac{\mathrm{ab}(\mathrm{a}+\mathrm{b})(\mu-1)}{\mathrm{b}+\mu \mathrm{a}}=\frac{\mu_{0} \sin \theta}{\pi \sin \varphi}\left|\frac{\mathrm{M}_{0}}{\mathrm{~B}^{\mathrm{npB}}}\right|, \\
\frac{\mathrm{a}+\mu \mathrm{b}}{\mathrm{b}+\mu \mathrm{a}}=\frac{\operatorname{tg} \theta}{\operatorname{tg} \varphi}, \\
\mathrm{a}^{2}-\mathrm{b}^{2}=4\left|\frac{\mathrm{M}_{2}\left(\mathrm{u}_{\mathrm{m}}\right)}{\mathrm{M}_{0}}\right| .
\end{gathered}
$$

Введем обозначения

$$
\mathrm{A}=\frac{\mu_{0} \sin \theta}{\pi \sin \varphi}\left|\frac{\mathrm{M}_{0}}{\mathrm{H}^{\mathrm{\Pi p \textrm {B }}}}\right|, \mathrm{B}=\frac{\operatorname{tg} \theta}{\operatorname{tg} \varphi}, \mathrm{C}=4\left|\frac{\mathrm{M}_{2}\left(\mathrm{u}_{\mathrm{m}}\right)}{\mathrm{M}_{0}}\right|, \quad \mathrm{D}=\frac{\mathrm{A}}{\mathrm{C}(1-\mathrm{B})} .
$$

Тогда решение системы будет следующим:

$$
a=\sqrt{\frac{C}{2}\left(\frac{1+2 \mathrm{D}}{\sqrt{1+4 \mathrm{D}}}+1\right)}, \mathrm{b}=\sqrt{\frac{\mathrm{C}}{2}\left(\frac{1+2 \mathrm{D}}{\sqrt{1+4 \mathrm{D}}}-1\right)}, \mu=\frac{\mathrm{a}-\mathrm{bB}}{\mathrm{aB}-\mathrm{b}} .
$$

Эти формулы не только доказывают единственность решения рассматриваемой обратной задачи, но и фактически представляют собой алгоритм ее решения. Алгоритм применим всегда, когда первичное поле не направлено по направлению какой-либо из осей эллипса, иначе соотношение (21.5) становится неопределенным.

Итак, в результате интерпретации данных НЧИМ либо МИП можно определить геометрические параметры эллиптического цилиндра и его магнитную восприимчивость. Далее по данным обычной магниторазведки определяется проекция вектора его остаточной намагниченности на вертикальную плоскость, проходящую через интерпретационный профиль. Для этого разложим магнитный момент эллиптического цилиндра, найденный по данным

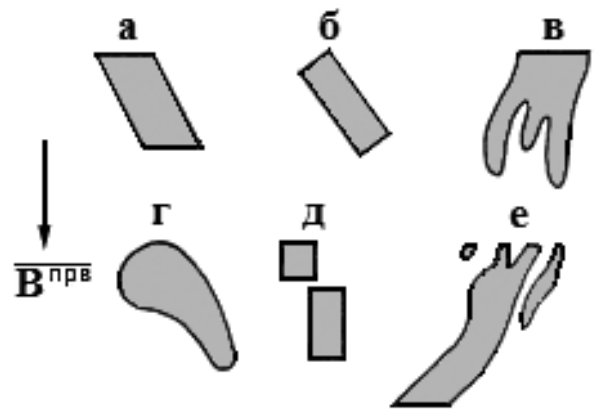

Рис. 61. Модели, использованные в численном эксперименте обычной магниторазведки, на компоненты $\mathrm{M}_{\mathrm{a}}$ и $\mathrm{M}_{\mathrm{b}}$, параллельные соответствующим осям цилиндра. Если острый угол между проекцией вектора индукции нормального поля Земли на вертикальную плоскость, проходящую через 
Ю.И. Блох

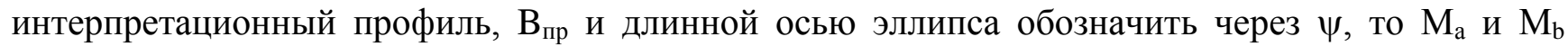
выразятся через параметры эллипса следующим образом:

$$
\begin{aligned}
& M_{a}=\frac{\pi a b(a+b)\left(I_{n a}+\kappa B_{\text {пр }} \cos \psi / \mu_{0}\right)}{a+\mu b}, \\
& M_{b}=\frac{\pi a b(a+b)\left(I_{n b}+\kappa B_{n p} \sin \psi / \mu_{0}\right)}{b+\mu a},
\end{aligned}
$$

где $\mathrm{I}_{\mathrm{na}}$ и $\mathrm{I}_{\mathrm{nb}}$ - проекции вектора остаточной намагниченности на оси эллипса. Из соотношений (21.12) несложно определить $\mathrm{I}_{\text {na }}$ и $\mathrm{I}_{\mathrm{nb}}$, которые при необходимости можно пересчитать в горизонтальную и вертикальную составляющие.

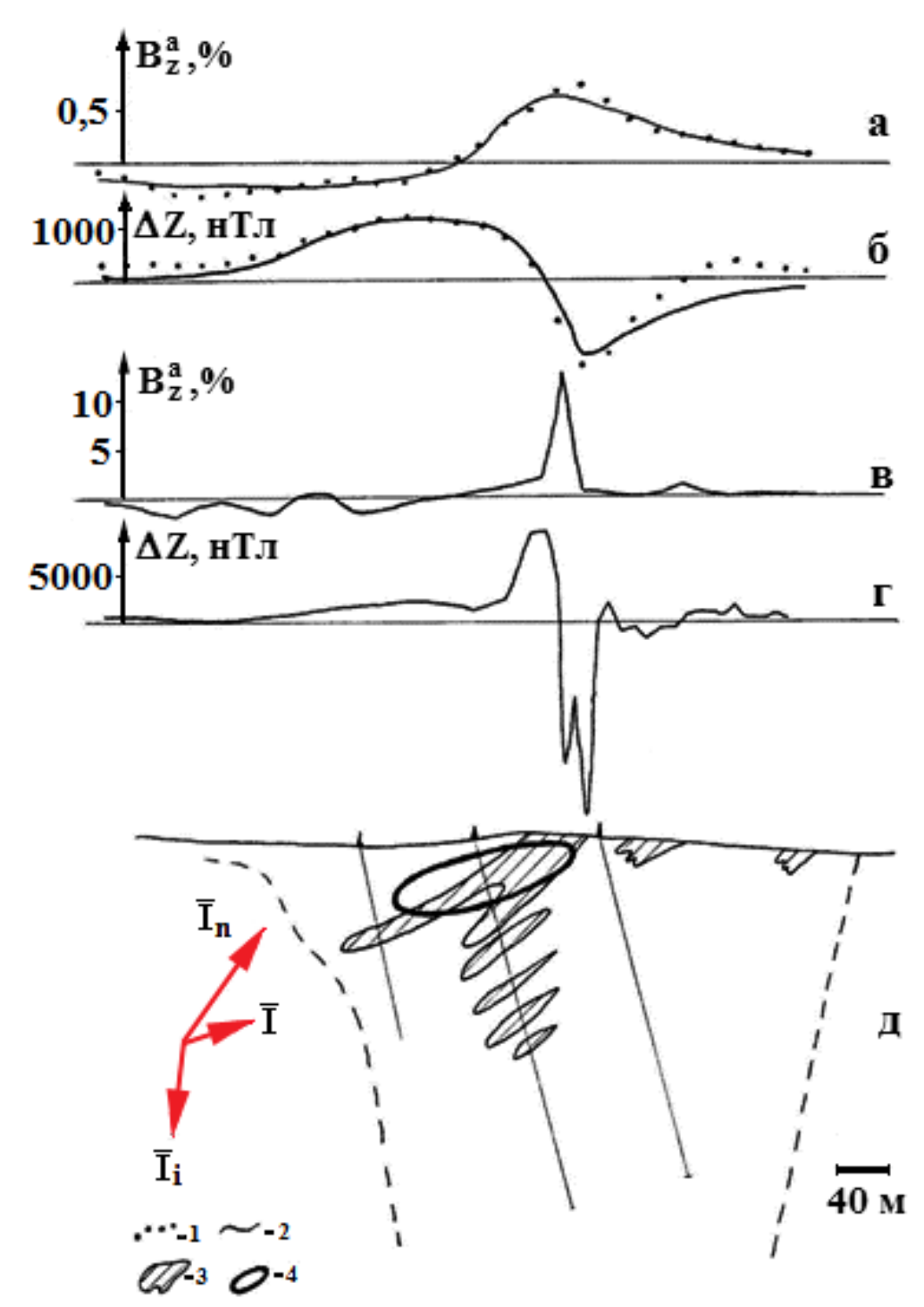

Рис. 62. Результаты интерпретации полевых материалов на магномагнетитовом рудопроявлении в Среднем Приангарье: а - подбор аномалии $\mathrm{B}_{\mathrm{z}}^{\mathrm{a}}$, продолженной на высоту 40 м; 6 - то же для аномалии $\Delta \mathrm{Z}$; в - наблюденная аномалия $\mathrm{B}_{z}^{\mathrm{a}}$;

г - наблюденная аномалия $\Delta \mathrm{Z}$; д - геологический разрез с результатами интерпретации; 1 - аналитически продолженные аномалии для а и б; 2 - подобранные аномалии для а и б; 3 - рудные тела по геологическим данным; 4 - квазиэквивалентный эллипс

Таким образом, в результате комплексной интерпретации данных метода незаземленной петли и магниторазведки можно однозначно определить геометрические параметры и магнитные свойства намагничивающегося эллиптического цилиндра. 
Несмотря на то, что эллиптическая форма характерна для скарновых магнетитовых рудных тел, многие месторождения отличаются более сложным строением. В этой связи необходимо оценить возможность использования модели эллиптического цилиндра при интерпретации аномалий над телами разнообразной формы. Для этого был проведен численный эксперимент, заключавшийся в вычислении первых гармонических моментов моделей, показанных на рис. 61 и намагниченных в однородном вертикальном поле. Магнитная восприимчивость моделей принята большой, чтобы неоднородность их намагничения под влиянием эффекта размагничивания, мешающая применению простой эллиптической модели, была бы значительной. Полученные значения, сведенные в табл. 9, были использованы для оценки магнитной восприимчивости и площади поперечного сечения эллиптического цилиндра S. Результаты опробования алгоритма свидетельствуют о возможности оценки с его помощью параметров достаточно сложных объектов. При этом, естественно, результаты тем лучше, чем ближе форма поперечного сечения объекта к эллипсу, а наиболее неблагоприятным оказался случай двух изолированных объектов.

Таблица 9.

Результаты численного эксперимента с моделями, изображенными на рис. 61.

\begin{tabular}{|c|c|c|c|c|}
\hline \multirow{2}{*}{ Модель } & \multicolumn{2}{|c|}{$\begin{array}{c}\text { Магнитная восприимчивость } к, \\
\text { ед. СИ }\end{array}$} & \multicolumn{2}{c|}{$\begin{array}{c}\text { Площадь поперечного сечения S, } \\
\text { усл. ед. }\end{array}$} \\
\cline { 2 - 5 } & Истинная & Вычисленная & Истинная & Вычисленная \\
\hline $\mathrm{a}$ & 6.283 & 4.901 & 160,0 & 161,4 \\
\hline б & 6.283 & 5.027 & 108,0 & 120,1 \\
\hline в & 6.283 & 5.781 & 192,0 & 193,8 \\
\hline г & 6.283 & 3.267 & 180,0 & 240,8 \\
\hline д & 6.283 & 1.131 & 108,0 & 268,5 \\
\hline е & 1.257 & 0.628 & 260,0 & 422,2 \\
\hline
\end{tabular}

На рис. 62 приведены результаты применения предложенной методики на одном из магномагнетитовых рудопроявлений Среднего Приангарья. Над ним наблюдается положительная аномалия $\mathrm{B}_{\mathrm{z}}^{\mathrm{a}}$ метода незаземленной петли амплитудой $12 \%$ от первичного поля и знакопеременная аномалия $\Delta \mathrm{Z}$, отрицательный экстремум которой достигает -19300 нТл. Это свидетельствует о значительном влиянии остаточной намагниченности. Аномалии $\mathrm{B}_{\mathrm{z}}^{\mathrm{a}}$ и $\Delta \mathrm{Z}$ были аналитически продолжены вверх на высоту 40 м и на этом уровне подобраны полем одной пластинки, а полученные комплексные моменты обработаны с учетом известного по данным каротажа магнитной восприимчивости значения к. В результате определены геометрические параметры эллиптического цилиндра и компоненты векторов его индуктивной и остаточной намагниченности.

Естественно, возможности рассмотренной модели вовсе не исчерпываются изложенными в данном параграфе приложениями. Так, П.С Мартышко и В.А. Пьянков с успехом применяли модель эллипса, намагничивающегося не по направлению его осей, в МВМ для интерпретации поля $\mathrm{S}_{\mathrm{q}}$ вариаций [132], что расширило область применения как этого метода, так и комплексной магниторазведки в целом.

\section{§ 22. Модели крутопадающих пластов в комплексной магниторазведке}

Как отмечалось выше, основные мировые запасы магнетитовых руд связаны с месторождениями метаморфогенной железорудной формации, представленной обычно магнетитовыми и гематито-магнетитовыми кварцитами. Характерной для данной формации формой рудных тел являются крутопадающие пласты, а также пачки таких пластов. Аналогичная форма часто встречается и в месторождениях, принадлежащих к другим 
формациям. В связи с этим в комплексной магниторазведке наиболее часто встречаются модели уединенного пласта или пачки параллельных пластов.

Обратная задача комплексной магниторазведки для пачки параллельных крутопадающих пластов имеет единственное решение. Это вытекает из единственности определения верхней кромки пластов по особым точкам функций, описывающих аномальные поля. Угол падения пачки по данным НЧИМ или МИП также определяется однозначно, поскольку ориентировка первичного поля петли в месте расположения пачки известна - как правило, поле вертикально или близко к вертикальному. Таким образом, при известной геометрии объекта, его магнитные свойства могут быть определены путем решения линейной обратной задачи. Для данного случая, характерного тем, что изменение физических свойств исключительно латеральное, то есть от одного пласта к другому, линейная обратная задача имеет единственное решение [39].

Несмотря на единственность решения, рассматриваемая комплексная обратная задача весьма неустойчива из-за большого числа требующих определения параметров. Для одного пласта число параметров равно 8: две пары координат краев верхней пласта, угол его падения, магнитная восприимчивость и две компоненты вектора естественной остаточной намагниченности, характеризующие его проекцию на вертикальную плоскость, проходящую через интерпретационный профиль. Если $\mathrm{P}$ пластов объединены в пачку, то число определяемых параметров равно 7P+1, учитывая, что угол падения у всех пластов одинаков. На практике число пластов, объединенных в пачку, нередко составляет 15-20, так что при интерпретации требуется находить 100-150 и более параметров. Решение задач такой размерности исключительно в рамках метода подбора неустойчиво, поэтому эффективную методику интерпретации в рассматриваемой задаче можно строить лишь путем комплексирования различных интерпретационных методов, базирующихся на разных идеях извлечения информации.

Для модели пачки намагниченных крутопадающих пластов эффективная и естественно вытекающая из существа решаемой задачи методика интерпретации должна включать три этапа. На первом этапе с помощью хорошо разработанных методов определяются особые точки, характеризующие верхнюю кромку пачки. На втором этапе методом подбора по данным НЧИМ или МИП при закрепленных особых точках находятся угол падения пачки и магнитные восприимчивости входящих в нее пластов. Наконец, на третьем этапе по данным магниторазведки при опоре на параметры, найденные на первом и втором этапах, методом подбора определяются компоненты вектора естественной остаточной намагниченности. Рассмотрим основные черты каждого из этапов.

Локализация особых точек функций, аномальные поля, может осуществляться по-разному в зависимости от особенностей геологического строения района работ. Для этого наиболее целесообразно использовать совместно магниторазведки, НЧИМ или МИП. При небольшой глубине залегания верхней кромки пачки вплоть до 10-20 м могут быть использованы даже простейшие приемы, основанные

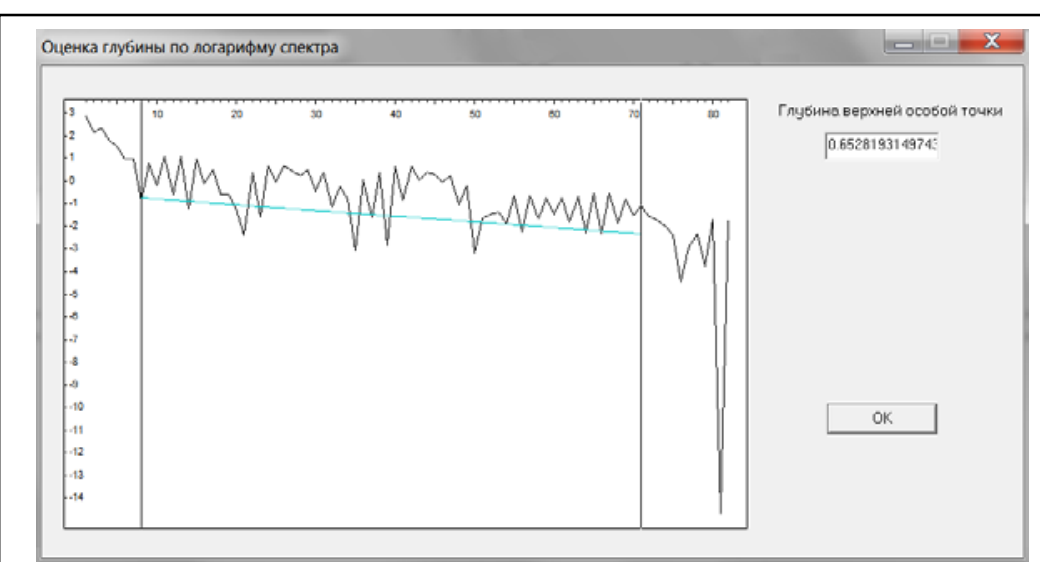

Рис. 63. Оценка глубины верхней особой точки в интегрированной системе СИНГУЛЯР идентификации точек наибольшего горизонтального градиента $\mathrm{B}_{\mathrm{z}}^{\mathrm{a}}$ и $\Delta \mathrm{Z}$ и применении способа касательных. Он, как показано выше, даже в условиях значительного влияния размагничивания не приводит к заглублению верхней кромки более чем на 10-20 \%, к тому же эффект заглубления можно корректировать, используя рис. 46. 
Ю.И. Блох

Теоретические основы комплексной магниторазведки

Более точные результаты, особенно, для источников с глубиной залегания, превышающей 20 м, могут достигаться с помощью интегрированной системы СИНГУЛЯР. Эта система была разработана в начале 1990-х годов автором совместно с Д.В. Каплуном и О.Н. Коняевым [50] и с тех пор продолжает непрерывно совершенствоваться. В ее основу положен интерактивный синтез результатов, получаемых разнородными методами, которые ранее были предложены В.Н. Страховым, В.М. Березкиным, Г.А. Трошковым и В.К. Ивановым. В отличие от большинства существующих программ для локализации особенностей, СИНГУЛЯР предоставляет, в частности, возможности локализации особых точек функций, характеризующих поля, которые измерены на неровном рельефе.

На рис. 63 и 64 показан пример применения системы СИНГУЛЯР на одном из профилей железорудного месторождения Тарыннах в Южной Якутии. Там с 10-метровым шагом по профилю были выполнены съемки обычной магниторазведкой и одним из наиболее популярных среди НЧИМ - методом незаземленной петли. Сначала по графику логарифма амплитудного спектра исходного поля оценивается глубина верхней особой точки - так называемый параметр Н. Для этого в соответствии с методикой, разработанной В.К. Ивановым, этот график интерактивно аппроксимируется прямой в пределах среднего интервала частот, и угол наклона этой прямой характеризует параметр Н. На рис. 63 видно, каким образом был выбран интервал для спектра поля $\mathrm{B}_{z}^{\mathrm{a}}$ и каким оказался результат. Глубина верхней особенности в шагах съемки оказалась равной 0,65 , то есть 6,5 м.

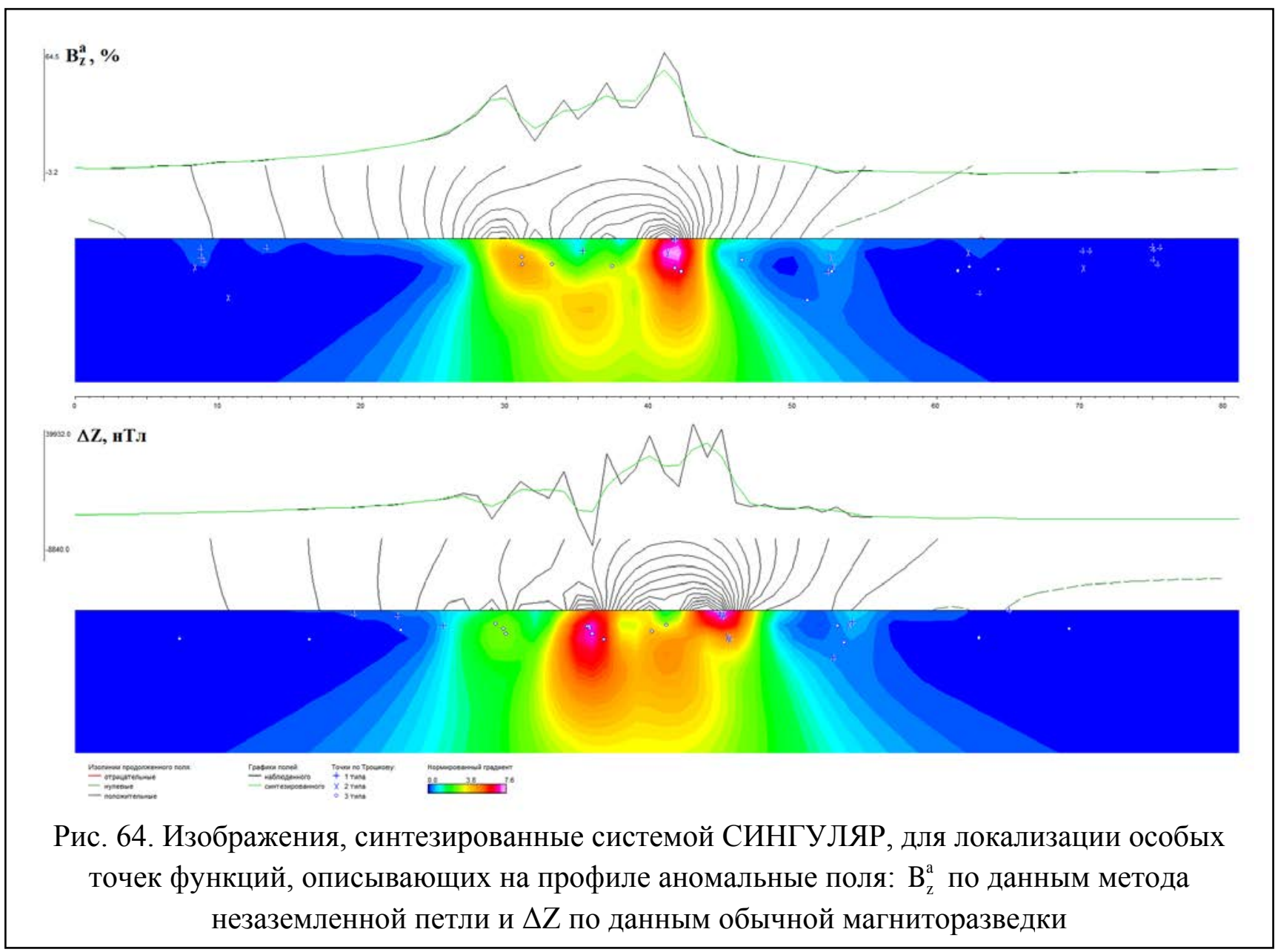

Далее с учетом найденного параметра Н система СИНГУЛЯР выполняет расчеты по трем наиболее хорошо зарекомендовавших себя на практике методам локализации особых точек, разработанным В.Н. Страховым, В.М. Березкиным и Г.А. Трошковым. Результаты вычислений синтезируются в оригинальном интерфейсе, дающим интерпретатору возможность осуществить 
синтез полученных данных и оценить координаты особенностей и их типы [39]. На рис. 64 сведены изображения, синтезированные системой СИНГУЛЯР, для локализации особых точек функций, описывающих на профиле аномальные поля $\mathrm{B}_{z}^{\mathrm{a}}$ по данным метода незаземленной петли и $\Delta \mathrm{Z}$ по данным традиционной магниторазведки. Там под графиками аномальных полей изображены вертикальные разрезы, где изолиниями показаны результаты аппроксимационного продолжения аномального поля по Страхову, растровая карта нормированного градиента поля по Березкину и значки, характеризующие местоположения и типы особых точек по Трошкову. Значком «+» отмечены вероятные вершины многоугольников, значком «х» - края тонких пластинок, а кружочками - центры локальных магнитных масс.

Как правило, наиболее устойчиво локализуются крайние особые точки, характеризующие самый левый и самый правый пласты в пачке. Тем самым глубина верхней кромки и видимая мощность пачки находятся практически всегда достаточно уверенно. Если же отдельные пласты по графикам $\mathrm{B}_{z}^{\mathrm{a}}$ и $\Delta \mathrm{Z}$ с привлечением результатов методов особых точек выделить не удается, эта часть пачки формально разделяется на ряд тонких соприкасающихся пропластков, для которых методом подбора определяется магнитная восприимчивость. После этого смежные пропластки с практически одинаковыми значениями $\kappa$ формально объединяются в один пласт. Когда пропластков много, для оценки их магнитных восприимчивостей целесообразно пользоваться методом регуляризации со стабилизатором в виде нормы искомого решения [39].

При локализации особых точек целесообразно привлекать также данные других геофизических методов, входящих в комплекс, в первую очередь, гравиразведки. Вместе с тем, необходимо иметь в виду, что особые точки функций, описывающих гравитационные и магнитные поля, могут не совпадать. При наличии в верхней части пластов окисленных руд гематитового и мартитового составов, отличающихся низкой магнитной восприимчивостью, особые точки, найденные по данным гравиразведки, окажутся расположенными выше, чем соответствующие особенности, локализованные по материалам традиционной магниторазведки, МИП и НЧИМ. Тем самым, анализируя локализованные особые точки, можно установить наличие зоны окисления и оценить ее мощность.

Итак, на первом этапе интерпретации пачка подразделяется на отдельные пласты с известным положением верхней кромки, на втором этапе по данным НЧИМ или МИП требуется найти угол падения пачки и магнитную восприимчивость каждого из пластов. Для этого наиболее просто и эффективно пользоваться методом квазирешений, минимизируя сумму квадратов отклонений наблюденной аномалии $\mathrm{B}_{\mathrm{z}}^{\mathrm{a}}$ от теоретической аномалии пачки пластов. Выше, в $§ 13$ был рассмотрен вопрос о взаимовлиянии сближенных объектов и показано, что, вообще говоря, они существенно изменяют намагниченность друг друга, так что, строго говоря, магнитное поле пачки не равно сумме полей каждого из пластов, входящих в пачку. Вместе с тем, анализ показал, что даже для соприкасающихся эллиптических цилиндров, вытянутых вдоль намагничивающего поля, коэффициент взаимовлияния мал. Если относительная вытянутость объектов $\mathrm{k} \rightarrow \infty$, коэффициент взаимовлияния стремится к единице, что видно на рис. 25. Эти данные показывают, что при подборе магнитных восприимчивостей продольно намагниченных пластов, их взаимным влиянием в определенной мере можно пренебрегать, не забывая, естественно, о том, что в результате такого подбора определяются не истинные значения магнитной восприимчивости, а кажущиеся, которые далее необходимо исправлять за влияние размагничивания и неоднородность намагничивающего поля петли.

В соответствии со сказанным, в качестве теоретической аномалии в методе незаземленной петли можно использовать магниторазведочные аномалии вертикально намагниченных пластов, что приводит к задаче вида:

$$
\Phi\left(\varphi, \kappa_{1}^{\prime}, \kappa_{2}^{\prime}, \ldots, \kappa_{\mathrm{p}}^{\prime}\right)=\sum_{\mathrm{k}=1}^{\mathrm{N}}\left[\mathrm{B}_{\mathrm{z}}^{\mathrm{a}}\left(\mathrm{x}_{\mathrm{k}}, \mathrm{z}_{\mathrm{k}}\right)-\sum_{\mathrm{i}=1}^{\mathrm{p}} \kappa_{\mathrm{i}}^{\prime} \mathrm{B}_{\mathrm{zi}}\left(\mathrm{x}_{\mathrm{k}}, \mathrm{z}_{\mathrm{k}}, \mathrm{x}_{\mathrm{li}}, \mathrm{z}_{\mathrm{li}}, \mathrm{x}_{\mathrm{pi}}, \mathrm{z}_{\mathrm{pi}}, \varphi\right)\right]^{2}=\min .
$$

Здесь $\mathrm{B}_{\mathrm{zi}}$ - аномалия вертикальной составляющей i-го пласта с единичной магнитной восприимчивостью в долях первичного поля петли в точке наблюдений с координатами $\mathrm{x}_{\mathrm{k}}, \mathrm{z}_{\mathrm{k}}$ 
(ось Ox направлено вправо, ось $\mathrm{Oz}$ - вверх); $\mathrm{B}_{z}^{\mathrm{a}}$ - наблюденная аномалия в той же точке; $\varphi-$ угол падения пачки, отсчитываемый от оси Ох по часовой стрелке и изменяющийся в пределах от 0 до $180^{\circ}$. Число точек наблюдения обозначено N, а число пластов - Р. Координаты $\mathrm{x}_{\mathrm{li}}$ и $\mathrm{z}_{\mathrm{li}}$ характеризуют левый угол і-го пласта, а х восприимчивость пласта.

$$
\begin{gathered}
\mathrm{B}_{\mathrm{zi}}\left(\mathrm{x}_{\mathrm{k}}, \mathrm{z}_{\mathrm{k}}, \mathrm{x}_{\mathrm{li}}, \mathrm{z}_{\mathrm{li}}, \mathrm{x}_{\mathrm{pi}}, \mathrm{z}_{\mathrm{pi}}, \varphi\right)=\frac{\sin (\varphi-\alpha)}{2 \pi}\left[\sin (\varphi+\alpha)\left(\operatorname{arctg} \frac{\mathrm{x}_{\mathrm{k}}-\mathrm{x}_{\mathrm{li}}}{\mathrm{z}_{\mathrm{k}}-\mathrm{z}_{\mathrm{li}}}-\operatorname{arctg} \frac{\mathrm{x}_{\mathrm{k}}-\mathrm{x}_{\mathrm{pi}}}{\mathrm{z}_{\mathrm{k}}-\mathrm{z}_{\mathrm{pi}}}\right)+\right. \\
\left.+\frac{\cos (\varphi+\alpha)}{2} \ln \frac{\left(\mathrm{z}_{\mathrm{k}}-\mathrm{z}_{\mathrm{li}}\right)^{2}+\left(\mathrm{x}_{\mathrm{k}}-\mathrm{x}_{\mathrm{li}}\right)^{2}}{\left(\mathrm{z}_{\mathrm{k}}-\mathrm{z}_{\mathrm{pi}}\right)^{2}+\left(\mathrm{x}_{\mathrm{k}}-\mathrm{x}_{\mathrm{pi}}\right)^{2}}\right],
\end{gathered}
$$

где $\alpha$ - угол наклона верхней кромки і-го пласта:

$$
\alpha=\operatorname{arctg} \frac{\mathrm{x}_{\mathrm{pi}}-\mathrm{x}_{\mathrm{li}}}{\mathrm{z}_{\mathrm{pi}}-\mathrm{z}_{\mathrm{li}}} .
$$

В частном случае при горизонтальном расположении верхней кромки пласта $\alpha=0, a z_{\mathrm{pi}}=\mathrm{Z}_{\mathrm{li}}=\mathrm{Z}_{\mathrm{i}}$ и

$$
\begin{gathered}
\mathrm{B}_{\mathrm{zi}}\left(\mathrm{x}_{\mathrm{k}}, \mathrm{z}_{\mathrm{k}}, \mathrm{x}_{\mathrm{li}}, \mathrm{x}_{\mathrm{pi}}, \mathrm{z}_{\mathrm{i}}, \varphi\right)=\frac{\sin ^{2} \varphi}{2 \pi}\left(\operatorname{arctg} \frac{\mathrm{x}_{\mathrm{k}}-\mathrm{x}_{\mathrm{li}}}{\mathrm{z}_{\mathrm{k}}-\mathrm{z}_{\mathrm{i}}}-\operatorname{arctg} \frac{\mathrm{x}_{\mathrm{k}}-\mathrm{x}_{\mathrm{pi}}}{\mathrm{z}_{\mathrm{k}}-\mathrm{z}_{\mathrm{i}}}\right)+ \\
+\frac{\sin 2 \varphi}{8 \pi} \ln \frac{\left(\mathrm{z}_{\mathrm{k}}-\mathrm{z}_{\mathrm{i}}\right)^{2}+\left(\mathrm{x}_{\mathrm{k}}-\mathrm{x}_{\mathrm{li}}\right)^{2}}{\left(\mathrm{z}_{\mathrm{k}}-\mathrm{z}_{\mathrm{i}}\right)^{2}+\left(\mathrm{x}_{\mathrm{k}}-\mathrm{x}_{\mathrm{pi}}\right)^{2}}
\end{gathered}
$$

Если петля сравнима по размерам с мощностью пачки пластов, а также, если требуется более высокая точность определения их магнитной восприимчивости, вычисление теоретических аномалий $\mathrm{B}_{\mathrm{zi}}$ от каждого пласта в точке $\left(\mathrm{x}_{\mathrm{k}}, \mathrm{z}_{\mathrm{k}}\right)$ может вестись по формулам, учитывающим конкретное намагничение пластов в поле петли сложной формы, то есть путем решения прямой трехмерной задачи по алгоритмам, описанным в главе 2, но без учета размагничивания. Такой подход резко увеличивает время вычислений, и применение его оправдано лишь в некоторых особо важных случаях при детальной разведке месторождений. На более ранних стадиях, как показывает опыт интерпретации в разных рудных провинциях, вполне достаточно вычислять $\mathrm{B}_{\mathrm{zi}}$ по формулам (22.2) или (22.4).

Для поиска минимума функции $(\mathrm{P}+1)$-ой переменной $\Phi$ можно воспользоваться стандартными методами оптимизации, но из-за нелинейности задачи это приведет к большим затратам машинного времени. Значительно эффективнее, осуществляя направленный перебор по нелинейно входящему в $\Phi$ параметру $\varphi$, при каждом его значении решать линейную задачу относительно кажущихся магнитных восприимчивостей пластов. При этом естественно требовать, чтобы задача поиска минимума решалась с заданной точностью при минимальном числе проб значений угла $\varphi$. Можно доказать, что оптимальная стратегия поиска угла $\varphi$ связана с числами Фибоначчи и называется стратегией Фибоначчи [39]. Все другие возможные стратегии могут достигать заданной точности лишь при большем числе проб.

Числа Фибоначчи определяются рекуррентным соотношением:

$$
\mathrm{F}_{\mathrm{n}+2}=\mathrm{F}_{\mathrm{n}+1}+\mathrm{F}_{\mathrm{n}} \quad(\mathrm{n}=1,2, \ldots) ; \mathrm{F}_{1}=\mathrm{F}_{2}=1 \text {. }
$$

Определим вначале число проб, за которое можно найти минимум Ф с заданной точностью определения угла $0<\varphi<180^{\circ}$, равной $\Delta \varphi$. Для этого надо найти минимальное из чисел, удовлетворяющих неравенствам:

или

$$
\frac{\pi}{\mathrm{F}_{\mathrm{n}+2}} \leq \Delta \varphi<\frac{\pi}{\mathrm{F}_{\mathrm{n}+1}}
$$

$$
\mathrm{F}_{\mathrm{n}+1}<\frac{\pi}{\Delta \varphi} \leq \mathrm{F}_{\mathrm{n}+2}
$$


Пусть, например, необходимая точность определения угла $\Delta \varphi$ составляет $0,5^{\circ}$, то есть $\pi / \Delta \varphi=360$. Рассмотрим несколько первых чисел Фибоначчи: $1,1,2,3,5,8,13,21,34,55,89,144$, 233,377 . Анализируя приведенные неравенства, легко убедиться, что минимальное число проб, за которые угол $\varphi$ определяется с точностью $0,5^{\circ}$, равно 12.

Поиск минимума Ф на отрезке от $\varphi_{\text {н }}$ (начальное) по $\varphi_{к}$ (конечное) по стратегии Фибоначчи n-го порядка начинается с выбора двух точек:

$$
\begin{gathered}
\varphi_{1}=\varphi_{\mathrm{H}}+\frac{F_{\mathrm{n}}}{F_{\mathrm{n}+2}}\left(\varphi_{\mathrm{\kappa}}-\varphi_{\mathrm{H}}\right), \\
\varphi_{2}=\varphi_{\mathrm{H}}+\frac{\mathrm{F}_{\mathrm{n}+1}}{\mathrm{~F}_{\mathrm{n}+2}}\left(\varphi_{\mathrm{\kappa}}-\varphi_{\mathrm{H}}\right)=\varphi_{\mathrm{H}}+\varphi_{\mathrm{\kappa}}-\varphi_{1},
\end{gathered}
$$

расположенных на отрезке [ $\left.\varphi_{\mathrm{H}}, \varphi_{\mathrm{K}}\right]$ симметрично, и вычисления $\Phi\left(\varphi_{1}\right)$ и $\Phi\left(\varphi_{2}\right)$. В нашем случае для стратегии 12-го порядка $\varphi_{1}=144 \pi / 377$ и $\varphi_{2}=233 \pi / 377$. Если $\Phi\left(\varphi_{1}\right) \leq \Phi\left(\varphi_{2}\right)$, то для дальнейшего поиска полагаем

$$
\varphi_{\mathrm{H}}^{\prime}=\varphi_{\mathrm{H}}, \quad \varphi_{\mathrm{K}}^{\prime}=\varphi_{2}, \quad \varphi_{2}^{\prime}=\varphi_{1}, \quad \varphi_{1}^{\prime}=\varphi_{\mathrm{H}}^{\prime}+\varphi_{\mathrm{K}}^{\prime}-\varphi_{2}^{\prime}=\varphi_{\mathrm{H}}+\varphi_{2}-\varphi_{1}
$$

и вычисляем $\Phi\left(\varphi_{1}^{\prime}\right)$ и $\Phi\left(\varphi_{2}^{\prime}\right)$. Если же $\Phi\left(\varphi_{1}\right)>\Phi\left(\varphi_{2}\right)$, полагаем

$$
\varphi_{\mathrm{H}}^{\prime}=\varphi_{1}, \quad \varphi_{\mathrm{K}}^{\prime}=\varphi_{\mathrm{K}}, \quad \varphi_{1}^{\prime}=\varphi_{2}, \quad \varphi_{2}^{\prime}=\varphi_{\mathrm{H}}^{\prime}+\varphi_{\mathrm{K}}^{\prime}-\varphi_{1}^{\prime}=\varphi_{1}+\varphi_{\mathrm{K}}-\varphi_{2}
$$

и вычисляем $\Phi\left(\varphi_{1}^{\prime}\right)$ и $\Phi\left(\varphi_{2}^{\prime}\right)$. После каждого этапа точки $\varphi_{1}$ и $\varphi_{2}$ сближаются, а на n-ом этапе (в примере на 12-ом) они совпадут. Вычисленное при этом угле значение Ф характеризует качество подбора, а именно, зная $Ф$, можно рассчитать среднеквадратическую погрешность подбора по формуле

$$
\varepsilon_{\text {под }}=\sqrt{\frac{\Phi}{\mathrm{N}}} .
$$

Минимизация функции Ф с помощью стратегии Фибоначчи сводится к решению $\mathrm{n}$ раз линейной задачи для кажущихся магнитных восприимчивостей $\kappa_{\mathrm{i}}^{\prime}$. При фиксированном угле $\varphi$ определение $\kappa_{\mathrm{i}}^{\prime}$ осуществляется стандартным путем [Приложение].

Таким образом, на втором этапе комплексной количественной интерпретации по данным метода незаземленной петли определены угол падения пачки и кажущиеся магнитные восприимчивости пластов. Для перехода к истинным значениям их необходимо исправить за неоднородность поля петли и за размагничивание. Эти поправки вводятся следующие образом. Вначале вводят поправку за неоднородность поля петли путем умножения кажущихся значений на поправочный коэффициент, зависящий от соотношения параметров пласта и намагничивающей петли. В общем случае этот коэффициент должен определяться с помощью численного моделирования по алгоритмам, приведенным в главе 2, причем, без учета размагничивания. Как правило, исследователь, проводя полевые работы в конкретном регионе, уже представляет интервал глубин залегания пластов и их вероятную мощность. Зная форму и размеры применяемых петель, несложно рассчитать для конкретного региона набор таких поправочных коэффициентов. Во многих регионах верхние кромки пластов располагаются на неболыших глубинах, не превышающих 10-20 м. В этом случае при использовании петель размером $1 \times 1$ км и более поправочный коэффициент, как показывает расчет, весьма близок к единице и составляет примерно 1,02.

Поправку за размагничивание удобно вводить с помощью палетки, построенной по результатам численного моделирования и изображенной на рис. 65. Поскольку глубина $h$ и полумощность пласта b уже известны, с помощью данной палетки уточняется значение магнитной восприимчивости каждого из пластов. На этом завершается второй этап интерпретации. 


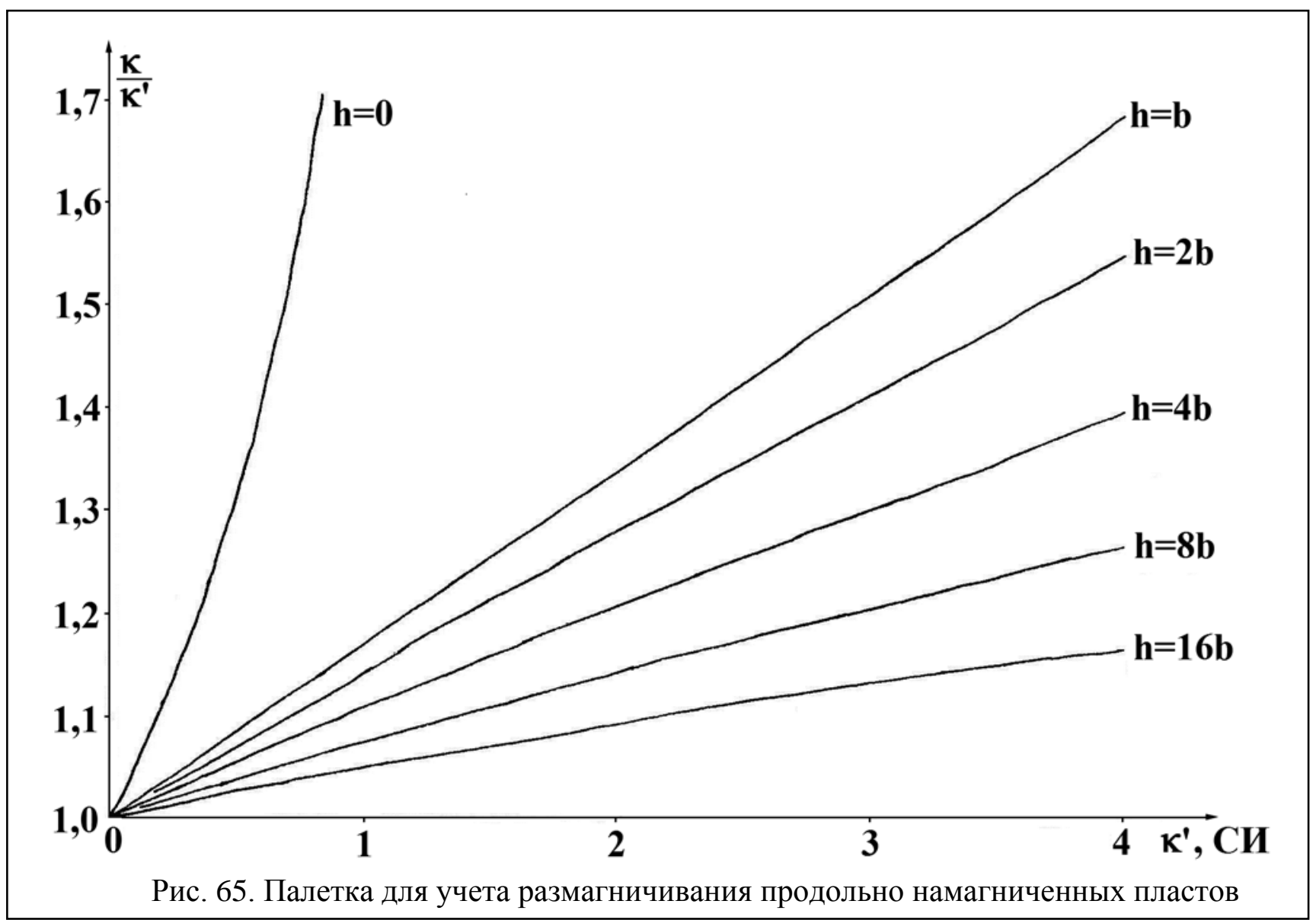

Третий этап заключается в определении по данным магниторазведки проекции вектора естественной остаточной намагниченности каждого из пластов, входящих в пачку, на вертикальную плоскость, проходящую через интерпретационный профиль. Если профиль имеет достаточную длину, то при интерпретации можно оценить глубину до нижней кромки пачки. Достаточной при этом можно считать длину профиля, в несколько раз превышающую глубину нижней кромки. Чаще же интерпретационные профили имеют недостаточную длину, тогда при определении компонент вектора остаточной намагниченности приходится использовать модель неограниченного пласта. При этом компоненты вектора кажущейся остаточной намагниченности $\mathrm{I}_{\text {tani }}$ (продольная) и $\mathrm{I}_{\text {nori }}$ (поперечная) для і-го пласта в соответствии с методом квазирешений определяется путем минимизации квадрата невязки в евклидовом пространстве, имеющей в данном случае следующий вид:

$$
\begin{gathered}
\Phi\left(\mathrm{I}_{\text {tan } 1}^{\prime}, \mathrm{I}_{\text {nor } 1}^{\prime}, \mathrm{I}_{\text {tan } 2}^{\prime}, \mathrm{I}_{\text {nor } 2}^{\prime}, \ldots, \mathrm{I}_{\text {tan } \mathrm{P}}^{\prime}, \mathrm{I}_{\text {norP }}^{\prime}\right)=\sum_{\mathrm{k}=1}^{\mathrm{N}}\left[\mathrm{Z}_{\mathrm{n}}\left(\mathrm{x}_{\mathrm{k}}, \mathrm{z}_{\mathrm{k}}\right)-\sum_{\mathrm{i}=1}^{\mathrm{P}} \mathrm{I}_{\operatorname{tani}}^{\prime} \mathrm{B}_{\text {tani }}\left(\mathrm{x}_{\mathrm{k}}, \mathrm{z}_{\mathrm{k}}, \mathrm{x}_{\mathrm{li}}, \mathrm{z}_{\mathrm{li}}, \mathrm{x}_{\mathrm{pi}}, \mathrm{z}_{\mathrm{pi}}, \varphi\right)-\right. \\
\left.-\sum_{\mathrm{i}=1}^{\mathrm{P}} \mathrm{I}_{\text {tani }}^{\prime} \mathrm{B}_{\text {tani }}\left(\mathrm{x}_{\mathrm{k}}, \mathrm{z}_{\mathrm{k}}, \mathrm{x}_{\mathrm{li}}, \mathrm{z}_{\mathrm{li}}, \mathrm{X}_{\mathrm{pi}}, \mathrm{z}_{\mathrm{pi}}, \varphi\right)-\sum_{\mathrm{i}=1}^{\mathrm{P}} \mathrm{I}_{\text {nori }}^{\prime} \mathrm{B}_{\text {nori }}\left(\mathrm{x}_{\mathrm{k}}, \mathrm{z}_{\mathrm{k}}, \mathrm{x}_{\mathrm{li}}, \mathrm{z}_{\mathrm{li}}, \mathrm{x}_{\mathrm{pi}}, \mathrm{z}_{\mathrm{pi}}, \varphi\right)\right]^{2}=\min , \quad(22.12
\end{gathered}
$$

где $\mathrm{B}_{\text {tani }}$ и $\mathrm{B}_{\text {nori }}$ - соответствующие аномалии от і-го пласта при единичных намагниченностях. Аномалия $Z_{\mathrm{n}}$ в этой формуле представляет собой разность наблюденного поля $\Delta \mathrm{Z}$ и поля $Z_{\mathrm{i}}$, вызванного индуктивной намагниченностью. Поле $Z_{i}$ вычисляется по алгоритмам решения прямой задачи без учета размагничивания от пластов, выделенных на втором этапе, при намагничении их в магнитном поле Земли, известном в районе работ. При этих расчетах используются кажущиеся магнитные восприимчивости пластов, то есть без поправки за размагничивание, но с поправкой за неоднородность поля петли. Такой подход упрощает учет индуктивного намагничения, так как и в поле петли и в субвертикальном земном поле в высоких широтах, влияние размагничивания практически одинаково. 
Вид аномалий $\mathrm{B}_{\text {tani }}$ и $\mathrm{B}_{\text {nori }}$ в формуле (22.12) также может быть различен. Для пласта с наклонной верхней кромкой и неограниченным распространением на глубину:

$$
\begin{aligned}
& \mathrm{B}_{\text {tani }}=\frac{\mu_{0} \sin (\varphi-\alpha)}{2 \pi}\left[\cos \alpha\left(\operatorname{arctg} \frac{\mathrm{x}_{\mathrm{k}}-\mathrm{x}_{\mathrm{li}}}{\mathrm{z}_{\mathrm{k}}-\mathrm{z}_{\mathrm{li}}}-\operatorname{arctg} \frac{\mathrm{x}_{\mathrm{k}}-\mathrm{x}_{\mathrm{pi}}}{\mathrm{z}_{\mathrm{k}}-\mathrm{z}_{\mathrm{pi}}}\right)+\frac{\sin \alpha}{2} \ln \frac{\left(\mathrm{z}_{\mathrm{k}}-\mathrm{z}_{\mathrm{li}}\right)^{2}+\left(\mathrm{x}_{\mathrm{k}}-\mathrm{x}_{\mathrm{li}}\right)^{2}}{\left(\mathrm{z}_{\mathrm{k}}-\mathrm{z}_{\mathrm{pi}}\right)^{2}+\left(\mathrm{x}_{\mathrm{k}}-\mathrm{x}_{\mathrm{pi}}\right)^{2}}\right], \\
& \mathrm{B}_{\text {nori }}=\frac{\mu_{0} \sin (\varphi-\alpha)}{2 \pi}\left[\sin \alpha\left(\operatorname{arctg} \frac{\mathrm{x}_{\mathrm{k}}-\mathrm{x}_{\mathrm{li}}}{\mathrm{z}_{\mathrm{k}}-\mathrm{z}_{\mathrm{li}}}-\operatorname{arctg} \frac{\mathrm{x}_{\mathrm{k}}-\mathrm{x}_{\mathrm{pi}}}{\mathrm{z}_{\mathrm{k}}-\mathrm{z}_{\mathrm{pi}}}\right)+\frac{\cos \alpha}{2} \ln \frac{\left(\mathrm{z}_{\mathrm{k}}-\mathrm{z}_{\mathrm{li}}\right)^{2}+\left(\mathrm{x}_{\mathrm{k}}-\mathrm{x}_{\mathrm{li}}\right)^{2}}{\left(\mathrm{z}_{\mathrm{k}}-\mathrm{z}_{\mathrm{pi}}\right)^{2}+\left(\mathrm{x}_{\mathrm{k}}-\mathrm{x}_{\mathrm{pi}}\right)^{2}}\right],
\end{aligned}
$$

где $\alpha$ определяются по формуле (22.3). При горизонтальном расположении верхней кромки $\alpha=0, \mathrm{z}_{\mathrm{pi}}=\mathrm{Z}_{\mathrm{li}}=\mathrm{Z}_{\mathrm{i}}$, и вид формул упрощается:

$$
\begin{gathered}
\mathrm{B}_{\operatorname{tani}}=\frac{\mu_{0} \sin \varphi}{2 \pi}\left(\operatorname{arctg} \frac{\mathrm{x}_{\mathrm{k}}-\mathrm{x}_{\mathrm{li}}}{\mathrm{z}_{\mathrm{k}}-\mathrm{z}_{\mathrm{li}}}-\operatorname{arctg} \frac{\mathrm{x}_{\mathrm{k}}-\mathrm{x}_{\mathrm{pi}}}{\mathrm{z}_{\mathrm{k}}-\mathrm{z}_{\mathrm{pi}}}\right), \\
\mathrm{B}_{\text {nori }}=\frac{\mu_{0} \sin \varphi}{4 \pi} \ln \frac{\left(\mathrm{z}_{\mathrm{k}}-\mathrm{z}_{\mathrm{li}}\right)^{2}+\left(\mathrm{x}_{\mathrm{k}}-\mathrm{x}_{\mathrm{li}}\right)^{2}}{\left(\mathrm{z}_{\mathrm{k}}-\mathrm{z}_{\mathrm{pi}}\right)^{2}+\left(\mathrm{x}_{\mathrm{k}}-\mathrm{x}_{\mathrm{pi}}\right)^{2}} .
\end{gathered}
$$

Основной интерпретационной процедурой на третьем этапе, понятно, также является решение соответствующей линейной задачи. При ориентировке вектора остаточной намагниченности вертикально вдоль пласта для перехода от кажущейся остаточной намагниченности к истинной можно применять ту же палетку, (рис. 65), так как влияние размагничивания и на первичную индуктивную и на остаточную намагниченность одинаково.

При более сложных условиях влияние размагничивания следует учитывать путем моделирования с помощью алгоритмов, приведенных в главе 2. Необходимо отметить, что для решения большинства геологических задач достаточно информации о кажущейся остаточной намагниченности или даже просто о поле $\mathrm{Z}_{\mathrm{n}}$, созданном остаточной намагниченностью. Если интерпретируемое магнитное поле осложнено региональным фоном, его можно параметризовать, например, в виде полинома невысокой степени и определить при подборе коэффициенты полинома. Такой прием хорош, если при этом не ведется поиск глубины нижней кромки пачки. Если же искать одновременно и параметры фона и глубину нижней кромки, то решение будет крайне неустойчиво из-за коррелированности этих параметров. В этом случае целесообразнее искать положение нижней кромки независимыми способами.

Разработанная методика интерпретации данных комплексной магниторазведки над крутопадающими пластами применялась при изучении железорудных месторождений и рудопроявлений Южной Якутии, КМА, Среднего Приангарья и Казахстана, причем, количество проинтерпретированных с ее помощью профилей измеряется многими сотнями. Рассмотрим некоторые результаты интерпретации по этой методике, полученные при решении типичных геологических задач.

На рис. 66 представлены результаты интерпретации на одном из профилей Тарыннахского месторождения Южной Якутии, которое уже неоднократно упоминалось выше. Это месторождение входит в Ималыкский железорудный район Алданской железорудной провинции. В геологическом строении района преобладают глубокометаморфизованные комплексы архейских кристаллических пород, относящиеся к олекминской и борсалинской сериям. Подчиненное положение занимают осадочные породы среднепротерозойского, верхнепротерозойского и палеозойского возраста. Характерной особенностью Тарыннахского месторождения является неглубокое залегание крутопадающих пластов куммингтонитмагнетитовых, роговообманково-магнетитовых и бессиликатных магнетитовых железистых кварцитов. Руды содержат железа общего 15-60\% (в среднем 29,0 \%), магнетитового железа 20,3-22,6\%, кремнезема 37-60\%, глинозема 0,4-4,23\%, магнезии до 3,98\%, извести до 1,98\%, фосфора 0,01-0,55\% и серы 0,01-0,05\% [219]. В целом они характеризуются как чистые в отношении примесей, бедные, но легкообогатимые. Глубина залегания верхней кромки пачек рудных пластов изменяется в среднем от 4 до 20 м. 

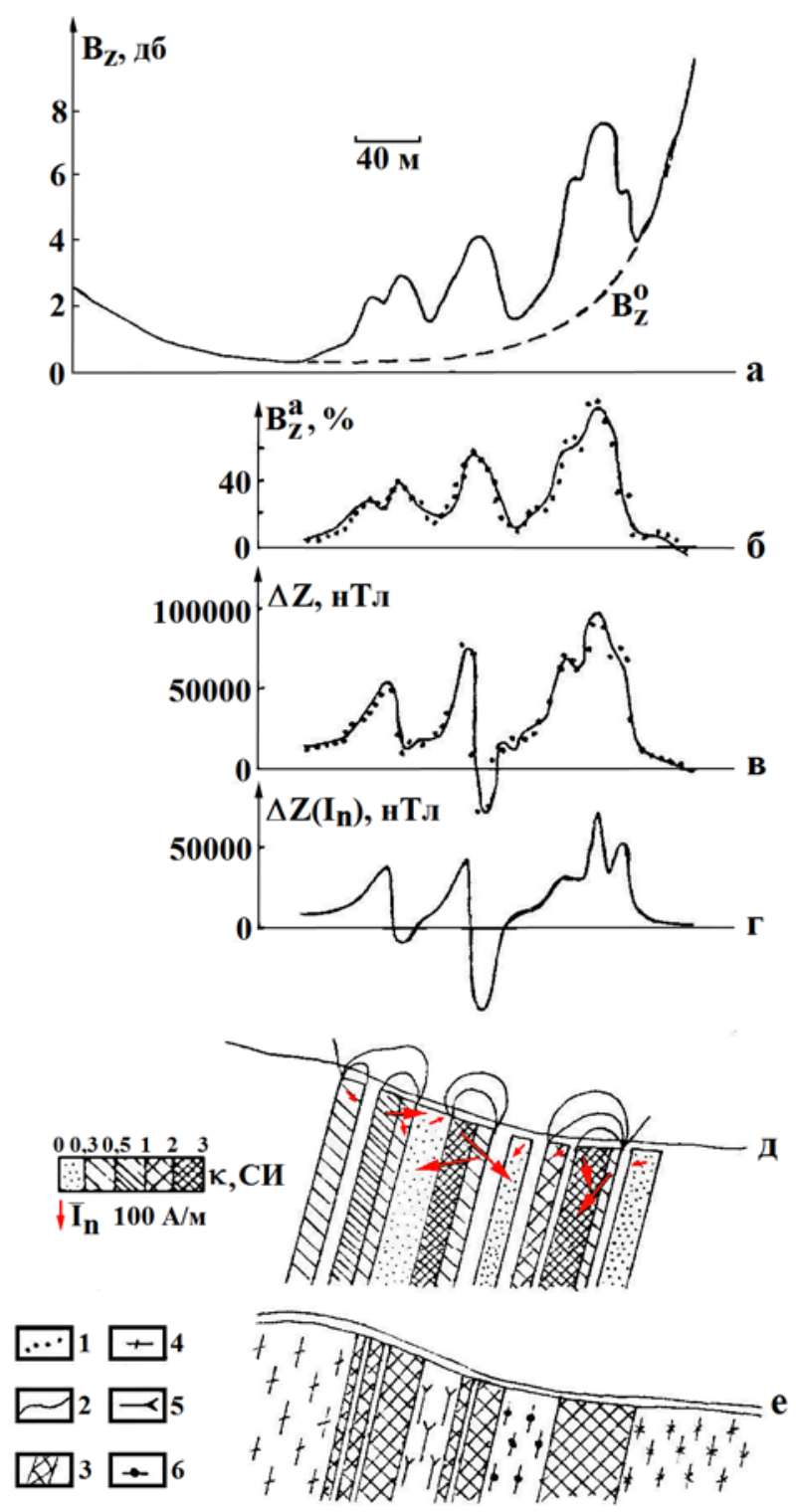

Рис. 66. Результаты комплексной интерпретации данных метода незаземленной петли и магниторазведки на одном из профилей Тарыннахского месторождения Южной Якутии: а) наблюденный график $\mathrm{B}_{\mathrm{z}}$ в петле $500 \times 400$ м на частоте 125 Гц и рассчитанное первичное поле петли $\mathrm{B}_{\mathrm{z}}^{0}$; б) результаты подбора $\mathrm{B}_{\mathrm{z}}^{\mathrm{a}}$; в) результаты подбора $\Delta \mathrm{Z}$; г) расчетный график $\Delta \mathrm{Z}\left(\mathrm{I}_{\mathrm{n}}\right)$; д) изолинии аналитического продолжения $\mathrm{B}_{\mathrm{z}}^{\mathrm{a}}$ и результаты интерпретации; е) схематический геологический разрез (по А.М. Сафонову). 1-наблюденные графики, 2-подобранные графики,

3-железистые кварциты, 4-гранитогнейсы, 5-амфиболовые сланцы, 6-кристаллические сланцы

На графиках $\mathrm{B}_{z}^{\mathrm{a}}$ и $\Delta \mathrm{Z}$ аномалии отдельных пластов проявляются достаточно отчетливо, что и привело к детальному подбору наблюденных аномалий. В результате интерпретации достаточно точно определен угол паления пачки, оценены магнитные свойства входящих в нее пластов и рассчитан график $\Delta \mathrm{Z}\left(\mathrm{I}_{\mathrm{n}}\right)$, характеризующий ту часть аномалии $\Delta \mathrm{Z}$, которая связана с естественной остаточной намагниченностью. Интересно отметить, что на Тарыннахском месторождении верхняя часть пластов, как правило, отличается наличием весьма интенсивной 
остаточной намагниченности, связанной с ударами молний (см. рис. 40) и направленной не только по падению пластов, но и, как видно из рис. 66, почти поперек пласта, причем, изменчивость ее даже внутри единой пачки весьма велика. Такой характер остаточной намагниченности приводит к трудностям при интерпретации аномалий, полученных обычной магниторазведкой, и только комплексная магниторазведка позволила достаточно эффективно изучать месторождения района.

На рис. 67 показаны результаты интерпретации с помощью рассмотренной методики на другом месторождении, относящемся к Ималыкскому железорудному району - Горкитском. На этом месторождении верхняя кромка рудных тел расположена на глубинах свыше 60 м. Естественно, большая глубина залегания верхней кромки не дает возможности детального расчленения пачки, а лишь позволяет определить общую ее конфигурацию. На данном профиле месторождения Горкит установлено, что пачка мощностью 200 м залегает на глубине 70 м. Кажущаяся магнитная восприимчивость пачки составила 0,59 СИ. Истинная средняя магнитная восприимчивость, полученная после введения поправок за неоднородность поля петли и за размагничивание, оказалась равной 0,70 СИ. Векторы индуктивной и остаточной намагниченности, определенные с учетом размагничивания, показаны на рис. 67. Как видно, коэффициент Кёнигсбергера $\mathrm{Q}=\mathrm{I}_{\mathrm{n}} / \mathrm{I}_{\mathrm{i}}$ оказался у руд данного месторождения близким к 0,5 . Результаты бурения подтвердили правильность проведенной интерпретации.

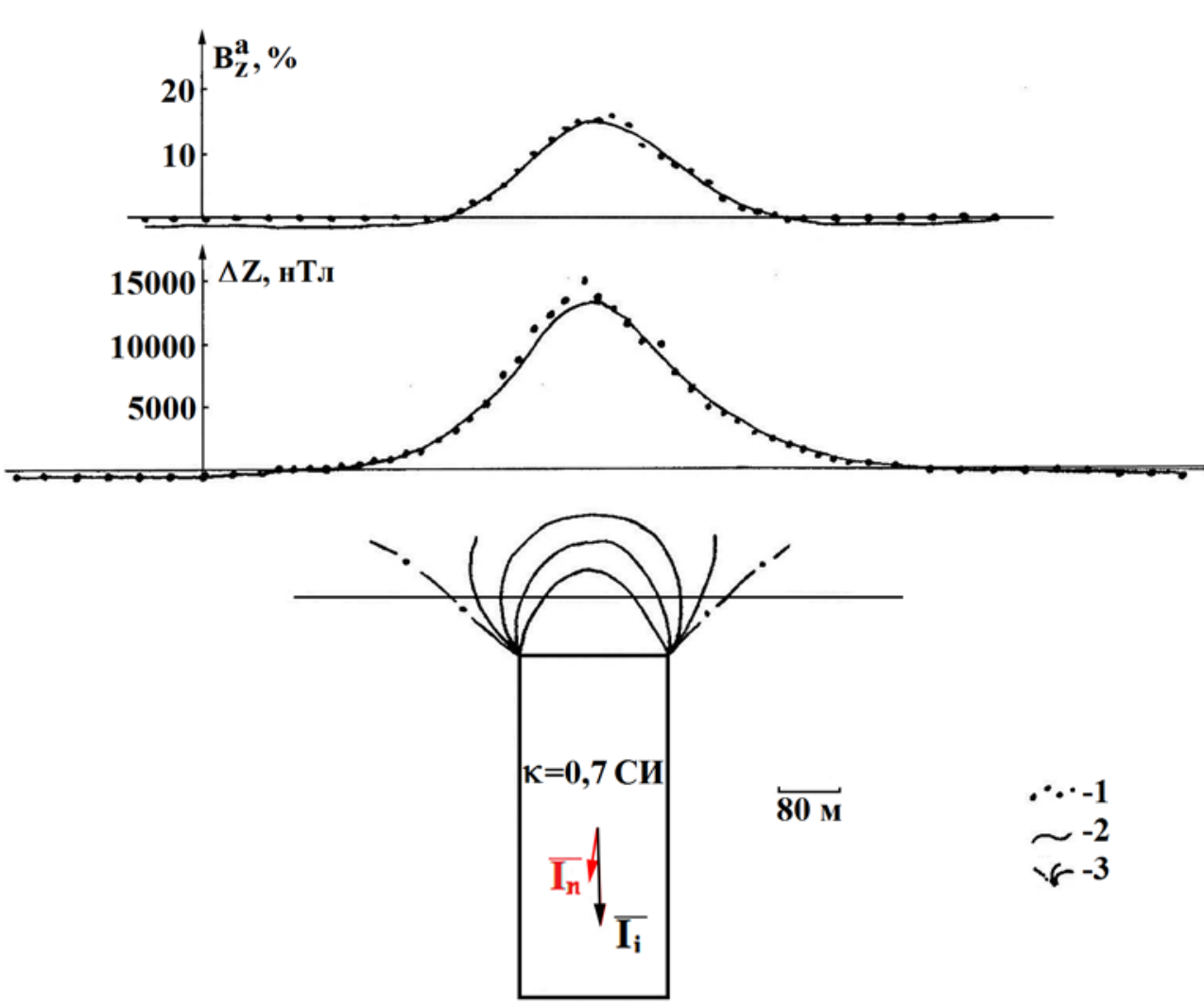

Рис. 67. Результаты комплексной интерпретации данных метода незаземленной петли и магниторазведки на Горкитском месторождении Южной Якутии: 1-наблюденные графики, 2-подобранные графики, 3-изолинии аппроксимационного продолжения $\mathrm{B}_{z}^{\mathrm{a}}$

Следующий пример относится к железорудному месторождению Кумдыколь, расположенному в Центральном Казахстане. Месторождение отмечается в магнитном поле отрицательными магнитными аномалиями с интенсивностью от -15 до -70 мкТл. Результаты горно-буровых работ показали, что аномалии в традиционной магниторазведке совершенно не отражают распределения железных руд в плане, что связано с изменчивостью остаточной намагниченности руд, коэффициент Кёнигсбергера которых колеблется от 0 до 100 [47]. Для 
Ю.И. Блох

Теоретические основы комплексной магниторазведки

выяснения природы магнитных аномалий на месторождении была применена комплексная магниторазведка, включавшая метод незаземленной петли. Работы выполнялись силами Центральной опытно-методической экспедиции под руководством Р.Н. Кириллова при творческом сотрудничестве с МГРИ, и ее основные результаты показаны на рис. 68. Они ярко демонстрируют преимущества применения комплексной магниторазведки.

$\Delta \mathrm{T}$

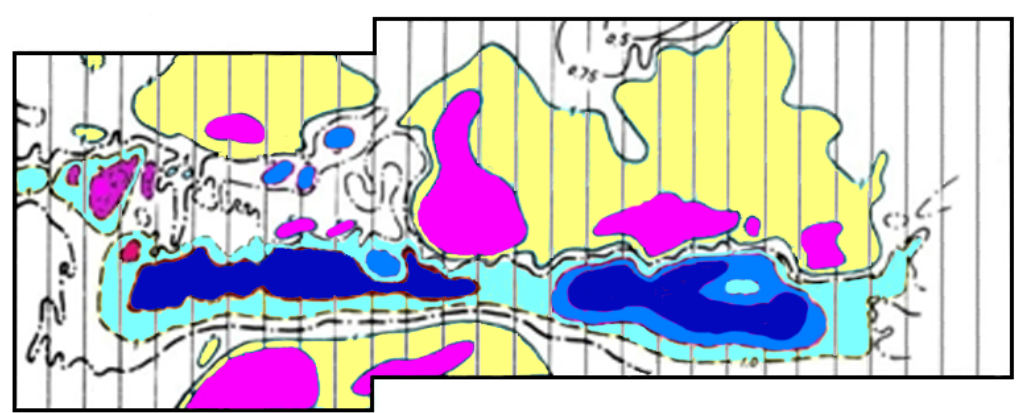

$\Delta \mathbf{Z}$

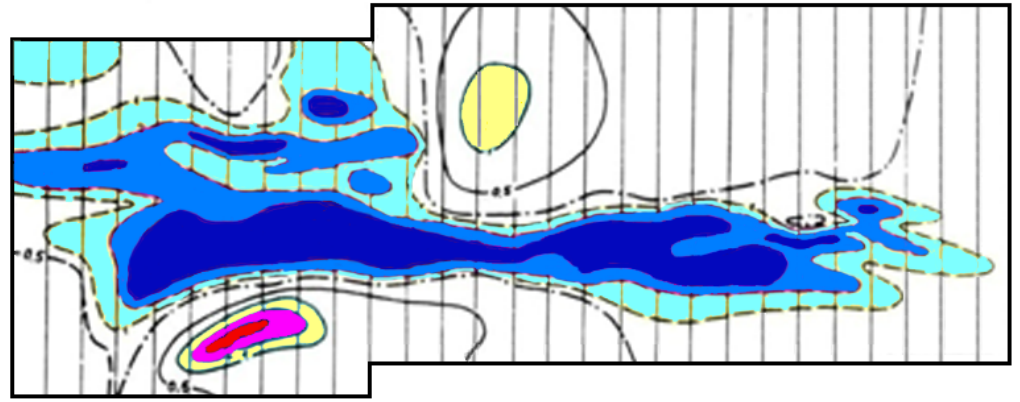

$\max$

$\mathbf{B}_{z}^{\mathbf{a}}$
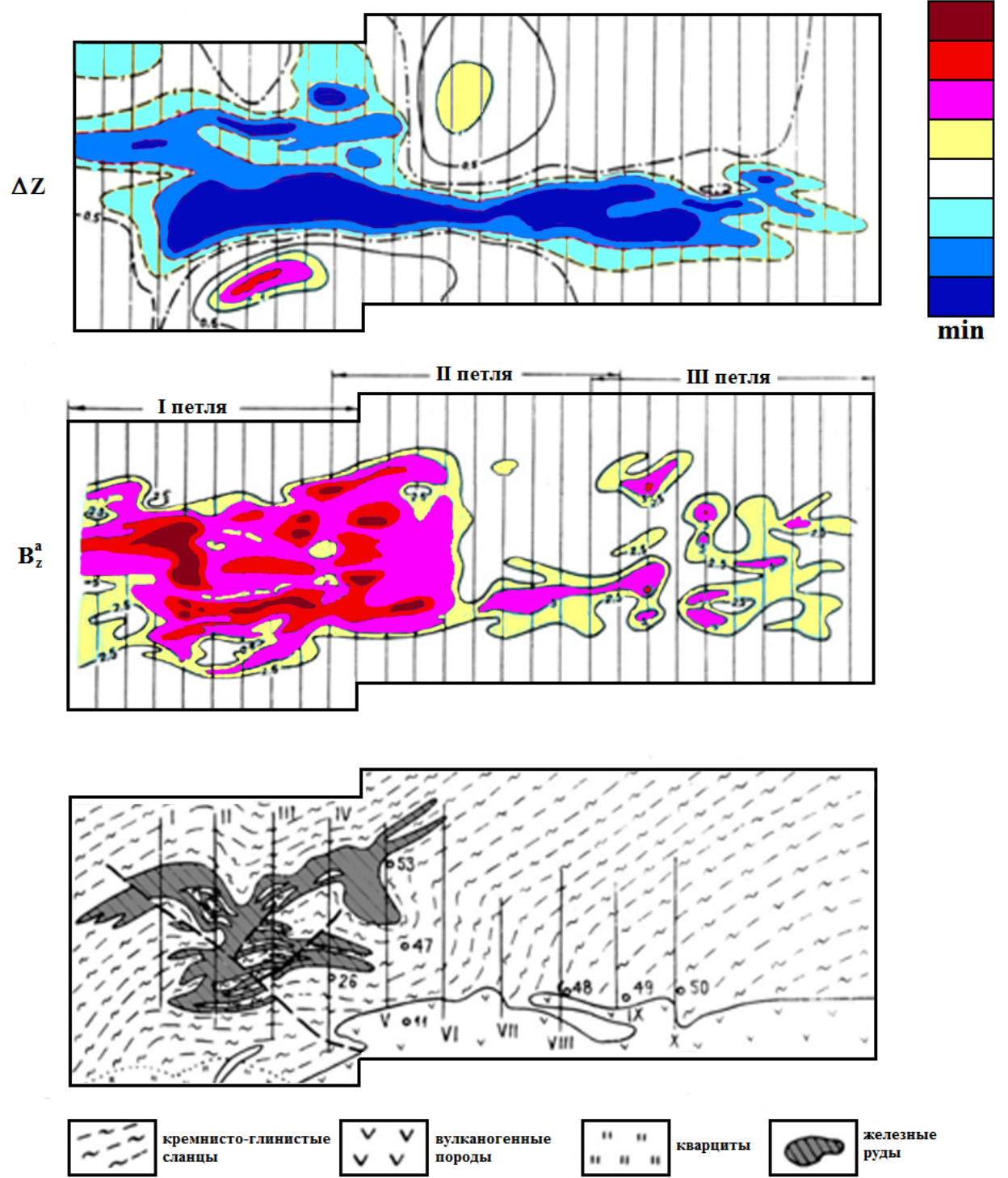

Рис. 68. Результаты комплексной магниторазведки на месторождении Кумдыколь 
Ю.И. Блох

Теоретические основы комплексной магниторазведки

На первой стадии количественной интерпретации все полученные данные были обработаны по рассматриваемой методике. Один из типичных профилей показан на рис. 69, где заметно, что остаточная намагниченность выделенных пластов в целом направлена противоположно индуктивной, причем соотношение между ними меняется при движении с юга на север. Если на южной стороне пачки $\left|\overline{\mathbf{I}}_{\mathrm{n}}\right|>\left|\overline{\mathbf{I}}_{\mathrm{i}}\right|$, то на северной они практически равны, что приводит к уникальной ситуации, когда железные руды с содержанием железа магнетитового до $25 \%$ оказываются имеющими практически нулевую суммарную намагниченность и не создающими заметной магнитной аномалии. В то же время по данным метода незаземленной петли рудные пласты, как видно на рис. 69, выделяются вполне надежно. На второй стадии количественной интерпретации Р.Н. Кирилловым, Т.А. Никитиной и С.К. Утеповым с учетом всей имеющейся информации было проведено моделирование месторождения, что позволило установить строение рудных залежей на глубину до 400 м, а также выделить новые рудные тела в призабойном и межскважинном пространствах [47].

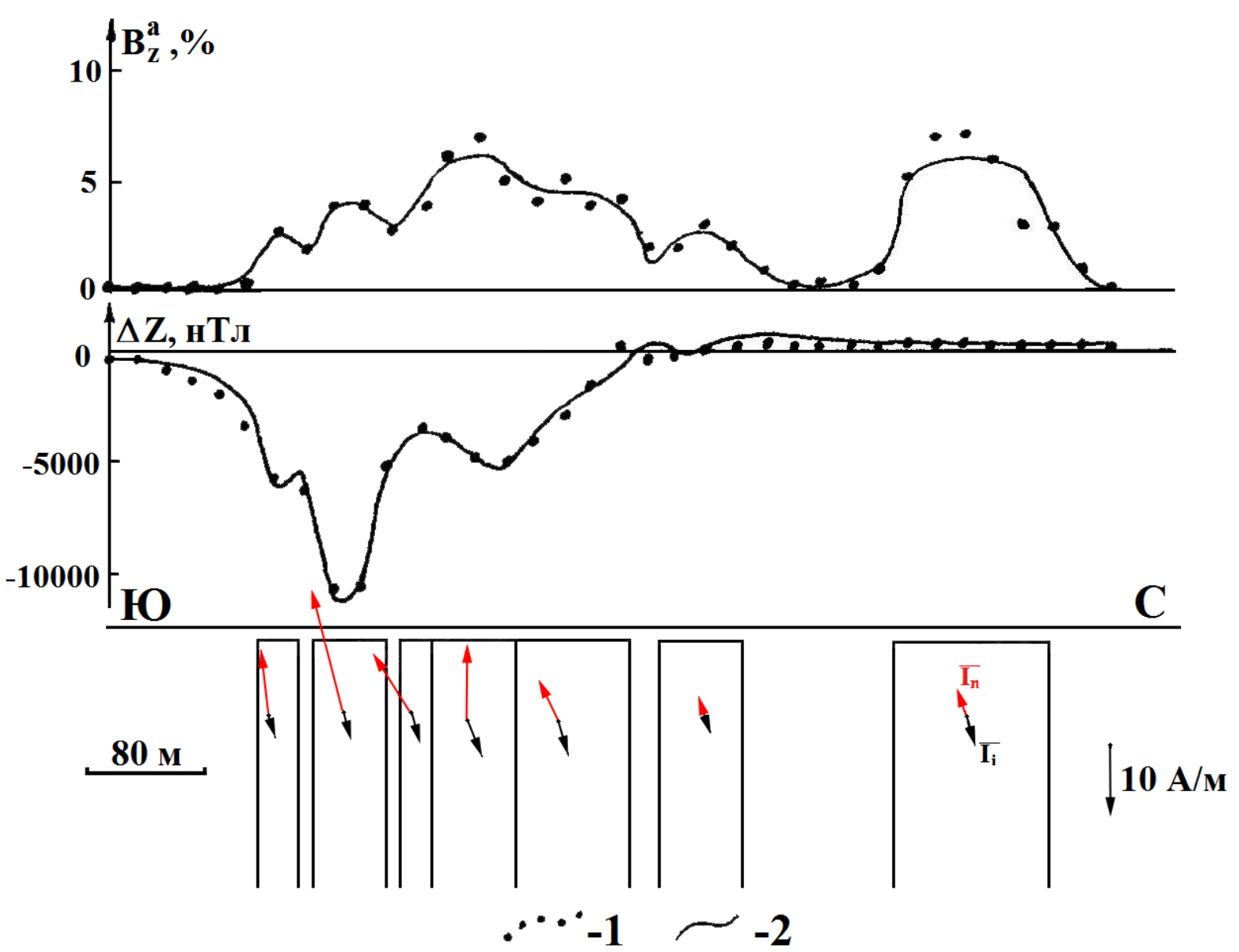

Рис. 69. Результаты комплексной интерпретации данных метода незаземленной петли и магниторазведки на месторождении Кумдыколь в Центральном Казахстане:

1-наблюденные графики, 2-подобранные графики

Предыдущие примеры относились к результатам интерпретации отдельных профилей, но рассматриваемая методика, несмотря на двумерность принимаемой модели пачки, может быть применена и для истолкования результатов площадных съемок. Рассмотрим пример подобной интерпретации по материалам площадных съемок масштаба 1:10000, выполненных при проведении детальных поисков в центральной части Тарыннахского месторождения. На рис. 70 приведен фрагмент карты графиков $\mathrm{B}_{\mathrm{z}}^{\mathrm{a}}$ и $\Delta \mathrm{Z}$ размерами $500 \times 800$ м с вынесенными на него 
Ю.И. Блох

пластами, выделенными в результате интерпретации по каждому из 9 профилей. Для удобства значения $\Delta \mathrm{Z}$ нормированы на значение нормального геомагнитного поля в исследуемом районе и выражены так же, как и $\mathrm{B}_{\mathrm{z}}^{\mathrm{a}}$ в процентах. Магнитная восприимчивость пластов, определенная с учетом поправок за размагничивание и неоднородность намагничивающего поля, показана условной штриховкой. Необходимо ответить, что на рис. 70 пласты показаны генерализованными с учетом полученного качества подбора. Фактически на каждом на профилей выделялось от 11 до 19 пластов, то есть определялось от 78 до 134 параметров (7P+1). Среднеквадратическая погрешность подбора аномалий $\mathrm{B}_{\mathrm{z}}^{\mathrm{a}}=\left(\mathrm{B}_{\mathrm{z}}-\mathrm{B}_{\mathrm{z}}^{0}\right) / \mathrm{B}_{\mathrm{z}}^{0}$ оказалась равной $2,27 \%$, что дало возможность оценить погрешность найденных величин магнитной восприимчивости пластов 0,045 ед. СИ.

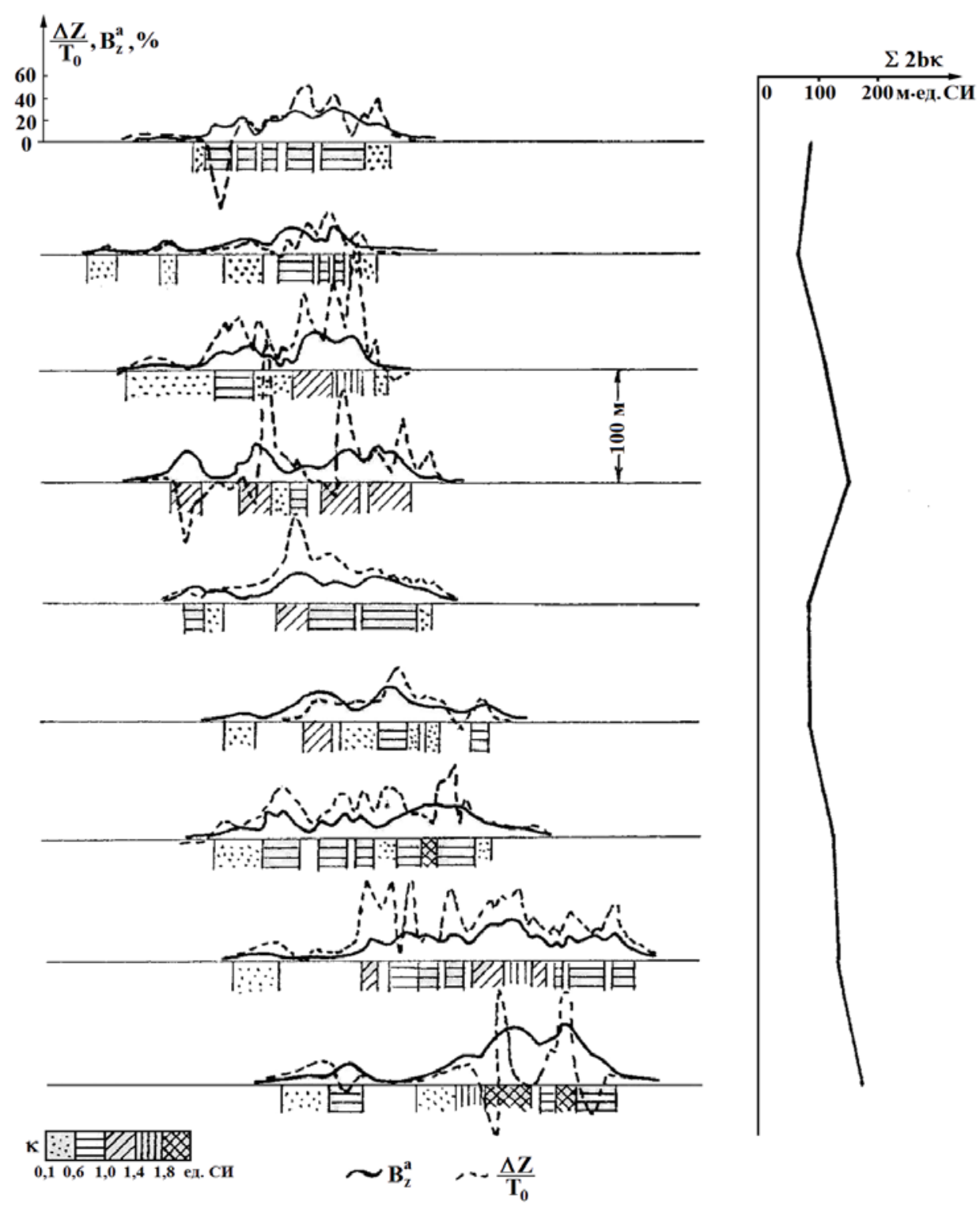

Рис. 70. Фрагмент карты графиков $\mathrm{B}_{\mathrm{z}}^{\mathrm{a}}$ и $\Delta \mathrm{Z}$ Тарыннахского месторождения, полученных при съемке масштаба 1:10000, с обобщенными результатами интерпретации

Сопоставление графиков $\mathrm{B}_{\mathrm{z}}^{\mathrm{a}}$ и $\Delta \mathrm{Z}$ на рис. 70 наглядно демонстрирует изменчивость остаточной намагниченности руд по их простиранию, поэтому при выделении пластов и построении пластовой карты основную роль играют данные метода незаземленной петли. 
Однако роль полученных данных отнюдь не исчерпывается построением пластовой карты. Определенные значения магнитной восприимчивости и видимой мощности пластов могут быть использованы для оценки запасов изучаемого объекта. При этом для каждого выделенного пласта вычисляется произведение видимой мощности $2 \mathrm{~b}$ на магнитную восприимчивость к. Это произведение из-за существования корреляционных связей между к и содержанием железа магнетитового $\mathrm{Fe}_{\text {маг }}$ оказывается аналогичным характеристике метропроцентов железа магнетитового, определяемой по результатам изучения канав, и силу большого объема получаемой информации (в рассматриваемом примере на всех профилях небольшого участка было определено 125 таких произведений) необходимо и целесообразно проводить их статистическую обработку, на рис. 71а представлена гистограмма распределения данного произведения, показывающая, какая часть прогнозных запасов участка относится к каждой из градаций магнитной восприимчивости. Во время проведения детальных поисков на Тарыннахском месторождении была принята следующая корреляционная зависимость между магнитной восприимчивостью в единицах СИ и содержанием железа магнетитового в процентах: $\mathrm{Fe}_{\text {маг }}=2,9+18,9 \kappa$. Наличие корреляционной связи, установленной В.В. Стогнием, позволило переоцифровать гистограмму. В соответствии с ней на рис. 71а проведена вторая ось абсцисс, оцифрованная в процентах содержания магнетитового железа. Таким образом, результаты интерпретации материалов метода незаземленной петли помогают разобраться в структуре запасов месторождения, установить качество преобладающих руд.
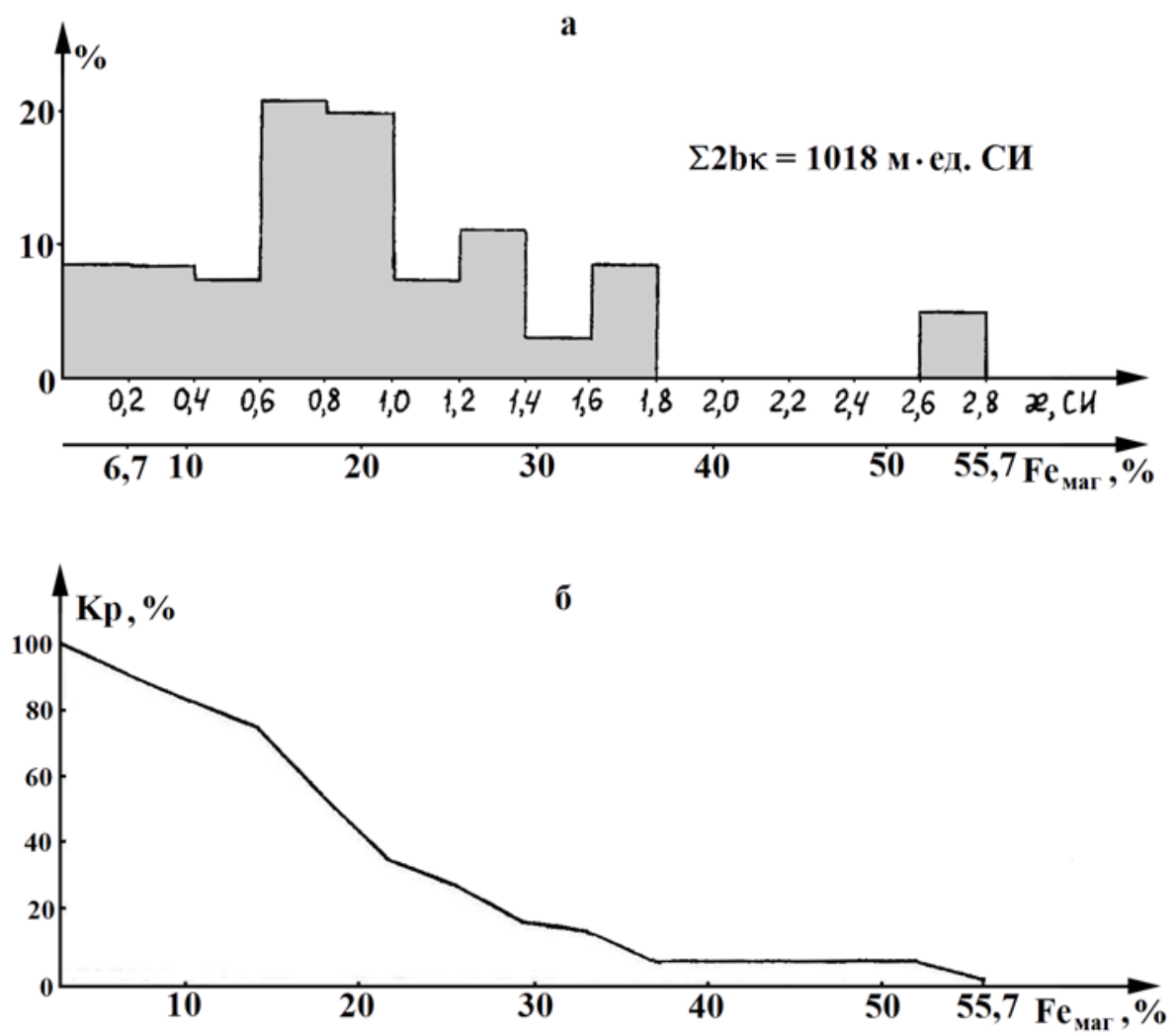

Рис. 71. Результаты статистической обработки материалов, полученных при интерпретации участка, изображенного на рис. 70: а) гистограмма распределения произведения мощности пластов на их магнитную восприимчивость; б) график зависимости коэффициента рудоносности от бортового содержания железа магнетитового

На основании гистограммы, изображенной на рис. 71a, легко построить график зависимости коэффициента рудоносности $\mathrm{K}_{\mathrm{p}}$ от бортового содержания железа магнетитового, показанный на ряс. 71б. С его помощью можно быстро оценить, какая часть запасов участка связана с рудами, содержание железа магнетитового в которых превышает наперед заданную 
величину. Так, при бортовом содержании магнетитового железа 10 \% к кондиционным рудам будет относиться 80 \% запасов участка, а при бортовом содержании $15 \%$ лишь $72 \%$ запасов. Пользуясь принципом аналогии, можно с определенной степенью надежности распространить сведения о коэффициенте рудоносности, полученные на небольшом участке, на все месторождение. Это дает возможность при оценке прогнозных запасов не подбирать аномалии на всех профилях, а пользоваться методами моментов, учитывая ту долю магнитного момента, которая связана с кондиционными рудами, умножением на коэффициент рудоносности. Такой подход значительно упрощает поисковую опенку месторождений. При разведке, естественно, подбор необходимо проводить по всем исследованным профилям.

При изучении рудных тел по простиранию представляет интерес изменение суммы произведений 2 bк для пластов, выделенных на каждом из профилей. На рис. 70 показаны изменения этого параметра от профиля к профилю. Видно, что даже на небольшом участке данная сумма произведений не остается постоянной. В ненарушенных блоках ее изменение монотонно, если же пачка подвергается разрывным нарушениям, в рассматриваемом графике появляются скачки, проявившиеся и на рис. 70. При более детальных съемках местоположение разрывов устанавливается по таким графикам с высокой точностью, дополняя известные способы выявления нарушений.

\section{§ 23. Основы комплексного моделирования магнитных геологических объектов}

Анализ данных наблюдений в комплексной магниторазведке с помощью простых моделей может оказаться достаточным для решения поставленных задач во многих случаях, особенно на ранних стадиях геологоразведочных работ. На поздних же стадиях основную роль, очевидно, играет моделирование геологических объектов.

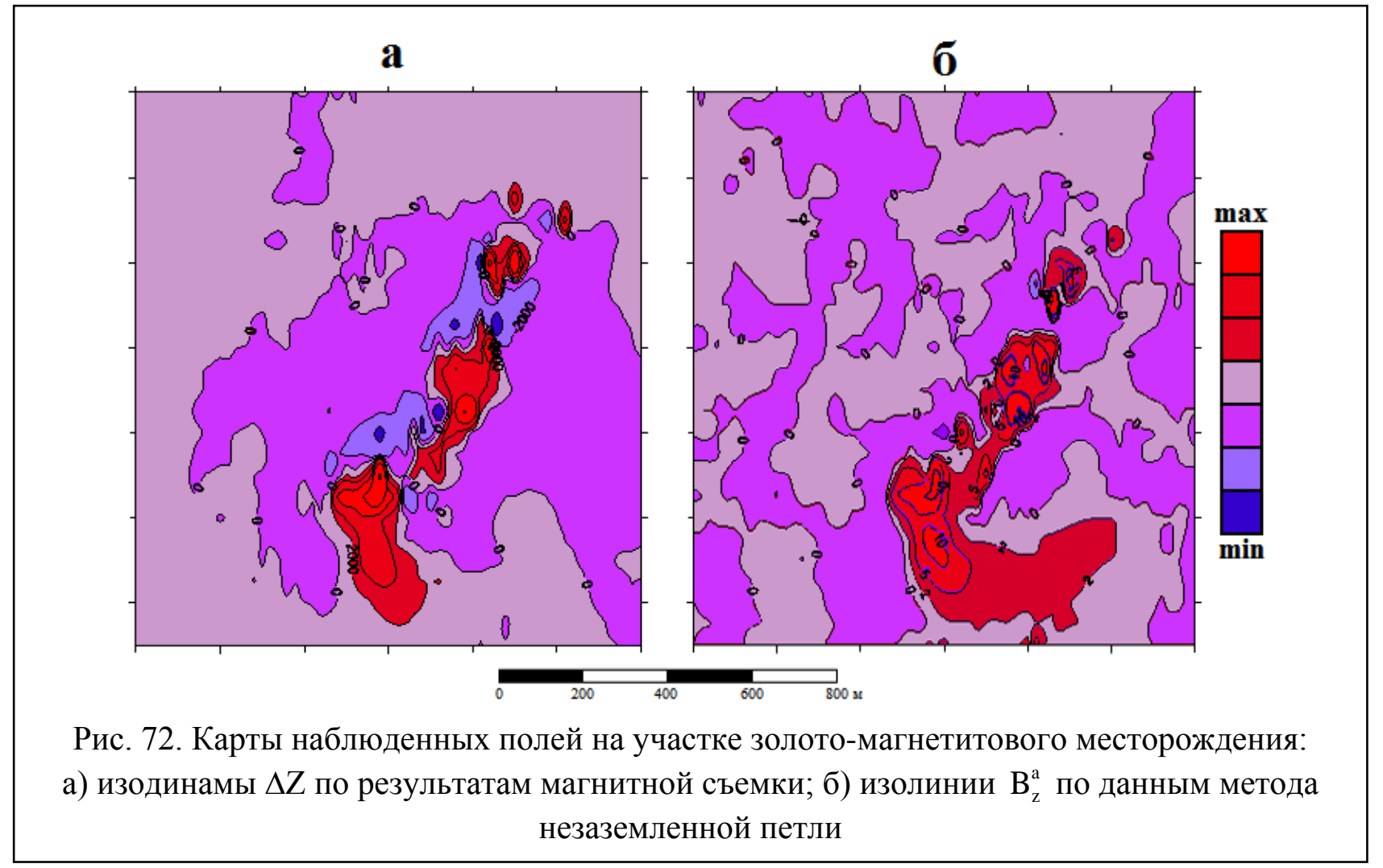

Вообще говоря, компьютерное моделирование аномальных полей при поисках и разведке рудных месторождений стало широко применяться в нашей стране с середины 60-х годов прошлого века. Сама идея моделирования была сформулирована еще в XIX веке, на заре разведочной геофизики, в частности, в работе Ф.А. Слудского 1863 г. [154], однако, ее реальное 
внедрение в геофизическую практику стало возможным только после распространения ЭВМ. Благодаря пионерским работам новосибирских геофизиков Г.Н. Константинова и Л.С. Константиновой, начатым на Таштагольском железорудном месторождении [111], моделирование сильномагнитных геологических объектов по магнитным полям нашло весьма успешное применение в разных железорудных регионах. Опыт моделирования был обобщен группой Г.Н. Константинова в нескольких методических пособиях [112-114] и лег в основу аналогичных работ многих исследователей.

Вообще говоря, в настоящее время построение моделей обычно ведется в одном из трех режимов, различающихся типами применяемых базовых интерпретационных процедур: 1) решение прямой задачи; 2) решение нелинейной обратной задачи и 3 ) комплексное моделирование [39]. Режим решения прямой задачи чаще употребляется на стадии разведки, когда достаточно хорошо известная модель используется преимущественно для проверки качества геологоразведочных работ. Режим решения нелинейной обратной задачи может употребляться, когда интерпретатор имеет достоверные сведения о распределении намагниченности руд и определяет лишь форму сравнительно простой модели. Это крайне редкий случай, и обычно в подобном режиме проводят лишь эквивалентное моделирование с целью выбора одной из нескольких качественно различных гипотез строения изучаемых объектов. Наконец, комплексное моделирование применяется для построения моделей в наиболее сложных случаях, когда отсутствуют достоверные данные об истинном распределении магнитных свойств руд. Естественно, оно применимо не всегда, а только тогда, когда исходных данных достаточно для соблюдения условий единственности решения. Остановимся на этом режиме более подробно.

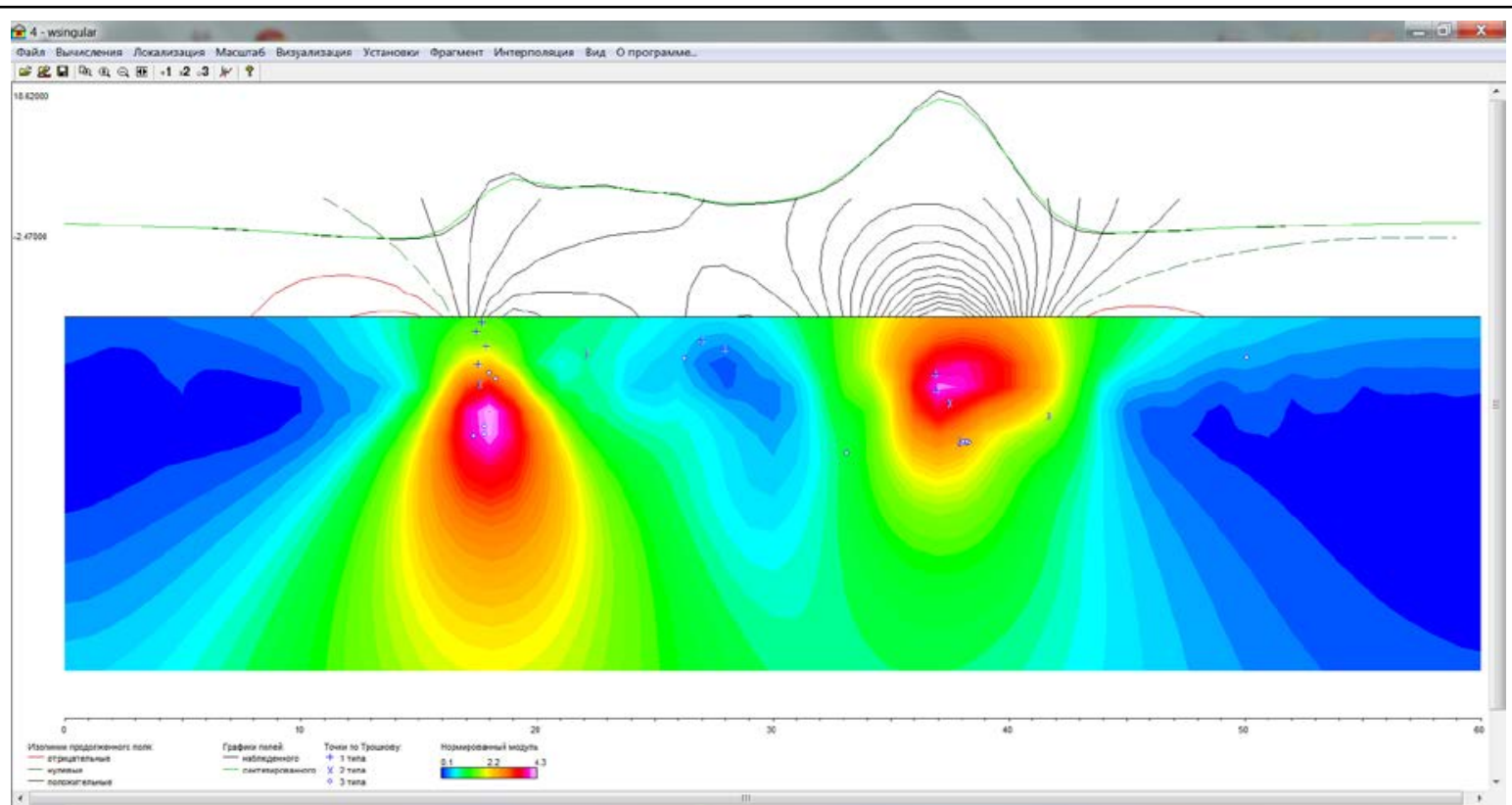

Рис. 73. Сводное изображение, сформированное системой СИНГУЛЯР для локализации особых точек функции, описывающей $\mathrm{B}_{\mathrm{z}}^{\mathrm{a}}$, на одном из профилей золото-магнетитового месторождения

Как правило, моделирование данных комплексной магниторазведки начинается с анализа особых точек функций, описывающих аномальные поля. Проведя локализацию особенностей как по профилям, так и по перпендикулярным им магистралям, интерпретатор получает возможность оконтурить в плане рудные тела и найти глубины их верхних кромок. Затем методами моментов устанавливаются координаты центров масс отдельных тел и их вторые моменты, характеризующие основные элементы залегания. Всей этой информации уже зачастую оказывается достаточно для задания модели, весьма близкой к реальному объекту. 
Ю.И. Блох

Теоретические основы комплексной магниторазведки

Далее подбираются магнитная восприимчивость и остаточная намагниченность руд такие, которые объясняют наблюденные поля. При несовпадении исходных и подбираемых полей модель последовательно корректируется вплоть до удовлетворения всей имеющейся по участку информации. Определенные физические свойства руд можно сопоставить с имеющимися петрофизическими данными и проанализировать их на непротиворечивость. Приведем примеры подобного моделирования.

На рис. 72 показаны карты изодинам $\Delta \mathrm{Z}$ по результатам традиционной магнитной съемки масштаба 1:5000 и изолиний $\mathrm{B}_{\mathrm{z}}^{\mathrm{a}}$ по данным метода незаземленной петли, выполненной в том же масштабе на одном из золото-магнетитовых месторождений Горного Алтая. Магнитное поле изменяется на участке в пределах от -8445 до +54950 нТл, а аномалии $B_{z}^{\mathrm{a}}$ - в пределах от $-11,4$ до $+36,8 \%$ от нормального поля петли. Качественное сопоставление карт показывает практическую идентичность морфологии аномалий, что свидетельствует о преимущественно вертикальном направлении вектора намагниченности руды. Минимумы, обрамляющие максимумы, связанные с рудой, в свою очередь говорят о том, что нижняя кромка рудных тел расположена на небольшой глубине.

Для получения количественных данных на месторождении было проведено комплексное моделирование. Вначале с помощью интегрированной системы «СИНГУЛЯР» на всех профилях были локализованы особые точки функций, описывающих аномальные поля. На рис. 73 представлено сводное изображение, сформированное системой на одном из таких профилей.

Далее аппроксимационным методом были найдены первые гармонические моменты источников полей и вычислены координаты центров масс рудных тел. Затем на нескольких интерпретационных профилях, где измерения проводились с шагом 5 м, были построены двумерные модели рудных тел. На рис. 74 продемонстрирован один из таких профилей. Как показали методы моментов, центр масс рудного тела здесь находится на глубине 42 м. С учетом данных об особых точках и гармонических моментах источников на профилях подобраны разрезы, по которым составлена стартовая трехмерная модель месторождения

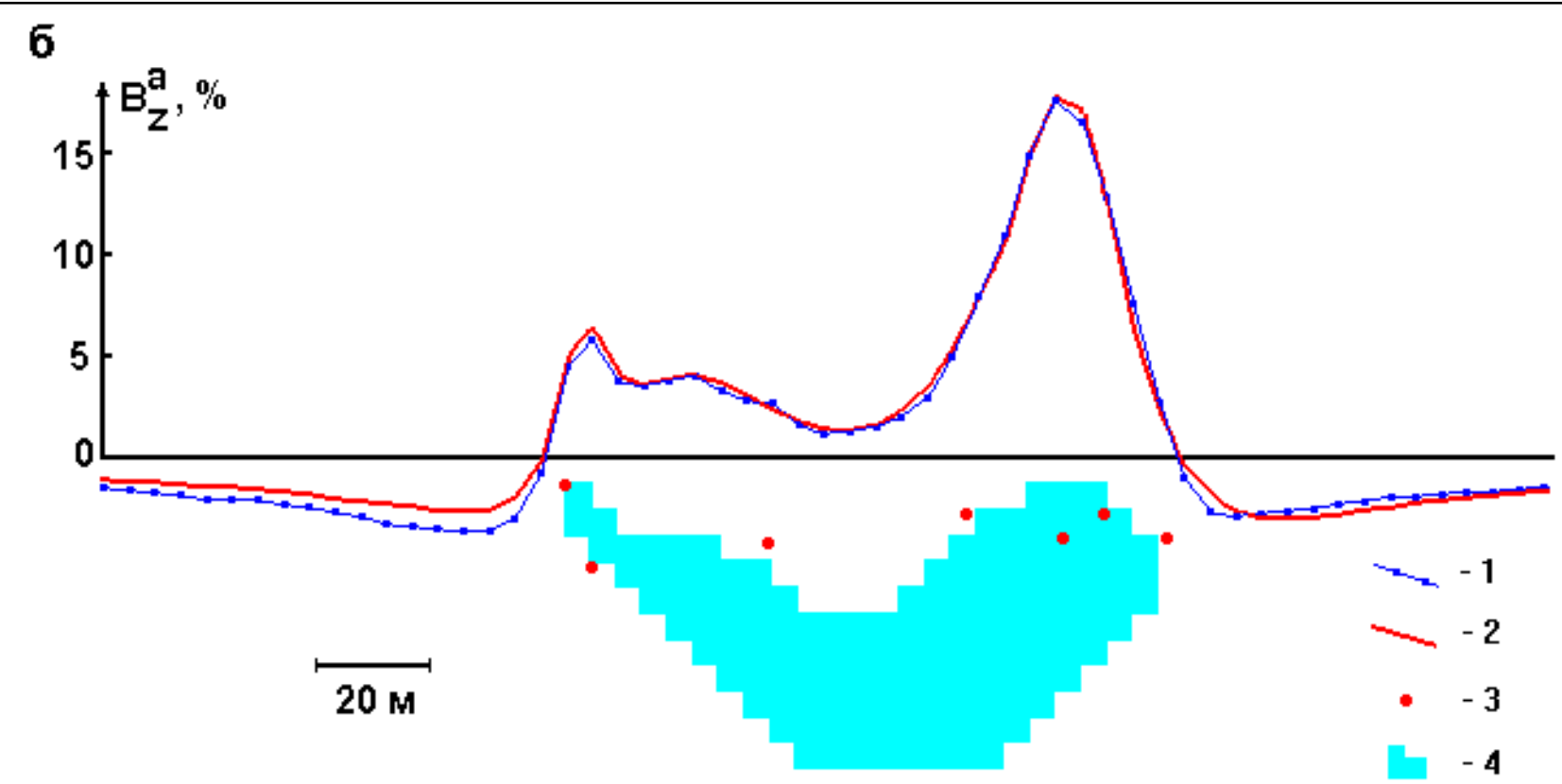

Рис. 74. Интерпретация данных метода незаземленной петли на опорном профиле: 1 - наблюденное поле, 2 - подобранное поле, 3 - расположение локализованных особых

Завершающим этапом явилось построение трехмерной модели рудных тел, показанной на рис. 75 и состоящей из 2135 кубиков с длиной ребра 10 м. На том же рисунке показаны 1586 
точек наблюдения, расположенные на реальной земной поверхности и отражающие достаточно сложный рельеф участка. В процессе оптимизации модели вычисленные от нее поля сравнивались с измеренными в этих точках, а полученные остаточные аномалии использовались для корректировки. В итоге поле $\mathrm{B}_{\mathrm{z}}^{\mathrm{a}}$ было подобрано со среднеквадратической погрешностью $1,51 \%$, близкой к погрешности съемки. Подбор осуществлялся с учетом размагничивания и неоднородности поля намагничивающей петли. Вообще говоря, решив линейную обратную задачу, можно определить кажущуюся магнитную восприимчивость руды, и она оказывается равной $0,636 \pm 0,011$ СИ. На самом деле реальная магнитная восприимчивость

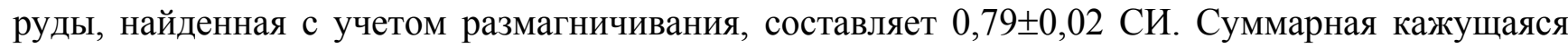
намагниченность руды, определенная по результатам моделирования магнитного поля, оказалась вертикальной и равной $38,3 \pm 1,3$ A/м. Средняя естественная остаточная намагниченность руд после учета размагничивания оказалась равной $10,1 \pm 1,4 \mathrm{~A} / \mathrm{M}$. Вместе с тем, среднеквадратическая погрешность подбора магнитного поля, определенная при однородной остаточной намагниченности руд оказалась равной 2340 нТл. Учет локальных изменений остаточной намагниченности руд позволил подобрать магнитное поле с погрешностью менее 100 нТл. Часть из этих изменений оказалась связанной с последствиями удара молний, но, другая была обязана своим происхождением гидротермальному процессу, в итоге которого магнетитовое месторождение стало золото-магнетитовым. По локальным участкам изменения остаточной намагниченности можно судить о наличии в магнетитовых рудах зон, обогащенных золотом.

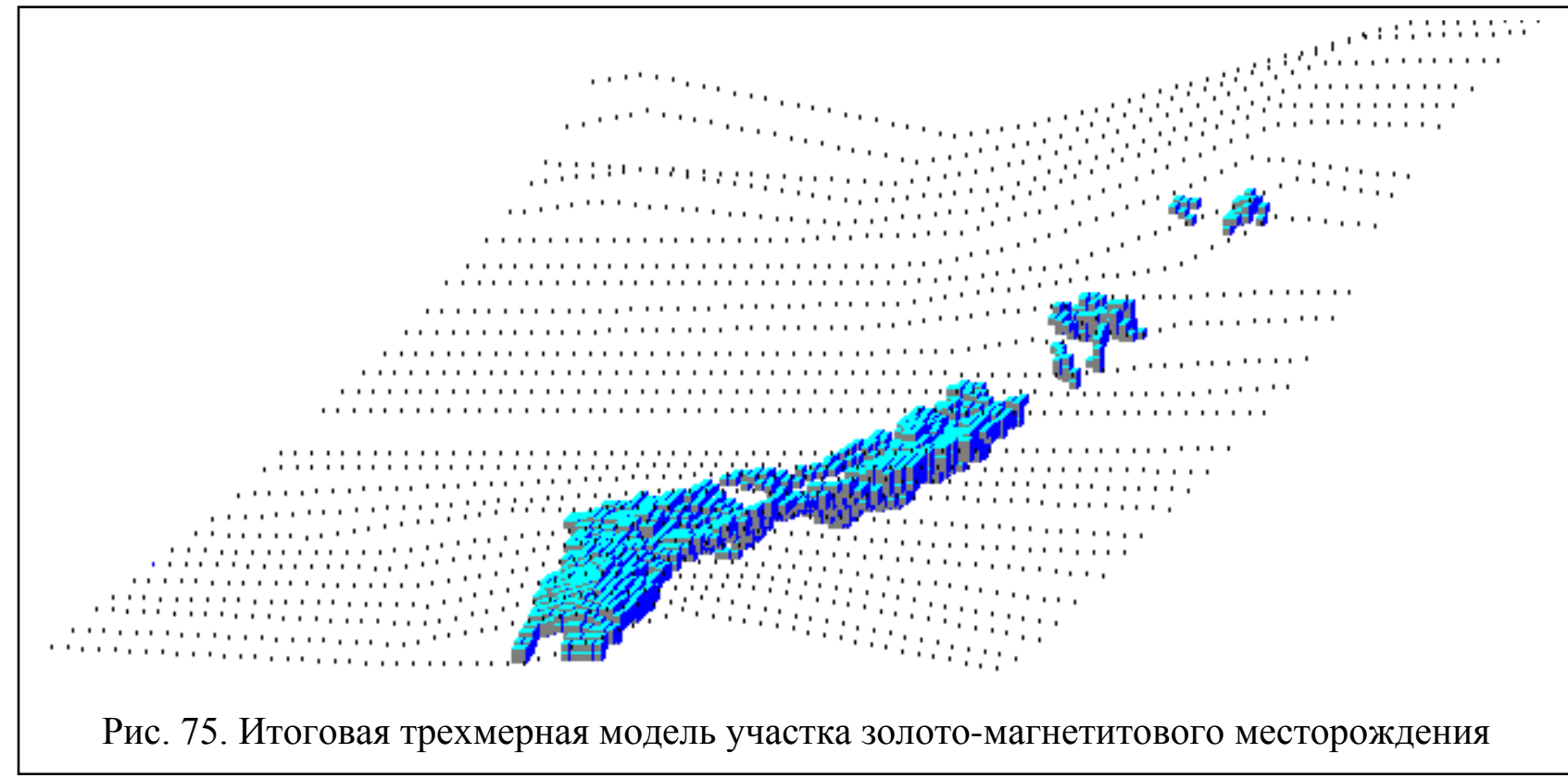

Приведем еще один пример моделирования на участке, оказавшемся безрудным. В одном из районов Горного Алтая была обнаружена магнитная аномалия $\Delta \mathrm{Z}$ амплитудой 12000 нТл (рис. 76а), которая могла оказаться связанной с магнетитовыми рудами. Однако, скважины, пробуренные в эпицентре аномалии, показали лишь наличие гранитоидов. Поскольку глубина скважин была сравнительно небольшой, аномалию предположили вызванной глубокозалегающим железорудным месторождением. Тем не менее, прежде чем бурить глубокие скважины, было решено провести на участке размерами $2 \times 1$ км комплексную магниторазведку с привлечением метода незаземленной петли по сети $100 \times 50$ м, чтобы убедиться в его перспективности. На рис. $76 б$ показаны результаты съемки в виде карты изолиний $\mathrm{B}_{\mathrm{z}}^{\mathrm{a}}$. Даже качественный анализ карты показывает, что аномалия $\mathrm{B}_{\mathrm{z}}^{\mathrm{a}}$, амплитуда которой чуть более 5\% от нормального поля петли, чересчур мала, чтобы быть вызванной 
Ю.И. Блох

Теоретические основы комплексной магниторазведки

железными рудами. При этом морфология аномалии указывает на ее приповерхностные источники, вероятно связанные с исключительно высокой остаточной намагниченностью гранитоидов. Чтобы окончательно убедиться в данной гипотезе, было проведено комплексное моделирование.

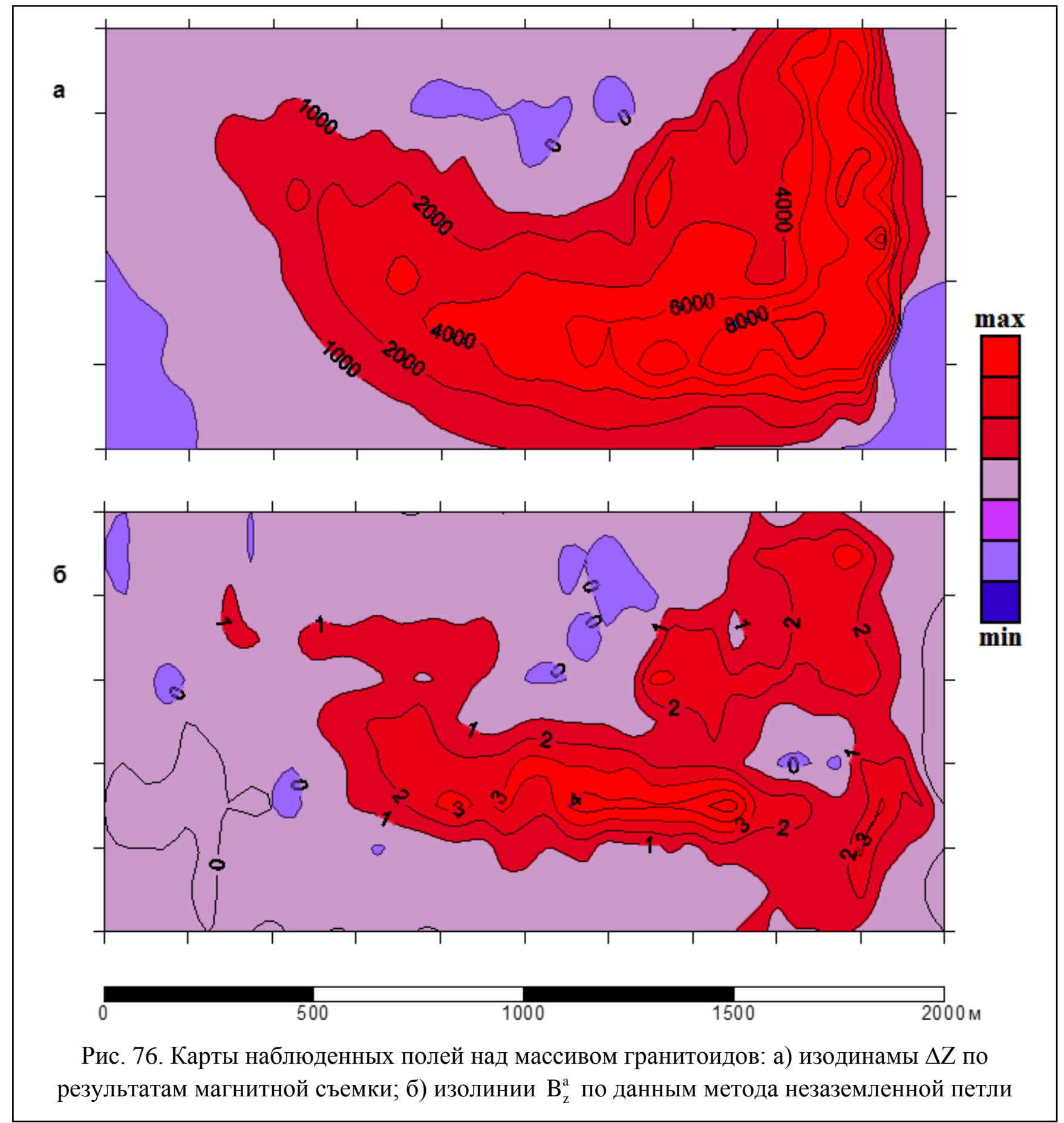

Вначале на основе методов особых точек и моментов с учетом данных бурения была создана стартовая трехмерная модель интрузива в виде многогранника (рис. 77a), но в процессе ее оптимизации выяснилась необходимость выделить внутри нее 4 петромагнитных типа пород. Это гораздо проще делать при аппроксимации объекта набором кубиков, тем более что для моделирования данных требуется учитывать неоднородность поля петли и размагничивание. Оптимизация привела к итоговой модели, представленной на рис. 776 и состоящей из 319 кубиков с длиной ребра 50 м. Среднеквадратические погрешности подбора составили 0,57\% для 
данных метода незаземленной петли и 72 нТл для магнитного поля. В итоге оказалось, что наблюденные поля сформированы гранитоидами, магнитная восприимчивость которых изменяется от 0,019 до 0,097 СИ. Остаточная намагниченность в некоторых разностях в 9,8 раз превышает индуктивную и достигает уникальной для гранитоидов величины 23,4 A/м.

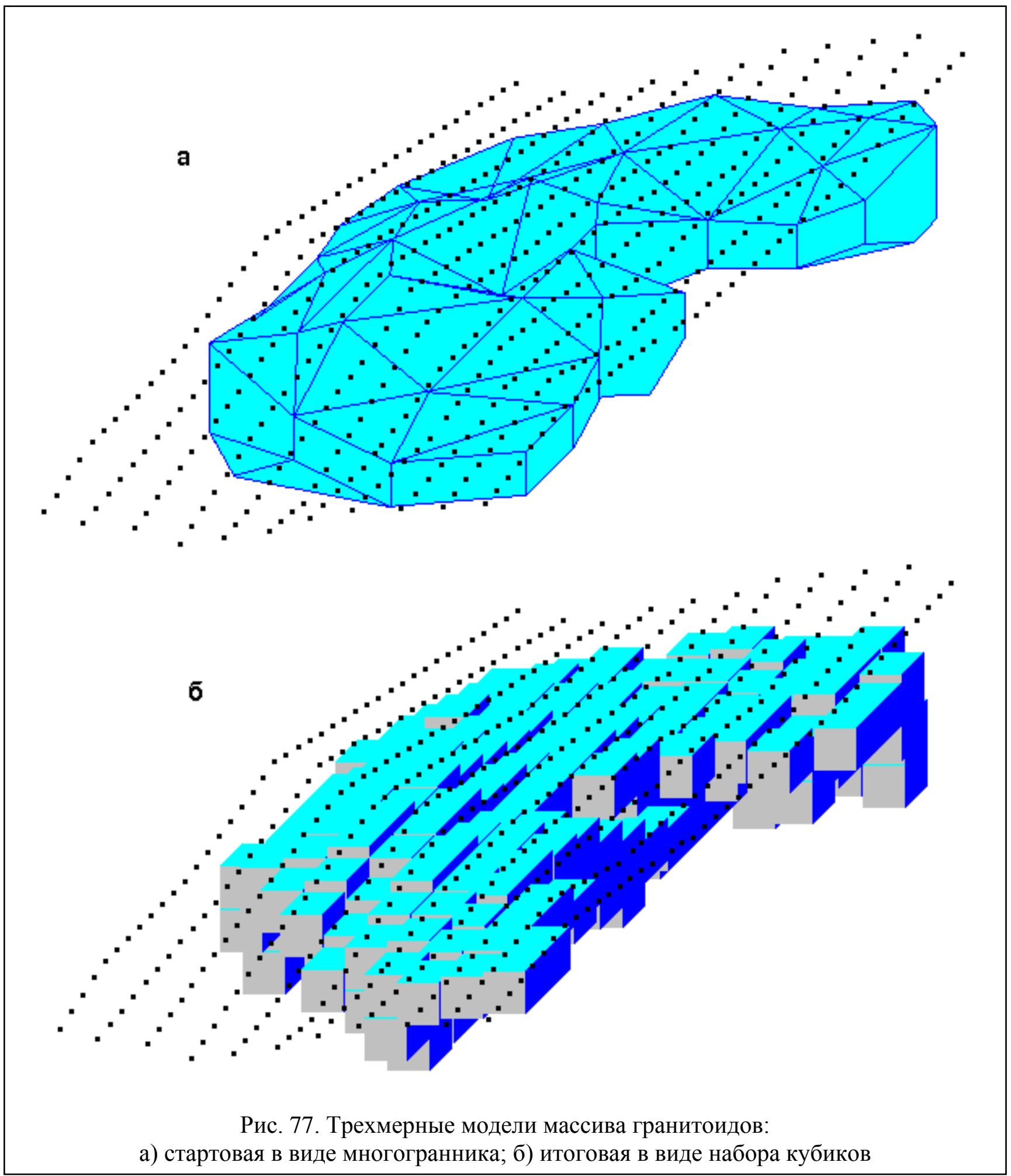

Таким образом, комплексное моделирование сильномагнитных объектов дает возможность успешно решать широкий круг достаточно сложных геологических задач в условиях минимума требуемой априорной информации. 


\section{ЗАКЛЮЧЕНИЕ}

Рассмотренные в данной работе теоретические основы, как надеется автор, позволили внимательному читателю понять, сколь существенно расширяются возможности геофизиков, если они могут применять для решения геологических задач комплексную магниторазведку. Тем не менее, для этого необходимо, как минимум, иметь в распоряжении соответствующую аппаратуру.

Ранее, если не принимать во внимание штучные макеты, большинство производственных съемок НЧИМ выполнялось с помощью аппаратуры типа ИКС, снабженной магнитоиндукционными датчиками МИД [45]. В настоящее время выпускаются современные генераторы и измерители, с помощью которых можно было бы проводить полевые работы на месторождениях сильномагнитных руд с относительной точностью порядка $1 \%$, но магнитоиндукционных датчиков уже практически не осталось. Их необходимо разрабатывать заново на современной технической базе, что, вообще говоря, не является принципиально сложной проблемой.

Более сложна ситуация с аппаратурой для высокоточных измерений, с помощью которой можно изучать среднемагнитные геологические объекты. Измерители ВТИМП-1 для съемок с относительной точностью около 0,1\% [45], имеются всего в нескольких экземплярах. Здесь современный элементный уровень позволяет осуществить качественный скачок и создать существенно более точную и мобильную аппаратуру. Все сказанное в полной мере относится и к аппаратуре МИП.

Традиционно в комплексной магниторазведке изучалась вертикальная компонента аномального поля. Сейчас традиционная магниторазведка обычно реализуется на базе модульных съемок с протонными и квантовыми магнитометрами, и НЧИМ, как и МИП вполне можно развивать в режиме модульной съемки, для чего, конечно, надо создавать новые датчики. Отметим, что в МИП положительный опыт применения модульных магнитометров уже имеется [161, 170].

Для полевых работ методом МВМ крайне важно разработать прецизионные и легкие трехкомпонентные датчики. Особо перспективно здесь применение сверхпроводящих квантовых интерферометров - СКВИДов, которые были опробованы для этих целей еще в 1976-1977 годах [54].

Еще одним важным аспектом успешного применения комплексной магниторазведки является совершенствование методики полевых работ. Ранее точность съемок существенно ограничивалась возможностями их топогеодезического обеспечения, так как при выделении аномалий необходимо учитывать первичное поле источников, для чего надо с высокой точностью знать геометрию источников и положение пунктов измерений. Сейчас для этого можно эффективно применять доступные системы спутниковой навигации: ГЛОНАСС, GPS и др. Обратим внимание на то, что более критичной для вычисления первичного поля является точность определения горизонтальных координат [45], и здесь в полевых условиях у спутниковой навигации фактически нет реальных конкурентов.

Автор полагает очевидным, что уже в недалеком будущем комплексную магниторазведку неминуемо ждут возрождение и расцвет, и он надеется, что его труд по сбору и связыванию ее разорванных нитей окажется полезным геофизическому сообществу. 


\section{ПРИЛОЖЕНИЕ}

\section{Решение линейных обратных задач}

В комплексной магниторазведке почти повсеместно требуется решение линейных задач подбора. Они характеризуются тем, что оператор D решения прямой задачи в них является прямоугольной матрицей $\mathrm{A}$, состоящей из $\mathrm{m}$ строк и $\mathrm{n}$ столбцов. Модель $\overline{\mathrm{p}}$ и исходные данные $\overline{\mathrm{u}}$ при этом понимаются как векторы-столбцы, имеющие соответственно $\mathrm{n}$ и $\mathrm{m}$ элементов [39]. К наиболее типичным среди таких задач относится определение по данным обычной магниторазведки компонент вектора намагниченности объектов, местоположение, форма и магнитная восприимчивость которых известны по данным МВМ, МИП или НЧИМ. Рассмотрим решение такой задачи для уединенного тела в условиях, когда исследуемая магнитная аномалия $\Delta \mathrm{Z}$ осложнена линейным региональным фоном. При этом отметим, что возрастание числа изучаемых тел не выводит задачу за рамки линейных, но лишь увеличивает ее размерность. Конечно, тела должны быть разнесены по горизонтали, иначе возникающие компенсационные эффекты сделают задачу настолько неустойчивой, что решать ее станет возможным (да и то не во всех ситуациях) лишь применяя дополнительные методы регуляризации [39].

Итак, пусть известны значения магнитной аномалии $\Delta \mathrm{Z}$ в m точках на поверхности Земли, при этом, вообще говоря, безразлично, является ли дневная поверхность горизонтальной или имеет сложную форму. Аномалии будем считать осложненными линейным региональным фоном, описываемым функцией $a x+b y+c$ с неизвестными коэффициентами $a, b, c$. На основании априорной информации известен объект, компоненты вектора намагниченности которого $\mathrm{I}_{\mathrm{x}}, \mathrm{I}_{\mathrm{y}}$ и $\mathrm{I}_{\mathrm{z}}$ требуется определить.

Поле интерпретационной модели в k-ой точке с координатами $\left(\mathrm{x}_{\mathrm{k}}, \mathrm{y}_{\mathrm{k}}, \mathrm{z}_{\mathrm{k}}\right)$ с учетом линейного регионального фона может быть представлено в форме

$$
\Delta \mathrm{Z}_{\mathrm{m}}\left(\mathrm{x}_{\mathrm{k}}, \mathrm{y}_{\mathrm{k}}, \mathrm{z}_{\mathrm{k}}\right)=\mathrm{ax}_{\mathrm{k}}+\mathrm{by}_{\mathrm{k}}+\mathrm{c}+\mathrm{I}_{\mathrm{x}} \Gamma_{1}\left(\mathrm{x}_{\mathrm{k}}, \mathrm{y}_{\mathrm{k}}, \mathrm{z}_{\mathrm{k}}\right)+\mathrm{I}_{\mathrm{y}} \Gamma_{2}\left(\mathrm{x}_{\mathrm{k}}, \mathrm{y}_{\mathrm{k}}, \mathrm{z}_{\mathrm{k}}\right)+\mathrm{I}_{\mathrm{z}} \Gamma_{3}\left(\mathrm{x}_{\mathrm{k}}, \mathrm{y}_{\mathrm{k}}, \mathrm{z}_{\mathrm{k}}\right),
$$

где $\Gamma_{1}, \Gamma_{2}, \Gamma_{3}-$ известные значения, характеризующие в k-ой точке величину компоненты $\Delta \mathrm{Z}$ аномального магнитного поля, создаваемой заданным объектом при единичных намагниченностях $\mathrm{I}_{\mathrm{x}}, \mathrm{I}_{\mathrm{y}}$ и $\mathrm{I}_{\mathrm{z}}$ соответственно. Квазирешение данной задачи может быть найдено путем минимизации квадрата невязки решаемого операторного уравнения, записанной в виде

$$
\begin{gathered}
\Phi\left(\mathrm{a}, \mathrm{b}, \mathrm{c}, \mathrm{I}_{\mathrm{x}}, \mathrm{I}_{\mathrm{y}}, \mathrm{I}_{\mathrm{z}}\right)= \\
=\sum_{\mathrm{k}=1}^{\mathrm{m}}\left[\Delta \mathrm{Z}\left(\mathrm{x}_{\mathrm{k}}, \mathrm{y}_{\mathrm{k}}, \mathrm{z}_{\mathrm{k}}\right)-\mathrm{ax}_{\mathrm{k}}-\mathrm{by}_{\mathrm{k}}-\mathrm{c}-\mathrm{I}_{\mathrm{x}} \Gamma_{1}\left(\mathrm{x}_{\mathrm{k}}, \mathrm{y}_{\mathrm{k}}, \mathrm{z}_{\mathrm{k}}\right)-\mathrm{I}_{\mathrm{y}} \Gamma_{2}\left(\mathrm{x}_{\mathrm{k}}, \mathrm{y}_{\mathrm{k}}, \mathrm{z}_{\mathrm{k}}\right)-\mathrm{I}_{\mathrm{z}} \Gamma_{3}\left(\mathrm{x}_{\mathrm{k}}, \mathrm{y}_{\mathrm{k}}, \mathrm{z}_{\mathrm{k}}\right)\right]^{2}= \\
=\text { min. }
\end{gathered}
$$

При этом в силу линейности оператора решения прямой задачи определение искомых параметров в вычислительном плане сводится к решению достаточно проработанных проблем линейной алгебры.

Типичной при решении линейных обратных задач в комплексной магниторазведке является ситуация, когда число точек наблюдения превышает число искомых параметров, в нашем примере $\mathrm{m}>6$. Тогда задача сводится к устойчивому решению переопределенной системы линейных алгебраических уравнений (СЛАУ). Эта система, называемая системой нормальных уравнений, получается из необходимых условий экстремума минимизируемой функции Ф [39]. Опустим для краткости обозначений пределы суммирования, а также аргументы функций, тогда СЛАУ для нашей задачи примет следующий вид: 


$$
\begin{gathered}
\mathrm{a} \sum \mathrm{x}_{\mathrm{k}}^{2}+\mathrm{b} \sum \mathrm{x}_{\mathrm{k}} \mathrm{y}_{\mathrm{k}}+\mathrm{c} \sum \mathrm{x}_{\mathrm{k}}+\mathrm{I}_{\mathrm{x}} \sum \mathrm{x}_{\mathrm{k}} \Gamma_{1}+\mathrm{I}_{\mathrm{y}} \sum \mathrm{x}_{\mathrm{k}} \Gamma_{2}+\mathrm{I}_{\mathrm{z}} \sum \mathrm{x}_{\mathrm{k}} \Gamma_{3}=\sum \mathrm{x}_{\mathrm{k}} \Delta \mathrm{Z}_{\mathrm{k}}, \\
\mathrm{a} \sum \mathrm{y}_{\mathrm{k}} \mathrm{x}_{\mathrm{k}}+\mathrm{b} \sum \mathrm{y}_{\mathrm{k}}^{2}+\mathrm{c} \sum \mathrm{y}_{\mathrm{k}}+\mathrm{I}_{\mathrm{x}} \sum \mathrm{y}_{\mathrm{k}} \Gamma_{1}+\mathrm{I}_{\mathrm{y}} \sum \mathrm{y}_{\mathrm{k}} \Gamma_{2}+\mathrm{I}_{\mathrm{z}} \sum \mathrm{y}_{\mathrm{k}} \Gamma_{3}=\sum \mathrm{y}_{\mathrm{k}} \Delta \mathrm{Z}_{\mathrm{k}}, \\
\mathrm{a} \sum \mathrm{x}_{\mathrm{k}}+\mathrm{b} \sum \mathrm{y}_{\mathrm{k}}+\mathrm{cm}+\mathrm{I}_{\mathrm{x}} \sum \Gamma_{1}+\mathrm{I}_{\mathrm{y}} \sum \Gamma_{2}+\mathrm{I}_{\mathrm{z}} \sum \Gamma_{3}=\sum \Delta \mathrm{Z}_{\mathrm{k}}, \\
\mathrm{a} \sum \Gamma_{1} \mathrm{x}_{\mathrm{k}}+\mathrm{b} \sum \Gamma_{1} \mathrm{y}_{\mathrm{k}}+\mathrm{c} \sum \Gamma_{1}+\mathrm{I}_{\mathrm{x}} \sum \Gamma_{1}^{2}+\mathrm{I}_{\mathrm{y}} \sum \Gamma_{1} \Gamma_{2}+\mathrm{I}_{\mathrm{z}} \sum \Gamma_{1} \Gamma_{3}=\sum \Gamma_{1} \Delta \mathrm{Z}_{\mathrm{k}}, \\
\mathrm{a} \sum \Gamma_{2} \mathrm{x}_{\mathrm{k}}+\mathrm{b} \sum \Gamma_{2} \mathrm{y}_{\mathrm{k}}+\mathrm{c} \sum \Gamma_{2}+\mathrm{I}_{\mathrm{x}} \sum \Gamma_{2} \Gamma_{1}+\mathrm{I}_{\mathrm{y}} \sum \Gamma_{2}^{2}+\mathrm{I}_{\mathrm{z}} \sum \Gamma_{2} \Gamma_{3}=\sum \Gamma_{2} \Delta \mathrm{Z}_{\mathrm{k}}, \\
\mathrm{a} \sum \Gamma_{3} \mathrm{x}_{\mathrm{k}}+\mathrm{b} \sum \Gamma_{3} \mathrm{y}_{\mathrm{k}}+\mathrm{c} \sum \Gamma_{3}+\mathrm{I}_{\mathrm{x}} \sum \Gamma_{3} \Gamma_{1}+\mathrm{I}_{\mathrm{y}} \sum \Gamma_{3} \Gamma_{2}+\mathrm{I}_{\mathrm{z}} \sum \Gamma_{3}^{2}=\sum \Gamma_{3} \Delta \mathrm{Z}_{\mathrm{k}} .
\end{gathered}
$$

Матрица коэффициентов системы является симметричной, на ее главной диагонали расположены заведомо положительные числа. Решая систему стандартными методами, например, методом исключения Гаусса, получим значения избыточных плотностей объектов и коэффициенты, описывающие региональный фон.

К данной задаче можно подойти и по-другому: как к решению переопределенной системы линейных уравнений относительно тех же параметров:

$$
\begin{gathered}
\mathrm{ax}_{1}+\mathrm{by}_{1}+\mathrm{c}+\mathrm{I}_{\mathrm{x}} \Gamma_{1}\left(\mathrm{x}_{1}, \mathrm{y}_{1}, \mathrm{z}_{1}\right)+\mathrm{I}_{\mathrm{y}} \Gamma_{2}\left(\mathrm{x}_{1}, \mathrm{y}_{1}, \mathrm{z}_{1}\right)+\mathrm{I}_{\mathrm{z}} \Gamma_{3}\left(\mathrm{x}_{1}, \mathrm{y}_{1}, \mathrm{z}_{1}\right)=\Delta \mathrm{Z}_{\mathrm{m}}\left(\mathrm{x}_{1}, \mathrm{y}_{1}, \mathrm{z}_{1}\right) \\
\mathrm{ax}_{2}+\mathrm{by}_{2}+\mathrm{c}+\mathrm{I}_{\mathrm{x}} \Gamma_{1}\left(\mathrm{x}_{2}, \mathrm{y}_{2}, \mathrm{z}_{2}\right)+\mathrm{I}_{\mathrm{y}} \Gamma_{2}\left(\mathrm{x}_{2}, \mathrm{y}_{2}, \mathrm{z}_{2}\right)+\mathrm{I}_{\mathrm{z}} \Gamma_{3}\left(\mathrm{x}_{2}, \mathrm{y}_{2}, \mathrm{z}_{2}\right)=\Delta \mathrm{Z}_{\mathrm{m}}\left(\mathrm{x}_{2}, \mathrm{y}_{2}, \mathrm{z}_{2}\right) \\
\ldots \ldots \ldots \ldots \ldots \ldots \ldots \ldots \ldots \ldots \ldots \ldots \ldots \ldots \ldots \ldots \ldots \ldots \ldots \ldots \ldots \ldots \ldots \ldots \ldots \ldots \ldots \ldots \ldots \ldots \ldots \ldots \ldots \ldots \ldots \ldots \\
\mathrm{ax}_{\mathrm{m}}+\mathrm{by}_{\mathrm{m}}+\mathrm{c}+\mathrm{I}_{\mathrm{x}} \Gamma_{1}\left(\mathrm{x}_{\mathrm{m}}, \mathrm{y}_{\mathrm{m}}, \mathrm{z}_{\mathrm{m}}\right)+\mathrm{I}_{\mathrm{y}} \Gamma_{2}\left(\mathrm{x}_{\mathrm{m}}, \mathrm{y}_{\mathrm{m}}, \mathrm{z}_{\mathrm{m}}\right)+\mathrm{I}_{\mathrm{z}} \Gamma_{3}\left(\mathrm{x}_{\mathrm{m}}, \mathrm{y}_{\mathrm{m}}, \mathrm{z}_{\mathrm{m}}\right)=\Delta \mathrm{Z}_{\mathrm{m}}\left(\mathrm{x}_{\mathrm{m}}, \mathrm{y}_{\mathrm{m}}, \mathrm{z}_{\mathrm{m}}\right) .
\end{gathered}
$$

$\mathrm{B}$ общем случае подобное определение $\mathrm{n}$ параметров по $\mathrm{m}$ точкам наблюдения сводится к решению системы линейных уравнений

$$
\mathrm{Ap}=\mathrm{u},
$$

где $\mathrm{A}$ - прямоугольная матрица размерами $\mathrm{m} \times \mathrm{n}$. Для ее решения надо умножить обе части матричного уравнения слева на матрицу $\mathrm{A}^{\mathrm{T}}$, транспонированную к матрице $\mathrm{A}$ :

$$
\mathrm{A}^{\mathrm{T}} \mathrm{Ap}=\mathrm{A}^{\mathrm{T}} \mathrm{u} \text {. }
$$

Матрица $\mathrm{A}^{\mathrm{T}} \mathrm{A}$ является квадратной симметричной матрицей размерами $\mathrm{n} \times \mathrm{n}$ и имеющей тот же ранг, что и матрица А. Легко убедиться, что оба рассмотренных подхода приводят к решению одной и той же системы линейных алгебраических уравнений.

Рассмотренные выше решения характерны для так называемого детерминистского подхода, но и в рамках статистического подхода, базирующегося на методе максимального правдоподобия, в случае некоррелированной помехи, распределенной по нормальному закону и имеющей нулевое математическое ожидание, решение линейной задачи подбора сводится к решению той же системы.

В рамках статистического направления матрицу коэффициентов системы нормальных уравнений $\mathrm{A}^{\mathrm{T}} \mathrm{A}$, каждый элемент которой поделен на дисперсию помехи $\varepsilon^{2}$, принято называть информационной матрицей параметров или матрицей Фишера. Важнейшим достижением статистического подхода является априорная оценка качества интерпретации, проводимая путем построения ковариационной матрицы параметров $\mathrm{B}$, являющейся обратной $к$ информационной матрице, то есть

$$
\mathrm{B}=\varepsilon^{2}\left(\mathrm{~A}^{\mathrm{T}} \mathrm{A}\right)^{-1} \text {. }
$$

Ковариационная матрица $\mathrm{B}$ является квадратной симметричной матрицей с элементами $\mathrm{B}_{\mathrm{ij}}$, причем і и ј изменяются от 1 до $\mathrm{n}$. Диагональные элементы ковариационной матрицы $\mathrm{B}_{\mathrm{ii}}$ имеют смысл дисперсии оценки і-го параметра, а квадратный корень из $\mathrm{B}_{\mathrm{ii}}$ соответственно характеризует среднеквадратическую погрешность его оценки, что дает возможность получать доверительные интервалы для каждого из определяемых параметров. Внедиагональные элементы матрицы $\mathrm{B}_{\mathrm{ij}}=\mathrm{B}_{\mathrm{ji}}$, где $\mathrm{i} \neq \mathrm{j}$, характеризуют корреляционные связи между оценками $\mathrm{i}-г о$ и j-го параметров. Они показывают, насколько устойчиво определяются данные параметры. Если их изменения влияют на аномальное поле почти одинаково, то они по этому полю будут определяться неустойчиво, так как изменение поля из-за вариации одного из параметров может компенсироваться вариацией второго. Для количественной оценки данного эффекта вводят нормированные коэффициенты корреляции: 


$$
b_{i j}=\frac{B_{i j}}{\sqrt{B_{i i}} \sqrt{B_{j j}}} .
$$

Если $b_{\mathrm{ij}}$ близок к нулю, то i-ый и j-ый параметры определяются совместно достаточно устойчиво. Если же модуль нормированных корреляционных коэффициентов близок к единице, то совместное определение данных параметров, основанное на выявлении различий их влияния на аномальное поле, становится затруднительным. Таким образом, ковариационная матрица параметров позволяет выяснить, какие из параметров определяются устойчиво, а какие неустойчиво, а также оценить предельно возможную точность оценки параметров. Вычисление элементов ковариационной матрицы путем обращения информационной матрицы Фишера может вестись любыми из известных способов, разработанных в линейной алгебре.

\section{ЛИТЕРАТУРА}

1. Авдевич М.М., Фокин А.Ф. Методика электромоделирования потенциальных геофизических полей при оперативной интерпретации данных скважинной геофизики. Л: ОНТИ ВИТР. 1976. 62 с.

2. Агафонова М.Б., Филатов В.В. Алгоритм и программа решения прямой задачи магниторазведки при моделировании железорудных месторождений Урала // Геофизические методы поисков и разведки рудных и нерудных месторождений. Свердловск: СГИ. 1982. вып. 8. c. $89-93$.

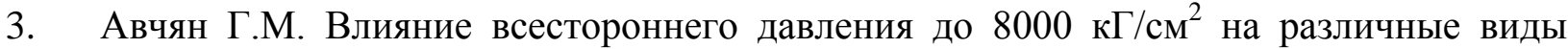
остаточной намагниченности горных пород // Известия АН СССР. Физика Земли. 1967. № 7. c. 70-76.

4. Альпин Л.М. Намагничение и размагничивающий фактор // Известия вузов. Геология и разведка. 1967. № 9. с. 137-141.

5. Альпин Л.М., Даев Д.С., Каринский А.Д. Теория полей, применяемых в разведочной геофизике. М.: Недра. 1985. 407 с.

6. Ангенхейстер Г., Хаальк Г., Щодро Н.Л. Магнитометрия // Прикладная геофизика. 1936. № 3. c. 6-180.

7. Андреев В.И. К вопросу о влиянии механических напряжений на магнитные свойства магнетитовой руды // Геология и геофизика. 1960. № 7. с. 129-133.

8. Антонец А.Г. Метод геомагнитных вариаций // Труды Ташкентского политехнического института. 1975. вып. 94. с. 109.

9. Антонов Ю.В., Вассерман И.С., Слюсарев С.В. Определение интенсивности намагничения железистых кварцитов КМА по наземным измерениям // Известия вузов. Геология и разведка. 1985. № 6. с. 98-101.

10. Антонов Ю.В., Винокуров С.К., Слюсарев С.В. Возможности оценки качества железистых кварцитов КМА по вариациям магнитного поля // Изв. ВУЗов. Геология и разведка. 1990. № 12. c. 74-80.

11. Антонов Ю.В., Слюсарев С.В. Интерпретация магнитных аномалий над анизотропно намагниченными пластами // Геофизические методы поисков и разведки рудных и нерудных месторождений. Свердловск: СГИ. 1984. вып. 10. с. 25-30.

12. Бабаянц П.С., Блох Ю.И., Трусов А.А. Изучение рельефа поверхности кристаллического фундамента по данным магниторазведки // Геофизика. 2003. № 4. с. 37-40.

13. Бабаянц П.С., Блох Ю.И., Трусов А.А. Изучение строения кристаллического основания платформенных областей по данным магниторазведки и гравиразведки // Геофизика. 2003. № 6. c. 55-58. 
Ю.И. Блох

Теоретические основы комплексной магниторазведки

14. Бабаянц П.С., Блох Ю.И., Трусов А.А. Возможности структурно-вещественного картирования по данным магниторазведки и гравиразведки в пакете программ СИГМА-3D // Геофизический вестник. 2004. № 3 с. 11-15.

15. Бабаянц П.С., Блох Ю.И., Трусов А.А. Аномальные поля фрактальных моделей геологических объектов // Геофизика. 2005. № 5. с. 42-46.

16. Бабаянц П.С., Блох Ю.И., Трусов А.А. Интерактивные технологии локальной количественной экспресс-интерпретации потенциальных полей // Геофизика. 2006. № 1. с. 5659.

17. Белоголов В.Т., Логинов В.Е. Использование электронно-вычислительных машин при работах, связанных с поисками и разведкой железорудных месторождений Западной Сибири // ОНТИ ВИЭМС. Информационное сообщение. 1968. № 61.

18. Белоголов В.Т., Соболев В.В. Вычисление среднего значения намагниченности тел сложной формы // Труды СНИИГГИМС. 1971. вып. 136. с. 47-49.

19. Билибин С.И., Мараев И.А., Мухина Н.И., Плюснин М.И., Семенов В.Ю. Влияние анизотропии однородных сильномагнитных сред на результаты магнитного каротажа // Известия вузов. Геология и разведка. 1982. № 11. с. 129-133.

20. Блох Ю.И. Взаимодействие горизонтальных круговых цилиндров в однородном, гармонически меняющемся магнитном поле // Известия вузов. Геология и разведка. 1972. № 6. c. $120-124$.

21. Блох Ю.И. Взаимное влияние намагничивающихся круговых цилиндров // Известия вузов. Геология и разведка. 1974. № 3. с. 127-134.

22. Блох Ю.И. Взаимное влияние намагничивающихся эллиптических цилиндров и эллипсоидов вращения // Известия вузов. Геология и разведка. 1974. № 4. с. 120-125.

23. Блох Ю.И. О решении прямой задачи теории магниторазведки для тел произвольной формы с учетом размагничивания // Известия вузов. Геология и разведка. 1975. № 1. с. 100-108.

24. Блох Ю.И. Расчет магнитного поля двумерных анизотропных объектов произвольного сечения с учетом размагничивания // Известия АН СССР. Физика Земли. 1980. № 2. c. 65-72.

25. Блох Ю.И. Совместная интерпретация данных магниторазведки и метода незаземленной петли при определении магнитных свойств магнетитовых руд // Известия АН СССР. Физика Земли. 1981. № 11. с. 74-81.

26. Блох Ю.И. Учет размагничивания при решении прямой задачи магниторазведки для трехмерных изотропных объектов // Известия АН СССР. Физика Земли. 1983. № 5. с. 113-117.

27. Блох Ю.И. Влияние размагничивания на магнитные аномалии интрузивных объектов // Известия вузов. Геология и разведка. 1984. № 3. с. 122-124.

28. Блох Ю.И. Возможности интерпретации магнитных аномалий с учетом размагничивания // Известия АН СССР. Физика Земли. 1987. № 4. с. 56-62.

29. Блох Ю.И. Решение прямой задачи магниторазведки для трехмерных анизотропных геологических объектов с учетом размагничивания // Известия АН СССР. Физика Земли. 1987. № 12. c. 49-55.

30. Блох Ю.И. Комплексирование методов интерпретации при определении природы магнитных аномалий // Известия АН СССР. Физика Земли. 1991. № 3. с. 36-42.

31. Блох Ю.И. Анизотропия магнитной восприимчивости пачки пластов // Известия вузов. Геология и разведка. 1993. № 2. с. 115-120.

32. Блох Ю.И. Решение прямых задач гравиразведки и магниторазведки. М: Издательство МГГА. 1993. 79 с.

33. Блох Ю.И. Обнаружение и разделение гравитационных и магнитных аномалий. М: Издательство МГГА. 1995.80 с.

34. Блох Ю.И. Основные закономерности намагничения многоугольных цилиндров и многогранников // Физика Земли. 1997. № 1. с 59-63.

35. Блох Ю.И. Количественная интерпретация гравитационных и магнитных аномалий. М: Издательство МГГА. 1998. 88 с. 
36. Блох Ю.И. Намагничение моделей, аппроксимирующих геологические объекты // Геофизика. 1998. № 2. с. 42-44.

37. Блох Ю.И. Проблема адекватности интерпретационных моделей в гравиразведке и магниторазведке // Геофизический вестник. 2004. № 6 с. 10-15.

38. Блох Ю.И. Комплексная магниторазведка - возможности и проблемы // Вопросы теории и практики геологической интерпретации гравитационных, магнитных и электрических полей: Материалы 36-й сессии Международного семинара им. Д.Г. Успенского. Казань: Издательство Казанского государственного университета. 2009. с. 58-60.

39. Блох Ю. И. Интерпретация гравитационных и магнитных аномалий. Учебное пособие. М., 2009. http://sigma3d.com/content/view/24/2/.

40. Блох Ю.И. Аномалии взаимовлияния сближенных сильномагнитных объектов // Вопросы теории и практики геологической интерпретации гравитационных, магнитных и электрических полей: Материалы 37-й сессии Международного семинара им. Д.Г. Успенского. М: ИФЗ РАН. 2010. с. 58-62.

41. Блох Ю.И. Томографические возможности комплексной магниторазведки // Вопросы теории и практики геологической интерпретации геофизических полей: Материалы 38-й сессии Международного научного семинара им. Д.Г. Успенского. Пермь: ГИ УрО РАН. 2011. с. 29-32.

42. Блох Ю.И. Возможности комплексной магниторазведки при определении магнитной восприимчивости анизотропных геологических объектов в естественном залегании // Геофизика. 2011. № 3. с. 43-47.

43. Блох Ю.И., Гаранский Е.М., Доброхотова И.А., Ренард И.В. Интерпретация результатов измерений методом незаземленной петли над крутопадающими намагничивающимися пластами // Известия вузов. Геология и разведка. 1979. № 8. с. 62-68.

44. Блох Ю.И., Гаранский Е.М., Доброхотова И.А., Ренард И.В., Якубовский Ю.В. Комплексная интерпретация данных метода незаземленной петли и магниторазведки при разведке месторождений железистых кварцитов // Геология и геофизика. 1984. № 1. с. 104-109.

45. Блох Ю.И., Гаранский Е.М., Доброхотова И.А., Ренард И.В., Якубовский Ю.В. Низкочастотная индуктивная электроразведка при поисках и разведке магнитных руд. М: Недра. 1986. 192 с.

46. Блох Ю.И., Гафарова М.Г., Тягны-Рядно А.Р. Влияние размагничивания на магнитное поле крутопадающих полубесконечных пластов и штоков // Известия вузов. Геология и разведка. 1984. № 5. с. 115-117.

47. Блох Ю.И., Доброхотова И.А., Кириллов Р.Н., Никитина Т.А., Ренард И.В., Утепов С.К., Якубовский Ю.В. Применение методов незаземленной петли и магниторазведки для расшифровки природы отрицательных магнитных аномалий на железорудном месторождении Кумдыколь (Центральный Казахстан) // Известия вузов. Геология и разведка. 1986. № 8. c. 66-73.

48. Блох Ю.И., Доброхотова И.А., Овешников С.3., Ренард И.В. Поиски магнитных объектов под перекрывающими неоднородными магнитными породами с помощью метода незаземленной петли // Известия вузов. Геология и разведка. 1986. № 12. с. 65-70.

49. Блох Ю.И., Доброхотова И.А., Ренард И.В. Магнитные аномалии, возникающие в результате грозовых разрядов на месторождениях магнетитовых руд // Физика Земли. 1996. № 10 . с $68-74$.

50. Блох Ю.И., Каплун Д.В., Коняев О.Н. Возможности интерпретации потенциальных полей методами особых точек в интегрированной системе "Сингуляр" // Известия вузов. Геология и разведка. 1993. № 6. с. 123-127.

51. Блох Ю.И., Мещаненко Ю.В. Комплексные микрогеофизические исследования магнитных свойств траппов // Известия вузов. Геология и разведка. 1990. № 11. с. 139-142.

52. Блох Ю.И., Светлицкая О.Э. О намагничении сильномагнитных пластов // Известия вузов. Геология и разведка. 1987. № 11. с. 106-110.

53. Блох Ю.И., Трусов А.А. Программа «IGLA» для интерактивной экспрессинтерпретации локальных гравитационных и магнитных аномалий // Вопросы теории и 
практики геологической интерпретации гравитационных, магнитных и электрических полей: материалы 34-й сессии международного семинара им. Д.Г.Успенского. М: ИФЗ РАН. 2007. c. 36-38.

54. Бондаренко С.И., Биезайс Я.Я., Веркин Б.И., Стогний В.В., Фенченко В.Н., Шеремет В.И. Опыт работы со сверхпроводниковыми магнитовариационными станциями // Геология и геофизика. 1980. № 11. с. 94-101.

55. Бородаева Н.М., Соловьев О.А. Интерпретация магнитных аномалий при методе искусственного подмагничивания // Геология и геофизика. 1964. № 6. с. 153-156.

56. Васильев Р.Т., Князев Л.И. Метод искусственного подмагничивания двухдипольной установкой // Экспресс-информация. ВИЭМС. Сер. Региональная, разведочная и промысловая геофизика. М: ВИЭМС. 1977. № 7. с. 1-8.

57. Вейнберг А.К., Квачевский О.А. Коэффициенты размагничивания тел кубической формы // Геофизическая аппаратура. 1981. вып. 72. с. 12-18.

58. Великин А.Б., Франтов Г.С. Электромагнитные поля, применяемые в индукционных методах электроразведки. Л: Гостоптехиздат. 1962. 352 с.

59. Велихов Е.П., Иванов Н.А., Стадухин В.Д., Волков Ю.М., Вершинин В.С. Опыт определения магнитной восприимчивости горных пород в естественном залегании при эксперименте с МГД-генератором // ДАН СССР. 1977. т. 237. № 3. с. 541-543.

60. Вешев А.В., Любцева Е.Ф., Самосюк Г.П. Определение эффективных параметров среды в поле конечного заземленного кабеля. I // Ученые записки ЛГУ. Серия физических и геологических наук. 1963. вып. 14. с. 3-63.

61. Вешев А.В., Любцева Е.Ф., Самосюк Г.П. Определение эффективных параметров среды в поле конечного заземленного кабеля. II // Ученые записки ЛГУ. Серия физических и геологических наук. 1964. вып. 15. с.174-249.

62. Вешев А.В., Любцева Е.Ф., Самосюк Г.П. Нормальное поле незаземленной петли // Ученые записки ЛГУ Серия физических и геологических наук. 1967. вып. 17. с. 23-56.

63. Винничук Н.Н., Костров Н.П., Ратушняк А.Н. Применение объемных интегральных уравнений в задачах магнитометрии. Екатеринбург: УрО РАН. 1999. 52 с.

64. Вонсовский С.В. Магнетизм. М: Наука. 1971. 1032 с.

65. Воробьев М.В. Использование параметра магнитных свойств коэрцитивной силы в поисках магнетитовых месторождений // Информационный сборник Запсибгеофизтреста. Новокузнецк. 1957. № 2.

66. Воскобойников Г.М. О вычислении стационарных электромагнитных полей в некоторых кусочно-однородных средах // Известия АН СССР. Физика Земли. 1973. № 9. с. 63 75.

67. Воскобойников Г.М., Гуревич Ю.М., Збыковская Т.А., Мартышко П.С., Пруткин И.Л., Цирульский А.В. Прямые и обратные задачи метода искусственного подмагничивания // Теория и методика интерпретации гравимагнитных полей. Киев: Наукова Думка. 1981. с. 38-51.

68. Гаранский Е.М., Доброхотова И.А., Ренард И.В., Якубовский Ю.В. Применение низкочастотных индуктивных методов электроразведки для поисков месторождений магнитных руд. Обзор. Региональная, разведочная и промысловая геофизика. М: ВИЭМС. 1976. 57 с.

69. Гольцман Ф.М. Статистические модели интерпретации. М.: Наука. 1971. 328 с.

70. Гольцман Ф.М., Калинина Т.Б. Статистическая интерпретация магнитных и гравитационных аномалий. Л.: Недра. 1983. 248 с.

71. Грабовский М.А. Изменение магнитных свойств магнетита под действием механических напряжений // Известия АН СССР. Серия географическая и геофизическая. 1949. т. 13. № 2. с. 143-150.

72. Грабовский М.А., Пархоменко Э.И. Об изменении магнитных свойств магнетитов под действием больших сжимающих напряжений // Изв. АН СССР. Сер. геофиз. 1953. № 5. c. $405-417$.

73. Гринберг Г.А. Избранные вопросы математической теории электрических и 
магнитных явлений. М-Л: Издательство АН СССР. 1948. 728 с.

74. Гуревич Ю.М., Яковлев А.А., Ольшанецкая И.Л. Зондирование МИП с длинным кабелем и квадратной рамкой // Вопросы метода искусственного подмагничивания. Свердловск: Ин-т геофиз. УрО АН СССР. 1991. с. 67-76. (Деп. ВИНИТИ. № 3669-В91).

75. Давыдов А.Я., Молчанов А.Н. Анализ магнитных аномалий методом искусственного подмагничивания // Разведка и охрана недр. 1978. № 2. с. 55-56.

76. Девицын В.М., Лапина М.И., Шнеерсон Б.Л. О влиянии неоднородной намагниченности тела постоянной восприимчивости на результаты интерпретации магнитных аномалий простейшими методами // Известия АН СССР. Серия геофизическая. 1961. № 3. c. $428-432$.

77. Доброхотова И.А. Горизонтальный эллиптический цилиндр в переменном электромагнитном поле // Известия вузов. Геология и разведка. 1968. № 9.

78. Доброхотова И.А., Юдин М.Н. Влияние магнитной проницаемости на результаты магнитотеллурических зондирований // Известия вузов. Геология и разведка. 1981. № 6. с. 99106.

79. Доброхотова И.А., Якубовский Ю.В. О возможностях и результате применения индуктивных методов электроразведки на месторождениях магнитных руд // Разведочная геофизика. 1970. вып. 39. с. 86-89.

80. Дучков А.Д., Казанцев С.А., Велинский В.В. и др. Оценка разрешающей способности метода искусственного подмагничивания по результатам работ на Угловой аномалии // Геология и геофизика. 1979. № 9. с. 142-148.

81. Евдокимов И.М., Селезнева Г.В., Терехова Р.В. Геофизические методы при поисках и разведке медноколчеданных и магнетитовых месторождений // Обзор ВИЭМС. Разведочная геофизика. 1984. 52 с.

82. Евдокимов И.М., Селезнева Г.В., Хахалина Т.А. О возможности использования индуктивности петли для электроразведочных целей // Электрометрические исследования при поисках и разведке рудных месторождений. Свердловск. 1977. с. 121-123.

83. Ермохин К.М. Расчет полей постоянного тока в трехмерных неоднородных средах. Деп. в ВИНИТИ. 19.09.1985. № 6732-1385. 10 с.

84. Завойский В.Н. Вычисление магнитных полей от анизотропных трехмерных тел в задачах магниторазведки // Известия АН СССР. Физика Земли. 1978. № 1. с. 76-85.

85. Захаров В.Х., Колесник А.В. Определение магнитной проницаемости горных пород дипольными индуктивными установками с амплитудно-фазовыми измерениями // Записки ЛГИ. 1976. т. 69. № 2. с. 69-76.

86. Захарченко В.Ф., Стадухин В.Д., Вершинин В.С., Ерошенко Н.А. Дипольный вариант метода искусственного подмагничивания // Теория и практика метода искусственного подмагничивания при поисках железорудных месторождений. Свердловск, 1982. с. 3-8.

87. Зоммерфельд А. Электродинамика. М.: Издательство иностранной литературы. 1958. $501 \mathrm{c}$.

88. Зидаров Д.П. О решении некоторых обратных задач потенциальных полей и его применения к вопросам геофизики. София: Издательство Болгарской АН. 1968. 154 с.

89. Иванов Н.А., Стадухин В.Д. Применение метода искусственного подмагничивания для установления природы магнитных аномалий // Разведка и охрана недр. 1974. № 7. с. 36-39.

90. Иванов Н.А., Стадухин В.Д., Вершинин В.С. Расчет первичного поля на ЭВМ в методе искусственного подмагничивания // Применение математических методов и ЭВМ при обработке информации на геологоразведочных работах. Свердловск. 1977. с. 27-28.

91. Иванов Н.А., Стадухин В.Д., Улитина Г.Г. Применение метода искусственного подмагничивания для оценки содержания железа в разрабатываемых открытым способом магнетитовых и титаномагнетитовых рудах железорудных месторождений // Разведочная геофизика. Свердловск. 1966. с. 172-178. (Труды Свердловского горного института. Вып. 47).

92. Иванов Н.А., Стадухин В.Д., Улитина Г.Г. Определение содержания железа в рудах магнетитовых месторождений методом искусственного подмагничивания // Вопросы методики 
опробования месторождений полезных ископаемых при разведке и эксплуатации. Свердловск. 1969. с. 63-68.

93. Иванов Н.А., Шапиро В.А. Особенности остаточной намагниченности магнетитов Покровско-Уральского месторождения // Настоящее и прошлое магнитного поля Земли. М: Наука. 1965. с. 183-186.

94. Иванов Н.А., Шапиро В.А. Изменение остаточной намагниченности магнетита после взрыва // ДАН СССР. 1967. т. 177. № 2. с. 322-324.

95. Иванов Н.А., Шапиро В.А. Характеристика динамической намагниченности, создаваемой ударами в образцах естественных ферромагнетиков // Известия АН СССР. Физика Земли. 1969. № 5. с. 50-60.

96. Калашников А.Г. Метод определения магнитной восприимчивости горных пород в естественных условиях // ДАН СССР. 1948. т. 63. № 6. с. 657-660.

97. Калашников А.Г. Определение магнитной восприимчивости горных пород в полевых условиях // Известия АН СССР. Серия геофизическая. 1954. № 5. с. 415-423.

98. Калашников А.Г. Моделирование аномалий магнитного и гравитационного полей и изучение их при помощи автоматического магнитного полемера // Известия АН СССР. Серия геофизическая. 1954. № 6. с. 546-553.

99. Калашников А.Г. Магнитные свойства неоднородно намагниченных призм. I // Известия АН СССР. Серия геофизическая. 1956. № 12. с. 1369-1383.

100. Калашников А.Г. Распределение остаточной намагниченности в кубах и цилиндрах из горных пород // Известия АН СССР. Серия геофизическая. 1958. № 4. с. 550-553.

101. Калашников А.Г. Магнитные свойства неоднородно намагниченных призм. II // Известия АН СССР. Серия геофизическая. 1958. № 11. с. 1335-1341.

102. Калашников А.Г., Капица С.П. Магнитная восприимчивость горных пород при упругих напряжениях // ДАН СССР. 1952. т. 86. № 3. с. 521-523.

103. Каменская Т.Я. Изучение вариаций магнитного поля Земли для определения геологической природы магнитных аномалий // Вестник Западно-Сибирского и Новосибирского геологических управлений. Томск: Издателство Томского университета. 1960. № 2. c. 30-33.

104. Каменская Т.Я. О возможности использования вариаций геомагнитного поля для определения природы магнитных аномалий // Практическое применение геофизических методов разведки / Под ред. Д.С. Микова. Томск. 1966. с. 223-227.

105. Каменская Т.Я. Определение природы слабых магнитных аномалий с помощью наблюдений суточных вариаций геомагнитного поля // Информационное сообщение ОНТИ ВИЭМС. Сер. регион., разв. и промысл. геофизика. 1966. № 3. с. 3-17.

106. Капица С.П. Магнитные свойства изверженных горных пород при механических напряжениях // Известия АН СССР. Серия геофизическая. 1955. № 6. с. 489-504.

107. Кириллов В.М. Некоторые результаты приложения метода вариаций магнитного поля Земли // Разведка и охрана недр. 1969. № 2. с. 60-62.

108. Кириллов В.М., Апарин В.П. О влиянии процесса бурения на естественную остаточную намагниченность осадочных пород Кузбасса // Геология и геофизика. 1972. № 2. c. $125-132$.

109. Колюбакин В.В., Лапина М.И. Обзор способов решения прямой и обратной задач магнитной разведки // Труды ИФЗ АН СССР. 1960. 13 (180). 362 с.

110. Константинов Г.Н. Математическое моделирование при решении поисковоразведочных задач на железо // Математическое и физическое моделирование железорудных месторождений и рудных полей. Новосибирск: СНИИГГИМС. 1983. с. 18-33.

111. Константинов Г.Н., Константинова Л.С. Опыт применения метода моделирования для интерпретации магнитного поля Таштагольского железорудного месторождения // Геология и геофизика. 1967. № 12. с. 86-96.

112. Константинов Г.Н., Константинова Л.С. Моделирование в рудной магниторазведке: Методическое пособие. Новосибирск: СНИИГГиМС. 1971. 76 с. 
113. Константинов Г.Н., Константинова Л.С. Методика и результаты прогнозной оценки Ималыкского железорудного района Чаро-Токкинской зоны по геолого-геофизическим данным // Труды СНИИГГИМС. 1976. вып. 238. с. 9-18.

114. Константинов Г.Н., Константинова Л.С., Козин С.Г., Марков В.В. Методические рекомендации по математическому моделированию железорудных месторождений по магнитным полям на ранних стадиях геологоразведочных работ. Новосибирск: СНИИГГиМС. 1988. $130 \mathrm{c}$.

115. Копаев В.В., Лапина М.И., Распопов О.М. Вариационный метод определения магнитных свойств сильномагнитных горных пород // Известия АН СССР. Серия геофизическая. 1961. № 9. с. 1354-1362.

116. Корольков А.Л. Прибор для измерения магнитного потока индукции и применения его в лаборатории // Журнал Русского физико-химического общества. Физический отдел. 1908. т. 40. вып. 9. с. 388-408.

117. Крутиховская 3.А., Завойский В.Н., Подолянко С.М., Савенко Б.Я. Намагниченность пород железорудных формаций Большого Кривого Рога и КМА. Киев: Наукова думка. 1964. 179 c.

118. Крутиховская 3.А., Силина И.М., Завойский В.Н. и др. Структура фундамента и железорудные месторождения северного склона Украинского щита. Киев: Наукова думка. 1975. $231 \mathrm{c}$.

119. Лаврентьев М.М. О некоторых некорректных задачах математической физики. Новосибирск: СО АН СССР. 1962. 92 с.

120. Лапин С.С. Магнетитовые рудные тела, их строение и магнитные свойства // Труды ИГиГ СО АН СССР: Вып. 212. Новосибирск: Наука. Сибирское отделение. 1976. 236 с.

121. Лапина М.И. Об использовании вариаций геомагнитного поля для определения магнитной восприимчивости сильномагнитных горных пород в условиях естественного залегания // Известия АН СССР. Серия геофизическая. 1961. № 3. с. 418-422.

122. Ларионов В.А. К вопросу об определении в полевых условиях величины отношения остаточного намагничения к индуктивному // Геология и геофизика. 1961. № 4. с. 107-109.

123. Лейст Э.Е. Курская магнитная аномалия / Материалы по исследованию Курской магнитной аномалии. 1921. вып. 2.72 с.

124. Лучицкий А.И. Прямые задачи в теории метода искусственного подмагничивания // Теория и методика интерпретации гравитационных и магнитных аномалий. М: ИФЗ АН СССР. 1979. c. $126-145$.

125. Маергойз И.Д. Итерационные методы расчета статических полей в неоднородных, анизотропных и нелинейных средах. Киев: Наукова думка. 1979. 210 с.

126. Маергойз И.Д., Тозони О.В. Использование потенциала двойного слоя для расчета статических магнитных полей в кусочно-однородной среде // Кибернетика и вычислительная техника. 1971. вып. 10. с. 88-94.

127. Малкин Н.Р. О намагничивании прямоугольной призмы в однородном поле // Труды Центральной обсерватории. 1934. т. 1. № 3.

128. Малкин Н.Р. О намагничении двух бесконечных цилиндров // Труды ГГО. 1936. вып. 11.

129. Мартышко П.С. Некоторые вопросы теории и алгоритмы решения задач метода искусственного подмагничивания. Препринт Института геофизики УНЦ АН СССР. Свердловск. 1982. $31 \mathrm{c}$.

130. Мартышко П.С. Решение задач метода искусственного подмагничивания (МИП) в двух- и трехмерном случаях // Теория и практика интерпретации гравитационных и магнитных полей в СССР. Киев: Наукова Думка. 1983. с. 198-204.

131. Мартышко П.С., Пруткин И.Л. О решении прямой и обратной задач магниторазведки // Геофизический журнал. 1982. т. 4. № 6. с. 39-49.

132. Мартышко П.С., Пьянков В.А. О единственности решения обратной задачи теории потенциала в методе подмагничивания вращающимся полем $\left(\mathrm{S}_{\mathrm{q}}\right.$-вариации) // Вопросы теории и 
результаты применения методов интерпретации и моделирования геофизических полей. Свердловск. 1989. с. 13-18.

133. Мохова Е.Н. Расчет намагниченности призмы при постоянной восприимчивости // Известия АН СССР. Серия геофизическая. 1957. № 5. с. 680-682.

134. Мохова Е.Н. Прямоугольная призма постоянной восприимчивости в однородном магнитном поле // Известия АН СССР. Серия геофизическая. 1958. № 3. с. 387-390.

135. Муратов Р.3. Потенциалы эллипсоида. М.: Атомиздат. 1976. 144 с.

136. Нагата Т. Магнетизм горных пород. М: Мир. 1965. 348 с.

137. Нгуен Дык Чак. Эллипсоид вращения в однородном гармонически меняющемся магнитном поле // Известия вузов. Геология и разведка. 1972. № 6. с. 126-132.

138. Нусипов Е.Н. Общее решение прямой задачи магниторазведки для тел произвольной формы с учетом размагничивания // Вопросы прикладной геофизики. Алма-Ата. 1979. с. 25-38.

139. Овчинников В.Н. Метод искусственного подмагничивания с однопетлевой намагничивающей рамкой // Вопросы метода искусственного подмагничивания. Свердловск: Ин-т геофиз. УрО АН СССР. 1991. с. 57-66. (Деп. ВИНИТИ. № 3669-В91).

140. Овчинников В.Н., Зыков В.М. Измерительно-преобразовательный тракт МИП // Теория и практика метода искусственного подмагничивания при поисках железорудных месторождений // Под ред. В.Ф. Захарченко и А.Н. Бахвалова. Свердловск: Ин-т геофиз. УНЦ АН СССР. 1982. с. 28-33.

141. Палеомагнитология / Храмов А.Н., Гончаров Р.А., Комиссарова Р.А. и др. Л: Недра. $1982.312 \mathrm{c}$.

142. Паркинсон У. Введение в геомагнетизм. М: Мир. 1986. 528 с.

143. Пасальский П.Т. Об изучении распределения магнетизма на земной поверхности. Одесса. 1901. 547 с.

144. Пеккер И.И. Расчет магнитных систем методом интегрирования по источникам поля // Известия вузов. Электромеханика. 1964. № 9. с. 1047-1051.

145. Пеккер И.И. К расчету магнитных систем методом интегрирования по источникам поля // Известия вузов. Электромеханика. 1968. № 9. с. 940-943.

146. Попов А.А. Намагниченность магнетита в зависимости от содержания его в породах и рудах // Методика и техника разведки. 1970. вып. 68. с. 51-60.

147. Портнов В.С., Борисенко Ю.Н., Рыжий Б.П. Оценка анизотропии магнитной восприимчивости системы намагниченных трехмерных тел по их взаимовлиянию // Геофизические методы поисков и разведки рудных и нерудных месторождений. Свердловск. 1985. вып. 11. с. 35-40.

148. Прис Г.В. Электромагнитное поле круглой горизонтальной рамки, расположенной над проводящим полупространством // Известия АН СССР. Физика Земли. 1965. № 4. с. 73-82.

149. Пруткин И.Л., Цирульский А.В. О решении трехмерной обратной задачи магниторазведки // Известия АН СССР. Физика Земли. 1984. № 6. с. 79-85.

150. Ремпель Г.Г., Филатов В.А. Оценка вертикальных размеров и глубины до поверхности возмущающего тела по данным метода искусственного подмагничивания // Геология и геофизика. 1970. № 10. с. 106-109.

151. Ренард И.В. Изучение влияния магнитных свойств горных пород и руд на переменные электромагнитные поля звуковой частоты (применительно к индуктивным методам электроразведки). Автореферат диссертации на соискание ученой степени кандидата технических наук. М: МГРИ. 1972.

152. Светлов А.И., Строганов О.Г. Решение плоской магнитной задачи для некоторых миогосвязных областей // Труды ГГО. 1939. вып. 29. с. 18-49.

153. Светов Б.С. Основы геоэлектрики. М: Издательство ЛКИ. 2008. 656 с.

154. Слудский Ф.А. Об уклонении отвесных линий. Диссертация на степень магистра астрономии. М: Университетская типография. 1863. 53 с.

155. Случановский А. К вопросу о намагничении изотропного и однородного тела // Труды Физико-математического института им. В.А. Стеклова. 1934. т. 5. с. 371-382. 
156. Соболев В.В., Белоголов В.Т. Вычисление интенсивности намагничения горных пород в однородном намагничивающем поле с учетом размагничивания // Труды СНИИГГИМС. 1968. вып. 73. с. 3-7.

157. Соловьев О.А. К вопросу о разделении магнитных аномалий на рудные и безрудные // Геология и геофизика. 1960. № 9. с. 93-99.

158. Стадухин В.Д. Определение магнитной восприимчивости горных пород в естественном залегании по измерению напряженности магнитного поля в центре квадратной рамки // Геофиз. сб. Свердловск. 1962. № 3. с. 79-84. (Труды Института геофизики УФ АН СССР. Вып. 2).

159. Стадухин В.Д. К определению магнитной восприимчивости горных пород в естественном залегании по измерению магнитного потока квадратной рамки // Геофизический сборник. Свердловск. 1962. № 3. с. 85-90. (Труды Института геофизики. УФ АН СССР. Вып. 2).

160. Стадухин В.Д. Магнитное профилирование и зондирование незаземленными рамками с постоянным током // Электромагнитное зондирование и магнито-теллурические методы разведки. Л: Издательство Ленинградского университета. 1963. с. 171-179.

161. Стадухин В.Д. О возможности применения Т-магнитометра в методе искусственного подмагничивания // Теория и практика магнитометрии. Геофизический сборник. Свердловск. 1968. № 7. c. 95-98.

162. Стадухин В.Д. Определение магнитной восприимчивости пород и руд по измерению напряженности магнитного поля в скважине при искусственном подмагничивании с целью выявления и оценки железорудных месторождений // Известия АН СССР. Серия геофизическая. 1963. № 9. с. 1381-1385.

163. Стадухин В.Д. Палетки для приближенного расчета вторичного поля в методе искусственного подмагничивания // Теория и практика магнитометрии. Геофизический сборник. Свердловск. 1968. № 7. с. 81-94.

164. Стадухин В.Д. Определение радиуса и магнитной восприимчивости сильномагнитного горизонтального кругового цилиндра // Методика магнитных измерений и интерпретаций в геофизике. Свердловск. 1970. с. 3-6. (Труды Института геофизики УФ АН СССР. Вып. 5).

165. Стадухин В.Д., Вершинин В.С., Туранов В.Н., Шабанова Н.Н. Оценка разрешающей способности метода искусственного подмагничивания по результатам работ на Угловой аномалии // Геология и геофизика. 1979. № 9. с. 142-148.

166. Стадухин В.Д., Гуревич Ю.М., Захарченко В.Ф., Вершинин В.С., Туранов В.Н., Жиромский Б.В., Збыковская Т.А. Метод искусственного подмагничивания с измерением магнитного поля вне рамки // Теория и практика метода искусственного подмагничивания при поисках железорудных месторождений. Свердловск. 1982. с. 9-21.

167. Стадухин В.Д., Иванов Н.А. Определение содержания железа в рудах месторождения г. Магнитной методом искусственного подмагничивания // Геофизические методы поисков и разведки рудных и нерудных месторождений. Межвузовский сборник. Свердловск. 1979. Вып. 5. с. 100-106.

168. Стадухин В.Д., Медведев А.Е., Туранов В.Н. Дифференциальное магнитное профилироввание в поле прямоугольных рамок // Разведочная геофизика. 1977. Вып. 76. с. 108114.

169. Стадухин В.Д., Туранов В.Н., Ундзенков Б.А. Совмещенное магнитное профилирование и зондирование на аномалиях Тургайского прогиба // Геофизические методы поисков и разведки. Свердловск. 1975. Вып. 1. с. 59-67.

170. Стадухин В.Д., Туранов В.Н., Шабанова Н.Н., Медведев А.Е. Захарченко В.Ф., Вершинин В.С. Метод искусственного подмагничивания при поисках магнетитовых месторождений. Методические рекомендации. Свердловск: УНЦ АН СССР. 1982. 108 с.

171. Стадухин В.Д., Улитина Г.Г. Магнитное профилирование квадратными и прямоугольными рамками на Теченском железорудном месторождении // Методические вопросы рудной геофизики Урала. Геофизический сборник. Свердловск. 1965. № 4. с. 73-77. 
(Труды Института геофизики УФ АН СССР. Вып. 3).

172. Стадухин В.Д., Улитина Г.Г. Общие приемы интерпретации результатов измерений при искусственном подмагничивании // Методика магнитных измерений и интерпретации в геофизике. Свердловск. 1970. с. 7-16. - (Труды Института геофизики УФ АН СССР. Вып. 5).

173. Стадухин В.Д., Шабанова Н.Н. К вопросу об определении природы магнитных аномалий по магнитным и электрическим свойствам возмущающих объектов // Электрометрические исследования при поисках и разведке рудных месторождений. Свердловск. 1977. с. 133-138.

174. Стадухин В.Д., Шабанова Н.Н., Шумаков Ю.В. Методика и результаты моделирования по методу искусственного подмагничивания // Геология и геофизика. 1975. № 1. c. $119-124$.

175. Стадухин В.Д., Шабанова Н.Н., Шумаков Ю.В. Методика интерпретации результатов магнитного зондирования // Разведочная геофизика. 1979. Вып. 85. с. 120-130.

176. Страхов В.Н. К теории двумерной задачи магниторазведки // Известия АН СССР. Серия геофизическая. 1959. № 2. с. 244-253.

177. Страхов В.Н. К теории плоской обратной задачи магнитного потенциала при переменной намагниченности // Известия АН СССР. Физика Земли. 1970. № 3. с. 44-58.

178. Страхов В.Н. К теории метода искусственного подмагничивания // Известия АН СССР. Физика Земли. 1977. № 7. с. 56-74.

179. Страхов В.Н. Математическое моделирование в теории интерпретации гравитационных полей // Математическое и физическое моделирование железорудных месторождений и рудных полей. Новосибирск: СНИИГГИМС. 1983. с. 7-17.

180. Страхов В.Н. Теоретические основы моделирования в грави- и магниторазведке // Вопросы теории и практики интерпретации гравитационных и магнитных аномалий при поисках и разведке месторождений полезных ископаемых. Иркутск. ВостСибСНИИГГиМС. 1987. c. 3-4.

181. Страхов В.Н., Блох Ю.И. Влияние размагничивания на определение гармонических моментов двухмерных тел произвольной формы // Разведочная геофизика. 1977. вып.75. с. 7681.

182. Страхов В.Н., Блох Ю.И. Аппроксимационный способ определения гармонических моментов двухмерных намагниченных объектов // Разведочная геофизика. 1977. вып.75. с. 8286.

183. Страхов В.Н., Лапина М.И. Определение интегральных характеристик возмущающих масс аппроксимационным методом в задачах гравиметрии и магнитометрии // Известия АН СССР. Физика Земли. 1975. № 4. с. 35-58.

184. Страхов В.Н., Лапина М.И., Ефимов А.Б. Решение прямых задач гравиметрии и магнитометрии на основе новых аналитических представлений для элементов полей от типовых аппроксимирующих тел. І. // Известия АН СССР. Физика Земли. 1986. № 6. с. 55-69.

185. Страхов В.Н., Лапина М.И., Ефимов А.Б. Решение прямых задач гравиметрии и магнитометрии на основе новых аналитических представлений для элементов полей от типовых аппроксимирующих тел. II. // Известия АН СССР. Физика Земли. 1986. № 7. с. 66-78.

186. Стрэттон Дж. А. Теория электромагнетизма. М-Л: ГИТТЛ. 1948. 539 с.

187. Тамм И.Е. Основы теории электричества. М.-Л.: Гостехиздат. 1949. 627 с.

188. Терехова Р.В. Использование суточных геомагнитных вариаций для определения природы магнитных аномалий // Геология, магнетизм горных пород и палеомагнетизм Южного Урала. Уфа: Институт геологии БФ АН СССР. 1977. с. 85-93.

189. Тимофеев А.Н. Об интерпретации магнитных аномалий в случае изменяющейся магнитной восприимчивости горных пород // Труды ИГГ СО АН СССР. 1960. № 1. с. 137-145.

190. Тозони О.В. Метод вторичных источников в электротехнике. М: Энергия. 1975. 296 c.

191. Тот Л.Ф. Расположения на плоскости, на сфере и в пространстве. М.: Главное издательство физико-математической литературы. 1958. 
192. Трухин В.И. Введение в магнетизм горных пород. М.: МГУ. 1973. 275 с.

193. Туранов В.Н. Опыт искусственного подмагничивания и изучение геомагнитных вариаций // Геолого-геофизические методы прогнозной оценки магнетитовых месторождений (на примерах Тургайского прогиба, Центрального и Восточного Казахстана). Алма-Ата. 1976. c. 37-40.

194. Туранов В.Н., Стадухин В.Д. Результаты применения метода искусственного подмагничивания в Центральном Казахстане // Теория и практика метода искусственного подмагничивания при поисках железорудных месторождений. Свердловск. 1982. с. 22-27.

195. Ундзенков Б.А. Магнитовариационный метод определения направления индукционной и остаточной намагниченности возмущающих тел в их естественном залегании // Магнетизм горных пород и палеомагнетизм. 1968 г. Сборник реферативных статей. М: ИФЗ АН CCCP. 1969. с. 200-203.

196. Ундзенков Б.А. Об эффективности магнитовариационного метода при поисках и разведке магнетитовых руд // Скарново-магнетитовые месторождения Урала. Свердловск: УНЦ АН СССР. 1978. с. 114-139.

197. Ундзенков Б.А., Борисова З.И. Экспериментальные данные о выделении влияния остаточной намагниченности на магнитные аномалии по измерениям трех компонент геомагнитных вариаций // Магнетизм горных пород и палеомагнетизм. 1968. Сборник реферативных статей. М: ИФЗ АН СССР. 1969. с. 204-207.

198. Филатов В.А. О единственности решения обратной задач в методе искусственного подмагничивания // Геология и геофизика. 1968. № 1 (97). с. 111-116.

199. Филатов В.А. О численном решении обратной задачи в методе искусственного подмагничивания // Геология и геофизика. 1968. № 6. с. 92-98.

200. Филатов В.А. Расчет магнитного поля от двухмерного тела произвольного сечения // Физика Земли. 1969. № 2. с. 82-91.

201. Филатов В.А., Филатова В.Н. Магнитные вариации в аномальном поле // Вопросы рудной геофизики Сибири. Труды СНИИГГИМСа. 1969. Вып. 92. с. 3-18.

202. Филатов В.А., Филатова В.Н., Воробьева Л.П. Приближенное вычисление вторичного поля в методе искусственного подмагничивания // Вопросы рудной геофизики Сибири. Новосибирск. 1967. с. 134-140. (Труды. СНИИГГИМС. Вып. 53).

203. Филатов В.В. Магнитное поле объемных зарядов // Геофизические методы поисков и разведки рудных и нерудных месторождений. Свердловск: СГИ. 1979. вып. 5. с. 46-52.

204. Филатов В.В. К методике моделирования магнитного воля железорудных месторождений Тагило-Кушвинского района // Геофизические методы поисков и разведки рудных и нерудных месторождений. Свердловск: СГИ. 1981. вып. 7. с. 38-44.

205. Фингер Д.Л. Влияние переменной нагрузки сжатия на магнитные свойства магнетита // Известия АН СССР. Серия геофизическая. 1951. № 5. с. 51-67.

206. Финкельштейн М.И. О влиянии электропроводности на магнитовариационный эффект // Земная кора Казахстана. Труды Института геологических наук АН КазССР. т. 30. Алма-Ата. 1971. с. 77-81.

207. Финкельштейн М.И. Некоторые критерии использования геомагнитных вариаций при определении природы магнитных аномалий // Известия АН КазССР. Серия геологическая. 1972. № 3. c. 39-44.

208. Финкельштейн М.И. Исследование возможности определения геологической природы объектов магнитных аномалий по магнитным вариациям. Автореферат кандидатской диссертации. Алма-Ата: КПИ. 1973.

209. Финкельштейн М.И., Гусманов Г.А. О вычислении нижней кромки намагниченных тел с использованием магнитных вариаций // Геофизические исследования при поисках и разведке рудных месторождений в Казахстане. Тезисы докладов Республиканской научнотехнической конференции по рудной геофизике. Алма-Ата. 1978. с. 62-63.

210. Финкельштейн М.И., Гусманов Г.А. Определение магнитных свойств железных руд месторождения Сарыбулак в их естественном залегании // Геофизические исследования при 
поисках и разведке рудных месторождений в Казахстане. Тезисы докладов Республиканской научно-технической конференции по рудной геофизике. Алма-Ата. 1978. с. 172-174.

211. Франк Ф., Мизес Р. Дифференциальные и интегральные уравнения математической физики. М.: ОНТИ. 1937. 998 с.

212. Франтов Г.С., Новожилов Г.Н., Савин А.Н. Индуктивное дипольное профилирование при поисках магнетитовых руд // Разведка и охрана недр. 1975. № 3. с. 41-45.

213. Хамидов С.X. К вопросу использования вариаций геомагнитного поля для определения остаточной намагниченности и магнитной восприимчивости магнетитовых руд на месторождениях Сюрената и Надыр // Аннотации научно-исследовательских работ химикотехнологического, геолого-разведочного и горно-металлургического факультетов. ТашПИ. Ташкент. 1964. с. 77-78.

214. Цирульский А.В. О единственности решения обратной задачи теории потенциала в методе искусственного подмагничивания // Известия АН СССР. Физика Земли. 1972. № 9. с. 6467.

215. Цирульский А.В. К теории метода искусственного подмагничивания // Известия АН СССР. Физика Земли. 1974. № 9. с. 70-77.

216. Цирульский А.В. Функции комплексного переменного в теории и методах потенциальных геофизических полей. Свердловск: УрО АН СССР. 1990. 133 с.

217. Цирульский А.В., Мартышко П.С. Об учете размагничивающего эффекта в задачах магниторазведки // Известия АН СССР. Физика Земли. 1979. № 3. с. 49-57.

218. Цирульский А.В., Мартышко П.С., Гуревич Ю.М. О возможности разбраковки магнитных аномалий по данным метода искусственного подмагничивания // Глубинное строение и полезные ископаемые востока СССР. Владивосток: Институт тектоники и геофизики. 1985. с. 54-69.

219. Чаро-Токкинская кремнисто-железорудная формация / Горелов Г.Ф., Гузман А.Г., Калугин И.А. и др. Новосибирск: Наука. 1984. 161 с.

220. Челишвили М.Л. Магнитное поле некоторых моделей геологических структур // Труды НИИ земного магнетизма АН СССР. 1955. вып. 11 (21).

221. Чудинова А.А. Интегральное уравнение обратной задачи электроразведки // Известия вузов. Математика. 1965. № 6. с. 1080-1088.

222. Шабанова Н.Н. Усовершенствованная методика интерпретации результатов магнитного зондирования // Теория и практика метода искусственного подмагничивания при поисках железорудных месторождений. Свердловск: Институт геофизики УНЦ АН СССР. 1982. c. $34-40$.

223. Шабанова Н.Н., Кузьмин А.Г. Изучение аномалий магнитного поля методом искусственного подмагничивания и оценка их перспективности по результатам работ ЧГРЭ в пределах Челябинской и Курганской областей за период с 1958 по 1989 гг. // Вопросы метода искусственного подмагничивания. Свердловск: Ин-т геофиз. УрО АН СССР. 1991. с. 2-56. (Деп. ВИНИТИ. № 3669-В91).

224. Шапиро В.А. Намагниченность массивной залежи магнетита // Методика магнитных намерений и интерпретации в геофизике. Свердловск: УФ АН СССР. 1970. с. 29-46.

225. Шапиро В.А., Иванов Н.А. Динамическая остаточная намагниченность и влияние ударов на остаточную намагниченность сильномагнитных горных пород // Доклады АН СССР. 1967. т. 173. № 5. с. 1065-1067.

226. Шапошникова Н.Ю. Аппроксимационнный метод оценки интегральных параметров железорудных месторождений скарново-магнетитового типа // Поиски и прогнозная оценка глубокозалегающих месторождений скарново-магнетитовых руд. М: ВИМС. 1977. с. 51-62.

227. Шевченко Н.Ф. К определению магнитных параметров руд по вариациям геомагнитного поля // Тезисы научно-исследовательских работ по геологоразведочному факультету Ташкентского политехнического института. Ташкент. 1966. с. 44-45.

228. Шевченко Н.Ф., Корякин А.П., Антонец А.Г. Использование метода вариаций при определении природы магнитных аномалий // Труды Ташкентского политехнического 
института. Ташкент. 1973. вып. 95. с. 43-44.

229. Шолпо Л.Е. Использование магнетизма горных пород для решения геологических задач. Л: Недра. 1977. 182 с.

230. Яковлев А.А. Аномальное поле включений в модели двухслойной среды при возбуждении сторонним потенциальным полем // Вопросы метода искусственного подмагничивания. Свердловск: Институт геофизики УрО АН СССР. 1991. с. 77-84. (Деп. ВИНИТИ. № 3669-В91).

231. Якубовский Ю.В. Индуктивные методы электроразведки. М: Госгеолтехиздат. 1963. $211 \mathrm{c}$.

232. Якубовский Ю.В., Ренард И.В. Применение метода вертикальных индукционных зондирований для изучения магнитных геоэлектрических разрезов // Известия вузов. Геология и разведка. 1974. № 11.

233. Яновский Б.М. О вариациях элементов земного магнетизма в аномальном поле // Труды ГГО. 1938. вып. 17. с. 77-91.

234. Яновский Б.М. Земной магнетизм. Т. 2. Теоретические основы магнитометрического метода исследования земной коры и геомагнитные измерения. Л: Изд-во ЛГУ. 1963. 461 с.

235. Яновский Б.М. Земной магнетизм. Л: Изд-во ЛГУ. 1978. 592 с.

236. Am K., Stemland R.O. Experimental determination of effective demagnetizing factors for a short cylinder // Geophysics. 1975. V. 40. No. 3. p. 527-529.

237. Courtillot V., Le Mouël J.-L. The study of Earth's magnetism (1269-1950): A foundation by Peregrinus and subsequent development of geomagnetism and paleomagnetism // Rev. Geophys. 2007. v. 45. RG3008, doi:10.1029/2006RG000198.

238. Debye P. Der Lichtdruck auf Kugeln von beliebigem Material // Annalen der Physik. Vierte Folge. 1909. Band 30. No. 1. p. 57-136.

239. Escola L. Numerical modeling of magnetic anomalies by means of the method of subareas // Report Univ. Oulu. Dept. Geophys. 1981. No. 1. p 15-20.

240. Escola L., Tervo T. Solving the magnetostatic field problem (a case of high susceptibility) by means of the method of subsectons // Geoexploration. 1980. v. 18. No. 2. p. 75-95.

241. Goldstein N.E., Ward S.H. The separation of remanent from induced magnetism in situ // Geophysics. 1966. v. 31. No. 4. p. 779-796.

242. Green G. Mathematical papers of George Green. NY: Chelses. 1970. 336 p.

243. Hjelt S.E., Avdevich M.M., Escola L., Tervo T. Interaction effects of magnetized bodies // Report Univ. Oulu. Dept. Geophys. 1981. No. 4. p. 41-50.

244. Hvoždara M. Solution of the direct problem of magnetometry with the aid of the potential of dipole layer // Contr. Geophys. Inst. Slov. Acad. Sci. 1983. v. 14. p. 23-46.

245. Joseph R.T., Shlomann E. Demagnetizing field in nonellipsoidal bodies // Journal of applied physics. 1965. v. 36. No. 5.

246. Kostrov N.P. Calculation of magnetic anomalies by 2D bodies of arbitrary shape with consideration of demagnetization // Geophysical prospecting. 2007. v. 55. p. 91-115.

247. Lee T.J. Rapid computation of magnetic anomalies with demagnetization included, for arbitrary shaped magnetic bodies // Geophys. Journal of the Royal Astron. Soc. 1980. v. 60. No. 1. p. 67-75.

248. Mauersberger P. Ein iterationsverfahren zur berechnung des indusierten magnetostatishen Storefeldes eines beliebig gestalteten Storkorpers // Gerlands beitrage zur geophysik. 1966. v. 75. No. 4. p. 497-509.

249. Mie G. Beiträge zur Optik trüber Medien, speziell kolloidaler Metallösungen // Annalen der Physik. Vierte Folge. 1908. Band 25. No. 3. p. 377-445.

250. Nagata T., Kinoshito H. Effect of release of compression on magnetization of rocks and assembles of magnetic minerals // Nature. 1964. v. 204 (4964). p. 1183-1184.

251. Sharma P.V. Rapid computation of magnetic anomalies and demagnetization effects caused by bodies of arbitrary shape // Pure and applied geophysics. 1966. v. 64. No. 2. p. 89-109.

252. Sharma P.V. Demagnetization effect of a rectangular prism // Geophysics. 1968. v. 33. 
No. 1. p. 132-134.

253. Stacey F.D. Effect of stress on the remanent magnetism of magnetite-bearing rocks // Journal of Geophysical Research. 1958. v. 63. No. 2. p. 361-368.

254. Stacey F.D. Effect of release of compression on magnetization of rocks and assembles of magnetic minerals // Nature. 1964. v. 204 (4964). p. 1184-1185.

255. Thalén R. Sur la recherche des mines de fer a l'aide des mesures magnétiques // Nova acta Regiae societatis scientiarum. Uppsala. Ser III. 1877. 36 р. (Перевод: Тален Р. Исследование местностей на месторождения железных руд посредством магнитных измерений // Горный журнал. 1883. т. 1. № 2. с. 179-264).

256. Vogel A. Demagnetizing fields of inhomogeneous induced magnetization // Nature. 1962. v. 196. No. 4861.

257. Vogel A. The application of electronic computers to the calculation of effective magnetization // Geophysical prospecting. 1963. v. 11. N 1. p. 51-58.

258. Vogel A. Genauigkeit und konvergenz eines iterativen verfahrens zur berechnung der effektiven induktionsmagnetisierung von modellen // Gerlands beitrage zur geophysik. 1963. v. 72. No. 4. p. 333-341.

259. Wait J.R. A conducting sphere in a time varying magnetic field // Geophysics. 1951. v. 16. No. 4. p. 666-672.

260. Ward S.H. Unique determination of conductivity, susceptibility, size and depth in multifrequency electromagnetic exploration // Geophysics. 1959. v. 24. No. 3. p. 531-546.

261. Ward S.H. The electromagnetic response of magnetic iron ore deposit // Geophysical prospecting. 1961. v. 9. No. 2. p. 191-202.

262. Weigand A. Die anganaberte berechnung ebener und rotationssymmetrisher potentialfelder mit hifte des differensenverfahrens. Technik. Berlin. 1953.

263. Zidarov D.P. Sur l'aimentation inductive des corps magnetiques pessedant une aimantation permanente // Доклады Болгарской АН. 1966. № 9. с. 783-786.

264. Zidarov D.P. Numerical determination of magnetization of magnetically soft bodies // Доклады Болгарской АН. 1974. т. 27. № 3.

265. Zietz I., Henderson R.G. A preliminary report on model studies of magnetic anomalies of three-dimensional bodies // Geophysics. 1956. v. 21. No. 3. p. 794-814.

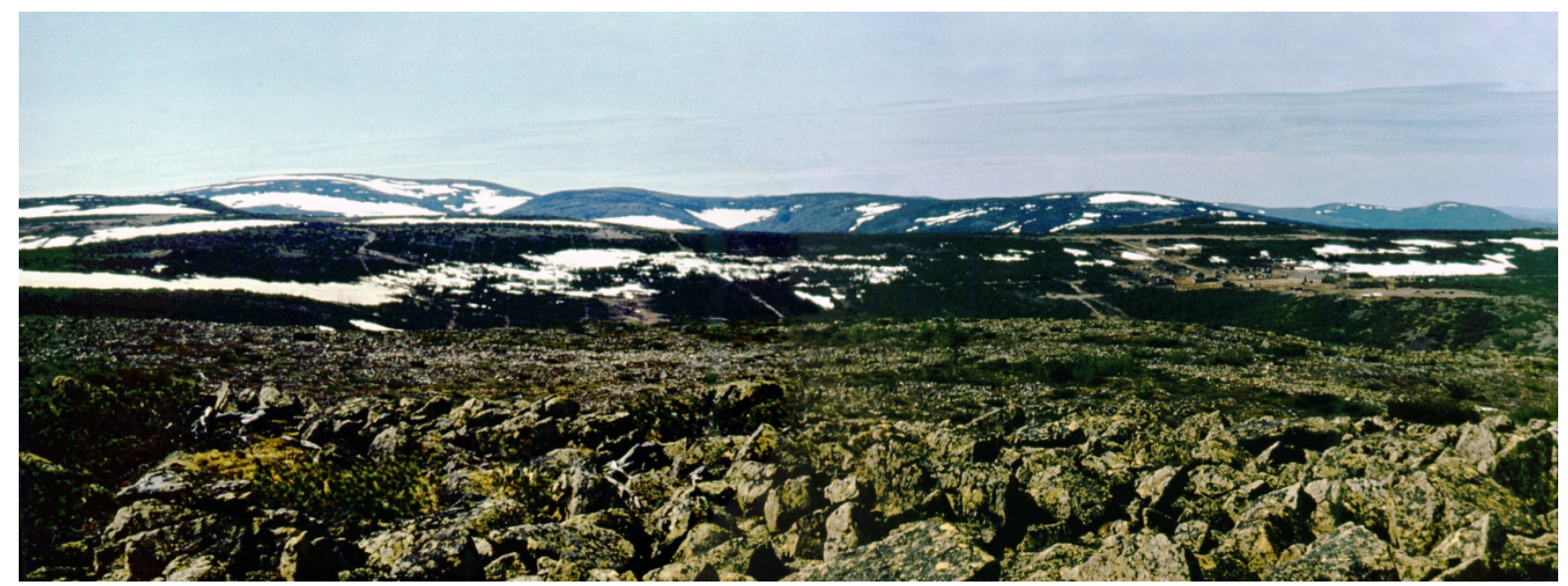

Месторождение Тарыннах (Южная Якутия) в период разведки. Фото автора, 1983 г. 